\title{
FOURIER AND VARIATIONAL BASED APPROACHES FOR FINGERPRINT SEGMENTATION
}

\author{
Dissertation \\ zur Erlangung des mathematisch-naturwissenschaftlichen \\ Doktorgrades \\ "Doctor rerum naturalium" \\ der Georg-August-Universität Göttingen
}

vorgelegt von

Duy Hoang Thai

aus Göttingen

Göttingen 2015 
Referent: Professor Dr. Axel Munk, Institut für Mathematische Stochastik, Universität Göttingen

Korreferent: Professor Dr. Preda Mihăilescu, Mathematische Institut, Universität Göttingen

Tag der mündlichen Prüfung: 28.01.2015 


\section{Abstract}

Fingerprint recognition plays an important role in many commercial applications and is used by millions of people every day, e.g. for unlocking mobile phones. Fingerprint image segmentation is typically the first processing step of most fingerprint algorithms and it divides an image into foreground, the region of interest (ROI), and background. Two types of error can occur during this step and both have a negative impact on the recognition performance: 'true' foreground can be labelled as background and features like minutiae can be lost, or conversely 'true' background can be misclassified as foreground and spurious features can be introduced.

The contribution of this study is threefold:

1. We propose the feature extractors for fingerprint images with Fourier and variational based approaches (see Chapter 2 and Chapter 3).

In the Fourier domain (cf. [1]), we assume that fingerprint patterns mostly stay in a specific range of frequencies. We introduce a novel factorized directional bandpass (FDB) segmentation method based on the directional Hilbert transform of a Butterworth bandpass (DHBB) filter interwoven with soft-thresholding for feature extraction. Then, a morphological operator is applied on these extracted features to obtain the ROI.

In the variational approach (cf. [2]), fingerprint images are characterized by a smooth, curved and oriented pattern which has a sparse representation in certain transform domains. Based on this assumption, we propose a model for global three-part decomposition (G3PD) which takes the nature of the texture occurring in real fingerprint images into account. The G3PD method decomposes the original fingerprint image into a piecewise constant image, "texture image" and noise image. The decomposition is obtained by finding the minimizer of the convex minimization $\mathrm{TV}-G_{1,1}^{3 / 4}-\ell_{1}-G_{\infty, \infty}^{-3 / 4}$. After texture extraction by the G3PD method, the ROI is attained by morphological operators.

2. We provide a manually marked ground truth segmentation for 10560 images of the FVC database as an evaluation benchmark which is made publicly available.

3. We conduct a systematic performance comparison between our proposed methods and four of the most often cited fingerprint segmentation algorithms. The evaluation shows that our approaches clearly outperform these four widely used methods, especially the $\mathrm{TV}-G_{1,1}^{3 / 4}-\ell_{1}-G_{\infty, \infty}^{-3 / 4}$ model.

Keywords: Fingerprint recognition, fingerprint image segmentation, evaluation benchmark, manually marked ground truth, directional Hilbert transform, Riesz transform, Butterworth bandpass filter, texture extraction, Besov space, Curvelet decomposition space, variational method, convex minimization, functional analysis 


\section{Zusammenfassung}

Fingerabdruckerkennung spielt in vielen kommerziellen Anwendungen eine wichtige Rolle und wird von Millionen von Menschen täglich verwendet, z.B. für das Entsperren von Handys. Die Segmentierung von Fingerabdrücken ist typischerweise der erste Schritt der meisten Fingerabdruck-Algorithmen und teilt die Abbildung in den Vordergrund, den interessierenden Bereich (ROI) und den Hintergrund auf. Zwei Arten von Fehlern können bei diesem ersten Schritt auftreten, welche sich beide negativ auf das Verfahren auswirken: "Wahrer" Vordergrund kann als Hintergrund gekennzeichnet werden und Merkmale wie Minutien können verloren gehen oder umgekehrt kann "wahrer" Hintergrund fälschlicherweise als Vordergrund klassifiziert und störende Merkmale eingeführt werden.

Diese Arbeit leistet in dreifacher Weise einen Beitrag zum Lösen dieser Probleme:

1. Wir behandeln die Merkmalextraktion für Fingerabdrücke mit den auf Fouriertransformation und Variationsrechnung basierenden Ansätzen, welche in Kapitel 2 bzw. Kapitel 3 beschrieben werden. Wir nehmen an, dass Fingerabdruckmuster meistens in einem bestimmten Frequenzbereich bleiben. Deshalb führen wir ein neuartiges "factorized directional bandpass" (FDB) Segmentierungsverfahren basierend auf einer Richtung folgenden Hilberttransformation eines ButterworthBandpasses (DHBB) verbunden mit weichem Thresholding zur Merkmalsextraktion ein. Dann wird der morphologische Operator auf diese extrahierte Merkmale angewendet, um den ROI zu erhalten.

Für den auf Variationsrechnung basierenden Ansatz werden Fingerabdrücke durch glatte, kurvige und orientierte Muster, die eine dünn-besetzte Darstellung bezüglich bestimmter Transformationen haben. Basierend auf dieser Annahme behandeln wir ein Modell zur globalen "three-part decomposition" (G3PD), welche die Natur der Textur berücksichtigt, die im Fingerabdruck vorkommt. Das G3PD-Verfahren zerlegt den Fingerabdruck in die stückweise konstante Abbildung, Textur und Rauschen. Die Zerlegung erhält man durch das Finden des Minimierers für das konvexe Minimierungsproblem TV $-G_{1,1}^{3 / 4}-\ell_{1}-G_{\infty, \infty}^{-3 / 4}$. Nach der Extraktion der Textur durch das G3PD-Verfahren erhält man die ROI durch den morphologischen Operator.

2. Wir liefern eine manuell markierte Segmentierung für 10560 Abbildungen in der FVC-Datenbank als Bewertungsmaßstab, der öffentlich zugänglich gemacht wurde.

3. Wir implementieren einen systematischen Leistungsvergleich zwischen unserem vorgeschlagenen Verfahren und vier der meistzitierten Fingerabdrucksegmentierungsalgorithmen. Der Vergleich zeigt, dass unsere Methode diese weitverbreiteten Verfahren deutlich übertrifft, besonders im TV $-G_{1,1}^{3 / 4}-\ell_{1}-G_{\infty, \infty}^{-3 / 4}$-Modell. 
Stichworte: Fingerabdruckerkennung, Fingerabdruck-Bildsegmentierung, Benchmark, manuell markierte Segmentierung, Richtungen folgende Hilbert-Transformationen, Riesz-Transformationen, Butterworth-Bandpassfilter, Textur-Extraktion, Besov-Raum, Curvelet Zersetzungsraum, auf Variationsrechnung basierende Verfahren, konvexe Minimierung, Funktionalanalysis 


\section{Dedication}

"To my family for all sacrifices they have made over the years for my education" 


\section{Acknowledgements}

This study demonstrates the primary part of my scientific research as a research assistant at the Institute for Mathematical Stochastics in Goettingen during around three years. With the background in electronics, I set a very first step in image processing by finalizing the bachelor degree with the thesis: "Computer vision in controlling the movement of robot". Later, the adage "A picture is worth a thousand words" has created my passion for image analysis, especially medical images, bio-imaging and biometrics. Thus, it pushed me to graduate the master degree in Robotics and Vision Lab in Korea with the dissertation "Human iris recognition". Based on the engineering background in control theory and image analysis, in order to make a deeper understanding, I found that applying the mathematical model to describe the problem is the fundamental. Fortunately, I got a position in the Institute for Mathematical Stochastics in Goettingen where I have had a chance to work with mathematicians who help me to discover the beauty of maths. With the application in fingerprint image analysis, I have applied mathematical models to reconstruct good features from the noisy data with different illuminations such that it satisfies some constraints, e.g. a certain of smoothness and sparseness.

I would like to express my thankfulness to the people who influenced this work, especially my supervisor Prof. Axel Munk. He gave me a chance to work on a very interesting topic and taught me to become an independent researcher, and scientifically guided during my study, especially for his contribution in Chapter 3.

Moreover, I am grateful to Prof. Preda Mihăilescu for assuring the quality of my work.

I would like to thank to Dr. Carsten Gottschlich, for his valuable recommendations and for sharing his expertise in computer science view.

I would also like to give a big thank to Prof. Stephan Huckemann for his kindness in cooperation on Chapter 2, comments on Chapter 3 and for helping me to see things in a positive way in mathematics.

Big thank to Prof. Tatyana Krivobokova, Dr. Florian Völlering, Dr. Klaus Frick, Dr. Johannes W.R. Martini, Fabian Telschow, Qinghai Guo, Housen Li and Fabian Kück, especially to Dr. Benjamin Eltzner, for their technical advisors in mathematics.

I thank to Jun.-Prof. Andrea Krajina for her kindness responses in the extreme value theory in Chapter 3 and comments on the text.

I would like to thank Prof. Gerlind Plonka-Hoch, Prof. David Russell Luke, Dr. Yvo Pokern for their careful reading and valuable comments on Chapter 2 and Chapter 3 which mainly form my thesis.

I am grateful to the members of my committee - Prof. Axel Munk, Prof. Preda 
Mihăilescu, Prof. Stephan Huckemann, Prof. Gerlind Plonka-Hoch, Prof. Jens Grabowski and Prof. Stephan Waack - for accepting to read and comment on my thesis.

I thank to members in the Institute for Mathematical Stochastics for their assistance and cooperation. Special thanks to my officemate, Dr. Benjamin Tams, for sharing his ideas and assisting to overcome the hard times during my stay in Goettingen.

Of course, this work would be impossible without my dear family, in particular my parents who have created the inspiration for my scientific career and my sister for the strong support during these years.

Finally, I would like to send special thanks to my friends in Goettingen for the wonderful moments that we have passed together.

Duy Hoang Thai

\section{Goettingen}

October 2014 



\section{Contents}

Abstract

Zusammenfassung iv

Acknowledgements vii

1 Introduction 1

1.1 Feature for Fingerprint Segmentation . . . . . . . . . . . . . . . . 1

1.2 The Fourier and Variational Based Approaches . . . . . . . . . . . . 1

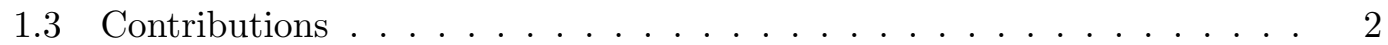

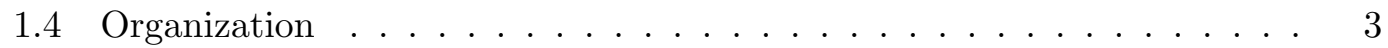

2 Filter Design and Performance Evaluation for Fingerprint Image Segmenta$\begin{array}{ll}\text { tion } & 4\end{array}$

2.1 Introduction . . . . . . . . . . . . . . . . . . 4

2.2 Fingerprint Segmentation by FDB Methods . . . . . . . . . . . . 6

2.3 Evaluation Benchmark and Results . . . . . . . . . . . . . . . . . 14

2.4 Conclusions . . . . . . . . . . . . . . . . . . . . . . . . . . 18

Supplementary Appendix . . . . . . . . . . . . . . . . . . . . . . 19

3 Global Variational Method for Fingerprint Segmentation By Three-Part De$\begin{array}{ll}\text { composition } & 34\end{array}$

3.1 Introduction . . . . . . . . . . . . . . . . . . . . . 34

3.2 The G3PD Method for Fingerprint Segmentation . . . . . . . . . . . 37

3.3 Evaluation: Benchmark, Protocol and Experimental Results . . . . . . . 50

3.4 Conclusions . . . . . . . . . . . . . . . . . . . . . 53

Supplementary Appendix . . . . . . . . . . . . . . . . 68

$\begin{array}{llr}4 & \text { Conclusion } & 94\end{array}$

4.1 Main Contributions . . . . . . . . . . . . . . . . . . . . 94

4.2 Future Work . . . . . . . . . . . . . . . . . . . 95

$\begin{array}{ll}\text { Bibliography } & 96\end{array}$

$\begin{array}{ll}\text { Curriculum Vitae } & 103\end{array}$ 


\section{Introduction}

\subsection{Feature for Fingerprint Segmentation}

Nowadays, fingerprint recognition is used by millions of people in their daily life for verifying a claimed identity in commercial applications ranging from check-in at work places or libraries, access control at amusement parks or zoos to unlocking notebooks, tablets or mobile phones. Most fingerprint recognition systems are based on minutiae as features for comparing fingerprints [3]. Typical processing steps prior to minutiae extraction are fingerprint segmentation, orientation field estimation and image enhancement. The segmentation step divides an image into foreground, the region of interest (ROI), and background. Two types of error can occur in this step and both have a negative impact on the recognition rate: 'true' foreground can be labelled as background and features like minutiae can be lost, or 'true' background can be misclassified as foreground and spurious features may be introduced. It is desirable to have methods that control both errors.

The question is: what is the important feature of the fingerprint pattern to distinguish with the non-fingerprint pattern, e.g. the homogeneous regions or the small scale objects (noise), etc? The answer is: fingerprint image contains the repeated patterns, called texture, which stays in a specific frequency range in the Fourier domain, called frequencies occurring in true fingerprint images (FOTIs). The texture satisfies a certain level of smoothness and sparsity in the spatial domain. Therefore, we consider the fingerprint feature in two views:

1. In the Fourier domain, fingerprint pattern is considered as a smooth texture whose frequency spectrum mostly stays in the FOTIs.

2. In the spatial domain, fingerprint images are characterized by a smooth, curved and oriented pattern which has a sparse representation in certain transform domains.

In the next section, we discuss some methods to adapt these ideas for fingerprint feature extraction. Then, the ROI is obtained by the morphological operator.

\subsection{The Fourier and Variational Based Approaches}

Firstly, we consider the feature extraction for the fingerprint image in the Fourier domain. As mentioned before, the spectrum of fingerprint pattern almost stays in the FOTIs, so we design the factorized directional bandpass (FDB) method to capture the pattern in this FOTIs. Since the signal is known to be sparse in some transform domains, we propose a novel FDB segmentation method based on the directional Hilbert 
transform of a Butterworth bandpass (DHBB) filter to obtain the features in the different directional subbands. Due to the smoothness of fingerprint images, the classical shrinkage operator is introduced in between to remove the small coefficients in these subbands which correspond to non-prominent FOTI features, including: the small scale objects (noise) and all FOTI features not due to the true fingerprint patterns. Finally, the ROI is attained by applying morphological operators on the true fingerprint feature in the FOTIs.

Secondly, in different view than the FDB method, the fingerprint pattern is represented as a sparse, smooth and oriented texture in the spatial domain. Thus, the feature of fingerprint image contains only texture without homogeneous regions and noise. Inspired by this idea, we propose a model for global three-part decomposition (G3PD) to decompose the original image into the homogeneous regions (or cartoon), texture and noise. The core of the G3PD is to find the solution of the convex minimization, i.e. TV $-G_{1,1}^{3 / 4}-\ell_{1}-G_{\infty, \infty}^{-3 / 4}$. The piecewise constant regions are measured by total variation (TV) norm. The sparsity and smoothness of texture can benefit from $\ell_{1}$ and $G_{1,1}^{3 / 4}$ norms, respectively. The dual space of $G_{1,1}^{3 / 4}$ is $G_{\infty, \infty}^{-3 / 4}$ which relates to the small curvelet coefficients. The space $G_{\infty, \infty}^{-3 / 4}$ is very suitable to capture the noise. By experiment, we have found that the curvelet shrinkage captures the geometry of fingerprint pattern better than the classical wavelet thresholding in terms of over-estimation and smoothness.

\subsection{Contributions}

In this thesis, we adopt the Fourier and variational based approaches to extract feature for fingerprint segmentation. The contribution of this study is mainly threefolds:

\section{The methods for fingerprint segmentation:}

- The factorized directional bandpass (FDB) method (see [1]):

Since the frequency spectrum of fingerprint pattern mostly stays in the FOTIs, we design the FDB method to capture this FOTIs. A novel FDB segmentation method is based on the directional Hilbert transform of a Butterworth bandpass (DHBB) filter interwoven with soft thresholding for texture extraction. Then, the morphological operator is applied on this texture to obtain the ROI.

- The global three-part decomposition (G3PD) method (see [2]):

We consider fingerprint pattern as a smooth, curved and oriented pattern which is sparse in some transform domains. In order to deal with this concept, we proposed the G3PD method based on the variational model, TV $-G_{1,1}^{3 / 4}-\ell_{1}-G_{\infty, \infty}^{-3 / 4}$, for the decomposition. Loosely speaking, the total variation (TV) norm measures the homogeneity of the piecewise-smooth regions. The $\ell_{1}$ norm and the $G_{1,1}^{3 / 4}$ norm simultaneously measure the sparsity and the smoothness of texture, respectively. The $G_{\infty, \infty}^{-3 / 4}$ measures the degree of the oscillation signal, i.e. noise. By solving the convex minimization $\mathrm{TV}-G_{1,1}^{3 / 4}-\ell_{1}-G_{\infty, \infty}^{-3 / 4}$, the smooth and sparse texture is obtained. Then, the morphological operator is also implemented to get the ROI. 
Benchmark: We provide a manually marked ground truth segmentation for all 12 databases of FVC2000 [4], FVC2002 [5] and FVC2004 [6]. Each databases consists of 80 images for training and 800 images for testing. Overall this benchmark consists of 10560 marked segmentation images. This ground truth benchmark is made publicly available, so that other researchers can evaluate segmentation algorithms on it.

Evaluation against existing methods: In order to clarify the feasibility of our approaches, we conduct a systematic performance comparison with widely used segmentation algorithms on this benchmark. In total, more than 100 methods for fingerprint segmentation can be found in the literature. However, it remains unclear how these methods compare with each other in terms of segmentation performance and which methods can be considered as state-of-the-art. In order to remedy the current situation we chose four of the most often cited fingerprint segmentation methods and compared their performance: a method based on mean and variance of gray level intensities and the coherence of gradients as features and a neural network as a classifier [7], a method using Gabor filter bank responses [8], a Harris corner response based method [9] and an approach using local Fourier analysis [10].

\subsection{Organization}

The cornerstone construction of the thesis - the Fourier and variational based approaches for fingerprint segmentation - are presented in Chapter 2 and Chapter 3, respectively. In particular, the FDB method with the DHBB filter in the Fourier domain is introduced to extract the feature for the morphology operator to obtain the ROI in Chapter 2. The fingerprint feature is considered as a sparse texture with a certain smoothness which is decomposed by the convex minimization in Chapter 3 by the G3PD model. The smoothness and sparsity correspond to $\ell_{1}$ norm of curvelet coefficient and $\ell_{1}$ norm of texture, respectively. We refer the reader to Chapter 3 for a deep discussion about the smoothness and the sparsity of the texture. We finalize the thesis with conclusion and an outlook to possible future works in Chapter 4. 


\section{Filter Design and Performance Evaluation for Fingerprint Image Segmentation}

\subsection{Introduction}

As mentioned before, segmentation is the first step in the fingerprint recognition. In this procedure, there are two types of error that impact on the recognition performance. In order to controls both errors, a Fourier based method for fingerprint segmentation is designed.

\subsubsection{The Factorized Directional Bandpass Method, Benchmark and Evaluation}

In order to balance both errors we take the viewpoint that - loosely speaking - fingerprint images are highly determined by patterns that have frequencies only in a specific band of the Fourier spectrum (prior knowledge). Focusing on these frequencies occurring in true fingerprint images (FOTIs), we aim at the following goals:

1) Equally preserving all FOTIs while attenuating all non-FOTIs.

2) Removing all image artifacts in the FOTI spectrum, not due to the true fingerprint pattern.

3) Returning a (smooth) texture image containing only FOTI features from the true fingerprint pattern.

4) Morphological methods returning the ROI.

In order to meet these goals we have developed a factorized directional bandpass (FDB) segmentation method.

The FDB method: At the core of the FDB method is a classical Butterworth bandpass filter which guarantees Goal 1. Notably Goal 1 cannot fully be met by Gaussian based filtering methods such as the Gabor filter. Obviously, due to the Gaussian bell shaped curve, FOTIs would not be filtered alike. Because straightforward Fourier methods cannot cope with curvature (as could e.g. curved Gabor filters [11]) we perform separate filtering into a few isolated orientations only, via directional Hilbert transformations. The composite directional Hilbert Butterworth bandpass filter (DHBB) incorporates our prior knowledge about the range of possible values of ridge frequencies (between $1 / 3$ and $1 / 25$ pixels) or interridge distances (between 3 and 25 pixels) [11], assuming a sensor resolution of 500 DPI and that adult fingerprints are processed. In the case of adolescent 
fingerprints [12] or sensors with a different resolution, the images can be resized to achieve an age and sensor independent size - not only for the first segmentation step, but also for all later processing stages. Our parameters can be tuned to reach an optimal tradeoff between treating all realistic frequencies alike and avoiding Gibbs effects. Moreover we use a data friendly rectangular spectral shape of the bandpass filter employed which preserves the rectangular shape of the spatial image.

A second key ingredient is the factorization of the filter into two factors in the spectral domain, between which a thresholding operation is inserted. After preserving all FOTIs and removing all non-FOTIs in application of the first factor, all FOTI features not due to the true fingerprint pattern (which are usually less pronounced) are removed via a shrinkage operator: soft-thresholding. Note that albeit removing less pronounced FOTI features, thresholding introduces new unwanted high frequencies. These are removed, however, by application of the second factor, which also compensates for a possible phase shift due to the first factor, thus producing a smoothed image with pronounced FOTI features only.

At this stage, non-prominent FOTI features have been removed, not only outside the ROI, but also some due to true fingerprint features inside the ROI. In the final step, these "lost" regions are restored via morphological operations (convex hull after binarization and two-scale opening and closing).

The careful combination of the above ingredients in our proposed FDB method yields segmentation results far superior to existing segmentation methods.

Benchmark: In order to verify this claim, because of the lack of a suitable benchmark in the literature, we contribute a manually marked ground truth segmentation for all 12 databases of FVC2000 [4], FVC2002 [5] and FVC2004 [6], totally 10560 marked segmentation images.

Evaluation: Based on the ground truth benchmark, we compare the performance of our proposed FDB method with four of the most often cited fingerprint segmentation methods in the literature, cf. [7], [8], [9] and [10].

\subsubsection{Related Work}

Early methods for fingerprint segmentation include Mehtre et al. [13] who segment an image based on histograms of local ridge orientation and in [14] additionally the gray-level variance is considered. A method proposed by Bazen and Gerez [7] uses the local mean and variance of gray-level intensities and the coherence of gradients as features and a neural network as a classifier. Similarly Chen et al. [15] use block based features including the mean and variance in combination with a linear classifier. Both methods perform morphology operations for postprocessing. A method by Shen et al. is based on Gabor filter bank responses of blocks [8]. In [11], all pixels are regarded as foreground for which a valid ridge frequency based on curved regions can be estimated. Wu et al. [9] proposed a Harris corner response based method and they apply Gabor responses for postprocessing. Wang et al. [16] proposed to use GaussianHermite moments for fingerprint segmentation. The method of Zhu et al. [17] uses a 
gradient based orientation estimation as the main feature, and a neural network detects wrongly estimated orientation and classifies the corresponding blocks as background. Chikkerur et al. [10] applied local Fourier analysis for fingerprint image enhancement. The method performs implicitly fingerprint segmentation, orientation field and ridge frequency estimation. Further approaches for fingerprint enhancement in the Fourier domain include Sherlock et al. [18], Sutthiwichaiporn and Areekul [19] and Bartůněk et al. $[20,21,22]$. Recently, segmentation methods for latent fingerprints were proposed, see Zhang et al. [23], Cao et al. [24], and the references therein. It would be of interest to see how these methods aiming at latent fingermarks perform on a benchmark of plain fingerprint images.

\subsubsection{Setup of Chapter 2}

This chapter is organized as follows: in the next section, we describe the proposed method beginning with the design of the DHBB filter for texture extraction in Section 2.2.1. Subsequently, the extracted and denoised texture is utilized for estimating the segmentation as described in 2.2.2 which summarizes the FDB segmentation procedure. In Section 2.3, the manually marked ground truth benchmark is introduced and applied for evaluating the segmentation performance of four widely used algorithms and for comparing them to the proposed FDB segmentation method. The results are discussed in Section 2.4.

\subsection{Fingerprint Segmentation by FDB Methods}

Our segmentation method uses a filter transforming an input 2D image $f(\cdot) \in L_{2}\left(\mathbb{R}^{2}\right)$ into a feature image

$$
\tilde{f}(\boldsymbol{x}):=\sum_{l=0}^{L-1} \sum_{\boldsymbol{x} \neq \boldsymbol{m} \in \mathbb{Z}^{2}} \underbrace{\mathbf{T}\{\underbrace{\left\langle f(\cdot), \phi_{l}^{\gamma, n}(\cdot-\boldsymbol{m})\right\rangle_{L_{2}}^{p v}}_{c_{l}[\boldsymbol{m}]}, \beta\}}_{d_{l}[\boldsymbol{m}]} \cdot \phi_{l}^{\gamma, n}(\boldsymbol{x}-\boldsymbol{m}) .
$$

Due to our filter design, the $L_{2}$ product above as well as all convolutions, integrals and sums are understood in the principal value sense

$$
\lim _{\epsilon \rightarrow 0} \int_{\|\boldsymbol{y}-\boldsymbol{m}\| \geq \epsilon} f(\boldsymbol{y}) \cdot \phi_{l}^{\gamma, n}(\boldsymbol{y}-\boldsymbol{m}) d \boldsymbol{y}=\left\langle f(\cdot), \phi_{l}^{\gamma, n}(\cdot-\boldsymbol{m})\right\rangle_{L_{2}}^{p v}=\left(f *_{p v} \phi_{l}^{\gamma, n, \vee}\right)(\boldsymbol{m}) .
$$

Having clarified this, the symbol "pv" will be dropped in the following. At the core of (2.1) is the DHBB filter conveyed by $\phi_{l}^{\gamma, n}$ ( $l$ counts directions, $n$ and $\gamma$ are tuning parameters providing sharpness). In fact, we suitably factorize the filter conveyed by $\phi_{l}^{\gamma, n} * \phi_{l}^{\gamma, n, \vee}$ in the Fourier domain where $\phi_{l}^{\gamma, n, \vee}(\boldsymbol{x}):=\phi_{l}^{\gamma, n}(-\boldsymbol{x})$ with the argument reversion operator " $\vee$ " and apply a thresholding procedure $\mathbf{T}$ "in the middle". Underlying this factorization is a factorization of the bandpass filter involved. The precise filter design will be detailed in the following. Note that the directional Hilbert transform is also conveyed by a non-symmetric kernel. Reversing this transform (as well as the factor of the Butterworth) restores symmetry. It is inspired by the steerable wavelet 


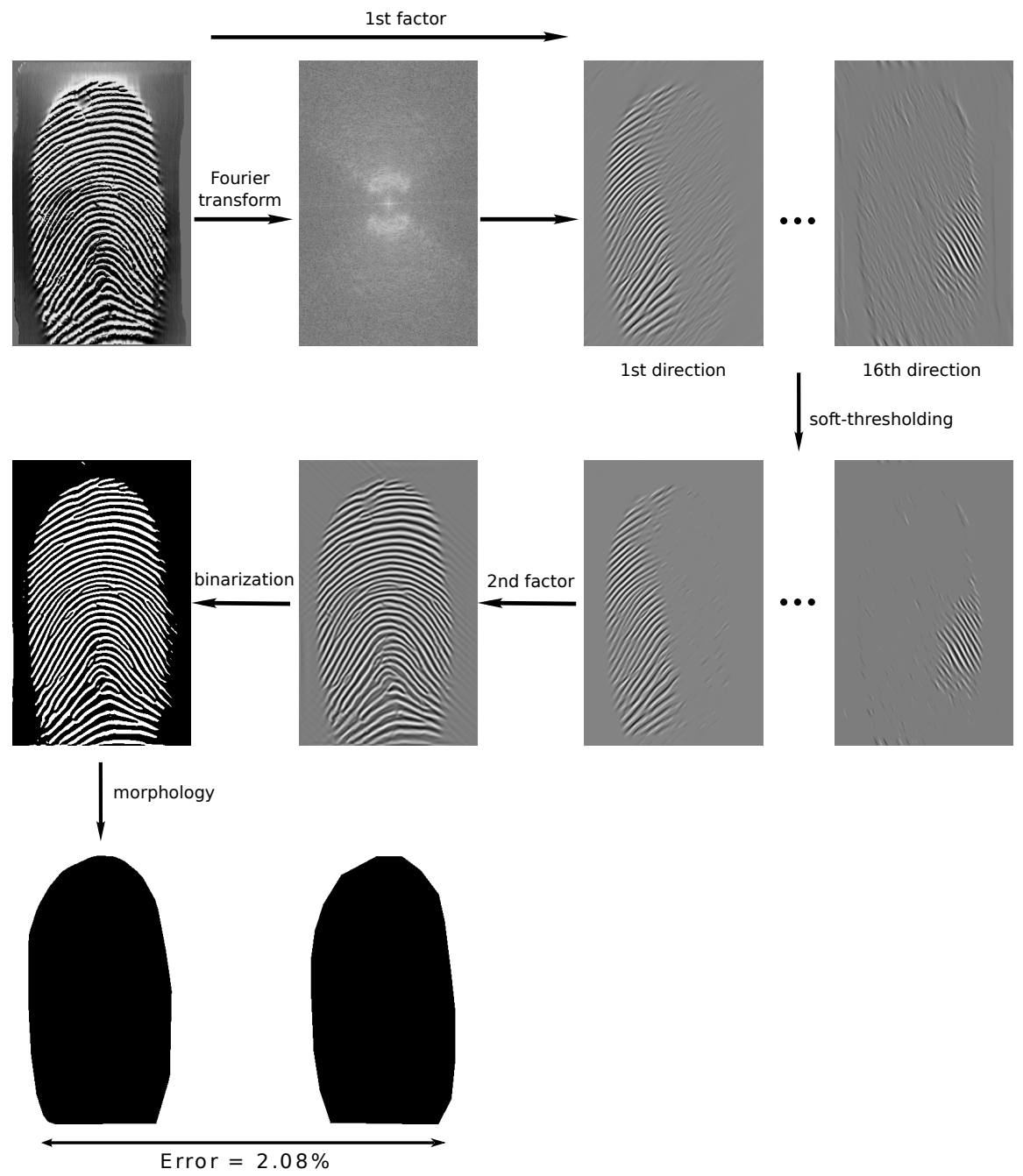

Figure 2.1: Overview over the segmentation by the FDB method: In the analysis step, the original image (top row, left) is transformed into the Fourier domain (second column) and filtered by the first DHBB factor obtaining 16 directional subbands (third and fourth columns). Next soft-thresholding is applied to remove spurious patterns (second row, third and fourth columns). In the synthesis step, the feature image (second column) is reconstructed from these subbands using the second DHBB factor. Finally, the feature image is binarized and the ROI is obtained by morphological operations. The estimated ROI (third row, left) is compared to manually marked ground truth segmentation (third row, right) in order to evaluate the segmentation performance. 
$[25,26,27]$ and to some extend similar in spirit to the curvelet transform [28], [29] and the curved Gabor filters [11]. We deal with curvature by analyzing single directions $l$ separately before the final synthesis.

Via factorization, possible phase shifts are compensated and unwanted frequencies introduced by the thresholding operator are eliminated, yielding a sparse smoothed feature image. This allows for easy binarization and segmentation via subsequent morphological methods, leading to the ROI.

Note that (2.1) can be viewed as an analog to the projection operator in sampling theory with the analysis and synthesis steps (e.g. [30]). In this vein we have the following three steps:

Forward analysis (prediction): A first application of the argument reversed DHBB filter to a fingerprint image $f$ corresponds to a number of directional selections in certain frequency bands of the fingerprint image giving $c_{l}[\boldsymbol{m}]$ above.

Proximity operator (thresholding): In order to remove intermediate coefficients due to spurious patterns (cf. [31]) we perform soft thresholding on the filtered grey values yielding $d_{l}[\boldsymbol{m}]$ above.

Backward synthesis: Subsequently we apply the filter (non-reversed) again giving $\tilde{f}$ assembled from all subbands. A numerical comparison to other synthesis methods, summation (corresponding to a naive reconstruction) and maximal response in the appendix 2.5, shows the superiority of this smoothing step.

Due to the discrete nature of the image $f[\boldsymbol{k}]=\left.f(\boldsymbol{x})\right|_{\boldsymbol{x}=\boldsymbol{k} \in \mathbb{Z}^{2}}$, we work with the discrete version of $\tilde{f}(\boldsymbol{x})$ at $\boldsymbol{x}=\boldsymbol{k} \in \mathbb{Z}^{2}$ in Eq. (2.1).

\subsubsection{Filter Design for Fingerprint Segmentation}

The features of interest in a fingerprint image are repeated (curved) patterns which are concentrated in a particular range of frequencies after a Fourier transformation. In principle, the frequencies lower than these range's limits correspond to homogeneous regions and those higher to small scale objects, i.e. noise, respectively. Taking this prior knowledge into account, we design an algorithm that captures these fingerprint patterns in different directional subbands in the frequency domain for extracting the texture.

In this section, we design angularpass and bandpass filters. The angularpass filter builds on iterates of the directional Hilbert transformation, a multidimensional generalization of the Hilbert transform called the Riesz transform. It can be represented via principal value convolution kernels. The bandpass filter builds on the Butterworth transform which can be represented directly via a convolution kernel. We follow here a standard technique designing a bandpass filter from a lowpass filter which has an equivalent representation in analog circuit design. 


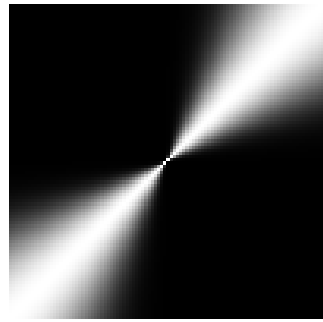

(a) $\widehat{h}_{l}^{n}(\boldsymbol{\omega})$

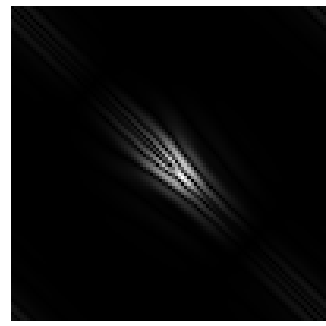

(e) $\operatorname{Re}\left\{\phi_{l}^{\gamma, n}(\boldsymbol{x})\right\}$

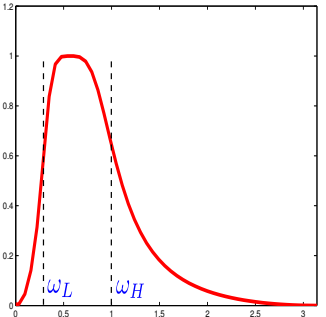

(b) $1 \mathrm{D}: \widehat{g}^{\gamma}(\omega)$

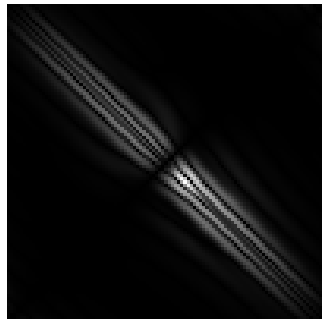

(f) $\operatorname{Im}\left\{\phi_{l}^{\gamma, n}(\boldsymbol{x})\right\}$

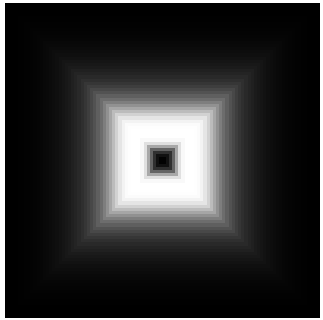

(c) $2 \mathrm{D}: \widehat{g}^{\gamma}(\boldsymbol{\omega})$

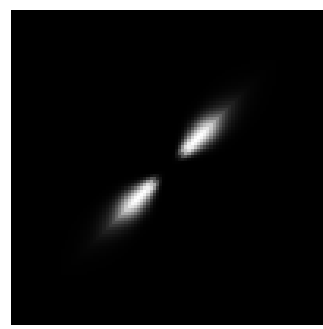

(g) $\left|\widehat{\phi}_{l}^{\gamma, n}(\boldsymbol{\omega})\right|^{2}$

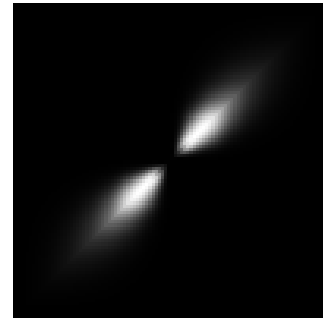

(d) $\hat{\phi}_{l}^{\gamma, n}(\boldsymbol{\omega})$

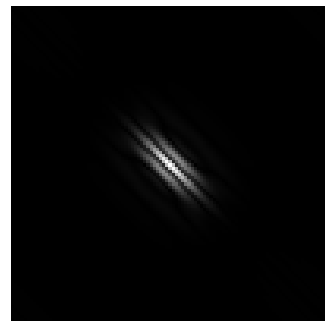

(h) $\left(\phi_{l}^{\gamma, n} * \phi_{l}^{\gamma, n, \vee}\right)(\boldsymbol{x})$

Figure 2.2: Image (a) displays the angularpass filter $\widehat{h}_{l}^{n}(\boldsymbol{\omega})$ with $\theta=13 \pi / 16, n=20$. Images (b-c) show the 1D and 2D Butterworth bandpass filters $\widehat{g}^{\gamma}(\omega)$ and $\widehat{g}^{\gamma}(\boldsymbol{\omega})$ with $\omega_{L}=0.3, \omega_{H}=1, \gamma=2$, (d) the spectrum of the DHBB filter $\widehat{\phi}_{l}^{\gamma, n}(\boldsymbol{\omega})$. Images (e-f) visualize the real and imaginary part of the DHBB filter $\phi_{l}^{\gamma, n}(\boldsymbol{x})$. Images (g-h) display the squared magnitude of the spectrum of the DHBB in the frequency and spatial domains which acts somewhat like a Gabor filter.

\subsubsection{The $n^{\text {th }}$ Order Directional Hilbert Transform of a Butterworth Bandpass}

Although a fingerprint image

$$
\boldsymbol{k}=\left[k_{1}, k_{2}\right] \mapsto f[\boldsymbol{k}], \quad\{-M, \ldots, M] \times\{-N, \ldots, N\} \rightarrow\{0, \ldots, 255\}
$$

is a discrete signal observed over a discrete grid, $M, N \in \mathbb{N}$ we start our considerations with a signal

$$
\boldsymbol{x}=\left(x_{1}, x_{2}\right) \mapsto f(\boldsymbol{x}), \quad D:=[-a, a] \times[-b, b] \rightarrow[0,1]
$$

assuming values in a continuum $a, b>0$. The frequency coordinates in the spectral domain will be denoted by $\boldsymbol{\omega}=\left(\omega_{1}, \omega_{2}\right) \in \mathbb{R}^{2}$.

As usual, the following operators are defined first for functions $f$ in the Schwartz Space of rapidly-decaying and infinitely differentiable test functions:

$$
S\left(\mathbb{R}^{d}\right)=\left\{f \in C^{\infty}\left(\mathbb{R}^{d}\right)\left|\sup _{\boldsymbol{x} \in \mathbb{R}^{d}}\right|\left(1+|\boldsymbol{x}|^{m}\right) \frac{d^{n}}{d \boldsymbol{x}^{n}} f(\boldsymbol{x}) \mid<+\infty, \quad \forall m, n \in \mathbb{Z}_{+}\right\},
$$

and continuously extended onto

$$
L_{2}\left(\mathbb{R}^{d}\right)=\left\{\left.f \in S\left(\mathbb{R}^{d}\right)\left|\|f\|_{L_{2}}=\int_{\mathbb{R}^{d}}\right| f(\boldsymbol{x})\right|^{2} d \boldsymbol{x}<+\infty\right\} .
$$


In our context we only need $d=1,2$. Further, we denote the Fourier and its inverse transformations by

$\mathcal{F}[f](\boldsymbol{\omega})=\int_{\mathbb{R}^{d}} f(\boldsymbol{x}) e^{-j\langle\boldsymbol{\omega}, \boldsymbol{x}\rangle} d \boldsymbol{x}=\widehat{f}(\boldsymbol{\omega}), \quad \mathcal{F}^{-1}[f](\boldsymbol{\omega})=\frac{1}{(2 \pi)^{d}} \int_{\mathbb{R}^{d}} \hat{f}(\boldsymbol{\omega}) e^{j\langle\boldsymbol{\omega}, \boldsymbol{x}\rangle} d \boldsymbol{x}$

where $j$ denotes the imaginary unit with $j^{2}=-1$.

Butterworth bandpass For $\gamma \in \mathbb{N}$ and frequency bounds $0<\omega_{L}<\omega_{H}$, setting $\Delta=$ $\omega_{H}-\omega_{L}, p^{2}=\omega_{H} \omega_{L}$, the one-dimensional $(d=1)$ Butterworth bandpass transform is defined via

$$
\mathcal{B}[f](x)=\mathcal{F}^{-1}[\omega \mapsto \underbrace{\sqrt{\frac{(\omega \Delta)^{2 \gamma}}{(\omega \Delta)^{2 \gamma}+\left(\omega^{2}-p^{2}\right)^{2 \gamma}}}}_{:=\hat{b}(\omega)} \hat{f}(\omega)](x),
$$

cf. [32]. It is easy to verify that $\hat{b}(\omega)$ tends to zero for $\omega \rightarrow 0$ and $\omega \rightarrow \infty$ and has unique maximum at the geometric mean $p$ with value 1 . In consequence, for high values of $\gamma$, this filter approximates the ideal filter

$$
\hat{b}_{\text {ideal }}(\omega)=\left\{\begin{array}{ll}
1 & \text { if } \omega_{L} \leq \omega \leq \omega_{H} \\
0 & \text { else }
\end{array} .\right.
$$

The ideal filter, however, suffers from the Gibbs effect. Letting $t=\frac{(j \omega)^{2}+p^{2}}{j \omega \Delta}$, we factorize the bandpass Butterworth as

$$
\hat{b}^{2}(\omega)=\frac{1}{(-1)^{\gamma}\left(t^{2}\right)^{\gamma}+1}=\frac{1}{(-1)^{\gamma} \prod_{k=1}^{\gamma}\left(t^{2}-t_{k}^{2}\right)}=\underbrace{\prod_{k=1}^{\gamma} \frac{1}{t-t_{k}}}_{H(t)} \cdot \underbrace{\prod_{k=1}^{\gamma} \frac{1}{-t-t_{k}}}_{H(-t)},
$$

with $t_{k}=e^{\pi j(\gamma+2 k-1) / 2 \gamma}(k=1, \ldots, \gamma)$, the negative squares of which representing the $\gamma$ different complex roots of $(-1)$. Then, with the below complex valued factor of $0 \leq \hat{b}^{2}(\omega)=B(j \omega) \cdot B(-j \omega)$ called the transfer function,

$$
H(t)=H\left(\frac{(j \omega)^{2}+p^{2}}{j \omega \Delta}\right)=\prod_{k=1}^{\gamma} \frac{\Delta(j \omega)}{(j \omega)^{2}-\Delta t_{k}(j \omega)+p^{2}}:=B(j \omega),
$$

we use the approximation: $j \omega=\log e^{j \omega} \approx 2 \frac{e^{j \omega}-1}{e^{j \omega}+1}$ to obtain

$$
B(j \omega) \approx \prod_{k=1}^{\gamma} \frac{2 \Delta\left(e^{2 j \omega}-1\right)}{\left(4+p^{2}-2 \Delta t_{k}\right) e^{2 j \omega}+\left(2 p^{2}-8\right) e^{j \omega}+\left(4+p^{2}+2 \Delta t_{k}\right)}:=B^{\gamma}\left(e^{j \omega}\right) .
$$

This approximation is often called the bilinear transform, which turns out to reduce the frequency bandwidth of interest, cf. Figure 2.7.

The $1 \mathrm{D}$ filter $B^{\gamma}\left(e^{i \omega}\right)$ is then generalized to a $2 \mathrm{D}$ domain. The McClellan transform [33], [34], [35], [36], would be one favorable method. Also, recently, bandpass filtering 
with a radial filter in the Fourier domain has been proposed by [37], [38] and [39] et al. for enhancing fingerprint images. However, for a simpler reconstruction of $2 \mathrm{D}$ filter and a data-friendly alternative to the polar tiling of the frequency plane, a Cartesian array is used instead (see [28], [29], [40], [41]).

Thus, on a rectangular domain $D=[-a, a] \times[-b, b]$ with common cuttoff frequencies $0<\omega_{L}<\omega_{H}$ and the two characteristic functions

$$
\chi_{h}\left(\omega_{1}, \omega_{2}\right):=\left\{\begin{array}{ll}
1 & \text { if } b\left|\omega_{1}\right| \geq a\left|\omega_{2}\right| \\
0 & \text { else }
\end{array} \quad, \quad \chi_{v}\left(\omega_{1}, \omega_{2}\right):= \begin{cases}1 & \text { if } b\left|\omega_{1}\right| \leq a\left|\omega_{2}\right| \\
0 & \text { else }\end{cases}\right.
$$

(see Figure 2.9), define

$$
\widehat{g}^{\gamma}\left(\omega_{1}, \omega_{2}\right)=B^{\gamma}\left(e^{j \omega_{1}}\right) \chi_{h}\left(\omega_{1}, \omega_{2}\right)+B^{\gamma}\left(e^{j \omega_{2}}\right) \chi_{v}\left(\omega_{1}, \omega_{2}\right)
$$

as the spectrum of our two-dimensional Butterworth filter $g^{\gamma}(\boldsymbol{x})$. Note that since $\widehat{g}^{\gamma}(\boldsymbol{\omega}) \in L_{2}\left(\mathbb{R}^{2}\right)$, there is a well defined $g^{\gamma}(\boldsymbol{x})=\mathcal{F}^{-1}\left[\widehat{g}^{\gamma}(\boldsymbol{\omega})\right](\boldsymbol{x})$.

Figure 2.2 (b) and 2.2 (c) show an example of the 1D and 2D Butterworth bandpass filters.

$n$-th order directional Hilbert transformations For more detail on the Hilbert transform $\mathcal{H}$ and the Riesz transform $\mathcal{R}$, we refer the reader to the literature for an in-depth discussion [25], [27], [42], [43], [44], [45, 46, 47], [48], and [49].

Consider a vector $\boldsymbol{u} \in \mathbb{R}^{d}$ and set and compute, respectively, for $\boldsymbol{x} \in \mathbb{R}^{d}$

$$
\begin{gathered}
\mathcal{R}[f](\boldsymbol{x}):=\mathcal{F}^{-1}[\boldsymbol{\omega} \mapsto \underbrace{-j \frac{\boldsymbol{\omega}}{\|\boldsymbol{\omega}\|}}_{:=\widehat{h}(\boldsymbol{\omega})} \hat{f}(\boldsymbol{\omega})](\boldsymbol{x}) \\
\mathcal{H}_{\boldsymbol{u}}[f](\boldsymbol{x}):=\langle\boldsymbol{u}, \mathcal{R}[f](\boldsymbol{x})\rangle=\mathcal{F}^{-1}[\boldsymbol{\omega} \mapsto \underbrace{-j \frac{\langle\boldsymbol{u}, \boldsymbol{\omega}\rangle}{\|\boldsymbol{\omega}\|}}_{:=\widehat{h}_{\boldsymbol{u}}(\boldsymbol{\omega})} \hat{f}(\boldsymbol{\omega})](\boldsymbol{x}) \\
\underbrace{\mathcal{H}_{\boldsymbol{u}} \ldots \mathcal{H}_{\boldsymbol{u}}}_{n-\text { times }}[f](\boldsymbol{x})=\mathcal{H}_{\boldsymbol{u}}^{n}[f](\boldsymbol{x})=\mathcal{F}^{-1}[\boldsymbol{\omega} \mapsto \underbrace{(-j)^{n} \frac{\langle\boldsymbol{u}, \boldsymbol{\omega}\rangle^{n}}{\|\boldsymbol{\omega}\|^{n}}}_{\widehat{h}_{\boldsymbol{u}}^{n}(\boldsymbol{\omega})} \hat{f}(\boldsymbol{\omega})](\boldsymbol{x}), n \in \mathbb{N}(2.4)
\end{gathered}
$$

The first line (2.3) called the Riesz transform has a representation as a principal value integral

$$
\mathcal{R}[f](\boldsymbol{x})=\left(f *_{p v} h\right)(\boldsymbol{x})=\lim _{\epsilon \rightarrow 0} \int_{\|\boldsymbol{y}-\boldsymbol{x}\| \geq \epsilon} f(\boldsymbol{x}-\boldsymbol{y}) h(\boldsymbol{y}) d \boldsymbol{y}
$$

where

$$
h(\boldsymbol{y}):=\left\{\begin{aligned}
\frac{\boldsymbol{y}}{\|\boldsymbol{y}\|^{d+1} \frac{\Gamma\left(\frac{d+1}{2}\right)}{\pi^{\frac{d+1}{2}}}} & \text { for } d>1 \\
\frac{1}{\pi y} & \text { for } d=1
\end{aligned}\right.
$$


Setting $h_{\boldsymbol{u}}(\boldsymbol{y})=\langle\boldsymbol{u}, h(\boldsymbol{y})\rangle$ and $h_{\boldsymbol{u}}^{n}(\boldsymbol{y})=\left(h_{\boldsymbol{u}} *_{p v} \ldots *_{p v} h_{\boldsymbol{u}}\right)(\boldsymbol{y})$ we haven for the third line (2.4) called the $n$-order directional Hilbert transform that

$$
\mathcal{H}_{\boldsymbol{u}}^{n}[f](\boldsymbol{x})=f *_{p v} h_{\boldsymbol{u}}^{n}(\boldsymbol{x}) .
$$

Since $-1 \leq \frac{\langle\boldsymbol{u}, \boldsymbol{\omega}\rangle}{\|\boldsymbol{\omega}\|} \leq 1$ and high powers preserve the values near \pm 1 while forcing all other values in $(-1,1)$ towards 0 , this filter gives roughly the same result as an inverse Fourier transform of a convolution of the signal's Fourier transform with

$$
A_{\alpha, \boldsymbol{u}}(\boldsymbol{\omega})= \begin{cases}1 & \text { if } \frac{|\langle\boldsymbol{u}, \boldsymbol{\omega}\rangle|}{\|\boldsymbol{\omega}\|} \geq \cos \alpha \\ 0 & \text { else }\end{cases}
$$

for small $\alpha>0$. The directional Hilbert transform, however, suffers less from a Gibbs effect than this sharp cutoff filter.

In $2 \mathrm{D}$, the direction vector is $\boldsymbol{u}=[\cos (\theta), \sin (\theta)]^{T}$ with the discretized $\theta=\frac{\pi l}{L} \in[0, \pi)$ and $l=0,1, \ldots, L-1$, where $L \in \mathbb{N}$ is the total number of orientation. Rewrite the impulse response of the $n$-th $\in \mathbb{N}$ order directional Hilbert transform in (2.4) as

$$
\widehat{h}_{\boldsymbol{u}}^{n}\left(\omega_{1}, \omega_{2}\right)=\left[-j \cos \left(\tan _{2}^{-1}\left(\frac{\omega_{2}}{\omega_{1}}\right)-\frac{\pi l}{L}\right)\right]^{n}:=\widehat{h}_{l}^{n}\left(\omega_{1}, \omega_{2}\right) .
$$

Putting together (Eq. (2.2), (2.4), (2.5)), for a fixed bandpass $\omega_{L}<\omega_{H}$ and $L$ directional subbands we have thus the DHBB filter of order $\gamma, n$ :

$$
\mathcal{H}_{u}^{n}\left[g^{\gamma}\right](\boldsymbol{x})=\mathcal{F}^{-1}[\boldsymbol{\omega} \mapsto \underbrace{\widehat{h}_{l}^{n}(\boldsymbol{\omega}) \cdot \widehat{g}^{\gamma}(\boldsymbol{\omega})}_{:=\widehat{\phi}_{l}^{\gamma, n}(\boldsymbol{\omega})}](\boldsymbol{x}):=\phi_{l}^{\gamma, n}(\boldsymbol{x}) .
$$

\subsubsection{Thresholding}

For given $\beta>0$, soft-thresholding is defined as follows

$$
x \mapsto \mathbf{T}(x, \beta)=\frac{x}{|x|} \cdot \max (|x|-\beta, 0) .
$$

Thus, the thresholded coefficients are $d_{l}[\boldsymbol{m}]=\mathbf{T}\left\{c_{l}[\boldsymbol{m}], \beta\right\}$. Note that $d_{l}[\boldsymbol{m}]$ is a solution of the $\ell_{1}$-shrinkage minimization problem

$$
\min _{u}\left\{\beta\|u\|_{\ell_{1}}+\frac{1}{2}\left\|u-c_{l}\right\|_{\ell_{2}}^{2}\right\}
$$

yielding soft-thresholding (cf. [50]). Figure 2.4 visualizes the effect of the soft-thresholding and the comparison with the others (such as: hard [50], semi-soft [51] and nonlinear [52] thresholding operators).

\subsubsection{Fingerprint Segmentation}

After having designed the FDB filter, let us now ponder on parameter selection, image binarization and morphological processing. 


\subsubsection{Parameter Choice for Texture Extraction}

A fingerprint image will be rescaled such that its oscillation pattern stays in a specific range in the Fourier domain, the coordinates of which are $\omega_{i}=[-\pi, \pi], i \in\{1,2\}$. For choosing the cutoff frequencies $\omega_{L}$ and $\omega_{H}$, we incorporate our prior knowledge about adult fingerprint images at resolution of 500 DPI: Valid interridge distances remain in a known range approximately from 3 to 25 pixels [11]. This corresponds exactly to $\omega_{H}=1$ as a limit for high frequencies. A limit of $\omega_{L}=0.3$ for low frequencies of the Butterworth bandpass filter corresponds to an interridge distance of about 12 pixels. The range $\left|\omega_{i}\right| \in\left[\omega_{H}, \pi\right]$ contains the small scale objects which are considered as noise. The range $\left|\omega_{i}\right| \in\left[0, \omega_{L}\right]$ contains the low frequency objects, corresponding to homogeneous regions.

The number of directions $L$ in and the order $n$ of the directional Hilbert transform involves a tradeoff between the following effects. We observe that with increased order $n$ the filter's shape becomes thinner in the Fourier domain. Although this sparsity smooths the texture image in the spatial domain, in order to fully cover all FOTIs, $L$ needs to grow with $n$. However, a disadvantage of choosing large $n$ and $L$ is that errors occur on the boundary due to the over-smoothing effect as illustrated in Figure 2.5 (o).

The next parameter to select is the order of the Butterworth filter $\gamma$. An illustration of the filter for different orders $\gamma \in\{1,2,3,10\}$ and with cutoff frequencies $\omega_{L}=0.3$ and $\omega_{H}=1$ is shown in Figure 2.6, its bilinear approximation in Figure 2.7. As $\gamma$ increases the filter becomes sharper. For very large values of $\gamma$, it approaches the ideal filter which is known to cause the unfavorable Gibbs effect.

The thresholding value $\beta$ separates large coefficients corresponding to the fingerprint pattern (FOTIs) (which are slightly attenuated due to soft-thresholding) from small coefficients corresponding to non-FOTIS and FOTIs which are not features due to the fingerprint pattern (these are eliminated). On the one hand, if $\beta$ is chosen too large, more prominent parts of true fingerprint tend to be removed. On the other hand, if $\beta$ is chosen too small, not all all unwanted features (as above) are removed which may cause segmentation errors.

In order to find good trade-offs, as described above, $n, L, \gamma$ and $\beta$ are trained as described in Section 2.3.1. In fact, since different fingerprint sensors have different properties, $\beta$ is adaptively adjusted to the intensity of coefficients in all subbands as

$$
\beta=C \cdot \max _{l, \boldsymbol{m}}\left\{c_{l}[\boldsymbol{m}]\right\} .
$$

Thus, instead of $\beta, C$ is trained for each sensor.

\subsubsection{Texture Binarization}

In the first step, the texture is decomposed by the operator (2.1) to obtain the reconstructed image $\tilde{f}[\boldsymbol{k}]$. Then, $\tilde{f}[\boldsymbol{k}]$ is binarized using an adaptive threshold adjusted to the intensity of $\tilde{f}[\boldsymbol{k}]$. Thus, the threshold is chosen as $C \cdot \max _{\boldsymbol{k}}(\tilde{f}[\boldsymbol{k}])$, with $C$ from $(2.8)$. If $\tilde{f}[\boldsymbol{k}]$ is larger than this threshold, it will be set to 1 (foreground), otherwise, it is set 
to 0 (background) as illustrated in Figure 2.1.

$$
\tilde{f}_{\text {bin }}[\boldsymbol{k}]= \begin{cases}1, & \tilde{f}[\boldsymbol{k}] \geq C \cdot \max _{\boldsymbol{k}}(\tilde{f}[\boldsymbol{k}]), \quad \forall \boldsymbol{k} \in \Omega \\ 0, & \text { else }\end{cases}
$$

\subsubsection{Morphological Processing}

In this final phase, we apply mathematical morphology (see Chapter 13 in [53]), to decide for each pixel whether it belongs to the foreground or background. Firstly, at each pixel $\tilde{f}_{\text {bin }}\left[k_{1}, k_{2}\right] \in\{0,1\}$, we build an $s \times s$ block centered at $\left(k_{1}, k_{2}\right)$ and 8 neighboring blocks (cf. Figure 2.3). Then, for each block, we count the white pixels and check whether their number exceeds the threshold $\frac{s^{2}}{t}$ with another parameter $t>0$. If at least $b$ blocks are above threshold, the pixel $\left[k_{1}, k_{2}\right]$ is considered as foreground.

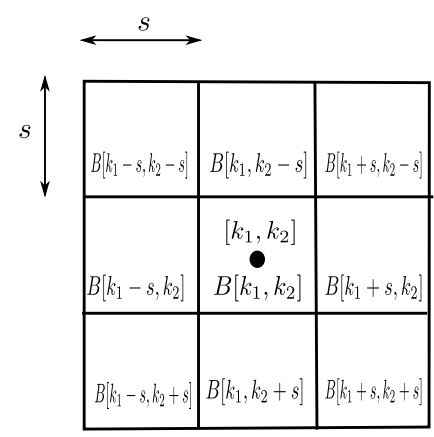

$$
\tilde{f}_{\text {dilate }}\left[k_{1}, k_{2}\right]= \begin{cases}1, & \#\left\{\sum B_{\left[k_{1}+m, k_{2}+m\right]} \geq \frac{s^{2}}{t}, m \in\{-s, 0, s\}\right\} \geq b \\ 0, & \text { else }\end{cases}
$$

Figure 2.3: The mor-

phological

element.

Then, the largest connected white pixel component is selected by a region filling method. Its convex hull is then the ROI. For better visualization we have inverted white and black, i.e. display the background by white pixels and the ROI by black pixels, cf. Figure 2.1.

\subsection{Evaluation Benchmark and Results}

The databases of FVC2000, 2002 and $2004[4,5,6]$ are publicly available and established benchmarks for measuring the verification performance of algorithms for image enhancement and fingerprint matching. Each competition comprises four databases: three of which contain real fingerprints acquired by different sensors and a database of synthetically generated images (DB 4 in each competition).

It has recently been shown that real and synthetic fingerprints can be discriminated with very high accuracy using minutiae histograms (MHs) [54]. More specifically, by computing the $\mathrm{MH}$ for a minutiae template and then computing the earth mover's distance (EMD) [55] between the template $\mathrm{MH}$ and the mean MHs for a set of real and synthetic fingerprints. Classification is simply performed by choosing the class with the smaller EMD. 
The nine databases containing real fingerprints have been obtained by nine different sensors and have different properties. The fingerprint image quality ranges from good quality images (especially FVC2002 DB1 and DB2) to low quality images which are more challenging to process (e.g. the databases of FVC2004). Some aspects of image quality concern both the segmentation step and the overall verification process, other aspects pose problems only for later stages of the fingerprint verification procedure, but have no influence on the segmentation accuracy.

Aspects of fingerprint image quality which complicate the segmentation:

- dryness or wetness of the finger

- a ghost fingerprint on the sensor surface

- small scale noise

- large scale structure noise

- image artifacts e.g. caused by reconstructing a swipe sensor image

- scars or creases interrupting the fingerprint pattern

Aspects of fingerprint image quality which make an accurate verification more difficult, but do not have any influence on the fingerprint segmentation step:

- distortion, nonlinear deformation of the finger

- small overlap area between two imprints

Each of the 12 databases contains 110 fingers with 8 impressions per finger. The training set consists of 10 fingers (80 images) and the test set contains 100 fingers (800 images). In total there are 10560 fingerprint images giving 10560 marked ground truth segmentations for training and testing.

\subsubsection{Experimental Results}

Segmentation Performance Evaluation Let $N_{1}$ and $N_{2}$ be the width and height of image $f[\boldsymbol{k}]$ in pixels. Let $M_{f}$ be number of pixels which are marked as foreground by human expert and estimated as background by an algorithm (missed/misclassified foreground). Let $M_{b}$ be number of pixels which are marked as background by human expert and estimated as foreground by an algorithm (missed/misclassified background). The average total error per image is defined as

$$
E r r=\frac{M_{f}+M_{b}}{N_{1} \times N_{2}} .
$$

The average error over 80 training images is basis for the parameter selection. In Table 2.3, we report the average error over all other 800 test images for each database and for each algorithms.

Parameter Selection Experiments were carried out on all 12 databases and are reported in Table 2.3. For each method listed in Table 2.3, the required parameters were trained on each of the 12 training sets: the choice of the threshold values for the Gabor filter bank based approach by Shen et al. [8], and the threshold values for the Harris 


\begin{tabular}{|c|l|}
\hline Parameters & Description \\
\hline$C$ & $\begin{array}{l}\text { a constant for selecting the threshold } \beta \text { in Eq. }(2.8) \\
\text { which removes small coefficients corresponding to noise. }\end{array}$ \\
\hline$n$ & $\begin{array}{l}\text { the order of the directional Hilbert transform which } \\
\text { corresponds to the angularpass filter in Eq. (2.4). }\end{array}$ \\
\hline$L$ & the number of orientations in the angularpass filter in Eq. (2.4). \\
\hline$\gamma$ & the order of the Butterworth bandpass filter in Eq. (2.2). \\
\hline$s$ & the window size of the block in the postprocessing step in Eq. (2.9). \\
\hline$t$ & a constant for selecting the morphology threshold $T$ in Eq. (2.9). \\
\hline$b$ & the number of the neighboring blocks in Eq. (2.9). \\
\hline
\end{tabular}

Table 2.1: Overview over all parameters for the factorized directional bandpass (FDB) method for fingerprint segmentation. Values are reported in Table 3.2.

\begin{tabular}{|c|c|c|c|c|}
\hline FVC & DB & $C$ & $\gamma$ & $t$ \\
\hline 2000 & 1 & 0.06 & 4 & 5 \\
& 2 & 0.07 & 2 & 5 \\
& 3 & 0.06 & 4 & 4 \\
& 4 & 0.03 & 1 & 5 \\
\hline 2002 & 1 & 0.04 & 1 & 4 \\
& 2 & 0.05 & 1 & 7 \\
& 3 & 0.09 & 1 & 5 \\
& 4 & 0.03 & 1 & 6 \\
\hline 2004 & 1 & 0.04 & 1 & 7 \\
& 2 & 0.08 & 2 & 5 \\
& 3 & 0.07 & 1 & 6 \\
& 4 & 0.05 & 1 & 5 \\
\hline
\end{tabular}

Table 2.2: Overview over the parameters learned on the training set. The other four parameters are $n=20, L=16, s=9$ and $b=6$ for all databases. 


\begin{tabular}{|c|c|c|c|c|l|l|}
\hline FVC & DB & GFB [8] & HCR [9] & MVC [7] & STFT [10] & FDB \\
\hline 2000 & 1 & 13.26 & 11.15 & 10.01 & 16.70 & 5.51 \\
& 2 & 10.27 & 6.25 & 12.31 & 8.88 & 3.55 \\
& 3 & 10.63 & 7.80 & 7.45 & 6.44 & 2.86 \\
& 4 & 5.17 & 3.23 & 9.74 & 7.19 & 2.31 \\
\hline 2002 & 1 & 5.07 & 3.71 & 4.59 & 5.49 & 2.39 \\
& 2 & 7.76 & 5.72 & 4.32 & 6.27 & 2.91 \\
& 3 & 9.60 & 4.71 & 5.29 & 5.13 & 3.35 \\
& 4 & 7.67 & 6.85 & 6.12 & 7.70 & 4.49 \\
\hline 2004 & 1 & 5.00 & 2.26 & 2.22 & 2.65 & 1.40 \\
& 2 & 11.18 & 7.54 & 8.06 & 9.89 & 4.90 \\
& 3 & 8.37 & 4.96 & 3.42 & 9.35 & 3.14 \\
& 4 & 5.96 & 5.15 & 4.58 & 5.18 & 2.79 \\
\hline Avg. & & 8.33 & 5.78 & 6.51 & 7.57 & 3.30 \\
\hline
\end{tabular}

Table 2.3: Error rates (average percentage of misclassified pixels averaged over 800 test images per database) computed using the manually marked ground truth segmentation and the estimated segmentation by these methods: a Gabor filter bank (GFB) response based method by Shen et al. [8], a Harris corner response (HCR) based approach by Wu et al. [9], a method by Bazen and Gerez using local gray-level mean, variance and gradient coherence (MVC) as features [7], a method applying short time Fourier transforms (STFT) by Chikkerur et al. [10] and the proposed method based on the factorized directional bandpass (FDB).

corner response based method by Wu et al. [9]. The parameters of the method by Bazen and Gerez are chosen as described in [7]: the window size of the morphology operator and the weights of the perceptron which are trained in $10^{4}$ iterations due to the large number of pixels in the training database. For the method of Chikkerur et al., we used the energy image computed by the implementation of Chikkerur, performed Otsu thresholding and mathematical morphology as explained in [53].

For the proposed FDB method, the involved parameters are summarized in Table 3.1 and the values of the learned parameters are reported in Table 3.2. Also, the mirror boundary condition with size 15 pixels is used in order to avoid boundary effects. In a reasonable amount of time, a number of conceivable parameter combinations were evaluated on the training set. The choice of these parameters balances the smoothing properties of the proposed filter attempting to avoid both under-smoothing and oversmoothing.

This systematic comparison of fingerprint segmentation methods clearly shows that the factorized directional bandpass method (FDB) outperforms the other four widely used segmentation methods on all 12 databases. An overview of visualized segmentation results by the FDB method is given in Figure 3.8. A few challenging examples for which the FDB method produces a flawed segmentation are depicted in Figure 3.15. Moreover, a comparison of all five segmentation methods and their main features for five example images are shown in Figure 2.14 to 2.18. 


\subsection{Conclusions}

In this chapter, we designed a filter specifically for fingerprints which is based on the directional Hilbert transform of Butterworth bandpass filters. A systematic comparison with four widely used fingerprint segmentation showed that the proposed FDB method outperforms these methods on all $12 \mathrm{FVC}$ databases using manually marked ground truth segmentation for the performance evaluation. The proposed FDB method for fingerprint segmentation can be combined with all methods for orientation field estimation like e.g. the line sensor method [56] or by a global model based on quadratic differentials [57] followed by liveness detection [58] or fingerprint image enhancement $[11,59]$. It can also be used in combination with alternative approaches, e.g. as a preprocessing step for locally adaptive fingerprint enhancement in the Fourier domain as proposed by Bartůněk et al. [22] or before applying structure tensor derived symmetry features for enhancement and minutiae extraction proposed by Fronthaler et al. [60].

Notably, the filter $\phi_{l}^{n, \gamma} * \phi_{l}^{n, \gamma, \vee}$ is similar to the Gabor filter which could have been used instead of the DHBB filter. Similarly, Bessel or Chebbychev transforms as well as B-splines as generalizations ([61]) could replace the Butterworth. We expect, however, for reasons elaborated, relying on the DHBB filter gives superior segmentation results.

The manually marked ground truth benchmark and the implementation of the FDB method are available for download at www.stochastik.math.uni-goettingen.de/ biometrics/.

In doing so, we would like to facilitate the reproducibility of the presented results and promote the comparability of fingerprint segmentation methods. 


\section{Supplementary Appendix}

\subsection{Comparison of the Operator in the FDB Method with the Summation and Maximum Operators}

We briefly illustrate the differences between the proposed FDB filter (2.1) and the maximum and summation operators for the coefficients in all directional subbands. Figure 2.10 compares the results of these operators for a low-quality and a good quality example. The functions are described as follows

- The maximum operator without and with the shrinkage operator (2.7) (depicted in the second and third row in Figure 2.10)

$$
\tilde{f}[\boldsymbol{k}]=\left\{\begin{array}{lc}
\max _{l}\left\{c_{l}[\boldsymbol{k}] \cdot\left(c_{l}[\boldsymbol{k}]>0\right)\right\}+\min _{l}\left\{c_{l}[\boldsymbol{k}] \cdot\left(c_{l}[\boldsymbol{k}]<0\right)\right\} & (\text { without }(2.7)) \\
\max _{l}\left\{d_{l}[\boldsymbol{k}] \cdot\left(d_{l}[\boldsymbol{k}]>0\right)\right\}+\min _{l}\left\{d_{l}[\boldsymbol{k}] \cdot\left(d_{l}[\boldsymbol{k}]<0\right)\right\} & (\text { with }(2.7)),
\end{array}\right.
$$

with $l=0,1, \ldots, L-1$.

- The summation operator without and with the shrinkage operator (2.7) (displayed in the fourth and fifth row in Figure 2.10)

$$
\tilde{f}[\boldsymbol{k}]= \begin{cases}\sum_{l=0}^{L-1} c_{l}[\boldsymbol{k}] & (\text { without }(2.7)) \\ \sum_{l=0}^{L-1} d_{l}[\boldsymbol{k}] & (\text { with }(2.7)) .\end{cases}
$$

\subsection{Additional Figures}



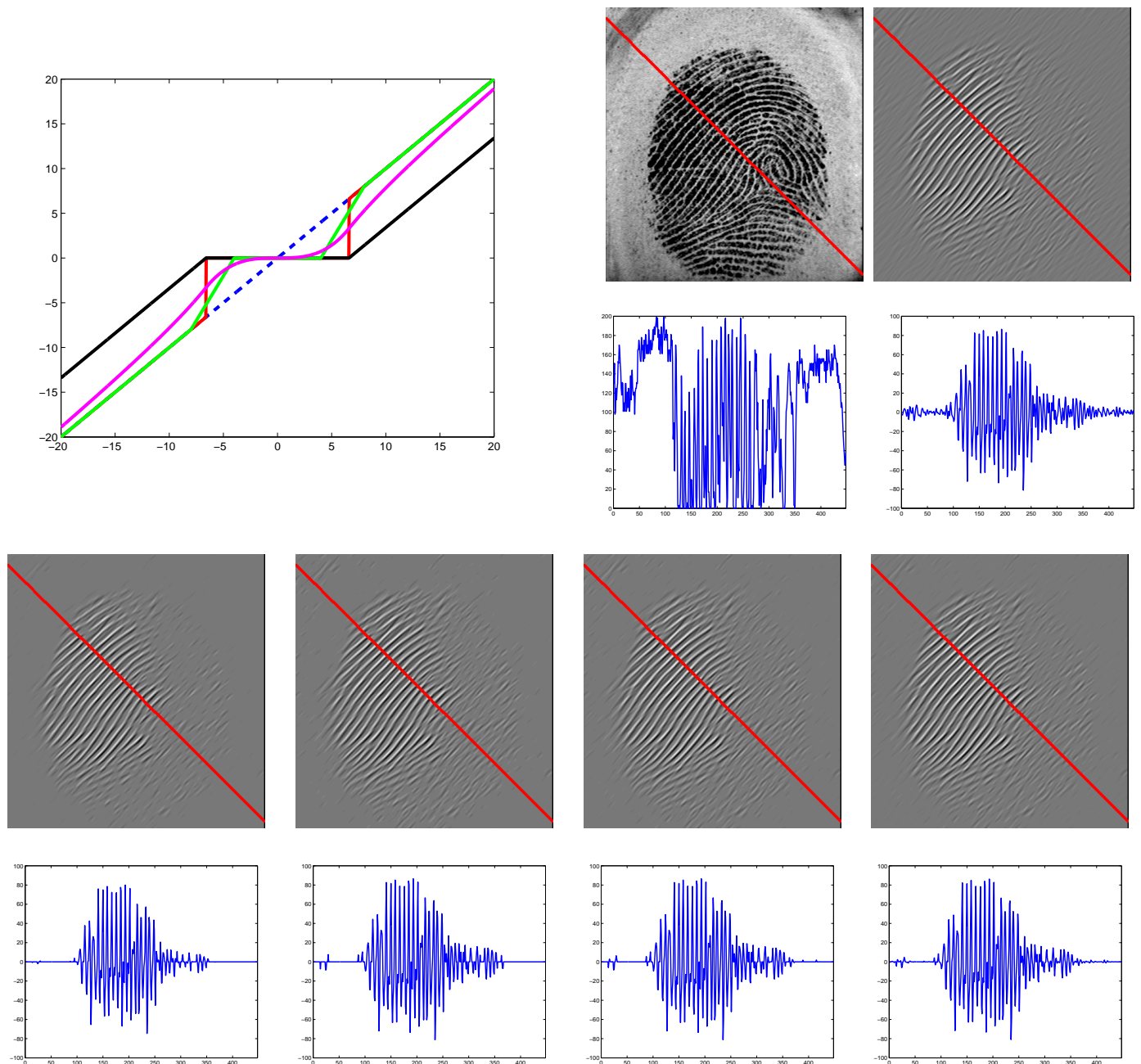

Figure 2.4: Four typical thresholding functions (red: hard, black: soft, green: semisoft, magenta: nonlinear) are compared (top left). The following six pairs show an image and the visualization of the corresponding 1D cross section along the red line. F.l.t.r and top to bottom: the original image $f[\boldsymbol{k}]$, the coefficient $c_{l}[\boldsymbol{k}]$ and the thresholded coefficients $d_{l}[\boldsymbol{k}]$ for the soft, hard, semisoft and nonlinear thresholding operators. Comparing the four cross sections in the bottom row, we observe that soft-thresholding achieves the sparsest solution. 


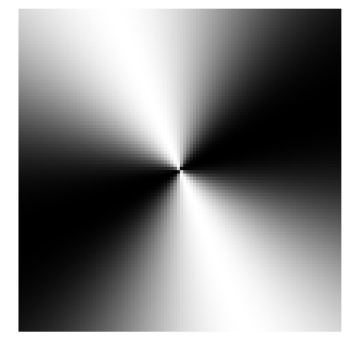

(a) $\widehat{h}_{l}^{n}(\boldsymbol{\omega}), n=3$

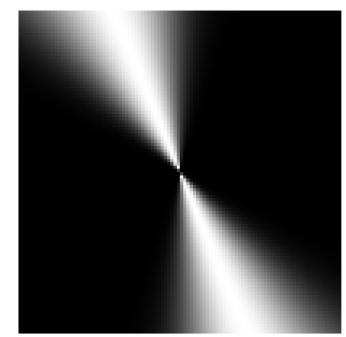

(e) $n=20$

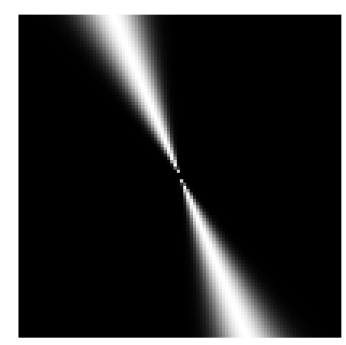

(i) $\mathrm{n}=100$

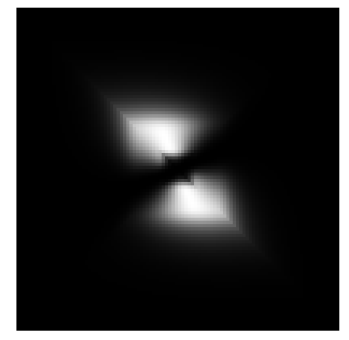

(b) $\widehat{\phi}_{l}^{\gamma, n}(\boldsymbol{\omega})$

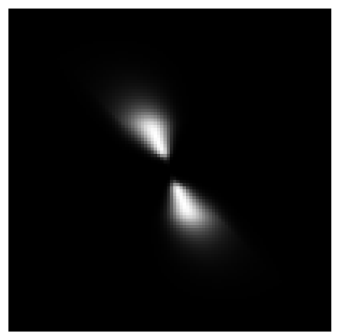

(f)

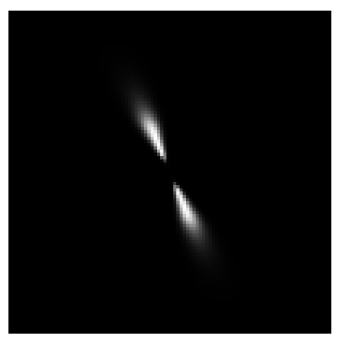

(j)

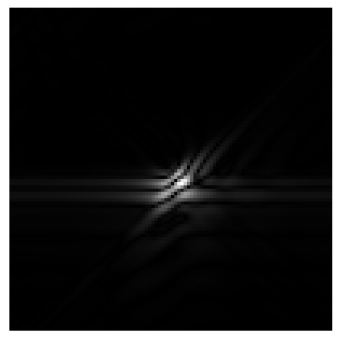

(c) $\left|\phi_{l}^{\gamma, n}(\boldsymbol{x})\right|$

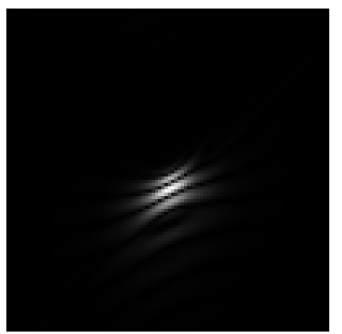

(g)

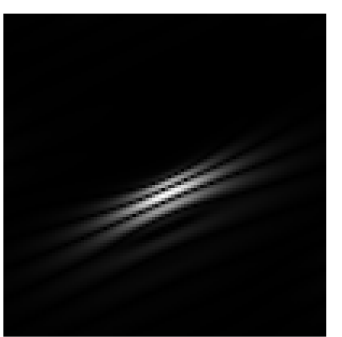

(k)

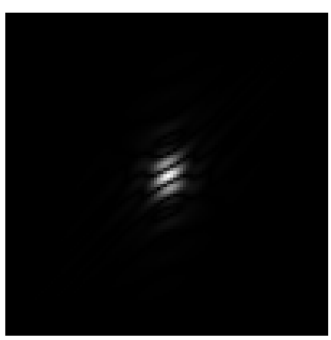

(d) $\left(\phi_{l}^{\gamma, n} * \phi_{l}^{\gamma, n, \vee}\right)(\boldsymbol{x})$

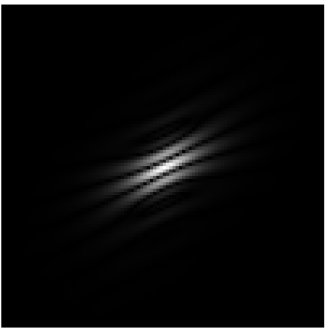

(h)

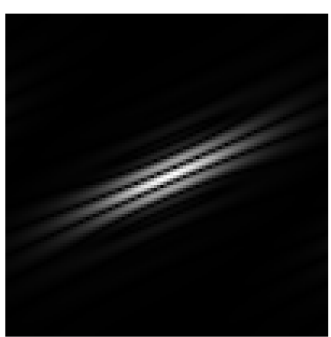

(1)

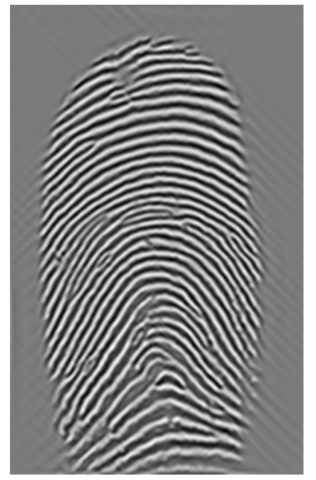

(m) $\mathrm{n}=3$

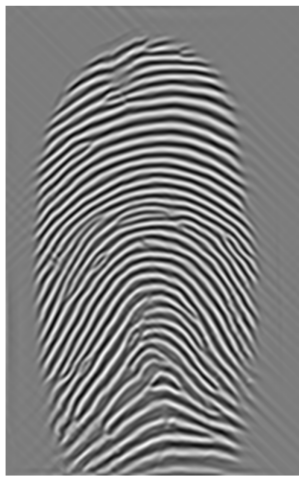

(n) $\mathrm{n}=20$

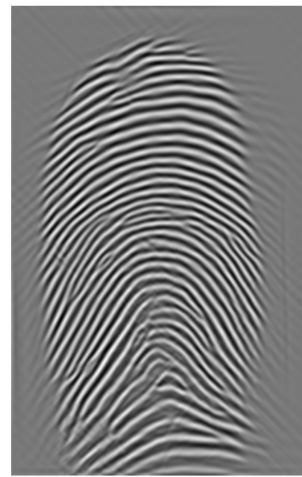

(o) $n=100$

Figure 2.5: Angular bandpass $\widehat{h}_{l}^{n}(\boldsymbol{\omega})$ at $\theta=\frac{7 \pi}{16}, \gamma=3$ and different orders $n \in$ $\{3,20,100\}$ and their responses (last row). 


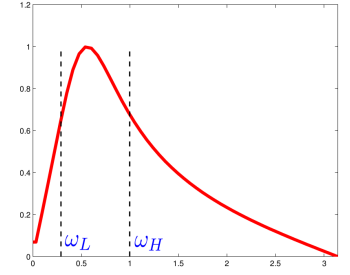

(a) $\gamma=1$
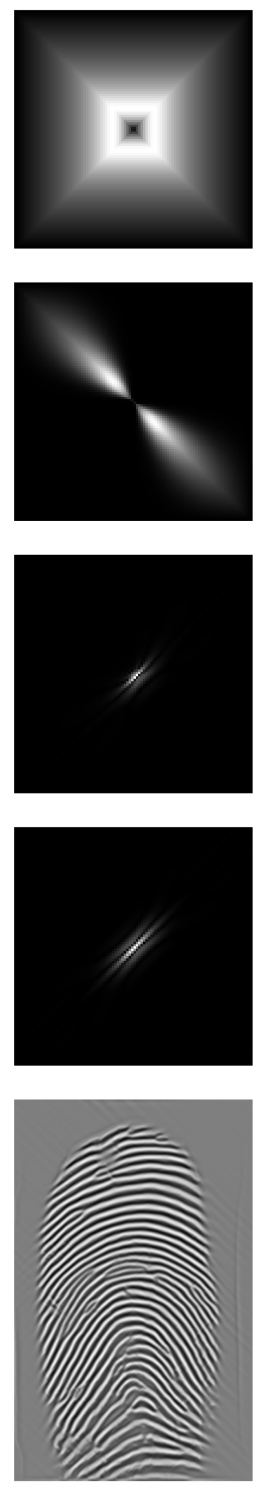

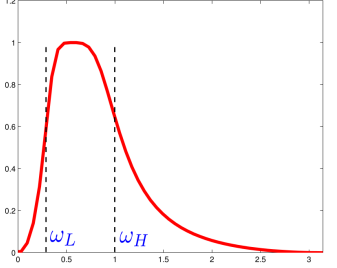

(b) $\gamma=2$
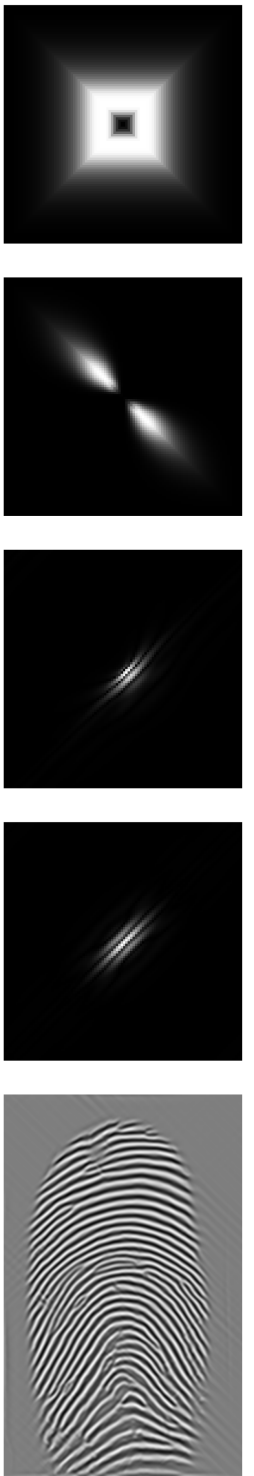

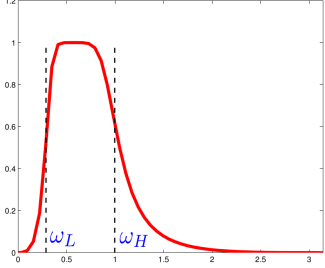

(c) $\gamma=3$
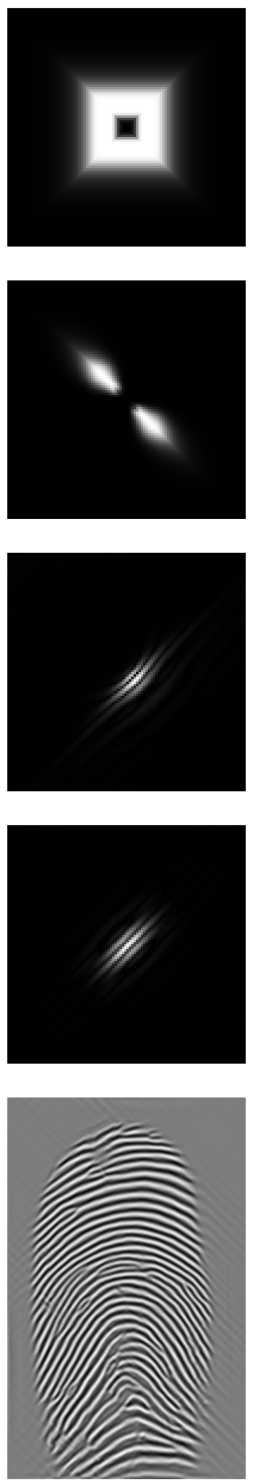

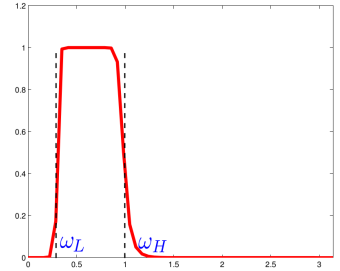

(d) $\gamma=10$
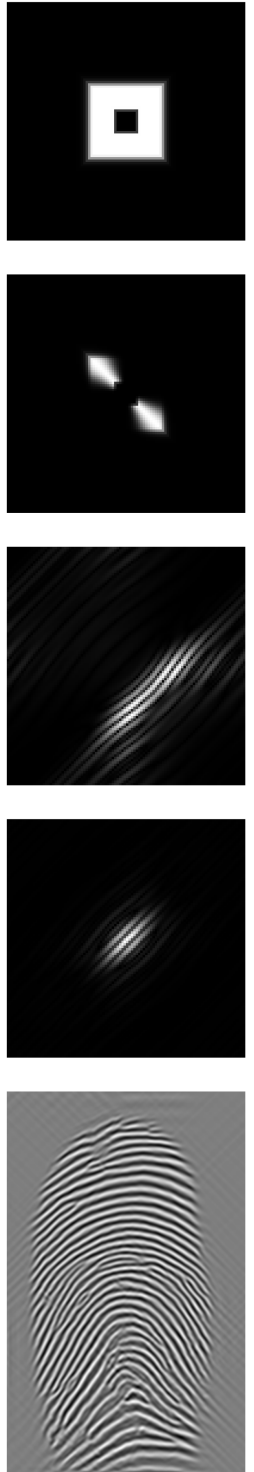

Figure 2.6: Butterworth bandpass filter $\widehat{g}^{\gamma}(\boldsymbol{\omega})$ at $\omega_{L}=0.3, \omega_{H}=1$ and different $\gamma$, angular bandpass filter with $n=20, L=16, \theta=\frac{5 \pi}{16}$, and their responses. $1^{\text {st }}$ row: 1D Butterworth, $2^{\text {nd }}$ row: 2D Butterworth, $3^{\text {rd }}$ row: $\widehat{\phi}_{l}^{\gamma, n}(\boldsymbol{\omega}), 4^{\text {th }}$ row: $\left|\phi_{l}^{\gamma, n}(\boldsymbol{x})\right|, 5^{\text {th }}$ row: $\left(\phi_{l}^{\gamma, n} * \phi_{l}^{\gamma, n, \vee}\right)(\boldsymbol{x}), 6^{\text {th }}$ row: their responses. 


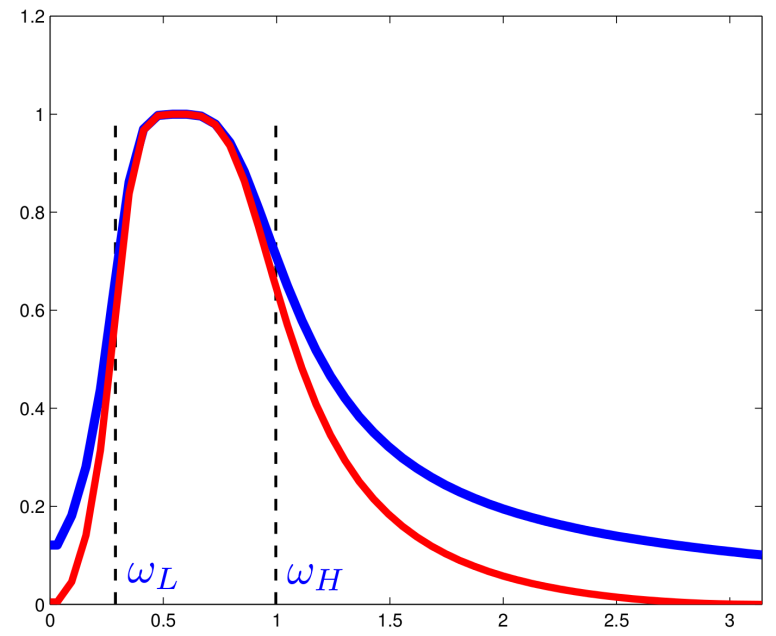

(a) $\widehat{g}^{\gamma}(\omega)$

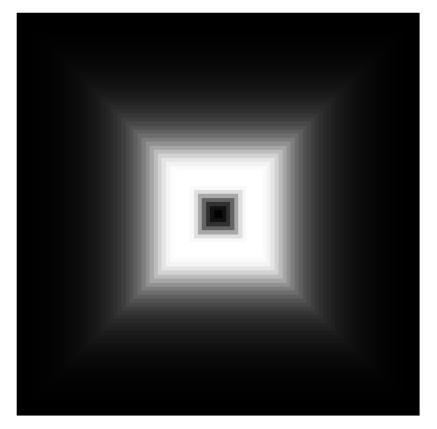

(b) $\widehat{g}^{\gamma}(\boldsymbol{\omega})$

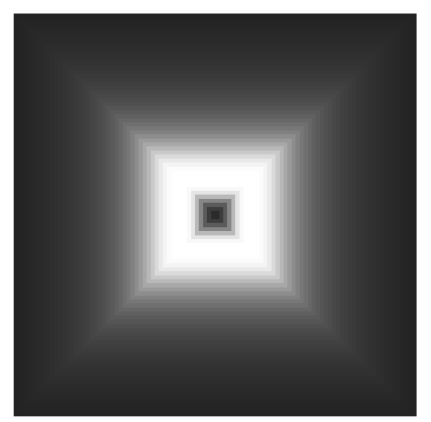

(e) $\widehat{g}^{\gamma}(\boldsymbol{\omega})$

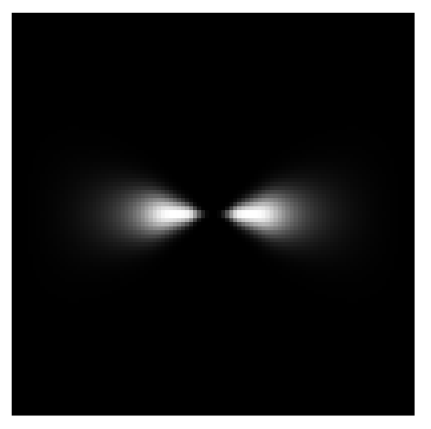

(c) $\widehat{\phi}_{l}^{\gamma, n}(\boldsymbol{\omega})$

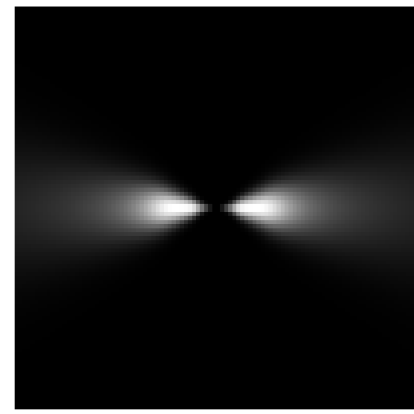

(f) $\widehat{\phi}_{l}^{\gamma, n}(\boldsymbol{\omega})$

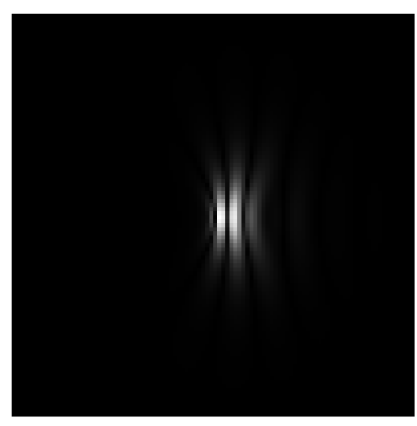

(d) $\left|\phi_{l}^{\gamma, n}(\boldsymbol{x})\right|$

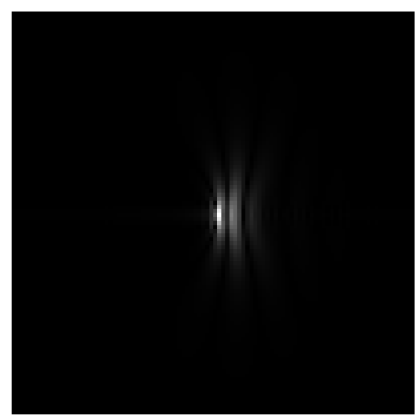

(g) $\left|\phi_{l}^{\gamma, n}(\boldsymbol{x})\right|$

Figure 2.7: Image (a) displays a 1D Butterworth bandpass filter (blue) and its approximation (red). Image (b) shows the 2D Butterworth bandpass filter $\widehat{g}^{\gamma}(\boldsymbol{\omega})$ at $n=20, \theta=0, \gamma=2$, and the corresponding DHBB filter in the Fourier and spatial domains (c, d) for the approximation by the bilinear transform. Image (e) visualizes the $2 \mathrm{D}$ version of the original filter and the corresponding DHBB filter (f, g). 


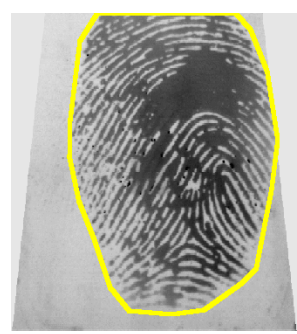

(a)

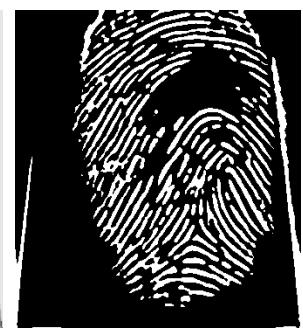

(b)

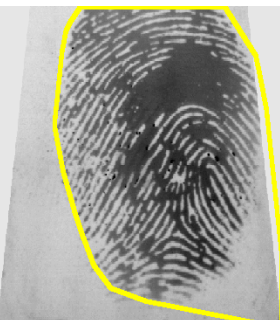

(c)

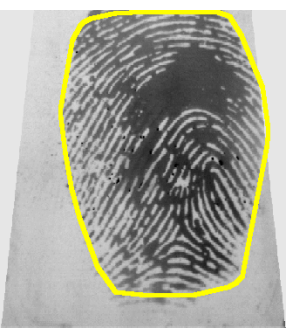

(d)

Figure 2.8: The ground truth segmentation (a) and the binarized texture image (b) for an example fingerprint. Applying a standard morphology operation like closing (dilation followed by erosion) instead of the proposed method connects in this example the white fingerprint texture with structure noise close to the margin of the texture and the result is a defective segmentation (c). The proposed morphology avoids this undesired effect by considering neighborhoods on two scales: cells of size $s \times s$ pixels and blocks of $3 \times 3$ cells.

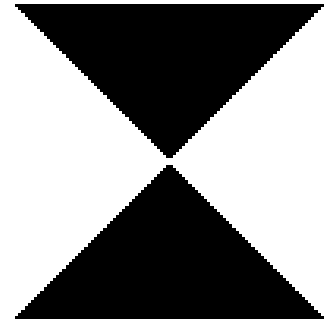

(a)

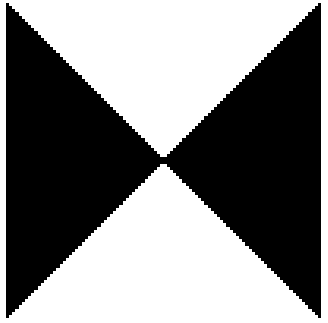

(b)

Figure 2.9: The indicator functions in the horizontal direction (a) and vertical direction (b). 


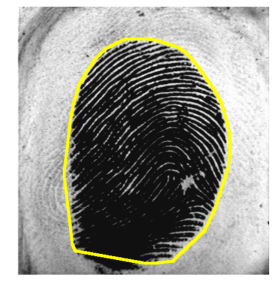

(a) $\operatorname{Err}=1.98$

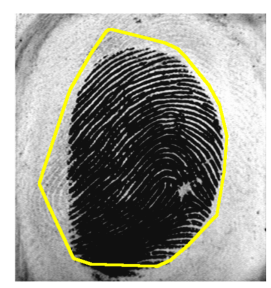

(e) $\operatorname{Err}=7.18$

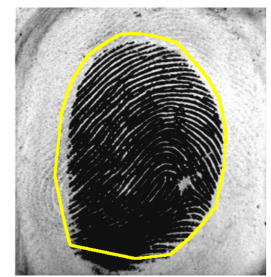

(i) $\operatorname{Err}=5.15$

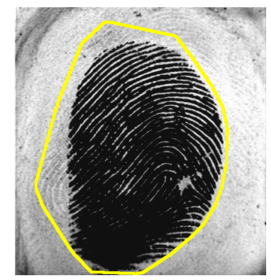

(m) $\operatorname{Err}=8.67$

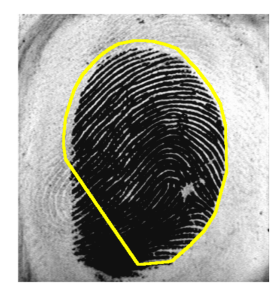

(q) $\operatorname{Err}=8.53$
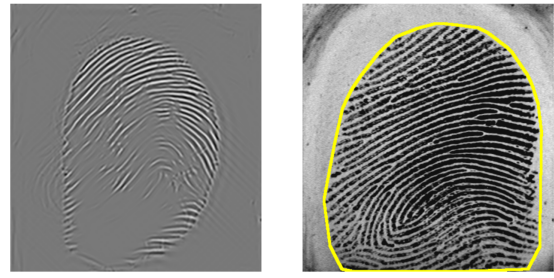

(c) Err $=1.44$
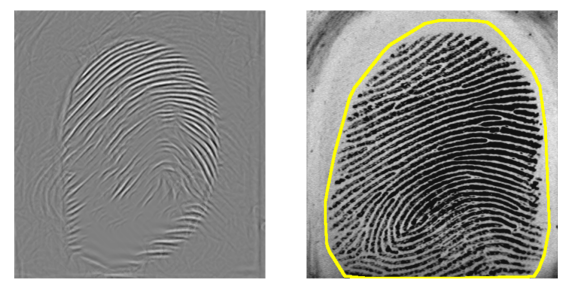

(g) $\operatorname{Err}=5.1$
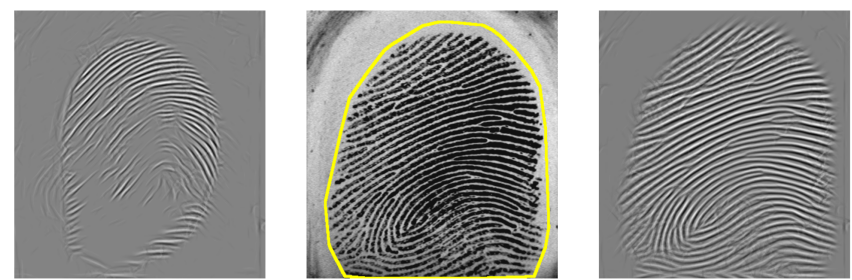

(k) $\operatorname{Err}=4.29$
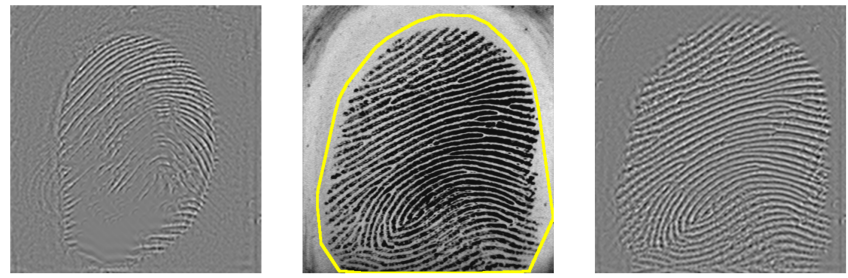

(o) $\operatorname{Err}=6.42$
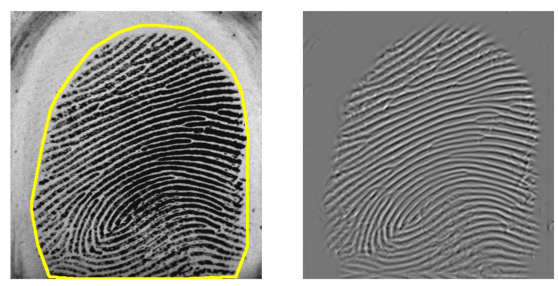

(s) $\operatorname{Err}=2.82$

Figure 2.10: Comparison of five image reconstruction strategies and their effect on the resulting segmentation. $1^{\text {st }}, 2^{\text {nd }}$ columns: segmented images (error in percent) and reconstructed images for a low-quality image and $3^{\text {rd }}, 4^{\text {th }}$ columns for a good quality image. $1^{\text {st }}$ row: the proposed operator. $2^{\text {nd }}, 3^{\text {rd }}$ rows: maximum operator without and with the shrinkage operator (2.7), respectively. $4^{\text {th }}, 5^{\text {th }}$ rows: summation operator without and with the shrinkage operator (2.7), respectively. 
CHAPTER 2. FILTER DESIGN AND PERFORMANCE EVALUATION FOR FINGERPRINT IMAGE SEGMENTATION
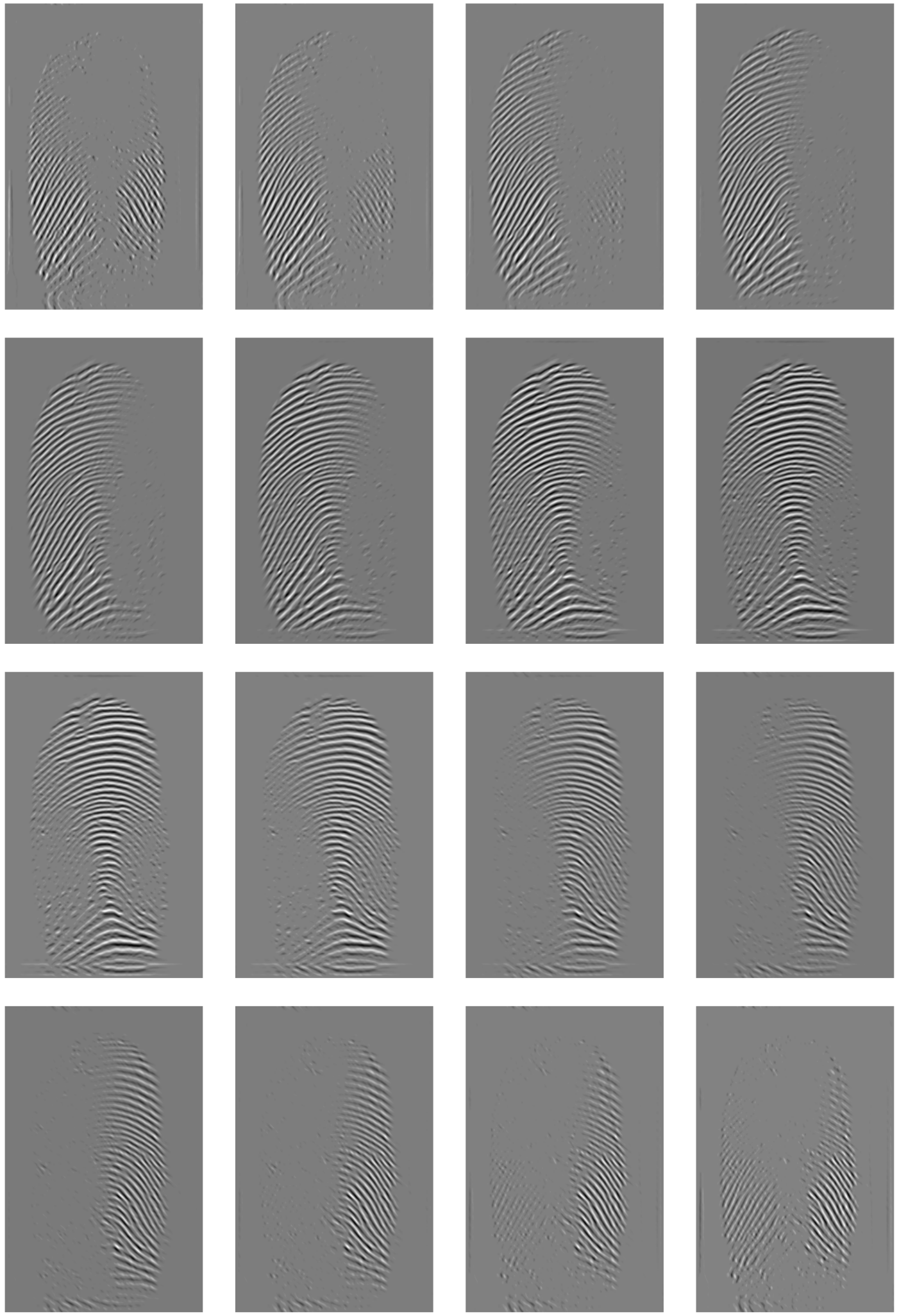

Figure 2.11: Visualization of the coefficients in the 16 subbands of the DHBB filter for $n=20, \gamma=3, \omega_{L}=0.3, \omega_{H}=1$. 

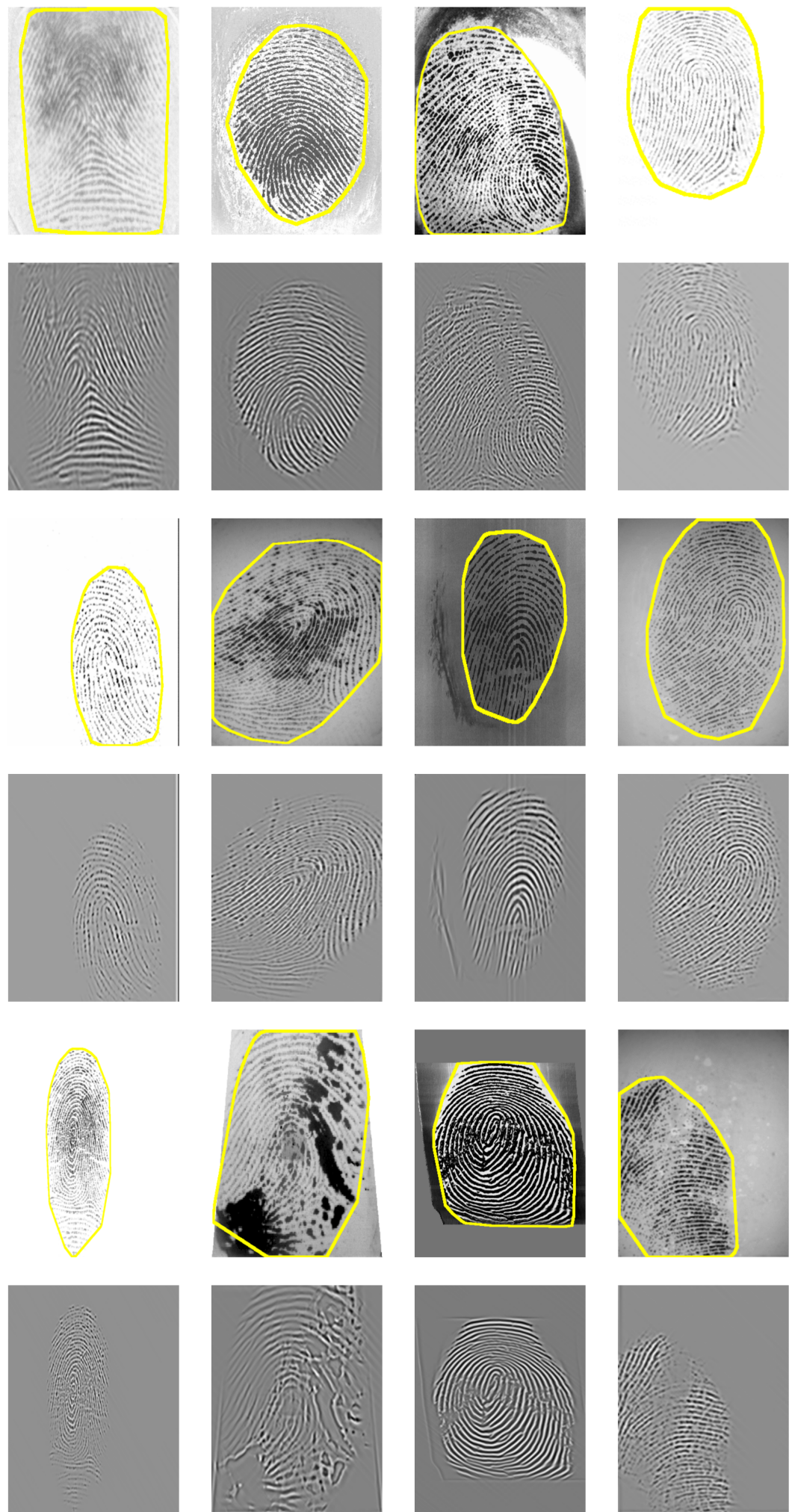

Figure 2.12: Segmented fingerprint images and the corresponding reconstructed texture images by the FDB method for FVC2000 (first and second row), FVC2002 (third and fourth row) and FVC2004 (fifth and sixth row). Columns f.l.t.r correspond to DB1 to DB4. 


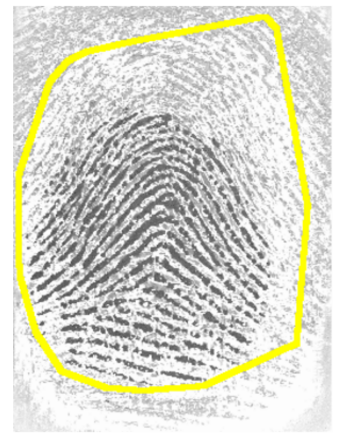

(a) $\operatorname{Err}=25.23$
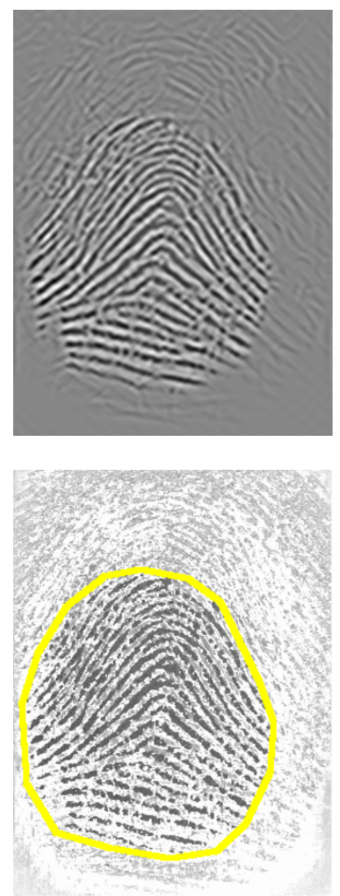

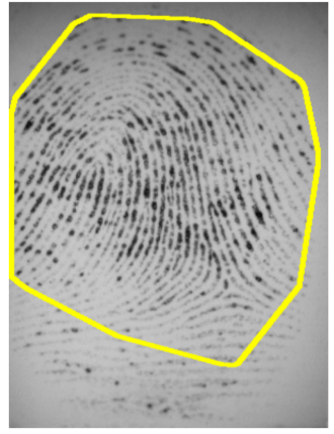

(b) $\operatorname{Err}=18.97$
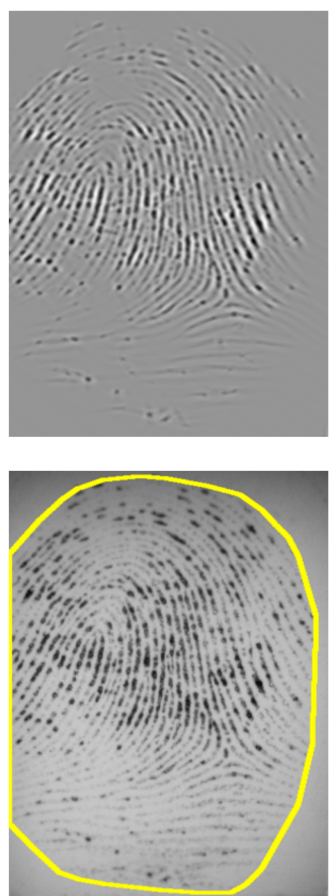

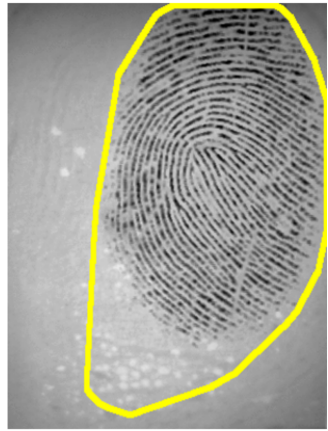

(c) $\operatorname{Err}=11.39$
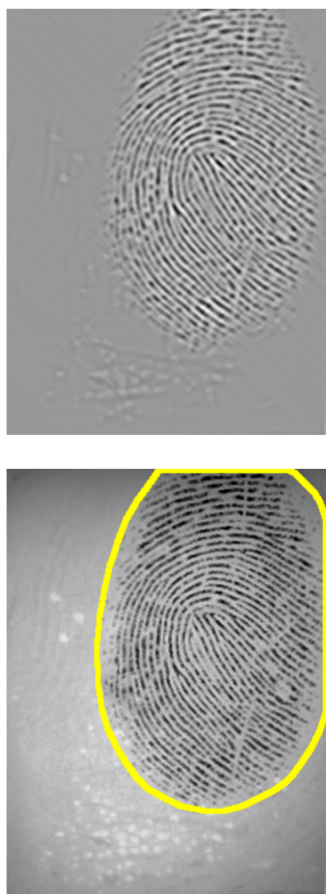

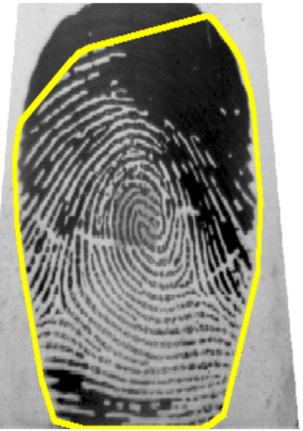

(d) $\operatorname{Err}=5.98$
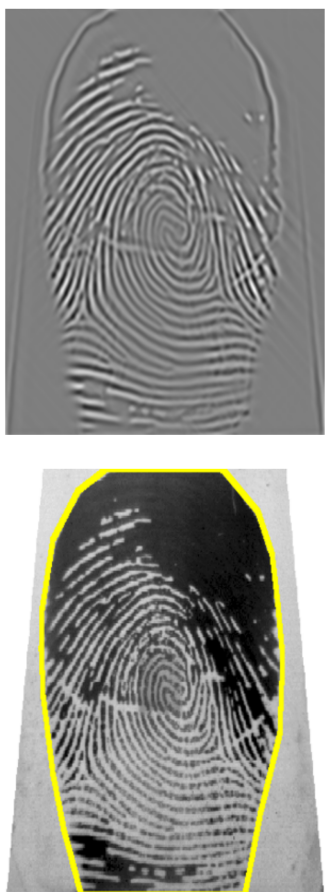

Figure 2.13: Examples of incorrectly segmented fingerprint images due to: (a) a ghost fingerprint on the sensor surface, (b) dryness of the finger, (c) texture artifacts in the reconstructed image, (d) wetness of the finger. The first row shows the segmentation obtained by the FDB method, the second row displays the reconstructed image and the last row visualizes the manually marked ground truth segmentation. 


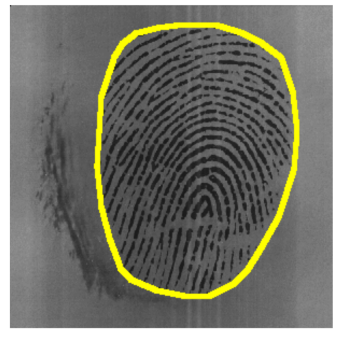

(a)

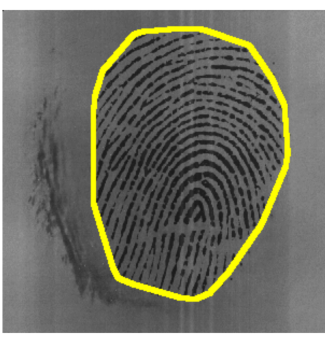

(b) $\operatorname{Err}=2.71$

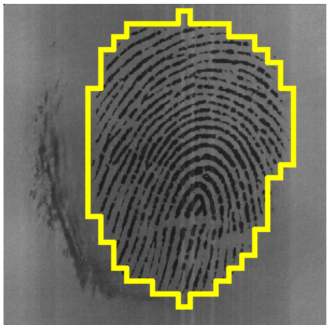

(f) $\operatorname{Err}=3.12$

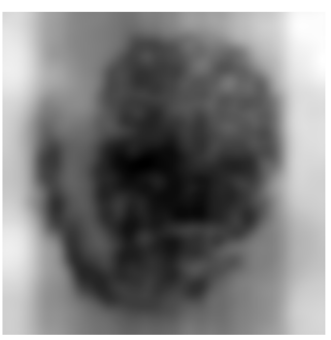

(j)

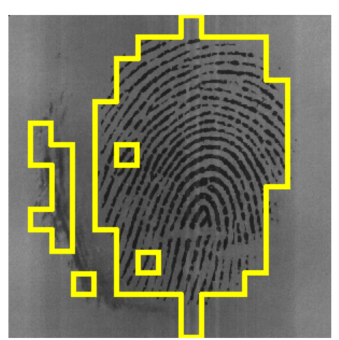

(c) $\operatorname{Err}=10.08$

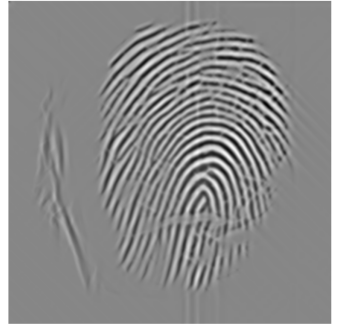

(g)

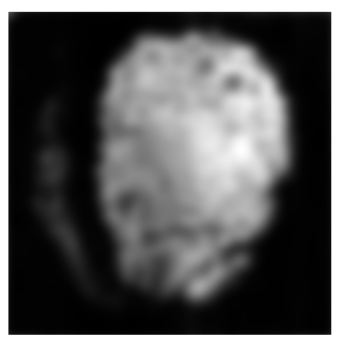

(k)

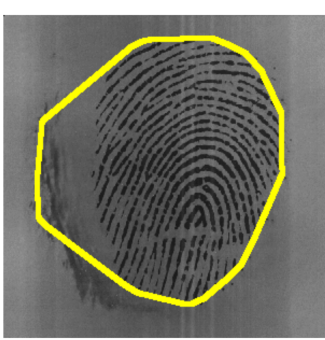

(d) $\operatorname{Err}=11.26$

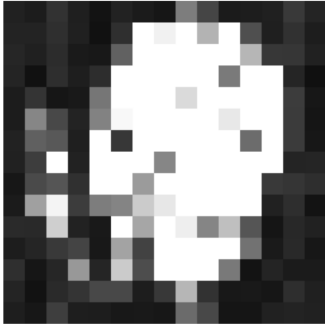

(h)

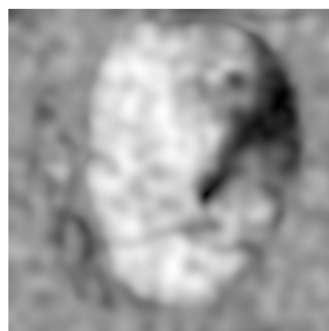

(1)

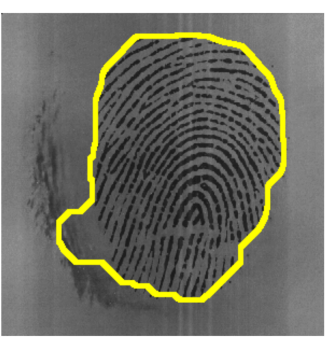

(e) $\operatorname{Err}=5.17$

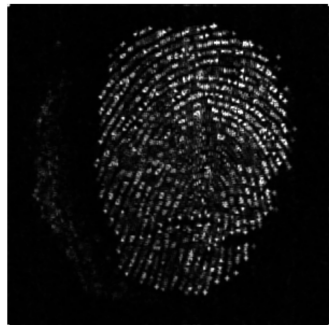

(i)

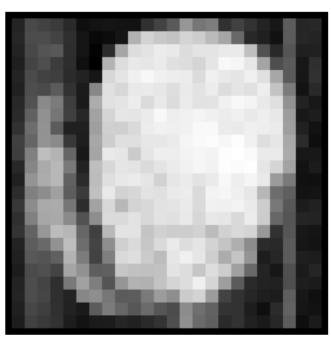

(m)

Figure 2.14: Segmented fingerprint images and their features of different methods for FVC2002_DB3_IM_15_1. (a) ground truth; (b, g) FDB, (c, h) Gabor, (d, i) Harris, (e, j, k, l) Mean-Variance-Coherence, (f, m) STFT. 
(a)

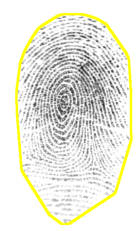

(b) $\mathrm{Err}=0.6$

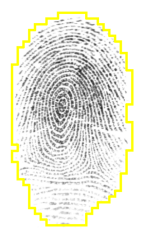

(f) $\mathrm{Err}=2.45$

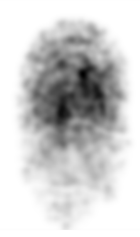

(j)

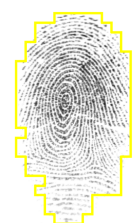

(c) Err $=2.08$

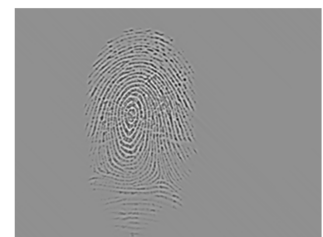

(g)

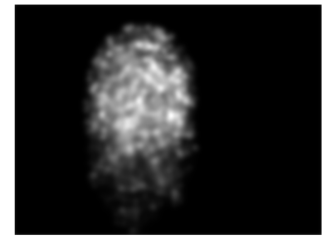

(k)

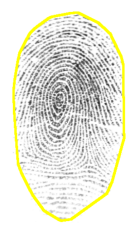

(d) Err $=1.34$

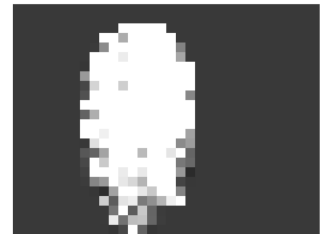

(h)

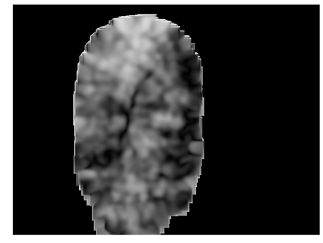

(1)

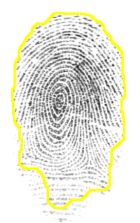

(e) Err $=3.87$

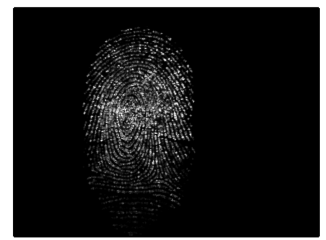

(i)

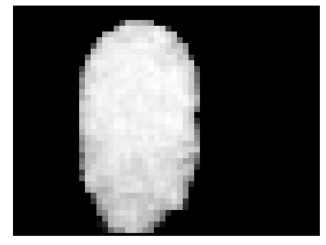

(m)

Figure 2.15: Segmented fingerprint images and their features of different methods for FVC2004_DB1_IM_24_7. (a) ground truth; (b, g) FDB, (c, h) Gabor, (d, i) Harris, (e, j, k, l) Mean-Variance-Coherence, (f, m) STFT. 


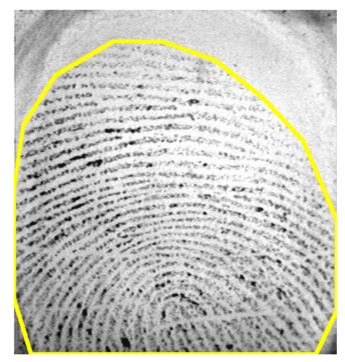

(a)

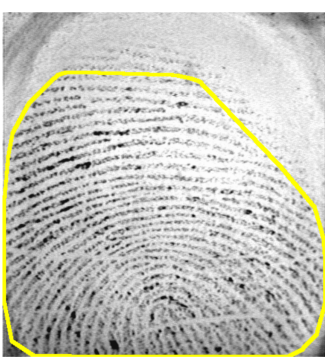

(b) $\operatorname{Err}=7.03$

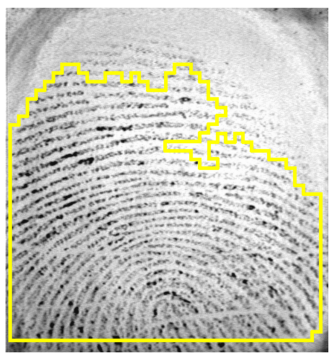

(f) $\operatorname{Err}=13.45$

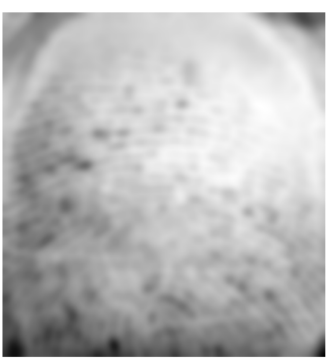

(j)

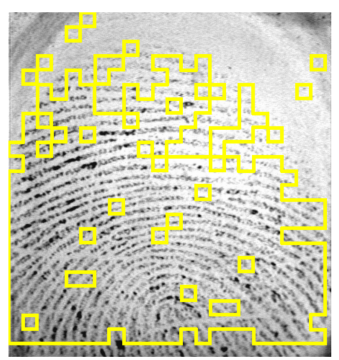

(c) $\operatorname{Err}=20.55$

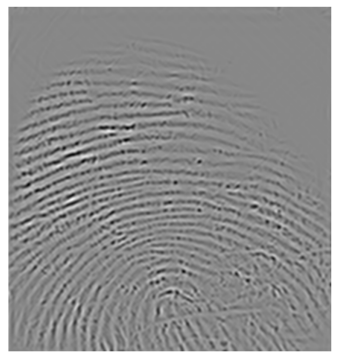

(g)

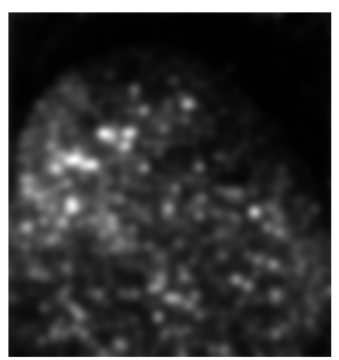

(k)

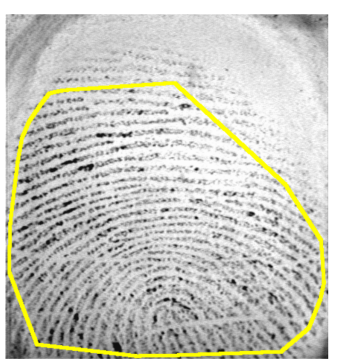

(d) $\operatorname{Err}=14.98$

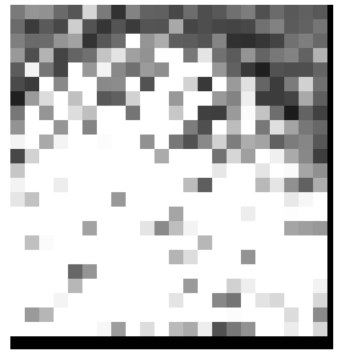

(h)

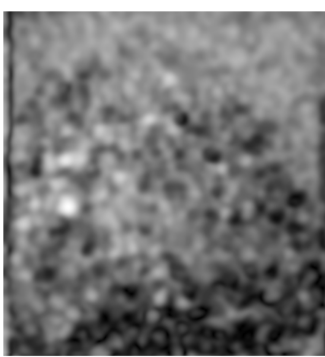

(1)

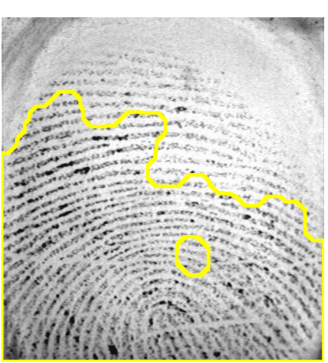

(e) $\mathrm{Err}=21.22$

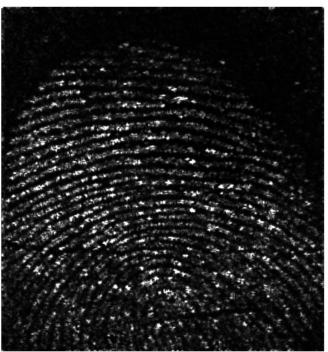

(i)

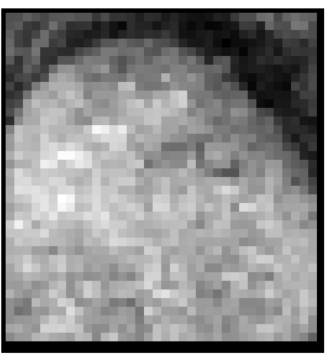

(m)

Figure 2.16: Segmented fingerprint images and their features of different methods for FVC2000_DB3_IM_17_3. (a) ground truth; (b, g) FDB, (c, h) Gabor, (d, i) Harris, (e, j, k, l) Mean-Variance-Coherence, (f, m) STFT. 


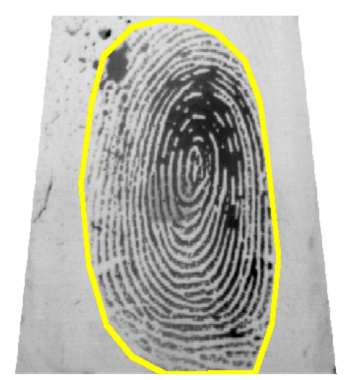

(a)

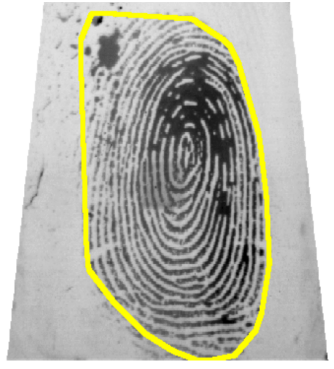

(b) $\mathrm{Err}=5.24$

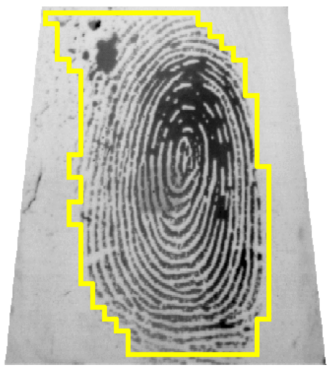

(f) $\operatorname{Err}=5.62$

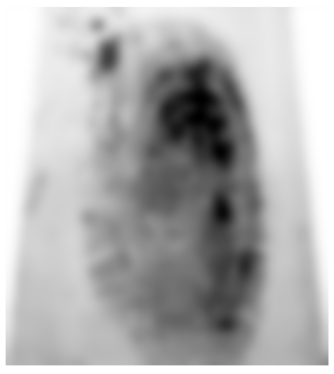

(j)

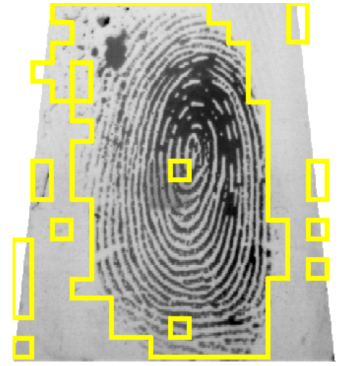

(c) $\operatorname{Err}=13.83$

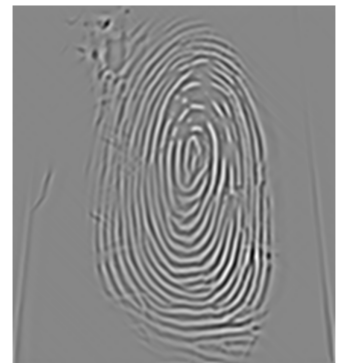

$(\mathrm{g})$

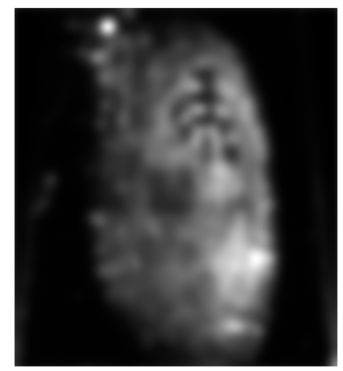

(k)

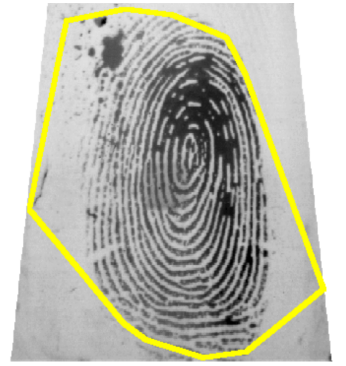

(d) $\operatorname{Err}=15.57$

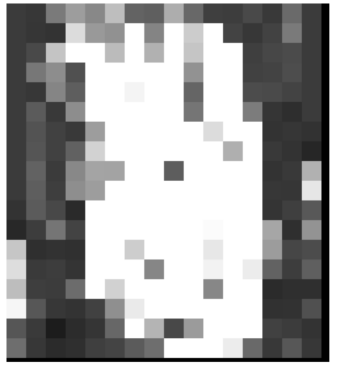

(h)

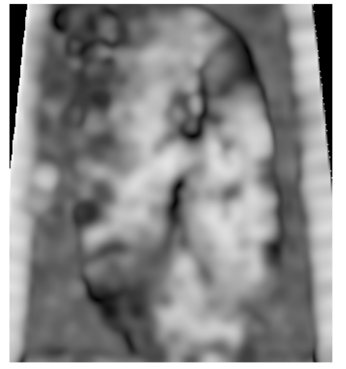

(1)

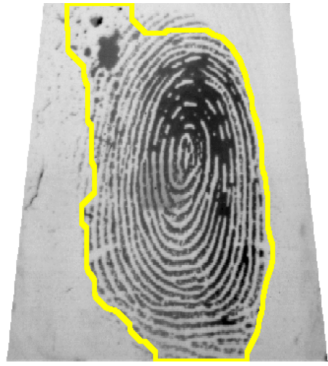

(e) $\operatorname{Err}=6.74$

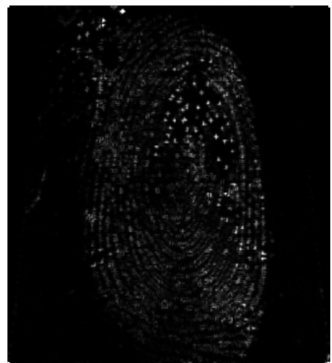

(i)

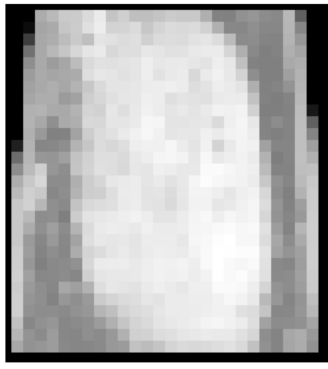

(m)

Figure 2.17: Segmented fingerprint images and their features of different methods for FVC2004_DB2_IM_56_8. (a) ground truth; (b, g) FDB, (c, h) Gabor, (d, i) Harris, (e, j, k, l) Mean-Variance-Coherence, (f, m) STFT. 


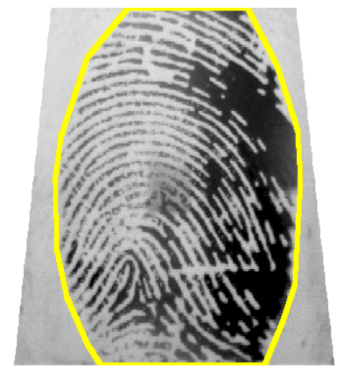

(a)

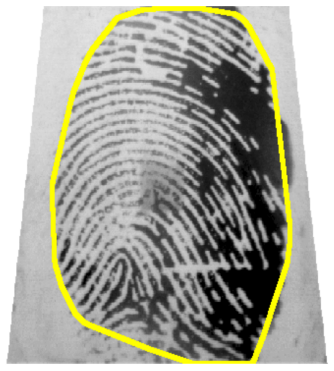

(b) $\operatorname{Err}=5.51$

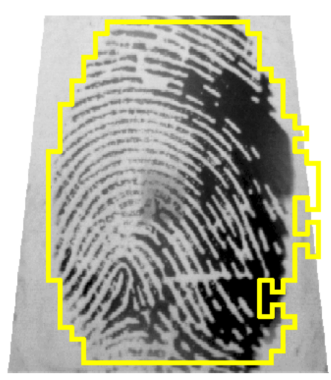

(f) $\operatorname{Err}=6.42$

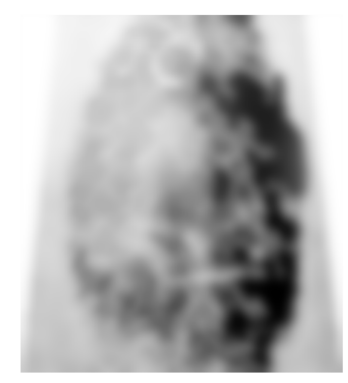

(j)

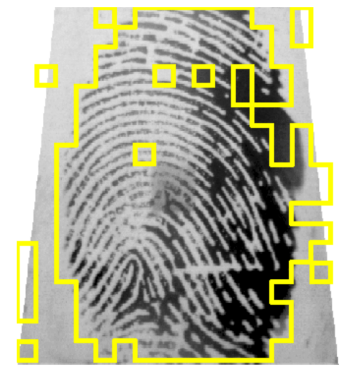

(c) $\operatorname{Err}=12.43$

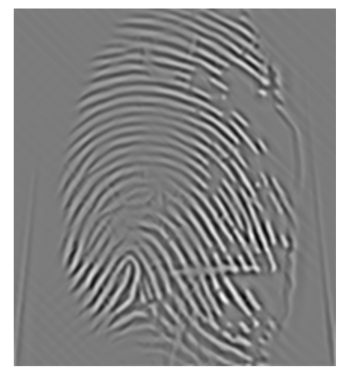

(g)

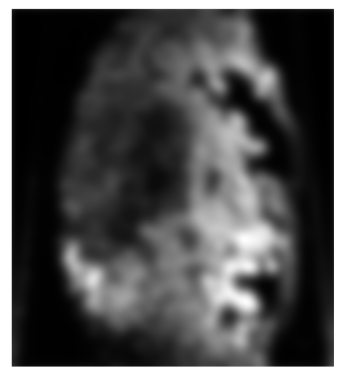

(k)

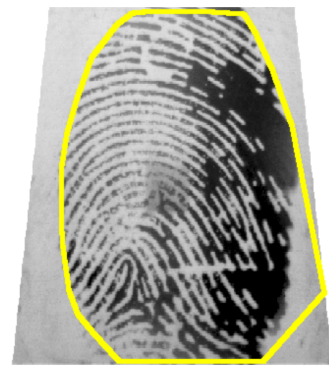

(d) $\mathrm{Err}=6.46$

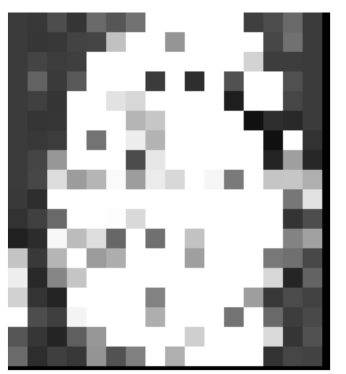

(h)

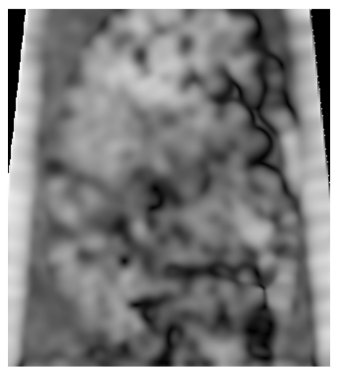

(1)

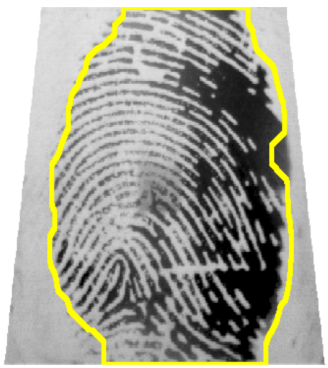

(e) $\operatorname{Err}=4.47$

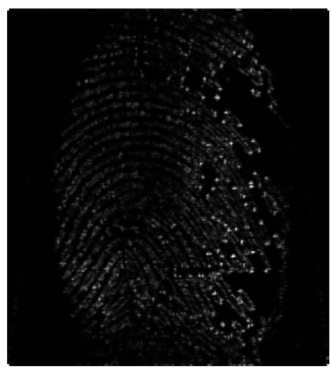

(i)

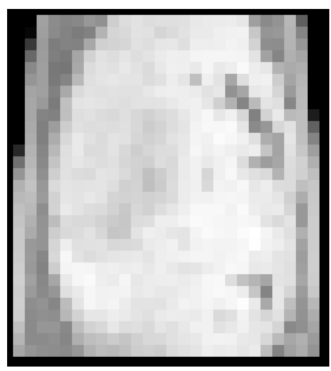

(m)

Figure 2.18: Segmented fingerprint images and their features of different methods for FVC2004_DB2_IM_65_7. (a) ground truth; (b, g) FDB, (c, h) Gabor, (d, i) Harris, (e, j, k, l) Mean-Variance-Coherence, (f, m) STFT. 


\section{Global Variational Method for Fingerprint Segmentation By Three-Part Decomposition}

\subsection{Introduction}

On the analogy to Chapter 2 (or [1]) with the Fourier based approach, in this chapter we design a variational based method to segment the fingerprint image into the foreground and the background.

\subsubsection{Global three-part decomposition (G3PD) method}

Our proposed method is based on the philosophy that a fingerprint image can be considered as a summation of three components: texture, homogeneous parts and small scale objects. In this chapter, we propose a global three-part decomposition (G3PD) method which aims to decompose a fingerprint image into the corresponding three parts:

- Texture image: By texture we refer to the fact that fingerprint images are highly determined by their oriented patterns which have frequencies only in a specific band in the Fourier spectrum, see [1].

- Cartoon image: The homogeneous regions correspond to the lower frequency response.

- Noise image: Small scale objects staying in the higher frequency band are considered as noise, e.g. black dots with random position and intensity.

For the purpose of fingerprint segmentation, we are only interested in the texture image as a feature for segmentation. After the decomposition, the cartoon and noise images are ignored.

Therefore, the decomposition can be considered as a feature extraction step which has the goal to obtain the best possible texture image from an input image. Subsequently, the texture image is binarized with a global threshold of 0 and morphological operations are adopted for attaining the region of interest (ROI), see Figure 3.1. In order to achieve these goals, we propose a model for three-part decomposition with variational based methods as described below. This variational approach follows the same philosophy of texture image extraction, but in a different view as the Fourier based FDB method [1]. 


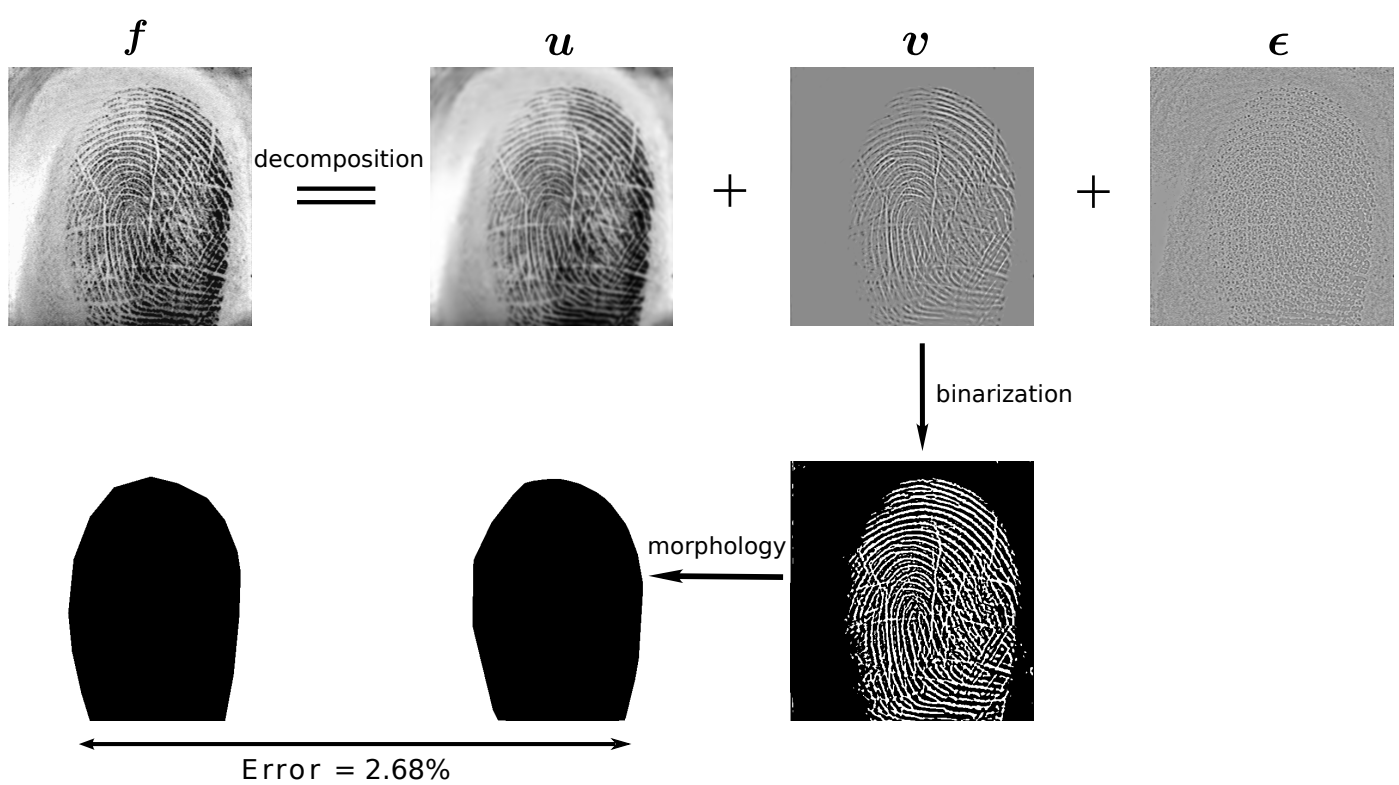

Figure 3.1: Overview over the segmentation by the variational method: Firstly, the original image (first row, left) is decomposed into the homogeneous regions (second column), texture (third column) and noise (fourth column). Secondly, the texture image is binarized with a threshold $T=0$ and the ROI is obtained by morphological operations. In order to evaluate the segmentation performance, the estimated ROI (second row, second column) is compared to manually marked ground truth segmentation (second row, first column). The chosen parameters of the decomposition is based on the training sets to avoid the halo effect on the texture image which causes the error for segmentation. Thus, the homogeneous regions and noise can contain some textures, but the result gives a better performance in the sense of image segmentation.

Proposed variational model for G3PD Decomposition techniques are at the core of variational methods. Decomposition is performed by finding the solution of a convex minimization problem. Inspired by this idea, we propose the TV $-G_{1,1}^{3 / 4}-\ell_{1}-G_{\infty, \infty}^{-3 / 4}$ model for global three-part decomposition. Piecewise constant regions are measured by the total variation (TV) norm. The sparsity of texture pattern is measured by the $\ell_{1}$ norm which relates to the shrinkage operator. The smoothness of texture image can be carried out by $G_{1,1}^{3 / 4}$ norms which corresponds to $\ell_{1}$ norm of curvelet coefficients [62]. The dual space of $G_{1,1}^{3 / 4}$ is $G_{\infty, \infty}^{-3 / 4}$ which relates to the small curvelet coefficients. This space is very suitable to capture the noise. By experiment, we have discovered that the curvelet shrinkage captures the geometry of fingerprint pattern better than the classical wavelet thresholding in terms of over-estimation and smoothness.

The combination of the decomposition and morphology in our proposed G3PD method yields segmentation performance superior to existing segmentation methods.

Performance Evaluation and Comparison to Existing Methods We conduct a systematic performance comparison of our proposed G3PD method with five state-of-the- 
art fingerprint segmentation methods. The segmentation accuracy of all methods is measured on a manually marked ground truth database containing 10560 images [1]. A detailed description of the evaluation benchmark, training and test protocols and experimental results is given in Section 3.3.

The five methods in the comparison are: a method based on mean and variance of gray level intensities and the coherence of gradients as features and a neural network as a classifier [7], a method using Gabor filter bank responses [8], a Harris corner response based method [9], an approach using local Fourier analysis [10] and the factorized directional bandpass method [1].

\subsubsection{Related Work}

With more than hundred methods, we refer the reader to [1] for an overview over the literature of fingerprint segmentation methods. For image segmentation in general, there is a plethora of approaches to solve this problem. These are based e.g. on the intensity of pixels $[63,64,65]$, or the evolution of curves for piecewise smooth regions in images [66, 67, 68, 69]. Texture segmentation, however, is still an open problem, because intensity values are inadequate, e.g. for segmenting fingerprint patterns. Methods based on texture descriptors [70,71] or finding other meaningful features in an observed image for classification have been suggested.

Based on the classical Rudin-Osher-Fatemi (ROF) model [72], researchers have proposed numerous approaches in which the regularization and fidelity terms are considered under different functional spaces, such as Besov, Hilbert and Banach spaces $[73,74,75,76,77,78]$, as well as higher order derivatives instead of the gradient in $\ell_{1}$ norm of the fidelity term, such as high-order PDEs [79, 80], mean curvature [81], and Euler's elastica [82]. Especially, many signals have sparse or nearly-sparse representations in some transform domain corresponding to $\ell_{0}$ or its regularization $\ell_{1}$ $[83,84,85,86,87]$. To solve the constrained minimization problems, various techniques have been suggested such as Chambolle's projection [88], splitting Bregman method [89], iterative shrinkage/thresholding (IST) algorithms [90, 91, 92]. Wu et al. [93] has proved the equivalence between augmented Lagrangian method (ALM), dual methods, and split Bregman iteration. We have adopted ALM into our approach to solve the proposed constrained minimization problem. [50], [94] and [95] show that the shrinkage operator of multiresolution analysis is the solution of a variational problem when considering signals in Besov space, i.e. $B_{p, q}^{\alpha}$, relating to wavelet coefficients. However, $B_{p, q}^{\alpha}$ is only suitable for signals with point-like features. The curvelet transform $[96,29,97,28]$, the contourlet transform [41], and steerable wavelet transform [98] have recently been introduced to capture the geometrical mechanism of images. These transforms are very suitable for image analysis, especially for fingerprint patterns with oriented and curved lines. In our framework, we perform a multiresolution analysis by the curvelet transform corresponding to an analysis of the signal under the curvelet type decomposition space, i.e. $G_{p, q}^{\alpha}$, which has been suggested by Borup and Nielsen [62].

There are many difficulties relating to the choices of the parameters for decomposition and minimization steps which ensure the convergence of the algorithm and extract enough texture for segmentation under the various situations, such as different illumi- 
nation, noise, and ghost fingerprints (see Figure 3.2 for an illustration). Addressing these problems is still a challenge in practice.

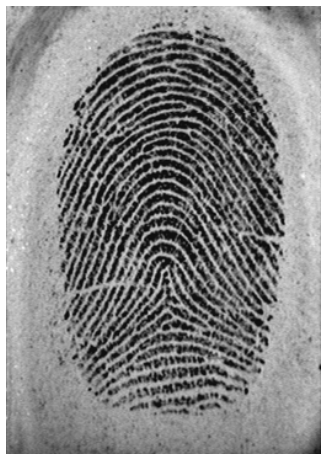

(a)

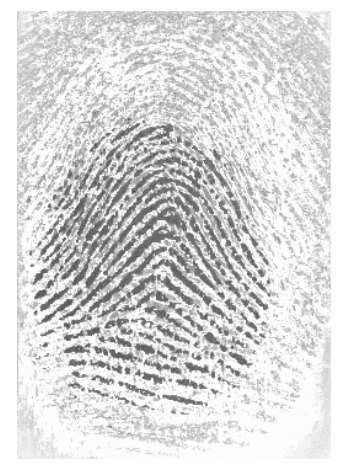

(b)

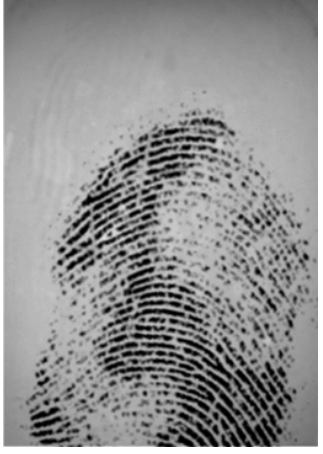

(c)

Figure 3.2: Difficult problems occur in images. (a) Small scale objects are modeled as noise. (b) Ghost fingerprint image. (c) Image with different illumination.

\subsubsection{Setup of Chapter 3}

The organization of this chapter is as follows:

In Section 3.2, we give a detailed description of the G3PD method in two main steps: first, texture image extraction is treated in Section 3.2.1, followed by morphological operations in Section 3.2.2, see Figure 3.1. To this end, we introduce the $\mathrm{TV}-G_{1,1}^{3 / 4}-\ell_{1}-G_{\infty, \infty}^{-3 / 4}$ model in Section 3.2.1.1 which defines the objective function as a constraint minimization problem for the decomposition of an image into three parts: cartoon, texture and noise images. In Section 3.2.1.2 we prove the existence and we discuss the uniqueness of the solution for the corresponding unconstraint minimization problem. Next, in Section 3.2.1.3 we apply the augmented Langrangian method to reformulate the constraint minimization into an unconstraint one. Subsequently, this unconstraint minimization problem is solved by the alternating direction method of multipliers (ADMM) in Section 3.2.1.4. The smoothness and sparsity of the obtained texture image as a feature for segmentation is discussed in Section 3.2.1.5. In Section 3.2 .2 , we specify how to obtain the ROI from the texture image by morphological operations. In Section 3.3 we describe the evaluation benchmark, the training and test protocols and experimental results. Finally, in Section 3.4 we discuss the results of the evaluation and we give conclusions. Notation, proofs and additional figures can be found in the Appendix.

\subsection{The G3PD Method for Fingerprint Segmentation}

This section describes the G3PD method which consists of two main parts: in the following Subsection 3.2.1, we introduce the TV $-G_{1,1}^{3 / 4}-\ell_{1}-G_{\infty, \infty}^{-3 / 4}$ model for three- 
part decomposition into cartoon, texture and noise images. Next, we formalize the constraint minimization problem and we discuss the ALM for solving it.

In Subsection 3.2.2, we utilize the obtained texture image as our feature to perform the segmentation by morphological operations.

\subsubsection{Fingerprint Texture Extraction}

\subsubsection{The $T V-G_{1,1}^{3 / 4}-\ell_{1}-G_{\infty, \infty}^{-3 / 4}$ Model}

As argued before, the fingerprint $\boldsymbol{f}$ is consists of the homogeneous region $\boldsymbol{u}$, the repeated patterns $\boldsymbol{v}$ staying in a frequency range in Fourier domain and corrupted by a certain random noise $\boldsymbol{\epsilon}$. Fundamental for our analysis is that we assume that the fingerprint pattern is sparse in the Fourier domain as the ridge lines form an oscillating signal at essentially one frequency, locally. We assume that

$$
f[\boldsymbol{k}]=u[\boldsymbol{k}]+v[\boldsymbol{k}]+\epsilon[\boldsymbol{k}], \quad \boldsymbol{k} \in \Omega_{d} .
$$

As laid out in Appendix 3.5, the space $B_{1,1}^{1}$ relating to the $\ell_{1}$ norm of the wavelet coefficients, i.e. $\|\boldsymbol{v}\|_{B_{1,1}^{1}}=\|\mathcal{W}\{\boldsymbol{v}\}\|_{\ell_{1}}$, is very suitable to measure the smoothness of the oscillation signals. However, due to a set of highly curved lines in the fingerprint patterns, the space $G_{1,1}^{3 / 4}$ is considered instead to capture their curvature, called texture $\boldsymbol{v}$. Denoting $\mathcal{C}\{\boldsymbol{v}\}=\left[\mathcal{C}_{i, l}\{\boldsymbol{v}\}\right]_{(i, l) \in \mathcal{I}}$ the curvelet transform of $\boldsymbol{v}$ in $i$ different scales and $l$ orientations. The norm of $\boldsymbol{v}$ in the space $G_{1,1}^{3 / 4}$ is $\|\boldsymbol{v}\|_{G_{1,1}^{3 / 4}}=\|\mathcal{C}\{\boldsymbol{v}\}\|_{\ell_{1}}$. In order to get the sparse texture $\boldsymbol{v}$ in the spatial domain, the $\ell_{1}$ norm is adopted. In conclusion, the norms $\left\{\|\mathcal{C}\{\boldsymbol{v}\}\|_{\ell_{1}}+\|\boldsymbol{v}\|_{\ell_{1}}\right\}$ are considered to extract the fingerprint patterns. Then, the bounded variation space with the discrete TV-norm, i.e. $J(\boldsymbol{u})=\left\|\nabla_{\mathrm{d}} \boldsymbol{u}\right\|_{\ell_{1}}\left(\nabla_{\mathrm{d}}\right.$ is the discrete gradient operator in Appendix 3.7.1), is well-known to measure the roughness of a piecewise constant image $\boldsymbol{u}$ [72]. Finally, the residual $\boldsymbol{\epsilon}$ is considered in $G_{\infty, \infty}^{-3 / 4}$, the dual space of $G_{1,1}^{3 / 4}$, relating to small curvelet coefficients, i.e.

$$
\|\boldsymbol{\epsilon}\|_{G_{\infty, \infty}^{-3 / 4}}:=\|\mathcal{C}\{\boldsymbol{\epsilon}\}\|_{\infty}=\sup _{i, l, \boldsymbol{k} \in \mathcal{I}}\left|\mathcal{C}_{i, l}\{\boldsymbol{\epsilon}\}[\boldsymbol{k}]\right| .
$$

Thus, the constraint of the minimization problem is defined: the norm of the residual in $G_{\infty, \infty}^{-3 / 4}$, i.e. $\|\mathcal{C}\{\boldsymbol{f}-\boldsymbol{u}-\boldsymbol{v}\}\|_{\infty}$, is less than some level of noise $\delta$. In summary, the variational model is described in the Euclidean space $X$ (cf. Appendix 3.5) as follows

$$
(\overline{\boldsymbol{u}}, \overline{\boldsymbol{v}})=\underset{(\boldsymbol{u}, \boldsymbol{v}) \in X^{2}}{\operatorname{argmin}}\{\left\|\nabla_{\mathrm{d}} \boldsymbol{u}\right\|_{\ell_{1}}+\mu_{1}\|\mathcal{C}\{\boldsymbol{v}\}\|_{\ell_{1}}+\mu_{2}\|\boldsymbol{v}\|_{\ell_{1}} \text { s.t. } \underbrace{\sup _{i, l, \boldsymbol{k} \in \mathcal{I}}\left|\mathcal{C}_{i, l}\{\boldsymbol{f}-\boldsymbol{u}-\boldsymbol{v}\}[\boldsymbol{k}]\right|}_{=\|\mathcal{C}\{\boldsymbol{f}-\boldsymbol{u}-\boldsymbol{v}\}\|_{\infty}} \leq \delta\} .
$$

Note that the form of (3.1) is analogous to the statistical multiresolution estimator in [99] (or [100]) where the nonlinear transformation is the absolute value of the curvelet coefficients, i.e. $\Lambda(\cdot)=|\mathcal{C}\{\cdot\}|$, the length of subsets $|\mathcal{S}|=1$ and the weight-function $\omega^{\mathcal{S}}=1$. The main difference is that our model has two variables $(\boldsymbol{u}, \boldsymbol{v})$. With the 
residual $\boldsymbol{\epsilon}=\boldsymbol{f}-\boldsymbol{u}-\boldsymbol{v}$, the constraint minimization (3.1) is rewritten as

$$
(\overline{\boldsymbol{u}}, \overline{\boldsymbol{v}}, \overline{\boldsymbol{\epsilon}})=\underset{(\boldsymbol{u}, \boldsymbol{v}, \boldsymbol{\epsilon}) \in X^{3}}{\operatorname{argmin}}\left\{\left\|\nabla_{\mathrm{d}} \boldsymbol{u}\right\|_{\ell_{1}}+\mu_{1}\|\mathcal{C}\{\boldsymbol{v}\}\|_{\ell_{1}}+\mu_{2}\|\boldsymbol{v}\|_{\ell_{1}} \text { s.t. }\|\mathcal{C}\{\boldsymbol{\epsilon}\}\|_{\infty} \leq \delta, \boldsymbol{f}=\boldsymbol{u}+\boldsymbol{v}+\boldsymbol{\epsilon}\right\} .
$$

Given $\delta>0$, denote the indicator function on the feasible convex set $S(\delta)$ of $(3.2)$ by $G^{*}\left(\frac{\epsilon}{\delta}\right)$ (cf. Proposition 3.5.1), i.e.

$$
S(\delta)=\left\{\boldsymbol{\epsilon} \in X \mid\|\mathcal{C}\{\boldsymbol{\epsilon}\}\|_{\infty} \leq \delta\right\} \text { and } G^{*}\left(\begin{array}{l}
\boldsymbol{\epsilon} \\
\delta
\end{array}\right)= \begin{cases}0, & \boldsymbol{\epsilon} \in S(\delta) \\
+\infty, & \boldsymbol{\epsilon} \in X \backslash S(\delta) .\end{cases}
$$

By changing the inequality constraint into the indicator function $G^{*}\left(\frac{\epsilon}{\delta}\right),(3.2)$ is rewritten as a convex minimization of four convex functions and one equality constraint:

$(\overline{\boldsymbol{u}}, \overline{\boldsymbol{v}}, \overline{\boldsymbol{\epsilon}})=\underset{(\boldsymbol{u}, \boldsymbol{v}, \boldsymbol{\epsilon}) \in X^{3}}{\operatorname{argmin}}\left\{\left\|\nabla_{\mathrm{d}} \boldsymbol{u}\right\|_{\ell_{1}}+\mu_{1}\|\mathcal{C}\{\boldsymbol{v}\}\|_{\ell_{1}}+\mu_{2}\|\boldsymbol{v}\|_{\ell_{1}}+G^{*}\left(\frac{\boldsymbol{\epsilon}}{\delta}\right)\right.$ s.t. $\left.\boldsymbol{f}=\boldsymbol{u}+\boldsymbol{v}+\boldsymbol{\epsilon}\right\}$.

The original image $\boldsymbol{f}$ is therefore decomposed into the piecewise-constant image $\boldsymbol{u}$, the texture $\boldsymbol{v}$ and the small scale objects modeling as noise $\boldsymbol{\epsilon}$ by minimizing the objective function (3.3).

\subsubsection{Existence and Uniqueness of the corresponding unconstrained minimization of (3.3)}

As mentioned before, the original image $\boldsymbol{f}$ is decomposed into $\boldsymbol{u}, \boldsymbol{v}$ and $\boldsymbol{\epsilon}$ by minimizing (3.3). In this section, we describe how to turn the constrained minimization (3.3) into unconstrained one by the ALM in the first paragraph. Then, we discuss the existence and the uniqueness of solution for its corresponding unconstrained problem in the second paragraph by Proposition 3.2.1 and 3.2.2, respectively.

Unconstrained minimization of (3.3) by Augmented Lagrangian method We have a constrained minimization problem, and by analogy with Aujol et al. [101], the penalty method is applied to turn (3.3) into an unconstrained minimization problem:

$$
\begin{gathered}
\left(\overline{\boldsymbol{u}}_{\mathrm{pen}}, \overline{\boldsymbol{v}}_{\mathrm{pen}}, \overline{\boldsymbol{\epsilon}}_{\mathrm{pen}}\right)=\underset{(\boldsymbol{u}, \boldsymbol{v}, \boldsymbol{\epsilon}) \in X^{3}}{\operatorname{argmin}}\{ \\
\left\|\nabla_{\mathrm{d}} \boldsymbol{u}\right\|_{\ell_{1}}+\mu_{1}\|\mathcal{C}\{\boldsymbol{v}\}\|_{\ell_{1}}+\mu_{2}\|\boldsymbol{v}\|_{\ell_{1}}+G^{*}\left(\frac{\boldsymbol{\epsilon}}{\delta}\right) \\
\left.+\frac{\beta}{2}\|\boldsymbol{f}-\boldsymbol{u}-\boldsymbol{v}-\boldsymbol{\epsilon}\|_{\ell_{2}}^{2}\right\} .
\end{gathered}
$$

However, $\left(\overline{\boldsymbol{u}}_{\text {pen }}, \overline{\boldsymbol{v}}_{\text {pen }}, \overline{\boldsymbol{\epsilon}}_{\text {pen }}\right)$ is equivalent to $(\overline{\boldsymbol{u}}, \overline{\boldsymbol{v}}, \overline{\boldsymbol{\epsilon}})$ if and only if $\beta$ is infinity which causes difficulties for numerical computation. In order to overcome this, the augmented Lagrangian method with the Lagrange multiplier $\boldsymbol{\lambda} \in X$ and the regularization 
parameter $\beta \in(0,+\infty)$ is applied instead:

$$
\begin{aligned}
&\left(\overline{\boldsymbol{u}}_{\mathrm{ALM}}, \overline{\boldsymbol{v}}_{\mathrm{ALM}}, \overline{\boldsymbol{\epsilon}}_{\mathrm{ALM}}\right)=\underset{(\boldsymbol{u}, \boldsymbol{v}, \boldsymbol{\epsilon}) \in X^{3}}{\operatorname{argmin}}\{\left\|\nabla_{\mathrm{d}} \boldsymbol{u}\right\|_{\ell_{1}}+\mu_{1}\|\mathcal{C}\{\boldsymbol{v}\}\|_{\ell_{1}}+\mu_{2}\|\boldsymbol{v}\|_{\ell_{1}}+G^{*}\left(\frac{\boldsymbol{\epsilon}}{\delta}\right) \\
&\left.+\langle\boldsymbol{\lambda}, \boldsymbol{f}-\boldsymbol{u}-\boldsymbol{v}-\boldsymbol{\epsilon}\rangle_{\ell_{2}}+\frac{\beta}{2}\|\boldsymbol{f}-\boldsymbol{u}-\boldsymbol{v}-\boldsymbol{\epsilon}\|_{\ell_{2}}^{2}\right\},
\end{aligned}
$$

which is rewritten as

$$
\left(\overline{\boldsymbol{u}}_{\mathrm{ALM}}, \overline{\boldsymbol{v}}_{\mathrm{ALM}}, \overline{\boldsymbol{\epsilon}}_{\mathrm{ALM}}\right):=\underset{(\boldsymbol{u}, \boldsymbol{v}, \boldsymbol{\epsilon}) \in X^{3}}{\operatorname{argmin}} \mathcal{L}_{\beta}(\boldsymbol{u}, \boldsymbol{v}, \boldsymbol{\epsilon} ; \boldsymbol{\lambda})
$$

and

$$
\mathcal{L}_{\beta}(\boldsymbol{u}, \boldsymbol{v}, \boldsymbol{\epsilon} ; \boldsymbol{\lambda})=\left\|\nabla_{\mathrm{d}} \boldsymbol{u}\right\|_{\ell_{1}}+\mu_{1}\|\mathcal{C}\{\boldsymbol{v}\}\|_{\ell_{1}}+\mu_{2}\|\boldsymbol{v}\|_{\ell_{1}}+G^{*}\left(\frac{\boldsymbol{\epsilon}}{\delta}\right)+\frac{\beta}{2}\left\|\boldsymbol{f}-\boldsymbol{u}-\boldsymbol{v}-\boldsymbol{\epsilon}+\frac{\boldsymbol{\lambda}}{\beta}\right\|_{\ell_{2}}^{2} .
$$

Numerically, the minimizer of (3.4) is obtained through the number of iterations $(n=$ $1,2, \ldots)$ with the updated Lagrange multiplier $\boldsymbol{\lambda}^{(n)}$ initialized at $\boldsymbol{\lambda}^{(0)}=0$ :

$$
\begin{array}{r}
\left(\boldsymbol{u}^{(n)}, \boldsymbol{v}^{(n)}, \boldsymbol{\epsilon}^{(n)}\right)=\underset{(\boldsymbol{u}, \boldsymbol{v}, \boldsymbol{\epsilon}) \in X^{3}}{\operatorname{argmin}} \mathcal{L}_{\beta}\left(\boldsymbol{u}, \boldsymbol{v}, \boldsymbol{\epsilon} ; \boldsymbol{\lambda}^{(n-1)}\right), \\
\boldsymbol{\lambda}^{(n)}=\boldsymbol{\lambda}^{(n-1)}+\beta\left(\boldsymbol{f}-\boldsymbol{u}^{(n)}-\boldsymbol{v}^{(n)}-\boldsymbol{\epsilon}^{(n)}\right) .
\end{array}
$$

If the number of iterations $n$ goes to infinity, then the solution of (3.4) is equivalent to the solution of (3.2) (or (3.3)):

$$
\left(\boldsymbol{u}^{(\infty)}, \boldsymbol{v}^{(\infty)}, \boldsymbol{\epsilon}^{(\infty)}\right) \equiv\left(\overline{\boldsymbol{u}}_{\mathrm{ALM}}, \overline{\boldsymbol{v}}_{\mathrm{ALM}}, \overline{\boldsymbol{\epsilon}}_{\mathrm{ALM}}\right) \equiv(\overline{\boldsymbol{u}}, \overline{\boldsymbol{v}}, \overline{\boldsymbol{\epsilon}})
$$

Due to the appearance of $\boldsymbol{\lambda}, \beta$ can be much smaller than the infinity which avoids the ill-possedness at infinity. In other words, the role of Lagrange multiplier $\boldsymbol{\lambda}$ is to compensate for $\beta \neq \infty$.

Discussion of the existence and uniqueness of (3.4) Similarly to [74], [101] and [102] for the penalty method, in this section we discuss the existence and the uniqueness of the minimization (3.4). Due to the compensation of the updated Lagrange multiplier $\lambda^{(n)}$ for $\beta \neq \infty$ and the convergence of solution when $n$ goes to infinity, $\lambda^{(n)}$ can be considered as an updated constant. Therefore, we can say that the existence of a saddle point of $\mathcal{L}_{\beta}$ with the updated $\boldsymbol{\lambda}$, i.e.

$$
\mathcal{L}_{\beta}(\overline{\boldsymbol{u}}, \overline{\boldsymbol{v}}, \overline{\boldsymbol{\epsilon}} ; \boldsymbol{\lambda}) \leq \mathcal{L}_{\beta}(\overline{\boldsymbol{u}}, \overline{\boldsymbol{v}}, \overline{\boldsymbol{\epsilon}} ; \overline{\boldsymbol{\lambda}}) \leq \mathcal{L}_{\beta}(\boldsymbol{u}, \boldsymbol{v}, \boldsymbol{\epsilon} ; \overline{\boldsymbol{\lambda}}), \forall(\boldsymbol{u}, \boldsymbol{v}, \boldsymbol{\epsilon}, \boldsymbol{\lambda}) \in X^{4},
$$

follows from the existence of solution of $\mathcal{L}_{\beta}$ with considering $\boldsymbol{\lambda}$ as a constant in (3.4).

Proposition 3.2.1. For the minimization problem (3.4), there exists a minimizer $(\overline{\boldsymbol{u}}, \overline{\boldsymbol{v}}, \overline{\boldsymbol{\epsilon}})$.

Proof. For simplicity of the notation, we call the objective function $H(\boldsymbol{u}, \boldsymbol{v}, \boldsymbol{\epsilon})=$ $\mathcal{L}_{\beta}(\boldsymbol{u}, \boldsymbol{v}, \boldsymbol{\epsilon} ; \boldsymbol{\lambda})$ with $\beta \neq \infty$ and the updated constant $\boldsymbol{\lambda}$. In order to prove the existence of a minimizer, we have to show that $H(\boldsymbol{u}, \boldsymbol{v}, \boldsymbol{\epsilon})$ is convex, lower semi continuous and coercive. 
- The domain of $\boldsymbol{u}$ and $\boldsymbol{v}$ are $\boldsymbol{u} \in X, \boldsymbol{v} \in X$, respectively. If $\boldsymbol{\epsilon} \in S(\delta)$, then the indicator function $G^{*}\left(\frac{\epsilon}{\delta}\right)$ is 0 , otherwise, i.e. $\epsilon \in X \backslash S(\delta), G^{*}\left(\frac{\epsilon}{\delta}\right)$ is infinity.

- Convexity of $H(\boldsymbol{u}, \boldsymbol{v}, \boldsymbol{\epsilon})$ :

Obviously, the domain $X \times X \times S(\delta)$ is convex. If $\boldsymbol{\epsilon} \in S(\delta)$, then $H(\boldsymbol{u}, \boldsymbol{v}, \boldsymbol{\epsilon})$ is convex on $X \times X \times S(\delta)$, because the sum of convex functions is convex. If $\boldsymbol{\epsilon} \in X \backslash S(\delta)$, then $H(\boldsymbol{u}, \boldsymbol{v}, \boldsymbol{\epsilon})=+\infty$. In conclusion, $H(\boldsymbol{u}, \boldsymbol{v}, \boldsymbol{\epsilon})$ is convex on $X \times X \times X$.

- Lower semi continuity:

If $\boldsymbol{\epsilon} \in S(\delta)$, then $H(\boldsymbol{u}, \boldsymbol{v}, \boldsymbol{\epsilon})$ is continuous on $X \times X \times S(\delta)$; otherwise, i.e. $\boldsymbol{\epsilon} \in$ $X \backslash S(\delta), H(\boldsymbol{u}, \boldsymbol{v}, \boldsymbol{\epsilon})=+\infty$. Thus, $H(\boldsymbol{u}, \boldsymbol{v}, \boldsymbol{\epsilon})$ is lower semi continuous on $X \times X \times$ $X$.

- $f(x)$ is coercive, i.e. $\lim _{\|x\| \rightarrow+\infty} f(x) \rightarrow+\infty$ :

In order to prove this, we have to prove that

$\lim _{\|(\boldsymbol{u}, \boldsymbol{v}, \boldsymbol{\epsilon})\|_{X^{3}} \rightarrow+\infty} H(\boldsymbol{u}, \boldsymbol{v}, \boldsymbol{\epsilon}) \rightarrow+\infty \quad$ with $\|(\boldsymbol{u}, \boldsymbol{v}, \boldsymbol{\epsilon})\|_{X^{3}}=\sqrt{\|\boldsymbol{u}\|_{X}^{2}+\|\boldsymbol{v}\|_{X}^{2}+\|\boldsymbol{\epsilon}\|_{X}^{2}}$.

If $\|\boldsymbol{\epsilon}\|_{X} \rightarrow+\infty$ as $\|(\boldsymbol{u}, \boldsymbol{v}, \boldsymbol{\epsilon})\|_{X^{3}} \rightarrow+\infty$, then $\boldsymbol{\epsilon} \in X \backslash S(\delta)$ and $H(\boldsymbol{u}, \boldsymbol{v}, \boldsymbol{\epsilon})=+\infty$, i.e.

$$
\lim _{\|(\boldsymbol{u}, \boldsymbol{v}, \boldsymbol{\epsilon})\|_{X^{3}} \rightarrow+\infty} H(\boldsymbol{u}, \boldsymbol{v}, \boldsymbol{\epsilon}) \rightarrow+\infty .
$$

If $\boldsymbol{\epsilon}$ remains in $S(\delta)$, then $\|\boldsymbol{\epsilon}\|_{G_{\infty, \infty}^{-3 / 4}} \leq \delta$ and

$$
\exists c>0 \text { s.t. }\|\boldsymbol{\epsilon}\|_{X} \leq c\|\boldsymbol{\epsilon}\|_{G_{\infty, \infty}^{-3 / 4}} \leq c \delta .
$$

Thus,

$$
\|(\boldsymbol{u}, \boldsymbol{v}, \boldsymbol{\epsilon})\|_{X^{3}} \rightarrow+\infty \quad \text { i.f.f. } \quad\|\boldsymbol{u}\|_{X}^{2}+\|\boldsymbol{v}\|_{X}^{2} \rightarrow+\infty
$$

Since $H(\boldsymbol{u}, \boldsymbol{v}, \boldsymbol{\epsilon}) \geq \frac{\beta}{2}\left\|\boldsymbol{f}-\boldsymbol{u}-\boldsymbol{v}-\boldsymbol{\epsilon}+\frac{\boldsymbol{\lambda}}{\beta}\right\|_{\ell_{2}}^{2} \rightarrow+\infty$, we have $H(\boldsymbol{u}, \boldsymbol{v}, \boldsymbol{\epsilon}) \rightarrow+\infty$.

Thus, $H(\boldsymbol{u}, \boldsymbol{v}, \boldsymbol{\epsilon})$ is coercive.

Proposition 3.2.2. A minimizer $(\overline{\boldsymbol{u}}, \overline{\boldsymbol{v}}, \overline{\boldsymbol{\epsilon}})$ is not necessarily unique, but $(\overline{\boldsymbol{u}}+\overline{\boldsymbol{v}}+\overline{\boldsymbol{\epsilon}})$ is unique.

Proof. The objective function (3.4) is strictly convex as a sum of four convex functions

$$
(\underbrace{\left\|\nabla_{\mathrm{d}} \boldsymbol{u}\right\|_{\ell_{1}}}_{:=J(\boldsymbol{u})}+\mu_{1}\|\mathcal{C}\{\boldsymbol{v}\}\|_{\ell_{1}}+\mu_{2}\|\boldsymbol{v}\|_{\ell_{1}}+G^{*}\left(\frac{\boldsymbol{\epsilon}}{\delta}\right))
$$

and a strictly convex function $\left\|\boldsymbol{f}-\boldsymbol{u}-\boldsymbol{v}-\boldsymbol{\epsilon}+\frac{\boldsymbol{\lambda}}{\beta}\right\|_{\ell_{2}}^{2}$, except in the directions $\left(\boldsymbol{w}_{1}+\right.$ $\left.\boldsymbol{w}_{2},-\boldsymbol{w}_{1},-\boldsymbol{w}_{2}\right)$ for any $\boldsymbol{w}_{1}, \boldsymbol{w}_{\mathbf{2}} \in X$. Since $J(\boldsymbol{u})$ is only convex, not strictly convex, 
we obtain that if $\left(\overline{\boldsymbol{u}}_{\mathbf{1}}, \overline{\boldsymbol{v}}_{\mathbf{1}}, \overline{\boldsymbol{\epsilon}}_{\mathbf{1}}\right)$ and $\left(\overline{\boldsymbol{u}}_{\mathbf{2}}, \overline{\boldsymbol{v}}_{\mathbf{2}}, \overline{\boldsymbol{\epsilon}}_{\mathbf{2}}\right)$ are minimizers, then $\overline{\boldsymbol{u}}_{\mathbf{1}}+\overline{\boldsymbol{v}}_{\mathbf{1}}+\overline{\boldsymbol{\epsilon}}_{\mathbf{1}}=$ $\overline{\boldsymbol{u}}_{\mathbf{2}}+\overline{\boldsymbol{v}}_{\mathbf{2}}+\overline{\boldsymbol{\epsilon}}_{\mathbf{2}}$. Therefore, if $(\overline{\boldsymbol{u}}, \overline{\boldsymbol{v}}, \overline{\boldsymbol{\epsilon}})$ is a minimizer, then $(\overline{\boldsymbol{u}}+\overline{\boldsymbol{v}}+\overline{\boldsymbol{\epsilon}})$ is unique.

Since (3.4) is only convex (and not strictly convex), its solution exists but is not unique (cf. Proposition 3.2.1 and 3.2.2). Minimization (3.4) is solved by iteration with the initial variables. Given different initializations, the minimization (3.4) has different minimizers $(u, v, \epsilon)$. Analyzing which minimizer is more suitable is beyond the scope of this chapter. Hence, we initialize for all variables as 0 , as it is described later in Algorithm 1.

\subsubsection{Augmented Lagrangian Method to Reformulate the Constrained Minimization Problem in Equation (3.4)}

There are different kinds of norms in (3.4). In order to make a simple calculation, we introduce new variables

$$
\left\{\begin{array}{l}
\boldsymbol{p}=\nabla_{\mathrm{d}} \boldsymbol{u}=\left[\boldsymbol{p}_{1}, \boldsymbol{p}_{2}\right]^{T}, \\
\boldsymbol{w}=\left[\boldsymbol{w}_{i, l}\right]_{(i, l) \in \mathcal{I}}=\mathcal{C}\{\boldsymbol{v}\},
\end{array}\right.
$$

and rename $\beta_{3}=\beta$ and $\boldsymbol{\lambda}_{\mathbf{3}}=\boldsymbol{\lambda}$ for convenient computation.

Then, (3.4) becomes a constrained minimization. Again, we apply the ALM. The augmented Lagrangian function of (3.4) with the three Lagrange multipliers $\left(\boldsymbol{\lambda}_{\mathbf{1}}, \boldsymbol{\lambda}_{\mathbf{2}}, \boldsymbol{\lambda}_{\mathbf{3}}\right)$ is defined as

$$
\left(\boldsymbol{u}^{*}, \boldsymbol{v}^{*}, \boldsymbol{\epsilon}^{*}, \boldsymbol{w}^{*}, \boldsymbol{p}^{*}\right)=\underset{\boldsymbol{u}, \boldsymbol{v}, \boldsymbol{\epsilon}, \boldsymbol{w}, \boldsymbol{p} \in X^{3} \times \mathbb{R}|\mathcal{I}| \times Y}{\operatorname{argmin}} \mathcal{L}\left(\boldsymbol{u}, \boldsymbol{v}, \boldsymbol{\epsilon}, \boldsymbol{w}, \boldsymbol{p} ; \boldsymbol{\lambda}_{\mathbf{1}}, \boldsymbol{\lambda}_{\mathbf{2}}, \boldsymbol{\lambda}_{\mathbf{3}}\right),
$$

where

$$
\begin{aligned}
\mathcal{L}\left(\boldsymbol{u}, \boldsymbol{v}, \boldsymbol{\epsilon}, \boldsymbol{w}, \boldsymbol{p} ; \boldsymbol{\lambda}_{\mathbf{1}}, \boldsymbol{\lambda}_{\mathbf{2}}, \boldsymbol{\lambda}_{\mathbf{3}}\right)=\|\boldsymbol{p}\|_{\ell_{1}}+\mu_{1}\|\boldsymbol{w}\|_{\ell_{1}}+\mu_{2}\|\boldsymbol{v}\|_{\ell_{1}}+G^{*}\left(\frac{\boldsymbol{\epsilon}}{\delta}\right) \\
\quad+\frac{\beta_{1}}{2}\left\|\boldsymbol{p}-\nabla_{\mathrm{d}} \boldsymbol{u}+\frac{\boldsymbol{\lambda}_{\mathbf{1}}}{\beta_{1}}\right\|_{\ell_{2}}^{2}+\frac{\beta_{2}}{2}\left\|\boldsymbol{w}-\mathcal{C}\{\boldsymbol{v}\}+\frac{\boldsymbol{\lambda}_{\mathbf{2}}}{\beta_{2}}\right\|_{\ell_{2}}^{2}+\frac{\beta_{3}}{2}\left\|\boldsymbol{f}-\boldsymbol{u}-\boldsymbol{v}-\boldsymbol{\epsilon}+\frac{\boldsymbol{\lambda}_{\mathbf{3}}}{\beta_{3}}\right\|_{\ell_{2}}^{2} .
\end{aligned}
$$

The minimizer of (3.5) is numerically computed through iterations $n=1,2, \ldots$

$$
\left(\boldsymbol{u}^{(n)}, \boldsymbol{v}^{(n)}, \boldsymbol{\epsilon}^{(n)}, \boldsymbol{w}^{(n)}, \boldsymbol{p}^{(n)}\right)=\underset{\boldsymbol{u}, \boldsymbol{v}, \boldsymbol{\epsilon}, \boldsymbol{w}, \boldsymbol{p} \in X^{3} \times \mathbb{R}|\mathcal{I}| \times Y}{\operatorname{argmin}} \mathcal{L}\left(\boldsymbol{u}, \boldsymbol{v}, \boldsymbol{\epsilon}, \boldsymbol{w}, \boldsymbol{p} ; \boldsymbol{\lambda}_{\mathbf{1}}^{(n-1)}, \boldsymbol{\lambda}_{\mathbf{2}}^{(n-1)}, \boldsymbol{\lambda}_{\mathbf{3}}^{(n-1)}\right)
$$

and the Lagrange multipliers are updated after every step $n$ with a rate $\gamma$ and the initial $\boldsymbol{\lambda}_{1}^{(0)}=\boldsymbol{\lambda}_{2}^{(0)}=\boldsymbol{\lambda}_{3}^{(0)}=0$ :

$$
\left\{\begin{array}{l}
\boldsymbol{\lambda}_{\mathbf{1}}^{(n)}=\boldsymbol{\lambda}_{\mathbf{1}}^{(n-1)}+\gamma \beta_{1}\left(\boldsymbol{p}^{(n)}-\nabla_{\mathrm{d}} \boldsymbol{u}^{(n)}\right) \\
\boldsymbol{\lambda}_{\mathbf{2}}^{(n)}=\boldsymbol{\lambda}_{\mathbf{2}}^{(n-1)}+\gamma \beta_{2}\left(\boldsymbol{w}^{(n)}-\mathcal{C}\left\{\boldsymbol{v}^{(n)}\right\}\right) \\
\boldsymbol{\lambda}_{\mathbf{3}}^{(n)}=\boldsymbol{\lambda}_{\mathbf{3}}^{(n-1)}+\gamma \beta_{3}\left(\boldsymbol{f}-\boldsymbol{u}^{(n)}-\boldsymbol{v}^{(n)}-\boldsymbol{\epsilon}^{(n)}\right) .
\end{array}\right.
$$

When the number of iterations $n$ goes to infinity, the solutions to (3.3), (3.4), (3.5) and (3.6) are equivalent. However, due to the computational time in practice, we stop 
after a small number of iterations, i.e. the number of iterations $n$ is bounded by some $N \in \mathbb{N}$, and we obtain an approximate solution (cf. Algorithm 1).

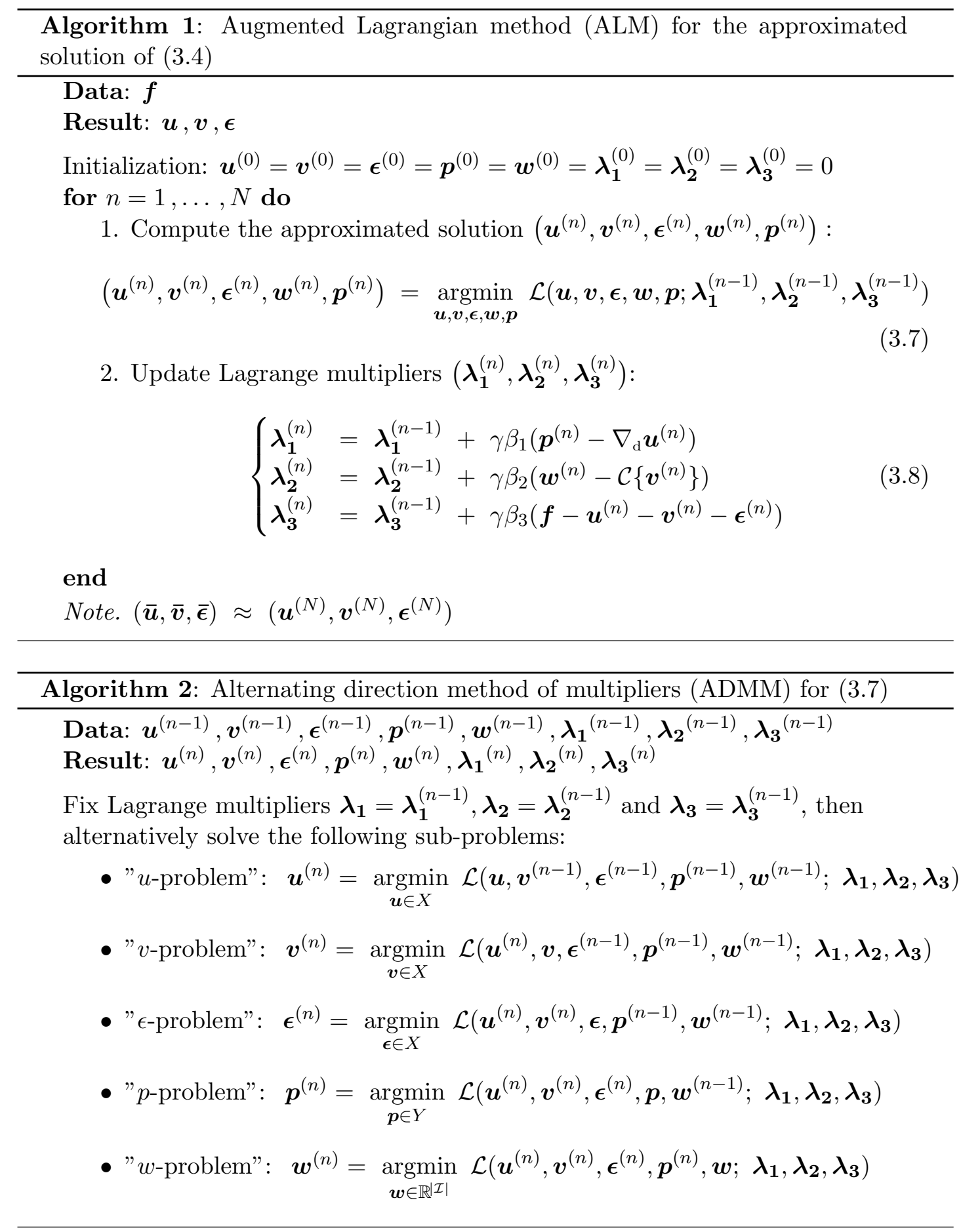

In the following part, we will describe the algorithm to solve the minimization problem (3.7) by the alternating direction method of multipliers (ADMM). 


\subsubsection{Alternating direction method of multipliers and numerical implementation}

Similarly to $[82,80,93,103,79,81]$, this section will show how to solve the minimization (3.7) and introduce the method to discretize the solution.

The solution of (3.7) is determined by alternatively minimizing the objective function: We alternate

- minimizing the objective function w.r.t. $\boldsymbol{p}$ with $\boldsymbol{u}, \boldsymbol{v}, \boldsymbol{\epsilon}, \boldsymbol{w}$ fixed,

- minimizing the objective function w.r.t. $\boldsymbol{w}$ with $\boldsymbol{u}, \boldsymbol{v}, \boldsymbol{\epsilon}, \boldsymbol{p}$ fixed,

- minimizing the objective function w.r.t. $\boldsymbol{v}$ with $\boldsymbol{u}, \boldsymbol{\epsilon}, \boldsymbol{p}, \boldsymbol{w}$ fixed,

- minimizing the objective function w.r.t. $\boldsymbol{\epsilon}$ with $\boldsymbol{u}, \boldsymbol{v}, \boldsymbol{p}, \boldsymbol{w}$ fixed,

- minimizing the objective function w.r.t. $\boldsymbol{u}$ with $\boldsymbol{v}, \boldsymbol{p}, \boldsymbol{\epsilon}, \boldsymbol{w}$ fixed.

Thus, we need to solve five subproblems denoted as " $\boldsymbol{w}$-subproblem", " $\boldsymbol{p}$-subproblem",

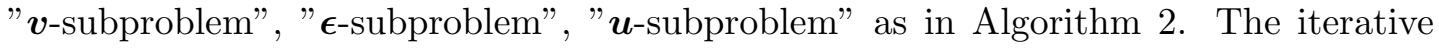
scheme is following:

"p-subproblem": Fix $\boldsymbol{u}, \boldsymbol{v}, \boldsymbol{\epsilon}, \boldsymbol{w}$ and

$$
\min _{\boldsymbol{p} \in Y}\left\{\|\boldsymbol{p}\|_{\ell_{1}}+\frac{\beta_{1}}{2}\left\|\boldsymbol{p}-\nabla_{\mathrm{d}} \boldsymbol{u}+\frac{\boldsymbol{\lambda}_{\mathbf{1}}}{\beta_{1}}\right\|_{\ell_{2}}^{2}\right\} .
$$

Let $\nabla_{\mathrm{d}}=\nabla^{+}$be the forward gradient operator. This (3.9) is solved by (cf. Proposition 3.6.1 in Appendix 3.6)

$$
\tilde{\boldsymbol{p}}_{1}=\operatorname{Shrink}\left(\partial_{1}^{+} \boldsymbol{u}-\frac{\boldsymbol{\lambda}_{\mathbf{1}, 1}}{\beta_{1}}, \frac{1}{\beta_{1}}\right) \quad \text { and } \quad \tilde{\boldsymbol{p}}_{2}=\operatorname{Shrink}\left(\partial_{2}^{+} \boldsymbol{u}-\frac{\boldsymbol{\lambda}_{\mathbf{1}, 2}}{\beta_{1}}, \frac{1}{\beta_{1}}\right),
$$

where $\partial_{m}^{+} \boldsymbol{u}=\left[\partial_{m}^{+} u[\boldsymbol{k}]\right]_{\boldsymbol{k} \in \Omega_{d}}, m \in\{1,2\}$.

"w-subproblem": Fix $\boldsymbol{u}, \boldsymbol{v}, \boldsymbol{\epsilon}, \boldsymbol{p}$ and

$$
\min _{\boldsymbol{w} \in \mathbb{R}^{\mathbb{I}} \mid}\left\{\mu_{1}\|\boldsymbol{w}\|_{\ell_{1}}+\frac{\beta_{2}}{2}\left\|\boldsymbol{w}-\mathcal{C}\{\boldsymbol{v}\}+\frac{\boldsymbol{\lambda}_{\mathbf{2}}}{\beta_{2}}\right\|_{\ell_{2}}^{2}\right\} .
$$

The solution of (3.11) at the scale $i$ and the orientation $l$ is (cf. Proposition 3.6.1 in Appendix 3.6)

$$
\tilde{\boldsymbol{w}}_{i, l}=\operatorname{Shrink}\left(\mathcal{C}\{\boldsymbol{v}\}-\frac{\boldsymbol{\lambda}_{\mathbf{2}, i, l}}{\beta_{2}}, \frac{\mu_{1}}{\beta_{2}}\right), \quad i, l \in \mathcal{I}
$$

"v-problem": Fix $\boldsymbol{u}, \boldsymbol{\epsilon}, \boldsymbol{p}, \boldsymbol{w}$ and

$$
\min _{\boldsymbol{v} \in X}\left\{\mu_{2}\|\boldsymbol{v}\|_{\ell_{1}}+\frac{\beta_{2}}{2}\left\|\boldsymbol{w}-\mathcal{C}\{\boldsymbol{v}\}+\frac{\boldsymbol{\lambda}_{\mathbf{2}}}{\beta_{2}}\right\|_{\ell_{2}}^{2}+\frac{\beta_{3}}{2}\left\|\boldsymbol{f}-\boldsymbol{u}-\boldsymbol{v}-\boldsymbol{\epsilon}+\frac{\boldsymbol{\lambda}_{\mathbf{3}}}{\beta_{3}}\right\|_{\ell_{2}}^{2}\right\} .
$$


This (3.13) is solved by (cf. Proposition 3.6.3 in Appendix 3.6)

$$
\tilde{\boldsymbol{v}}=\operatorname{Shrink}\left(\boldsymbol{A}, \frac{\mu_{2}}{\beta_{2}+\beta_{3}}\right)
$$

where

$$
\boldsymbol{A}=\frac{\mathcal{C}^{*}\left\{\beta_{2} \boldsymbol{w}+\boldsymbol{\lambda}_{\mathbf{2}}\right\}+\beta_{3}\left(\boldsymbol{f}-\boldsymbol{u}-\boldsymbol{\epsilon}+\frac{\boldsymbol{\lambda}_{\mathbf{3}}}{\beta_{3}}\right)}{\beta_{2}+\beta_{3}}
$$

"є-problem": Fix $\boldsymbol{u}, \boldsymbol{v}, \boldsymbol{p}, \boldsymbol{w}$ and

$$
\min _{\boldsymbol{\epsilon} \in X}\left\{G^{*}\left(\frac{\boldsymbol{\epsilon}}{\delta}\right)+\frac{\beta_{3}}{2}\left\|\boldsymbol{\epsilon}-\left(\boldsymbol{f}-\boldsymbol{u}-\boldsymbol{v}+\frac{\boldsymbol{\lambda}_{\mathbf{3}}}{\beta_{3}}\right)\right\|_{\ell_{2}}^{2}\right\} .
$$

This (3.16) is solved by (cf. Proposition 3.6.4 in Appendix 3.6)

$$
\tilde{\boldsymbol{\epsilon}}=\left(\boldsymbol{f}-\boldsymbol{u}-\boldsymbol{v}+\frac{\boldsymbol{\lambda}_{\mathbf{3}}}{\beta_{3}}\right)-\operatorname{CST}\left(\boldsymbol{f}-\boldsymbol{u}-\boldsymbol{v}+\frac{\boldsymbol{\lambda}_{\mathbf{3}}}{\beta_{3}}, \delta\right) .
$$

"u-problem": Fix $\boldsymbol{v}, \boldsymbol{p}, \boldsymbol{\epsilon}, \boldsymbol{w}$ and

$$
\min _{\boldsymbol{u} \in X}\left\{\frac{\beta_{1}}{2}\left\|\boldsymbol{p}-\nabla_{\mathrm{d}} \boldsymbol{u}+\frac{\boldsymbol{\lambda}_{\mathbf{1}}}{\beta_{1}}\right\|_{\ell_{2}}^{2}+\frac{\beta_{3}}{2}\left\|\boldsymbol{f}-\boldsymbol{u}-\boldsymbol{v}-\boldsymbol{\epsilon}+\frac{\boldsymbol{\lambda}_{\mathbf{3}}}{\beta_{3}}\right\|_{\ell_{2}}^{2}\right\} .
$$

This (3.18) is solved by (cf. Proposition 3.6.5 in Appendix 3.6)

$$
\tilde{u}[\boldsymbol{k}]=\operatorname{Re}\left[\mathcal{F}^{-1}\left\{\frac{D\left(e^{j \omega}\right)}{\beta_{3}+4 \beta_{1}\left[\sin ^{2}\left(\frac{\omega_{1}}{2}\right)+\sin ^{2}\left(\frac{\omega_{2}}{2}\right)\right]}\right\}\right][\boldsymbol{k}], \quad \forall \boldsymbol{k} \in \Omega_{d} \text { and } \boldsymbol{\omega} \in \Omega_{\omega} .
$$

and the vector form of $\tilde{u}[\boldsymbol{k}]$ is $\tilde{\boldsymbol{u}}=[\tilde{u}[\boldsymbol{k}]]_{\boldsymbol{k} \in \Omega_{d}}$.

The updated Lagrange multiplier $\boldsymbol{\lambda}_{\mathbf{1}}^{(n)}, \boldsymbol{\lambda}_{\mathbf{2}}^{(n)}$ and $\boldsymbol{\lambda}_{\mathbf{3}}^{(n)}$ in (3.8) are

$$
\left\{\begin{array}{l}
\boldsymbol{\lambda}_{\mathbf{1}, 1}^{(n)}=\boldsymbol{\lambda}_{\mathbf{1}, 1}^{(n-1)}+\gamma \beta_{1}\left(\tilde{\boldsymbol{p}}_{1}^{(n)}-\partial_{1}^{+} \tilde{\boldsymbol{u}}^{(n)}\right), \\
\boldsymbol{\lambda}_{\mathbf{1}, 2}^{(n)}=\boldsymbol{\lambda}_{\mathbf{1}, 2}^{(n-1)}+\gamma \beta_{1}\left(\tilde{\boldsymbol{p}}_{2}^{(n)}-\partial_{2}^{+} \tilde{\boldsymbol{u}}^{(n)}\right), \\
\boldsymbol{\lambda}_{\mathbf{2}, i, l}^{(n)}=\boldsymbol{\lambda}_{\mathbf{2}, i, l}^{(n-1)}+\gamma \beta_{2}\left(\tilde{\boldsymbol{w}}_{i, l}^{(n)}-\mathcal{C}_{i, l}\left\{\tilde{\boldsymbol{v}}^{(n)}\right\}\right), \\
\boldsymbol{\lambda}_{\mathbf{3}}^{(n)}=\boldsymbol{\lambda}_{\mathbf{3}}^{(n-1)}+\gamma \beta_{3}\left(\boldsymbol{f}-\tilde{\boldsymbol{u}}^{(n)}-\tilde{\boldsymbol{v}}^{(n)}-\tilde{\boldsymbol{\epsilon}}^{(n)}\right) .
\end{array}\right.
$$


The summary of the numerical implementation is presented in Algorithm 3.

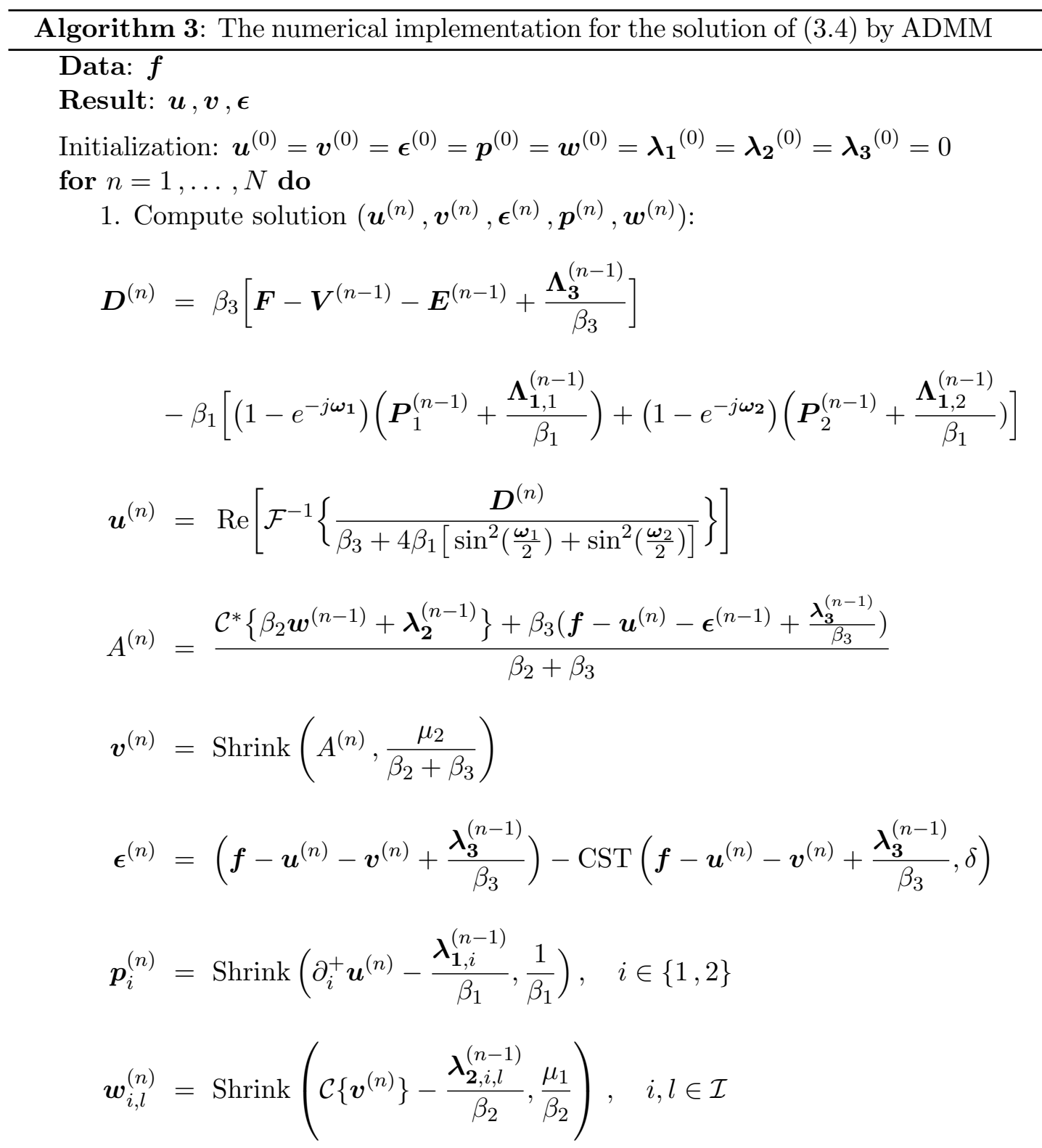

2. Update Lagrange multipliers $\left(\boldsymbol{\lambda}_{\mathbf{1}}{ }^{(n)}, \boldsymbol{\lambda}_{\mathbf{2}}{ }^{(n)}, \boldsymbol{\lambda}_{\mathbf{3}}{ }^{(n)}\right)$ :

$$
\begin{aligned}
& \boldsymbol{\lambda}_{\mathbf{1}, i}^{(n)}=\boldsymbol{\lambda}_{\mathbf{1}, i}^{(n-1)}+\gamma \beta_{1}\left(\boldsymbol{p}_{i}^{(n)}-\partial_{i}^{+} \boldsymbol{u}^{(n)}\right), \quad i \in\{1,2\} \\
& \boldsymbol{\lambda}_{\mathbf{2}, i, l}^{(n)}=\boldsymbol{\lambda}_{\mathbf{2}, i, l}^{(n-1)}+\gamma \beta_{2}\left(\boldsymbol{w}_{i, l}^{(n)}-\mathcal{C}_{i, l}\left\{\boldsymbol{v}^{(n)}\right\}\right), \quad i, l \in \mathcal{I} \\
& \boldsymbol{\lambda}_{\mathbf{3}}^{(n)}=\boldsymbol{\lambda}_{\mathbf{3}}^{(n-1)}+\gamma \beta_{3}\left(\boldsymbol{f}-\boldsymbol{u}^{(n)}-\boldsymbol{v}^{(n)}-\boldsymbol{\epsilon}^{(n)}\right)
\end{aligned}
$$

end

Note. $\left(\boldsymbol{F}, \boldsymbol{V}, \boldsymbol{E}, \boldsymbol{P}, \boldsymbol{\Lambda}_{\mathbf{1}}, \boldsymbol{\Lambda}_{\mathbf{3}}\right)$ are the discrete Fourier transform of $\left(\boldsymbol{f}, \boldsymbol{v}, \boldsymbol{\epsilon}, \boldsymbol{p}, \boldsymbol{\lambda}_{\mathbf{1}}, \boldsymbol{\lambda}_{\mathbf{3}}\right)$, respectively; and $\left(\boldsymbol{\omega}_{1}, \boldsymbol{\omega}_{\mathbf{2}}\right) \in[-\pi, \pi]$. 
For a given $\gamma$ in Algorithm 1, the solution of (3.3) is obtained by applying alternatively the above formulas in the subproblems. This is a convex program with the alternating minimization procedure. However, the choice of parameters $\left(\mu_{1}, \mu_{2}, \delta\right)$ and $\left(\beta_{1}, \beta_{2}, \beta_{3}\right)$ affects the solution. Since the texture information is the essential feature for the segmentation process, the parameters $\mu_{1}$ and $\mu_{2}$ are important, especially $\mu_{2}$, since it controls the sparsity of the fingerprint texture. In this context, $\mu_{2}$ is adaptively designed to cancel $\left(\beta_{2}+\beta_{3}\right)$ in the shrinkage operator (3.14) and it depends only on the maximum of $A$ and the constant $C$ as follows

$$
\mu_{2}=C\left(\beta_{2}+\beta_{3}\right) \cdot \max _{\boldsymbol{k} \in \Omega_{d}}(A[\boldsymbol{k}]),
$$

where $A[\boldsymbol{k}]$ is defined in (3.15). Since the fingerprint images are captured by four kinds of sensors, their qualities are different. Therefore, $C$ is obtained empirically from training sets for different types of sensors.

The parameter $\delta$ is used to remove the small scale objects (the noise). In order to reduce these noise in $v$ so that $v$ contains mainly the fingerprint pattern (cf. Figure 3.1 ), the noise is modeled as Gaussian and the parameter $\delta$ is determined from the asymptotic distribution of the maximum of the curvelet coefficients. Let $\left\|\mathcal{C}\left\{\boldsymbol{\epsilon}_{n}\right\}\right\|_{\infty}$ be the maximum of an i.i.d. sequence of Gaussian random variables $\boldsymbol{\epsilon}_{n} \in \Omega_{d} \sim \mathcal{N}\left(0, \sigma^{2}\right)$, where $n=N_{1} \times N_{2}$ is the level of discretization and $\mathcal{I}_{n}=\mathcal{I}$ are the frame elements. Based on the extreme value theory the limiting distribution of $\left\|\mathcal{C}\left\{\boldsymbol{\epsilon}_{n}\right\}\right\|_{\infty}$ is given by:

$$
\lim _{n \rightarrow \infty} \mathbf{P}\{\left\|\mathcal{C}\left\{\boldsymbol{\epsilon}_{n}\right\}\right\|_{\infty} \leq \underbrace{a_{n} z+b_{n}}_{:=\delta}\}=\exp \left(-e^{-z}\right), \quad z \in \mathbb{R},
$$

with $a_{n}:=\sigma \frac{1}{\sqrt{2 \log \left|\mathcal{I}_{n}\right|}}$ and $b_{n}:=\sigma \sqrt{2 \log \left|\mathcal{I}_{n}\right|}-\sigma \frac{\log \log \left|\mathcal{I}_{n}\right|+\log \pi}{2 \sqrt{2 \log \left|\mathcal{I}_{n}\right|}}$.

We rewrite (3.20) as

$$
\lim _{n \rightarrow \infty} \mathbf{P}\left\{\left\|\mathcal{C}\left\{\boldsymbol{\epsilon}_{n}\right\}\right\|_{\infty} \leq z\right\}=\exp \left(-e^{-\frac{z-b_{n}}{a_{n}}}\right), \quad z \in \mathbb{R} .
$$

Note that r.h.s in (3.21) depends on $n$, l.h.s does not. According to the extreme value behavior of the curvelet coefficients (cf. [31]), the threshold $\delta$ is defined as the $70 \%$ quantile of the limiting distribution, that is:

$$
\delta=\sigma \sqrt{2 \log |\mathcal{I}|}+\sigma \frac{2 z-\log \log |\mathcal{I}|-\log \pi}{2 \sqrt{2 \log |\mathcal{I}|}} \text { and } \quad z=-\log \log \left(\frac{1}{1-\alpha}\right),
$$

where $|\mathcal{I}|$ is total number of curvelet coefficients and $\sigma$ is commonly calculated from the first level of the Cohen-Daubechies-Feauveau $9 / 7$ wavelet high-frequency diagonal coefficient (HH1) (cf. [104]):

$$
\sigma=\frac{\operatorname{median}(|H H 1|)}{0.6745}
$$

Note that this approximation depends on the normality assumption of the noise, which may not always be true in practice. More sophisticated noise modeling is left for future investigation, in principle the threshold can always be obtained via simulation. 
The limiting distribution of the maximum of an i.i.d. sequence of Gaussian random variables is the Gumbel distribution, as in (3.20). Since the curvelet frame is overcomplete, the $\left\|\mathcal{C}\left\{\boldsymbol{\epsilon}_{n}\right\}\right\|_{\infty}$ is the maximum of dependent Gaussian random variables (curvelet coefficients). If the dependence is weak enough (see the conditions $D(n u)$ and $D^{\prime}(n u)$ ) in [105]), the limiting distribution is the same as in the i.i.d. situation. When considering the Cohen-Daubechies-Feauveau 9/7 wavelet coefficients instead, we are in the i.i.d. case and the asymptotics are standard (see Figure 3.3, second row). The influence of the dependence and the non-normalized curvelet coefficients is most likely the cause of the very slow convergence to the limit distribution, which causes the large distance between theoretical and the empirical distribution of the maximum (see Figure 3.3, third row).
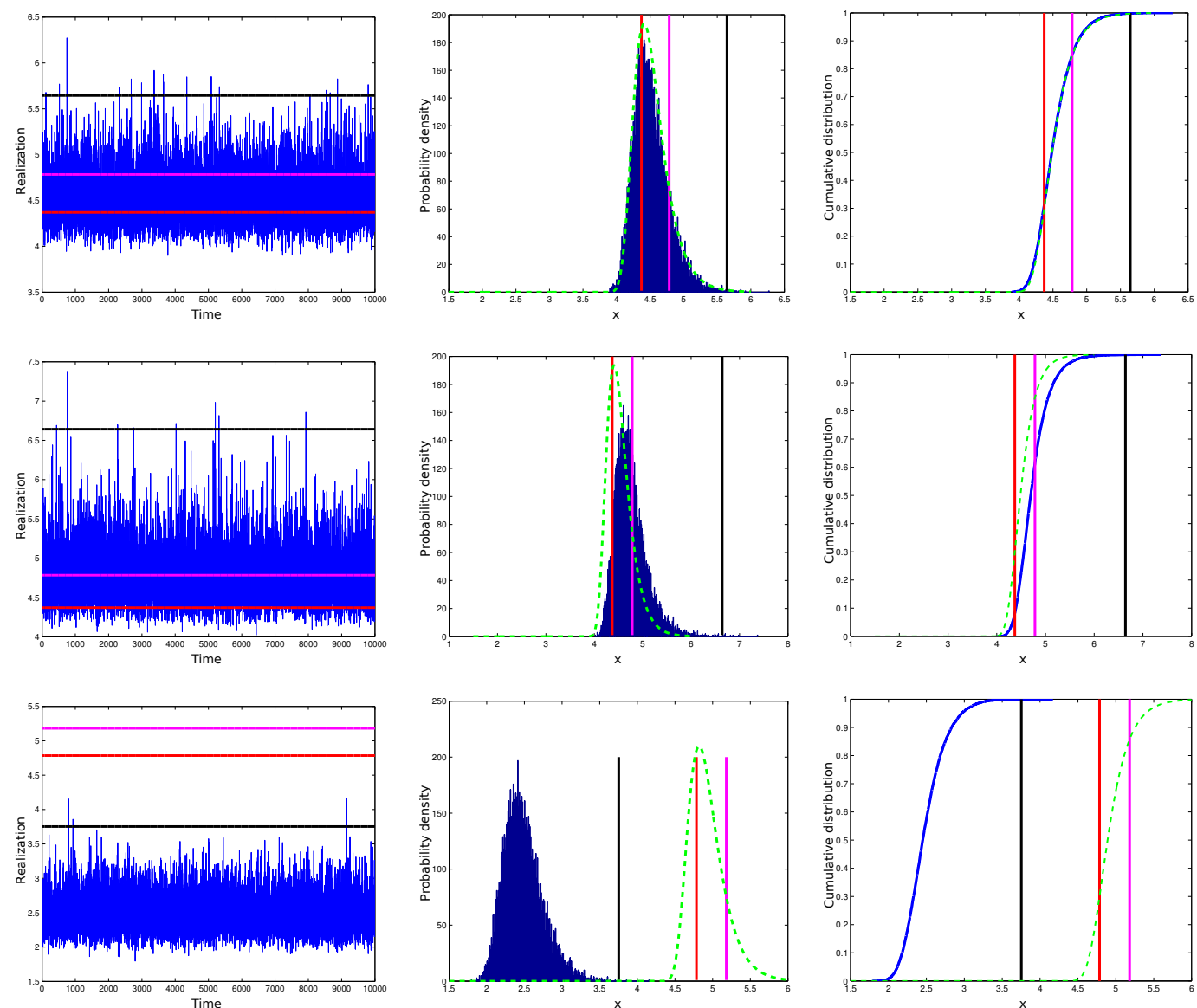

Figure 3.3: The plots f.l.t.r. in the first row are 10000 realizations of random variable $\left\|\boldsymbol{\epsilon}_{n}\right\|_{\infty}$, its histogram (blue) and its empirical cumulative distribution function (ecdf) (blue), respectively. Similarly, the second and third rows are 10000 realizations, histogram and ecdf of random variable $\left\|\mathcal{W}\left\{\boldsymbol{\epsilon}_{n}\right\}\right\|_{\infty}$ and $\left\|\mathcal{C}\left\{\boldsymbol{\epsilon}_{n}\right\}\right\|_{\infty}$, respectively. The magenta line is the universal threshold $\delta_{\text {ut }}=\sigma \sqrt{2 \log \left|\mathcal{I}_{n}\right|}$ (cf. [50]). The black line is the threshold with $90 \%$ quantile. The red line is the extreme value threshold $\delta$ in (3.22). The green dash curve is the Gumbel distribution. 


\subsubsection{Smoothness and Sparsity of the Extracted Texture}

The oscillation signal corresponding to fingerprint patterns is considered as a sparse and smooth texture which is decomposed by the G3PD model of the original fingerprint image $\boldsymbol{f}$ into three parts satisfying the constraint $\boldsymbol{f}=\boldsymbol{u}+\boldsymbol{v}+\boldsymbol{\epsilon}$ (cf. Figure 3.6), including: the piecewise-constant image $\boldsymbol{u}$ with TV-norm, the texture $\boldsymbol{v}$ with $\left\{G_{1,1}^{3 / 4}-\ell_{1}\right\}$ norms, and noise $\boldsymbol{\epsilon}$ with $G_{\infty, \infty}^{-3 / 4}$ norm.

In this section, we will analyze how $G_{1,1}^{3 / 4}$ and $\ell_{1}$ norms in (3.3) affect the smoothness and sparsity of the extracted texture $\boldsymbol{v}$. In order to do that, a closed form of $\boldsymbol{v}$ is found by putting (3.12) and (3.15) into (3.14), letting $\theta=\frac{\beta_{2}}{\beta_{2}+\beta_{3}}$ and the thresholds $T_{1}=\frac{\mu_{1}}{\beta_{2}}$ and $T_{2}=\frac{\mu_{2}}{\beta_{2}+\beta_{3}}$ :

$\tilde{\boldsymbol{v}}=\operatorname{Shrink}(\theta \underbrace{\left.\operatorname{Shrink}\left(\mathcal{C}\{\boldsymbol{v}\}-\frac{\boldsymbol{\lambda}_{\mathbf{2}}}{\beta_{2}}, T_{1}\right)+\frac{\boldsymbol{\lambda}_{\mathbf{2}}}{\beta_{2}}\right\}}_{:=\boldsymbol{v}_{\text {smooth }} \approx \operatorname{CST}\left(\boldsymbol{v}, T_{1}\right)}+(1-\theta) \underbrace{\left(\boldsymbol{f}-\boldsymbol{u}-\boldsymbol{\epsilon}+\frac{\boldsymbol{\lambda}_{\mathbf{3}}}{\beta_{3}}\right)}_{:=\boldsymbol{v}_{\text {update }}}, T_{2})$,

where $\operatorname{CST}(\cdot, \cdot)$ is the curvelet shrinkage operator (see Appendix 3.6). We see that the estimated texture $\tilde{\boldsymbol{v}}$ contains two shrinkage operators: respectively, the inside and the outside correspond to the smoothness and sparseness terms resulting from $\|\mathcal{C} \boldsymbol{v}\|_{\ell_{1}}$ and $\|\boldsymbol{v}\|_{\ell_{1}}$ in (3.3). (cf. Figure 3.4 for the effects of the smoothness and sparseness of $\boldsymbol{v}$ through different iterations). Note that the estimates $(\boldsymbol{u}, \boldsymbol{v}, \boldsymbol{n})$ (cf. Figure 3.4: 1st row, 2 nd to 4 th columns) is obtained at 20 iterations (approximately the starting convergence point, cf. 1st column of 2nd row in Figure 3.5). These effects can be obviously seen in the binarized texture (Figure 3.4, first row third column). The parameter $\theta \in(0,1)$ in (3.23) plays as a regularized parameter to make a balance between the smoothing term $\boldsymbol{v}_{\text {smooth }}$ and the updated term $\boldsymbol{v}_{\text {update }}$.

Figure 3.5 shows the effect of the curvelet smoothing measurement in $G_{1,1}^{3 / 4}$ for texture $\boldsymbol{v}$, i.e. $\|\mathcal{C}\{\boldsymbol{v}\}\|_{\ell_{1}}$ in (3.1), and its comparison with wavelet smoothing term in $B_{1,1}^{1}$, i.e. $\|\mathcal{W}\{\boldsymbol{v}\}\|_{\ell_{1}}$, and without smoothing measurement. The estimate texture $\boldsymbol{v}$ with $\|\mathcal{W}\{\boldsymbol{v}\}\|_{\ell_{1}}$ in (3.1) is worse (in the sense of smoothness and sparseness) than in $G_{1,1}^{3 / 4}$, i.e. $\|\mathcal{C}\{\boldsymbol{v}\}\|_{\ell_{1}}$ (cf. 1st and 2nd columns of 3rd and 4th rows in Figure 3.5). Luckily, in this example, the performance of segmentation is better due to the effect of the morphological operator, but the roburstness doesnot satisfy. Moreover, it contradicts our assumption for texture $\boldsymbol{v}$, i.e. $\boldsymbol{v}$ should be as smooth and sparse as possible. In order to evaluate the convergence of the algorithm, we denote the relative error of texture $\boldsymbol{v}$ through different iterations $n$ as

$$
\operatorname{Err}_{\boldsymbol{v}}^{(n)}=\frac{\left\|\boldsymbol{v}^{(n)}-\boldsymbol{v}^{(n-1)}\right\|_{\ell_{2}}}{\left\|\boldsymbol{v}^{(n-1)}\right\|_{\ell_{2}}}
$$

In Figure 3.5, we see that if there is no smoothing measurement, the convergence is much slower than the others (cf. 2nd row) and the algorithm tends to eliminate texture (cf. 3rd and 4th columns of 3rd and 4th rows). In particular, at 50 iteration, $\boldsymbol{v}$ still does not converge and the segmentation error is higher than without smoothing term 
at 20 iterations (cf. 3rd and 4th columns of 1st row). Note that for the smoothing measurement $\|\mathcal{C}\{\boldsymbol{v}\}\|_{\ell_{1}}$, the proposed method starts to converge at around 20 iteration. Hence, the estimate of $\boldsymbol{v}$ (or its binarization and segmented image) with between 20 and 50 iterations are almost similar (cf. Figure 3.4 (a, c, e) and 1st column of Figure $3.5)$.

\subsubsection{Morphological Operations}

The smooth and sparse texture $\boldsymbol{v}$ is obtained by the combination of $G_{1,1}^{3 / 4}$ and $\ell_{1}$ norms, simultaneously. Post-processing, which is similar to [1], is applied to obtain the ROI. However, the threshold is simply set to " 0 " for texture binarization instead because it uses the advantage of sparse solution $\boldsymbol{v}$ (cf. Figure 3.1). In other words, it is similar to project the thresholding value to the parameter $\mu_{2}$ that has been desired adaptively to intensity of image by (3.19).

\subsection{Evaluation: Benchmark, Protocol and Experimental Results}

\subsubsection{Benchmark and Evaluation Metric}

The publicly available fingerprint images of the FVC competitions from 2000, 2002 and 2004 are used as benchmark for evaluating segmentation performance. Each competition consists of four databases: three databases are acquired from real fingerprints and the fourth database of each competition is synthetically generated. In total, there are 12 databases and each database contains 880 images ( 80 for training and 800 for testing). The ground truth segmentation has been manually marked for these 10560 images as described in [1].

Let $N_{1}$ and $N_{2}$ be the width and height of image $\boldsymbol{f}$ in pixels. Let $M_{f}$ be number of pixels which are marked as foreground by human experts and estimated as background by an algorithm (missed/misclassified foreground). Let $M_{b}$ be number of pixels which are marked as background by human experts and estimated as foreground by an algorithm (missed/misclassified background). The average total error per image is defined as

$$
E r r=\frac{M_{f}+M_{b}}{N_{1} \times N_{2}} .
$$

\subsubsection{Parameter Selection}

Parameters for all methods considered in the comparison are selected on the training set of 80 images for each database. More specifically, those parameters are chosen to minimize the segmentation error defined in (3.25) for the respective training set. Choosing the parameters for each database is appropriate, because the nine databases consisting of real fingerprints have been acquired using nine different sensors and the images of each database have sensor-specific properties. The parameter selection for the FDB [1], GFB [8], HCR [9], MVC [7] and STFT [10] methods are discussed in [1]. 


\begin{tabular}{|c|l|}
\hline Parameters & Description \\
\hline$N$ & the number of iterations in the Algorithm 1. \\
\hline$\mu_{1}$ & $\begin{array}{l}\text { the regularized parameter for } \ell_{1} \text { norm of curvelet coefficients } \mathcal{C}\{\boldsymbol{v}\} \\
\text { in Eq. }(3.2) .\end{array}$ \\
\hline$C$ & $\begin{array}{l}\text { the adaptive constant in Eq. (3.19) for the regularized parameter } \mu_{2} \\
\text { in } \ell_{1} \text { norm of } \boldsymbol{v} \text { in Eq. (3.2). }\end{array}$ \\
\hline$\beta_{1}, \beta_{2}, \beta_{3}$ & the parameters in the augmented Lagrangian function (3.5). \\
\hline$\gamma$ & the speed rate of the updated Lagrange multipliers in Eq. (3.5). \\
\hline$s$ & the window size of the block in the postprocessing step in [1, Eq. (8)]. \\
\hline$t$ & a constant for selecting the morphology threshold $T$ in $[1$, Eq. (8)]. \\
\hline$b$ & the number of the neighboring blocks in [1, Eq. (8)]. \\
\hline$p$ & the mirror boundary condition to avoid the boundary effect. \\
\hline
\end{tabular}

Table 3.1: Overview over all parameters for the global three-part decomposition (G3PD) method for fingerprint segmentation. Values are reported in Table 3.2 .

\begin{tabular}{|c|c|c|c|}
\hline FVC & DB & $C$ & $\beta_{2}$ \\
\hline 2000 & 1 & 0.045 & 0.0005 \\
& 2 & 0.045 & 0.0100 \\
& 3 & 0.055 & 0.0010 \\
& 4 & 0.025 & 0.0010 \\
\hline 2002 & 1 & 0.020 & 0.0010 \\
& 2 & 0.035 & 0.0005 \\
& 3 & 0.070 & 0.0010 \\
& 4 & 0.020 & 0.0500 \\
\hline 2004 & 1 & 0.015 & 0.1000 \\
& 2 & 0.025 & 0.0010 \\
& 3 & 0.035 & 0.0010 \\
& 4 & 0.035 & 0.0005 \\
\hline
\end{tabular}

Table 3.2: Overview over the parameters learned on the training set. The other eight parameters are $\mu_{1}=1, \beta_{1}=\beta_{3}=\gamma=10^{-3}, s=9, t=5, b=6$ and $p=15$ for all databases. 
For the proposed G3PD method, the involved parameters are summarized in Table 3.1 and the values of the learned parameters are reported in Table 3.2. In a reasonable amount of time, a number of conceivable parameter combinations was tried on the training set.

For different numbers of iterations, we have applied the following training scheme:

- Firstly, $C$, an adaptive constant for $\mu_{2}$ in (3.19) to define a threshold for the sparseness of $\boldsymbol{v}$, is trained while fixing the other parameters.

- Secondly, with the obtained $C$, we train the other parameters one by one while fixing the rest.

In our experiments, the minimum error on the training set averaged over all 12 databases is obtained for $N=4$ iterations (cf. Figure 3.13b). Given $N=4$ iterations, the training error is further reduced by choosing $\beta_{2}$ (cf. Figure 3.13a). Figure 3.10 displays the fingerprint segmentation result for different numbers of iterations. In these practical applications of our proposed model, stopping before convergence leads to better segmentation results which are also influenced by the combination with the morphological operations.

Note that the solution of $(\boldsymbol{u}, \boldsymbol{v}, \boldsymbol{\epsilon})$ depends severely on the choices of $\left(\mu_{1}, \mu_{2}, \delta\right)$, as well as the parameters of the optimization step $\left(\beta_{1}, \beta_{2}, \beta_{3}, \gamma\right)$. To achieve a good decomposition in which cartoon, texture and noise are separated is difficult in practice, because there are no models for both the noise and the texture. Fortunately, this chapter focuses on the segmentation of fingerprint images for which the texture $\boldsymbol{v}$ is important. After the decomposition, there can still be pattern contents in the cartoon image $\boldsymbol{u}$ and the noise image $\boldsymbol{\epsilon}$ (see Figure 3.1), but the important aspect is that the texture image $\boldsymbol{v}$ is adequate for segmentation.

The choice of aforementioned parameters balances the amount of pattern in the texture image with the smoothness of the cartoon image. Selecting parameters which increase the smoothness of the cartoon image $\boldsymbol{u}$, also tend to cause the halo effect in the texture image $\boldsymbol{v}$. We observe that especially $\beta_{1}$ influences this trade-off: if $\boldsymbol{u}$ contains only homogeneous regions (cf. Figure 3.6d), it tends to generate the halo effect on the boundary of fingerprint pattern in $\boldsymbol{v}$ (cf. Figure 3.6e). Particularly, the halo effect results from the blurred homogeneous region $\boldsymbol{u}$. However, this effect impacts the segmentation error which is obvious as depicted in Figure 3.11 for different iteration with the same $\beta_{1}=0.06$. In order to reduce this effect in $\boldsymbol{v}$, the parameters are chosen such that the algorithm assign "enough" texture to $\boldsymbol{v}$. Hence, $\boldsymbol{u}$ and $\boldsymbol{\epsilon}$ can contain some partial textures, but this yields better a segmentation performance as illustrated in Figure 3.10.

Let us consider the comparison of the proposed model with the standard ROF TV $-L_{2}$ model [72] and the TV $-L_{1}$ model [106] for feature decomposition (see Figure 3.9). For simplicity, let $\lambda_{\mathrm{TV} L_{2}}$ and $\lambda_{\mathrm{TV} L_{1}}$ be the regularization parameters for $\mathrm{TV}-L_{2}$ and TV $-L_{1}$, respectively. The ROF TV $-L_{2}$ model has been introduced by [72] for the purpose of image denoising. The ROF model has been designed to obtain a smooth cartoon image $\boldsymbol{u}$. For fingerprint image segmentation we are interested in a texture image which is as useful as possible in terms of a feature for segmentation. However, the ROF model or the TV $-L_{1}$ model cannot produce a sparse and smooth texture 
image from a noisy fingerprint image $f$ no matter how the corresponding parameter is selected. On the one hand, if the ROF model decomposes $f$ into a very smooth cartoon image $\boldsymbol{u}$, than $\boldsymbol{v}$ contains both noise and texture. On the other hand, for a different choice of $\lambda_{\mathrm{TV} L_{2}}$ or $\lambda_{\mathrm{TV} L_{1}}, \boldsymbol{v}$ contains mostly noise and $\boldsymbol{u}$ includes texture and large scale objects. In neither of the two situations, $\boldsymbol{u}$ or $\boldsymbol{v}$ is useful as a feature for fingerprint segmentation. A comparison of the G3PD method with TV $-L_{1}$ and $\mathrm{TV}-L_{2}$ two-part decomposition is shown in Figure 3.9. Zhang et al. [23] have tried to solve this problem by proposing a locally adaptive two-part decomposition which also takes the orientation of the pattern into account.

Aujol and Chambolle [101] introduced a model for three-part decomposition which can produce a texture image $\boldsymbol{v}$ using the G-norm. For fingerprint image segmentation we have adopted their approach into our TV $-G_{1,1}^{3 / 4}-\ell_{1}-G_{\infty, \infty}^{-3 / 4}$ model with the goal of obtaining a texture image $\boldsymbol{v}$ which is both smooth and sparse. The smoothness is measured by the $G_{1,1}^{3 / 4}$-norm (corresponding to the shrinkage operator of curvelet coefficients) and the sparsity is measured by the $\ell_{1}$-norm (corresponding to shrinkage operator in the spatial domain).

In summary, the proposed G3PD method yields a satisfactory performance judged by visual inspection (see Figure 3.8 for one example from each database) and it outperforms the other methods on ten of twelve databases. This demonstrates the robustness of the G3PD method for fingerprint segmentation. Figure 3.16 - 3.24 show the comparison of the proposed G3PD method with the other approaches.

\subsection{Conclusions}

We have presented a global framework for the fingerprint segmentation problem which is to separate the foreground from background based on texture analysis. We have proposed the G3PD method for three-part decomposition of fingerprint images. The texture pattern is analyzed under the variational approach considering sparsity and smoothness at the same time: with the $\ell_{1}$-norm for sparsity and curvelet type decomposition space $G_{1,1}^{3 / 4}$ for smoothness. The resulting texture image is binarized and postprocessed by morphology to obtain the region of interest.

We have proposed a model for three-part decomposition which also takes into account the nature of the texture occurring in real fingerprint images. Fingerprint images are characterized by a smooth, curved and oriented pattern which has a sparse representation in certain transform domains.

The G3PD method is somewhat similar in spirit to the FDB method [1] which also makes allowance for the properties of fingerprint patterns. Frequencies occurring in real fingerprints are mostly located in a specific range in the Fourier domain and the corresponding texture is extracted by an elaborate bandpass filtering process involving forward prediction, proximity operator and backward projection. Similarly, the three-part decomposition can be regarded as lowpass, bandpass and highpass filtering of signals corresponding to $\boldsymbol{u}, \boldsymbol{v}$ and $\boldsymbol{\epsilon}$, respectively (see images (g-i) in Figure 3.6). Figure 3.7 shows the evolution of $(\boldsymbol{u}, \boldsymbol{v}, \boldsymbol{\epsilon})$ and their spectrums at iterations $n=1,4,7,10$. This illustrates the connection between classical bandpass filtering in 


\begin{tabular}{|c|c|c|c|c|l|l|l|}
\hline FVC & DB & GFB $[8]$ & HCR [9] & MVC [7] & STFT [10] & FDB [1] & G3PD \\
\hline 2000 & 1 & 13.26 & 11.15 & 10.01 & 16.70 & $\mathbf{5 . 5 1}$ & 5.69 \\
& 2 & 10.27 & 6.25 & 12.31 & 8.88 & $\mathbf{3 . 5 5}$ & 4.10 \\
& 3 & 10.63 & 7.80 & 7.45 & 6.44 & 2.86 & $\mathbf{2 . 6 8}$ \\
& 4 & 5.17 & 3.23 & 9.74 & 7.19 & 2.31 & $\mathbf{2 . 0 6}$ \\
\hline 2002 & 1 & 5.07 & 3.71 & 4.59 & 5.49 & 2.39 & $\mathbf{1 . 7 2}$ \\
& 2 & 7.76 & 5.72 & 4.32 & 6.27 & 2.91 & $\mathbf{2 . 8 3}$ \\
& 3 & 9.60 & 4.71 & 5.29 & 5.13 & 3.35 & $\mathbf{3 . 2 7}$ \\
& 4 & 7.67 & 6.85 & 6.12 & 7.70 & 4.49 & $\mathbf{3 . 6 3}$ \\
\hline 2004 & 1 & 5.00 & 2.26 & 2.22 & 2.65 & 1.40 & $\mathbf{0 . 8 8}$ \\
& 2 & 11.18 & 7.54 & 8.06 & 9.89 & 4.90 & $\mathbf{4 . 6 2}$ \\
& 3 & 8.37 & 4.96 & 3.42 & 9.35 & 3.14 & $\mathbf{2 . 7 7}$ \\
& 4 & 5.96 & 5.15 & 4.58 & 5.18 & 2.79 & $\mathbf{2 . 5 3}$ \\
\hline Avg. & & 8.33 & 5.78 & 6.51 & 7.57 & 3.30 & $\mathbf{3 . 0 6}$ \\
\hline
\end{tabular}

Table 3.3: Error rates (average percentage of misclassified pixels averaged over 800 test images per database) computed using the manually marked ground truth segmentation and the estimated segmentation by these methods: a Gabor filter bank (GFB) response based method by Shen et al. [8], a Harris corner response (HCR) based approach by Wu et al. [9], a method by Bazen and Gerez using local gray-level mean, variance and gradient coherence (MVC) as features [7], a method applying short time Fourier transforms (STFT) by Chikkerur et al. [10], the factorized directional bandpass (FDB) [1] and the proposed method based on the TV $-G_{1,1}^{3 / 4}-\ell_{1}-G_{\infty, \infty}^{-3 / 4}$ model. 
the Fourier domain and the variational approach.

In conclusion, we have performed an extensive comparison of the G3PD method with five state-of-the-art fingerprint segmentation algorithms on a large benchmark with a variety of different challenges and have found that the G3PD method outperforms its competitors on ten out of twelve database in terms of segmentation accuracy.

Our future work includes to work on modifications of the TV $-G_{1,1}^{3 / 4}-\ell_{1}-G_{\infty, \infty}^{-3 / 4}$ model in order to adopt a multiscale (or locally adaptive) approach for image decomposition. This work opens the way for further research in areas like latent fingerprint segmentation in which we deal additionally with other kinds of noise like large scale structure noise, or to better deal with the few low-quality examples which still pose problems to the method (see Figure 3.15). We believe that further improvements can be achieved by combining the G3PD method with additional features, e.g. the texture image obtained by the FDB method. 


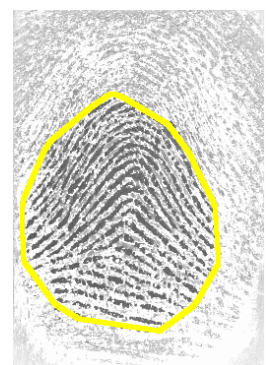

(a)

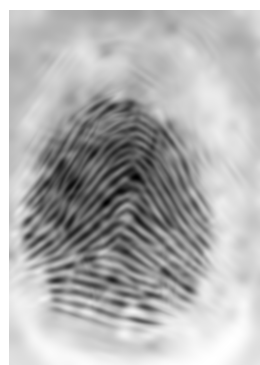

(b)

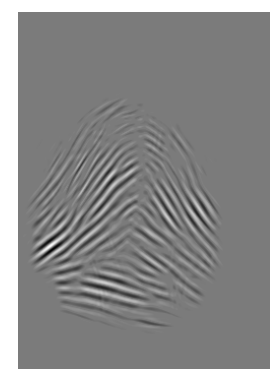

(c)

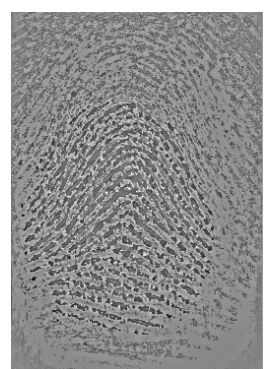

(d)

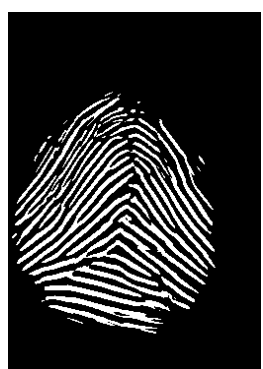

(e)

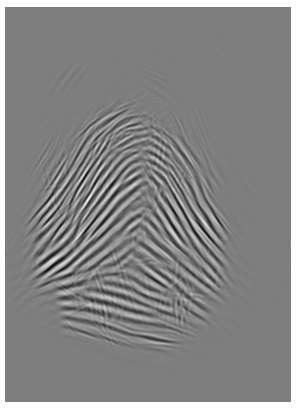

(f) $k=3$
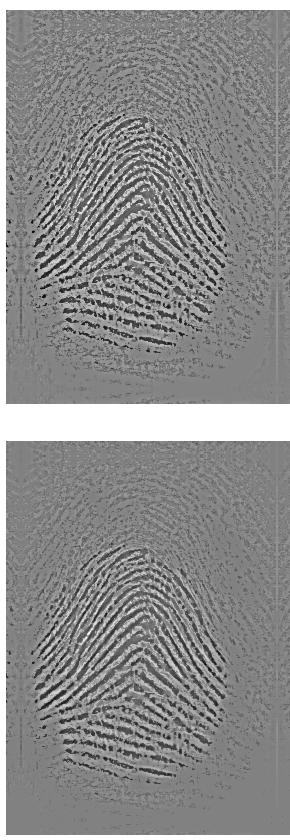

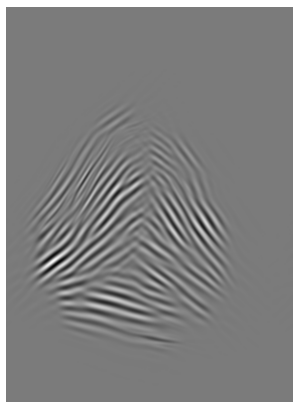

(g) $k=6$
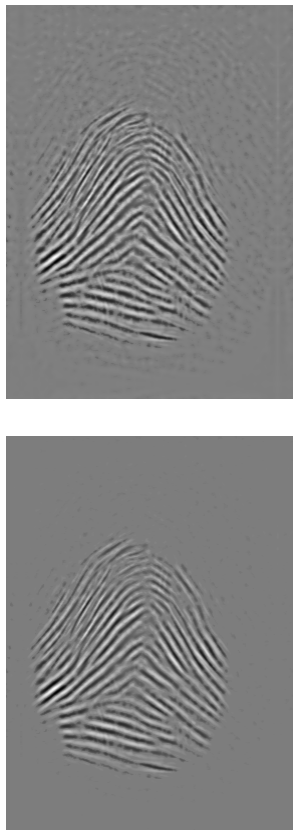

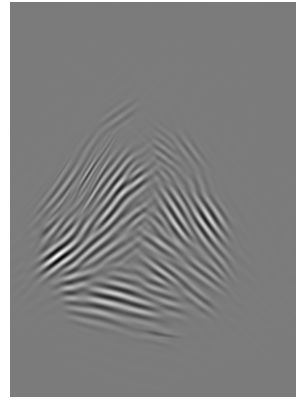

(h) $k=8$
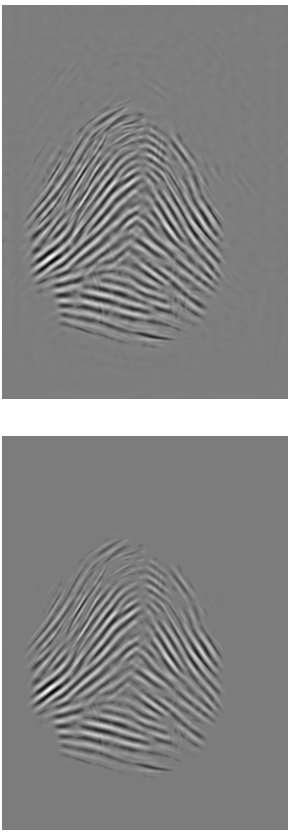

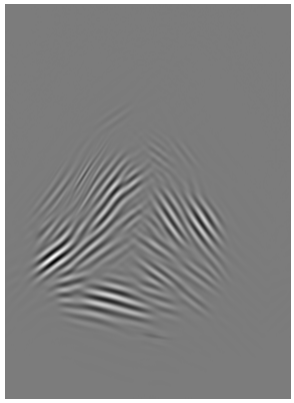

(i) $k=20$
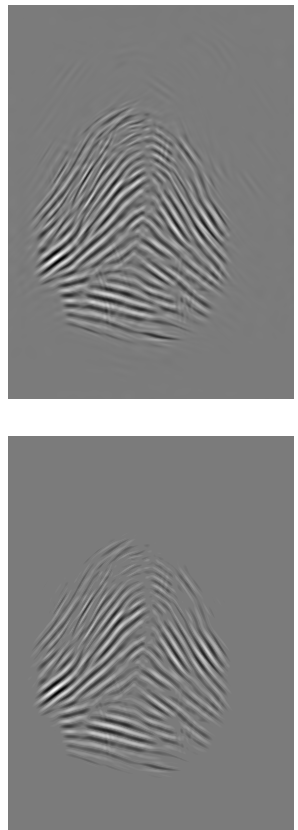

Figure 3.4: (a) the original image (the yellow line is the boundary of the ROI); (b, c, d) the estimates $(\boldsymbol{u}, \boldsymbol{v}, \boldsymbol{\epsilon})$ at 20 iterations, respectively; (e) the smooth and sparse binarized texture is obtained by setting the non-zeros coefficients of $\boldsymbol{v}$ (c) to 1 . The second row is $\boldsymbol{v}_{\text {smooth }}$ in (3.23), the smooth term of $\boldsymbol{v}$, in different iteration $k=$ $\{3,6,8,20\}$ which is obtained by the shrinkage operator of the curvelet coefficient (correspond to $\|\mathcal{C}\{\boldsymbol{v}\}\|_{\ell_{1}}$ in (3.1)). The third rows is $\boldsymbol{v}_{\text {update }}$ in (3.23). Both of them are balanced by the parameter $\theta$ which follows by the shrinkage operator to obtain the the sparse texture $\tilde{\boldsymbol{v}}$ in the fourth row (correspond to $\|\boldsymbol{v}\|_{\ell_{1}}$ in (3.1)). 


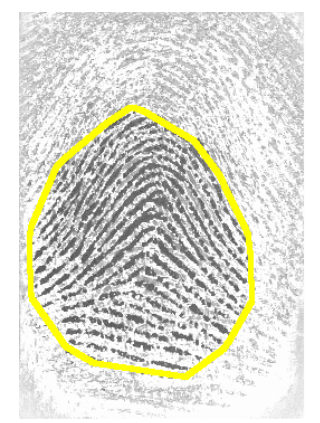

(a) $\operatorname{Err}=3.65$
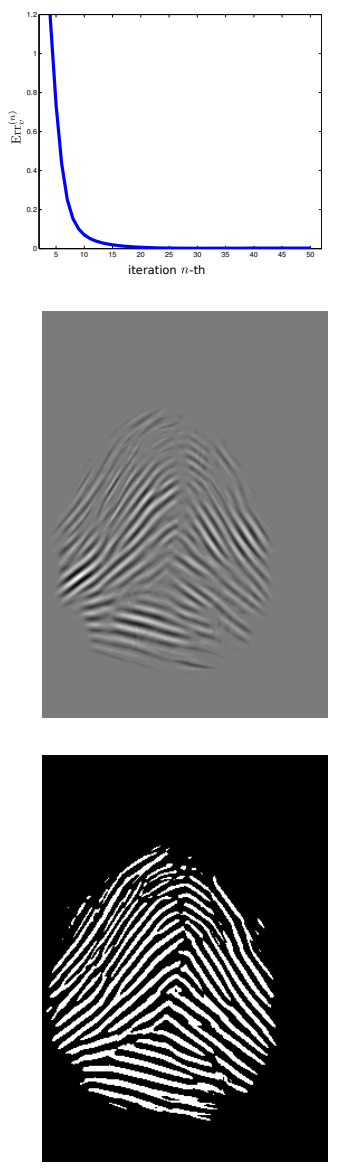

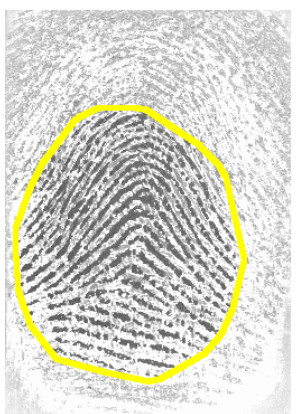

(b) $\operatorname{Err}=2.45$
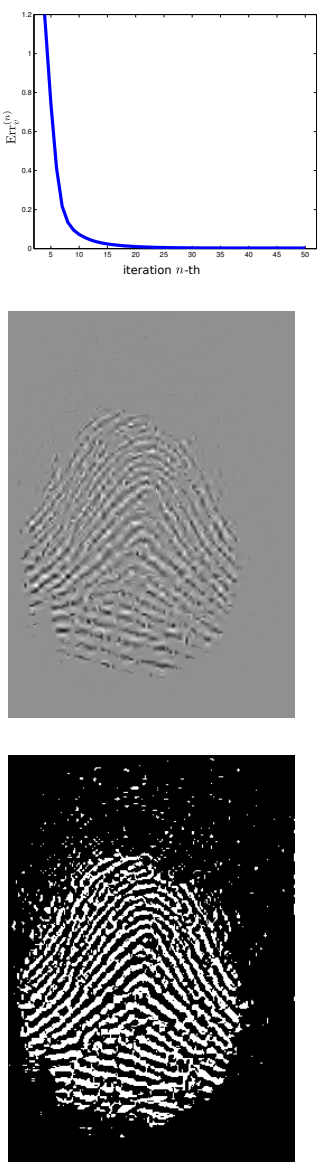

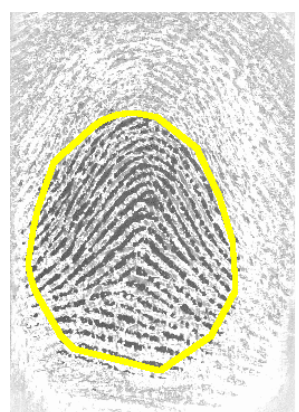

(c) $\operatorname{Err}=7.4$

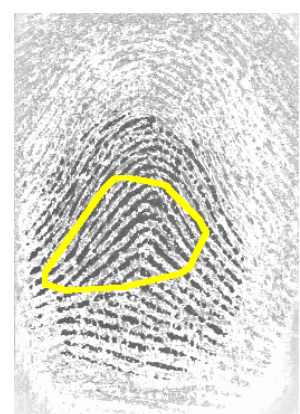

(d) $\operatorname{Err}=31.49$
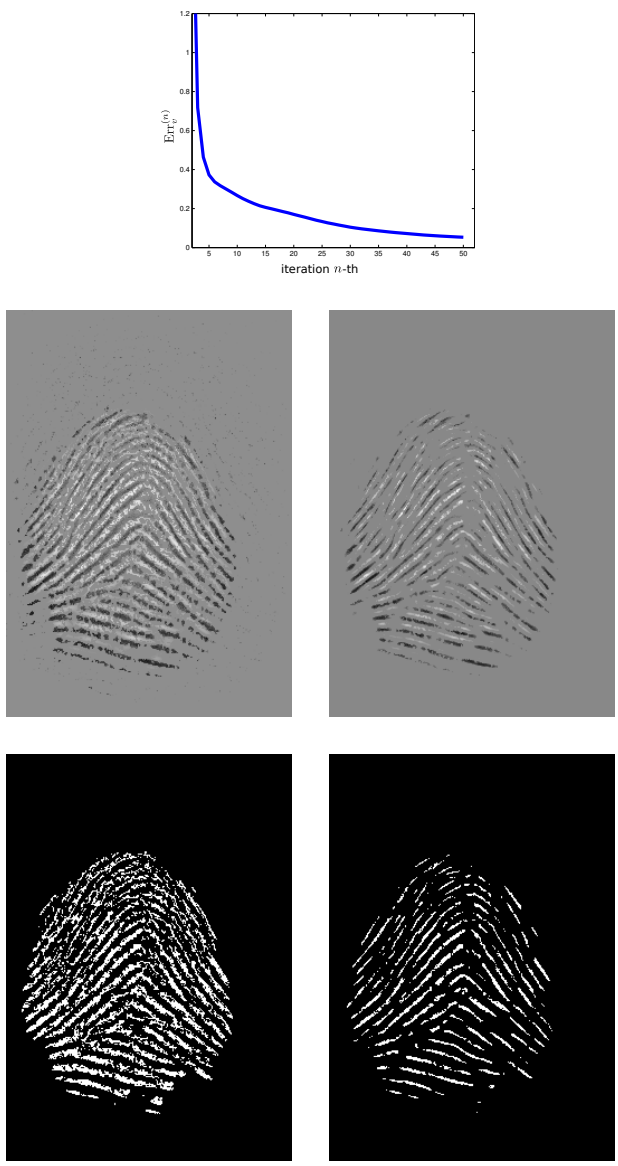

Figure 3.5: The effect of the smoothness term for $\boldsymbol{v}$ in (3.1). 1st column: $\ell_{1}$ norm of curvelet coefficients, i.e. $\|\mathcal{C}\{\boldsymbol{v}\}\|_{\ell_{1}}$, with 50 iterations. 2nd column: $\ell_{1}$ norm of wavelet coefficients, i.e. $\|\mathcal{W}\{\boldsymbol{v}\}\|_{\ell_{1}}$, with 50 iterations. 3rd and 4th columns: without smoothing term (no $\|\mathcal{C}\{\boldsymbol{v}\}\|_{\ell_{1}}$ in (3.1)) at 20 and 50 iterations, respectively. 1st row: the segmented images. 2nd row: the plots of convergence for $\boldsymbol{v}$ with curvelet (a), wavelet (b), without smoothing terms (c \& d). 3rd \& 4th rows: the estimated texture $\boldsymbol{v}$ and their binarization, respectively. It shows that the convergence without smoothing term is too slow and texture starts being destroyed in comparison with the others. 


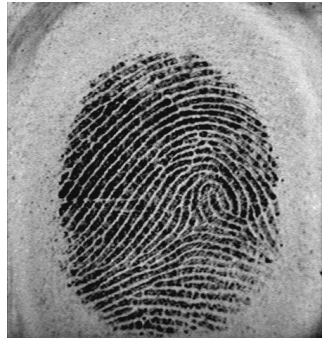

(a) $f$

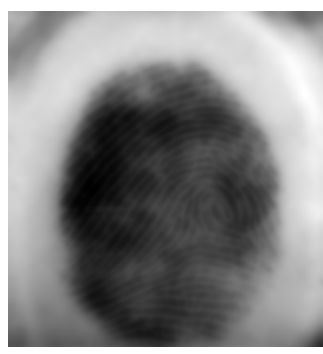

(d) $\boldsymbol{u}$

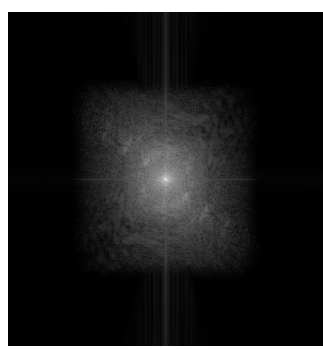

(g) $U\left(e^{j \boldsymbol{\omega}}\right)$

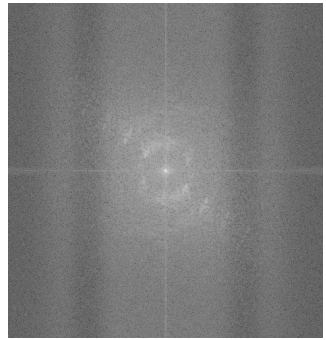

(b) $F\left(e^{j \omega}\right)$

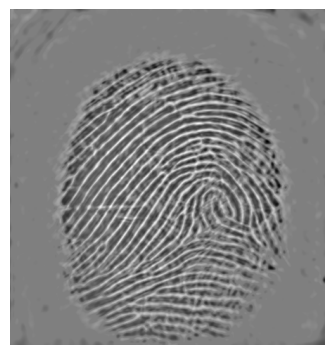

(e) $\boldsymbol{v}$

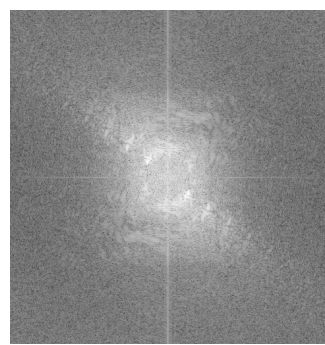

(h) $V\left(e^{j \boldsymbol{\omega}}\right)$

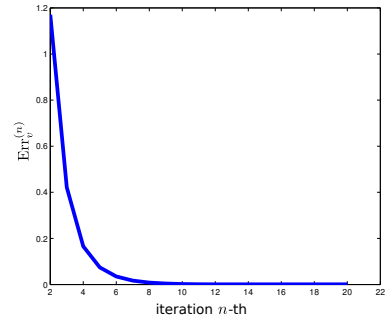

(c) $\operatorname{Err}_{v}$

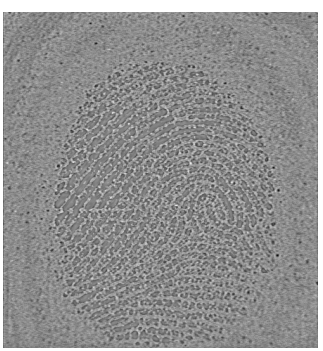

(f) $\epsilon$

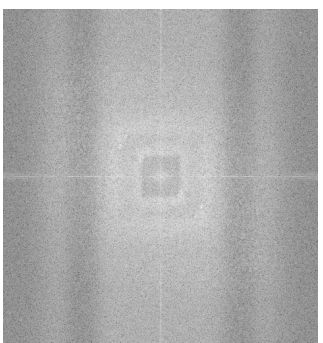

(i) $E\left(e^{j \boldsymbol{\omega}}\right)$

Figure 3.6: The decomposition model with $\mu_{1}=1$, iteration $=20$, level $=5, \beta_{1}=0.06, \beta_{2}=$ $\beta_{3}=\gamma=10^{-3}$. The original image and its Fourier spectrum are in (a) and (b), respectively. The relative error of $\boldsymbol{v}$ in (c), i.e. $\operatorname{Err}_{\boldsymbol{v}}^{(n)}$, shows that the algorithm starts to converge after 10 iterations. The second and third rows display the homogeneous, texture and noise, together with their Fourier spectrums, respectively. We see that the algorithm can separate $\boldsymbol{u}, \boldsymbol{v}$ and $\boldsymbol{\epsilon}$ with this choice of parameters, especially for the homogeneous region. In Fourier domain (cf. third row), $\boldsymbol{u}, \boldsymbol{v}$ and $\boldsymbol{\epsilon}$ are similar to the responses after lowpass, bandpass and highpass filters. Especially, Figure (e) and (f) shows that the fingerprint pattern mostly stays in a specific range of frequencies in bandpass filter which has been reported in [1]. 


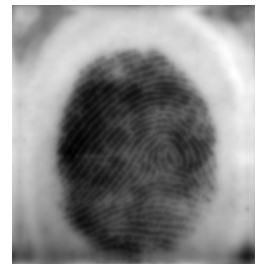

(a) $k=1$
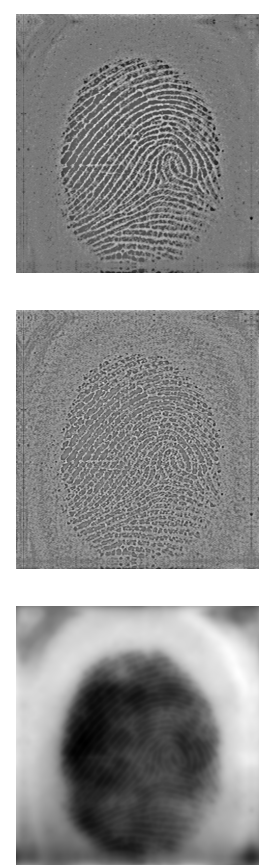

(m) $k=7$
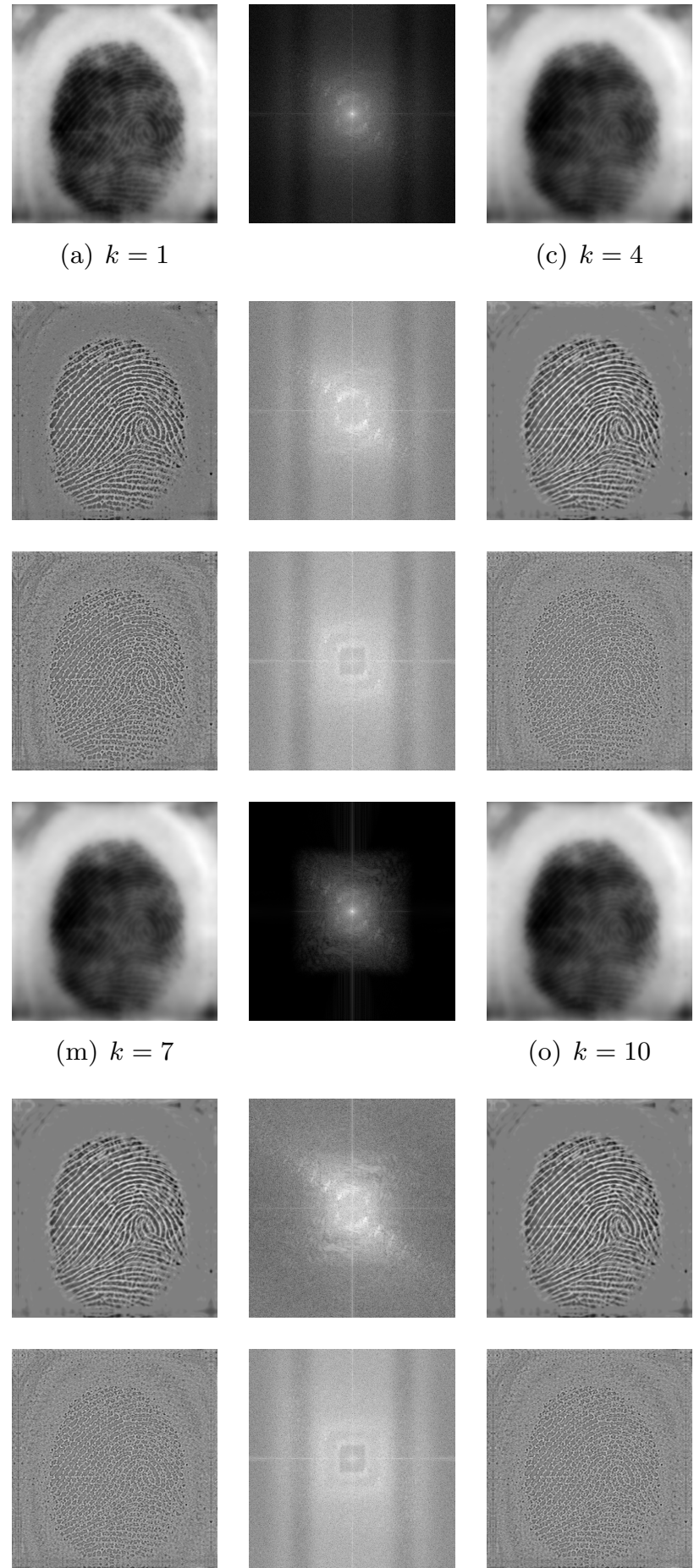

(c) $k=4$
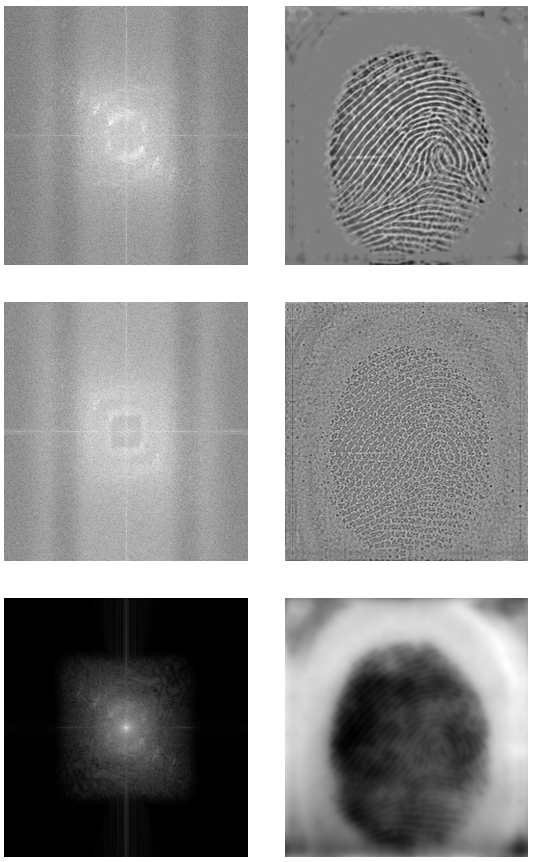

(o) $k=10$
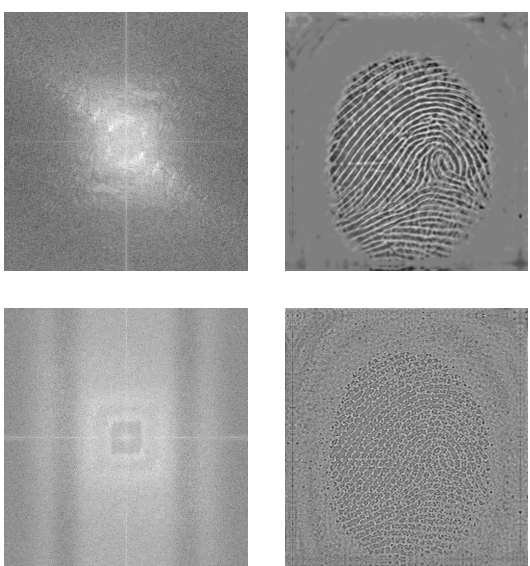
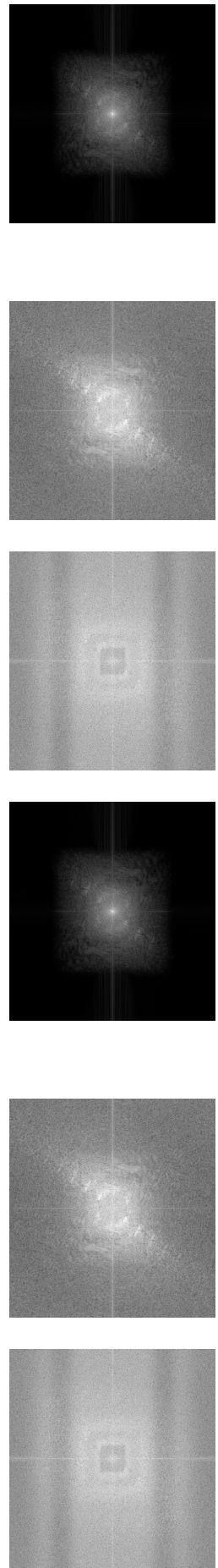

Figure 3.7: The evolution of $(\boldsymbol{u}, \boldsymbol{v}, \boldsymbol{\epsilon})$ with $n=1,4,7,10$ and their Fourier coefficients at 10-th iteration with $\beta_{1}=0.06$. 

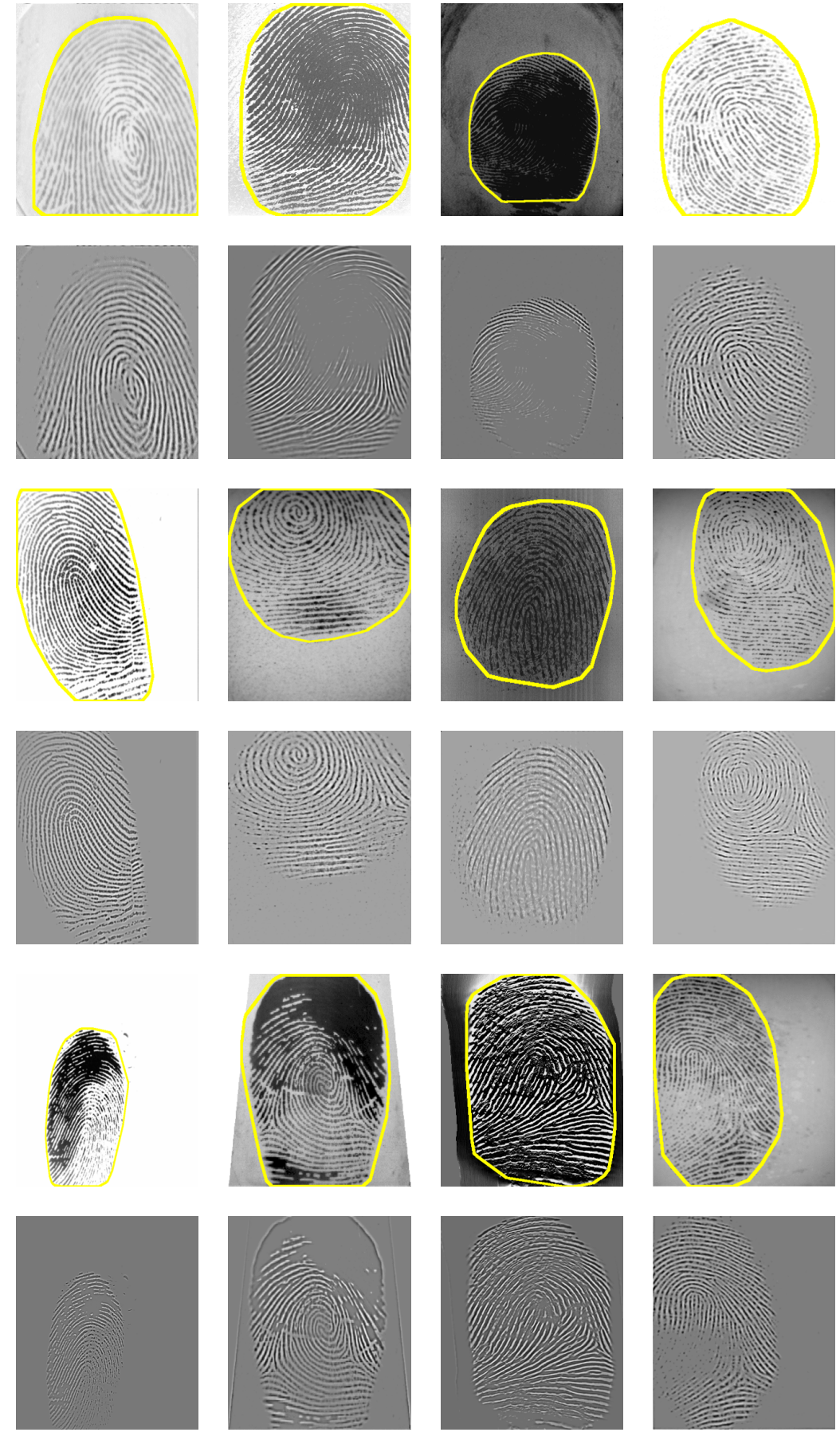

Figure 3.8: Segmented fingerprint images and the corresponding texture images by the variational method for FVC2000 (first and second row), FVC2002 (third and fourth row) and FVC2004 (fifth and sixth row). Columns f.l.t.r correspond to DB1 to DB4. 


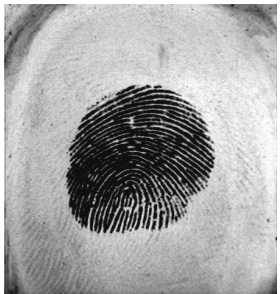

(a)

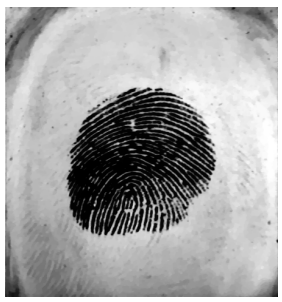

(e) $\lambda_{\mathrm{TV} L_{2}}=0.1$

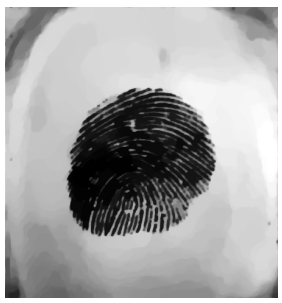

(i) $\lambda_{\mathrm{TV} L_{2}}=0.02$

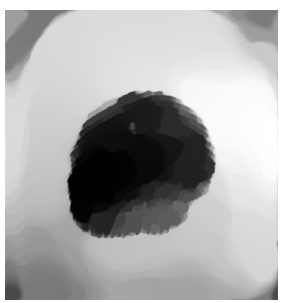

(m) $\lambda_{\mathrm{TV} L_{2}}=0.005$

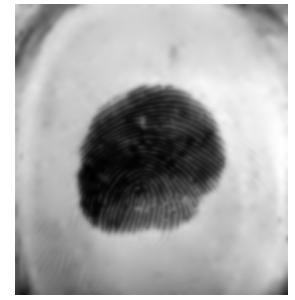

(b) G3PD: $\beta_{1}=$ 0.005

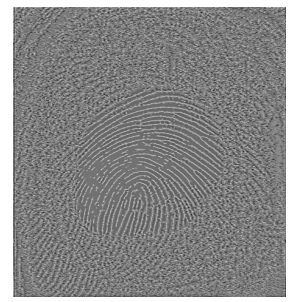

(f)

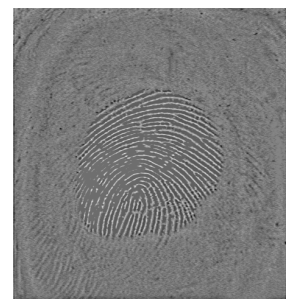

(j)

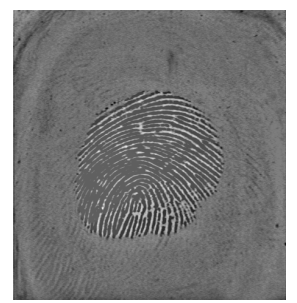

(n)

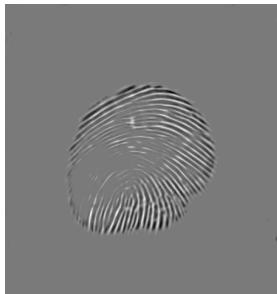

(c)

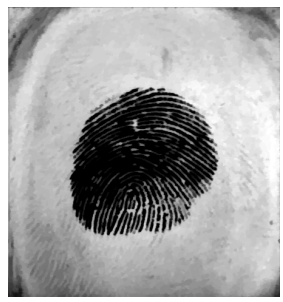

(g) $\lambda_{\mathrm{TV} L_{1}}=1$

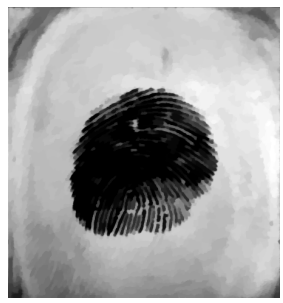

(k) $\lambda_{\mathrm{TV} L_{1}}=0.6$

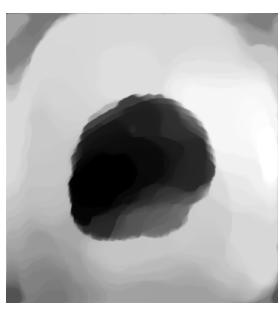

(o) $\lambda_{\mathrm{TV} L_{1}}=0.1$

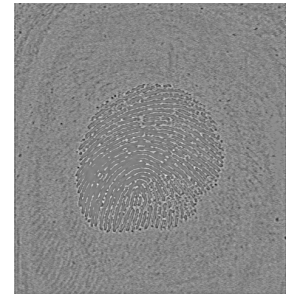

(d)

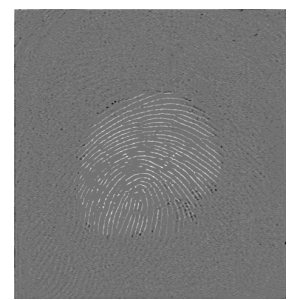

(h)

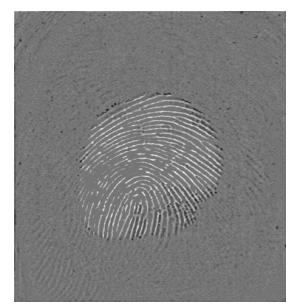

(1)

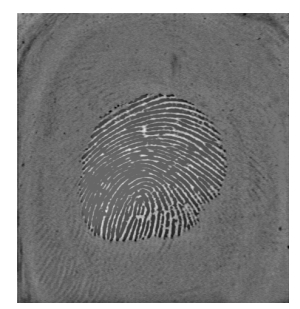

(p)

Figure 3.9: A comparison of G3PD with TV $-L_{2}$ and $\mathrm{TV}-L_{1}$ : First row: images f.l.t.r are the original image $\boldsymbol{f}$ and the three-part decomposition by G3PD with $N=50, \beta_{1}=$ 0.005 (see Table 3.2 for the other parameters): the cartoon image $\boldsymbol{u}$, texture image $\boldsymbol{v}$ and noise image $\boldsymbol{\epsilon}$. The first and second column of rows two to four show images $\boldsymbol{u}$ and $\boldsymbol{v}$, respectively, for TV $-L_{2}$ two-part decomposition with different values of $\lambda_{\mathrm{TV} L_{2}}$. The third and fourth column show the corresponding images $\boldsymbol{u}$ and $\boldsymbol{v}$ for $T V-L 1$ two-part decomposition. The number of iterations for $T V-L_{2}$ and $\mathrm{TV}-L_{1}$ is $N=350$. Note that for no choice of $\lambda_{\mathrm{TV} L_{2}}$ or $\lambda_{\mathrm{TV} L_{1}}$, TV $-L_{2}$ or $\mathrm{TV}-L_{1}$ produce a good feature image for segmentation of this noisy fingerprint, while the G3PD model provides a useful texture image $\boldsymbol{v}$ for the segmentation procedure. 

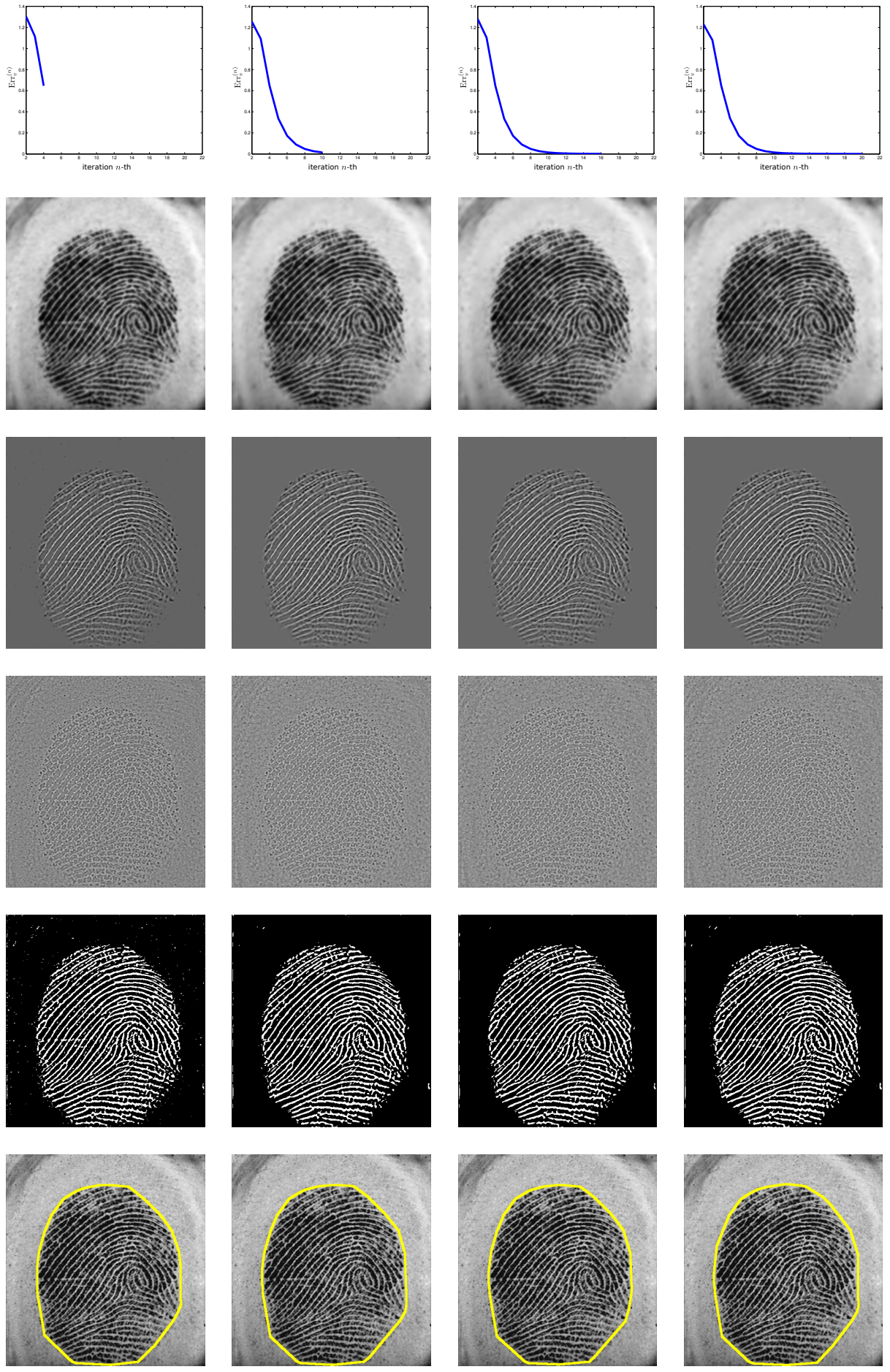

$(\mathrm{u})$ Iter $=4, \operatorname{Err}=(\mathrm{v})$ Iter $=10$, Err $=(\mathrm{w})$ Iter $=16$, Err $=(\mathrm{x})$ Iter $=20$, Err $=$

$$
\begin{array}{llll}
1.51 & 1.53 & 1.61 & 1.69
\end{array}
$$

Figure 3.10: Compare in different iteration with different training parameters for each iteration. Note that the error is lower for 4 iteration (not convergence), perhaps because of the combination with post-processing in segmentation application. 


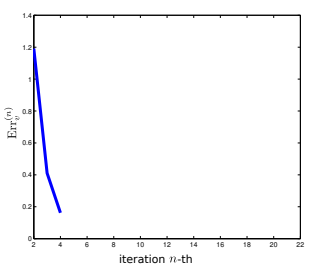

(a)

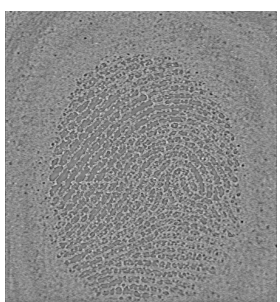

(d)

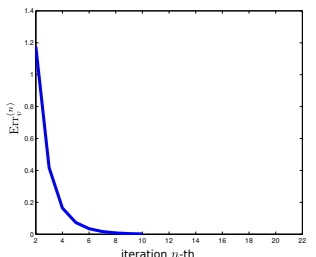

(g)

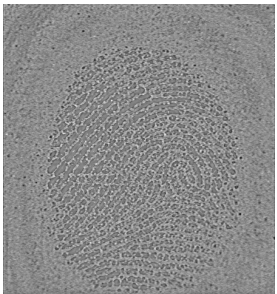

(j)

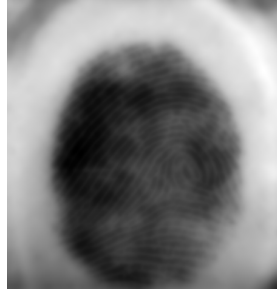

(b)

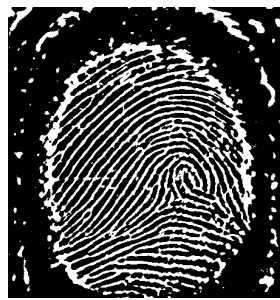

(e)

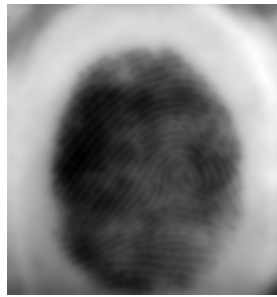

(h)

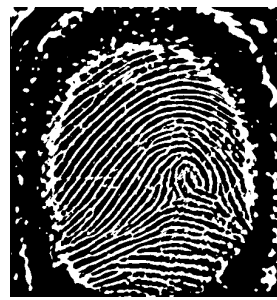

(k)

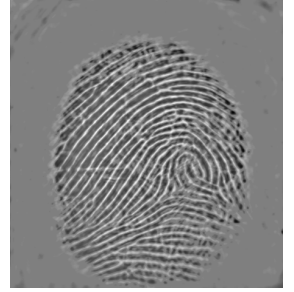

(c)

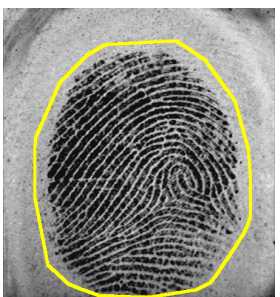

(f)

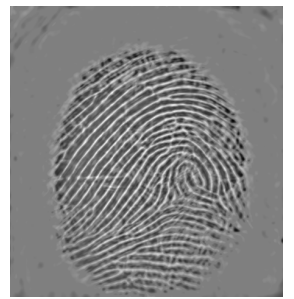

(i)

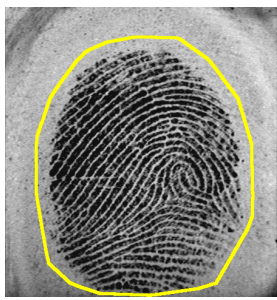

(1)

Figure 3.11: The effect of large $\beta_{1}$ for different iterations. (a)-(f) for 4 iterations with $\beta_{1}=$ $0.06, C=0.055, \beta_{2}=0.001$, Err $=8.09 \%$ : the plot of the convergence of $\boldsymbol{v}$ versus error, the homogeneous region $\boldsymbol{u}$, texture $\boldsymbol{v}$, noise $\boldsymbol{\epsilon}$, binarization of $\boldsymbol{v}$ and the segmented image, respectively. (g)-(l) for 10 iteration with $\beta_{1}=0.06, C=$ $0.045, \beta_{2}=0.001, \operatorname{Err}=9.37 \%$. The large $\beta_{1}$ will generate the halo effect on the boundary of object in $\boldsymbol{v}$ which impact on the segmentation result. In order to reduce the segmentation error due to this effect, we choose a small $\beta_{1}$, e.g. $\beta_{1}=10^{-3}$. So, the homogeneous region $\boldsymbol{u}$ or noise $\boldsymbol{v}$ may contain some texture, but the performance is better. Moreover, we see that the choice of parameters effects severely on the way of decomposition, not the number of iteration. The iteration only guarantees that the solution $(\boldsymbol{u}, \boldsymbol{v}, \boldsymbol{\epsilon})$ is obtained at the convergence with the chosen parameters, if the solution exists. 


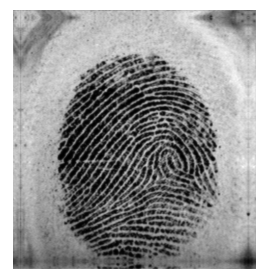

(a) $k=1$
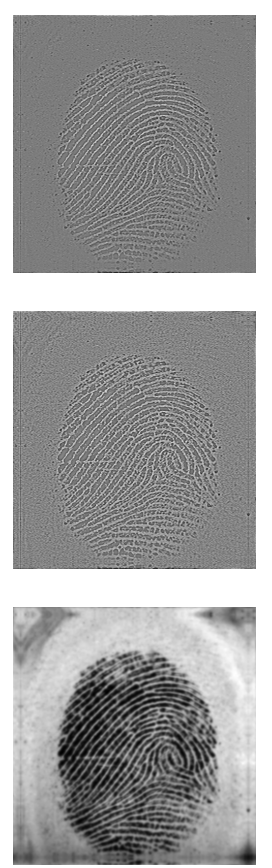

(m) $k=3$
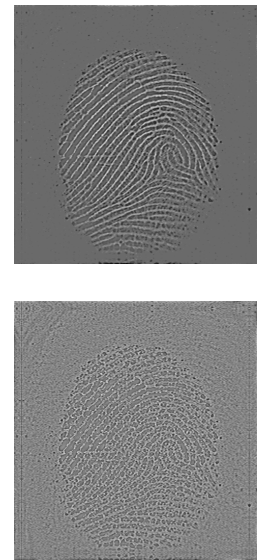
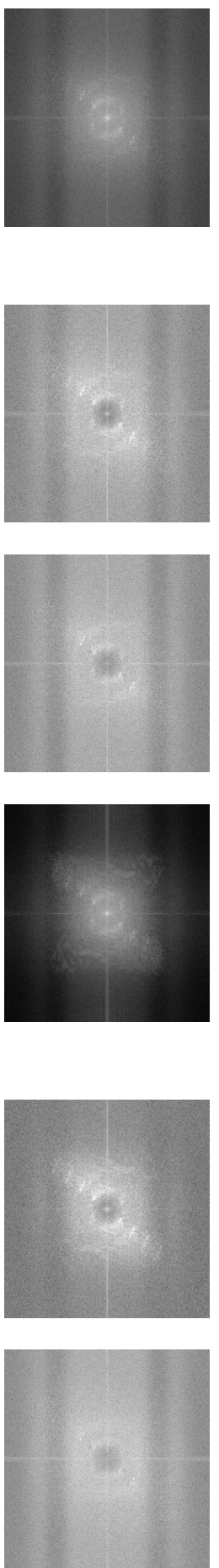

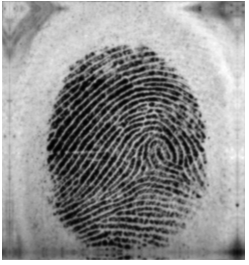

(c) $k=2$
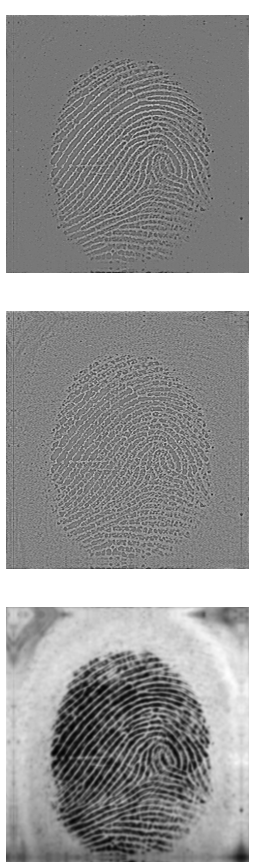

(o) $k=4$
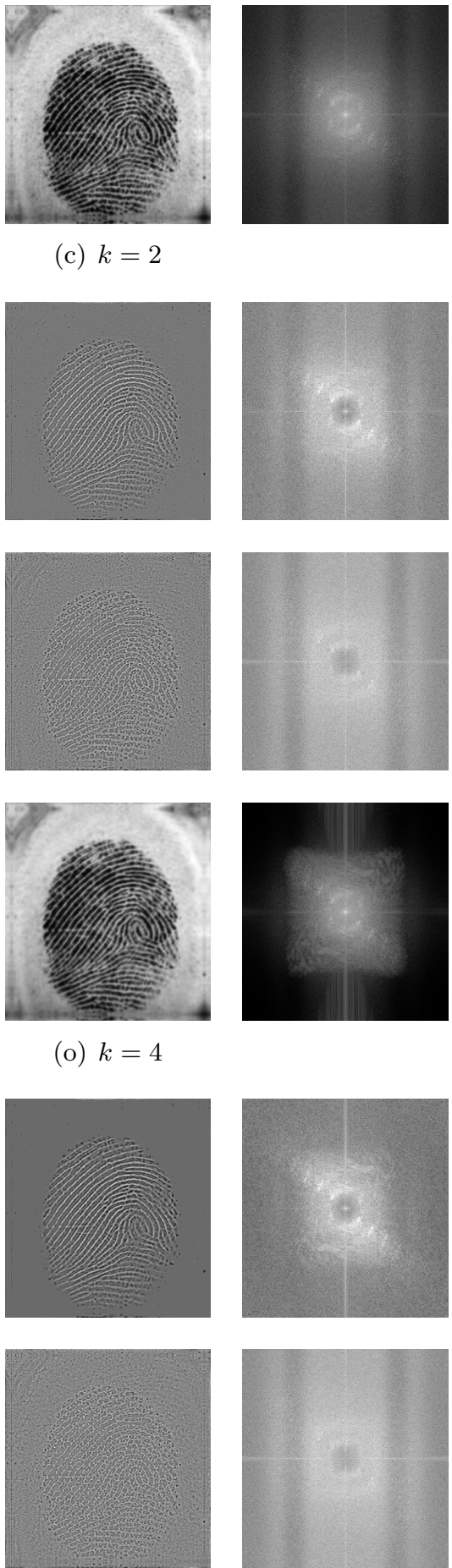

Figure 3.12: The evolution of decomposition of $(\boldsymbol{u}, \boldsymbol{v}, \boldsymbol{\epsilon}$, respectively from top to bottom) with iteration $k=1,2,3,4$ and their Fourier coefficients with $\beta_{1}=0.001$. Row $1-3$ in 1st column is $\boldsymbol{u}, \boldsymbol{v}, \boldsymbol{\epsilon}$ and in 2nd column is their Fourier spectrum at 1st iteration, respectively. Similar for the other pairs with the 2 nd, 3rd and 4 th iterations. 


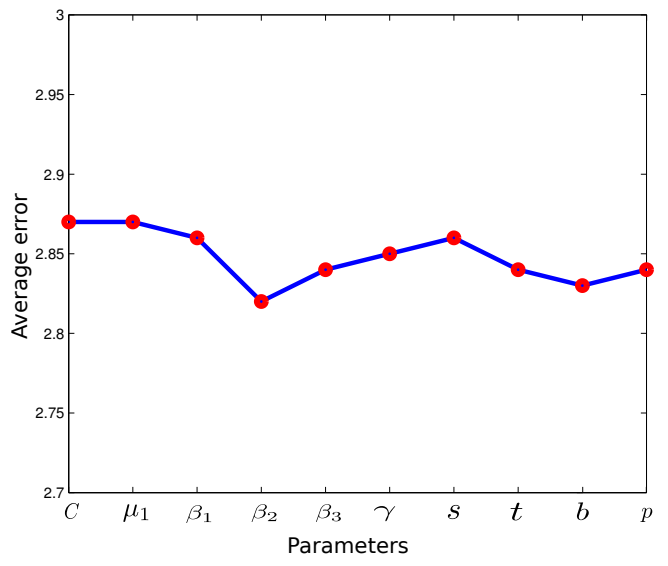

(a)

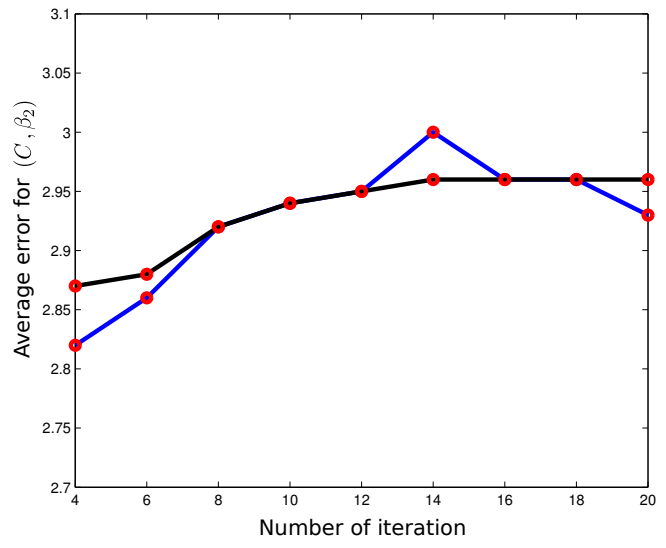

(b)

Figure 3.13: The training procedure: Firstly, $C$ is trained while the other parameters are fixed. Secondly, given the trained $C$, we train the other parameters one by one through different iterations while fixing the rest. Image (a) shows that the minimum of the average error over the training set is obtained at $\beta_{2}$ with $N=4$ iterations. Given different number of iterations, Image (b) depicts that the average error over the training set of $C$ and $\beta_{2}$ is minimum at $N=4$ iteration. 


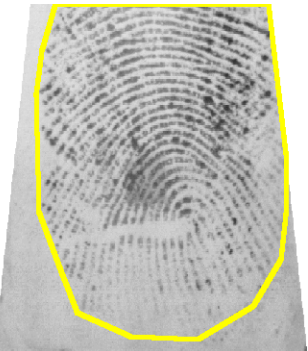

(a)

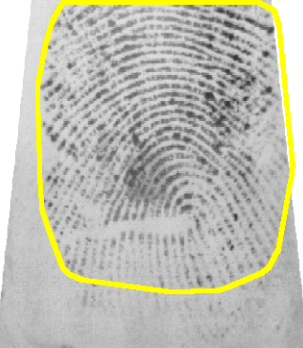

(b) $\operatorname{Err}=8.36$

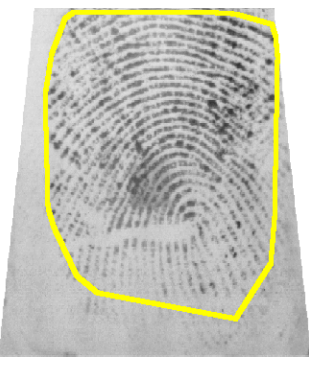

(e) $\operatorname{Err}=10.05$

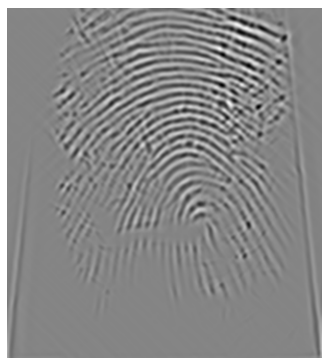

(i)

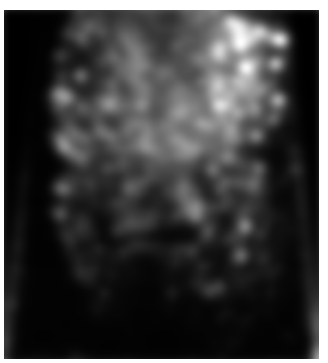

$(\mathrm{m})$

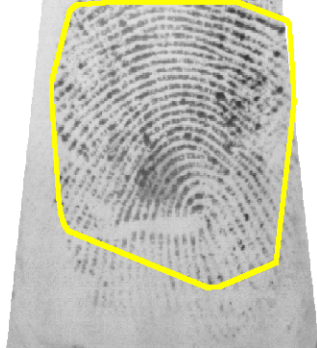

(c) $\operatorname{Err}=14.31$

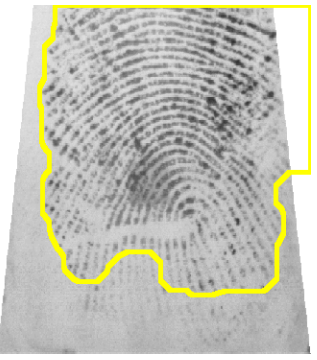

(f) $\operatorname{Err}=14.07$

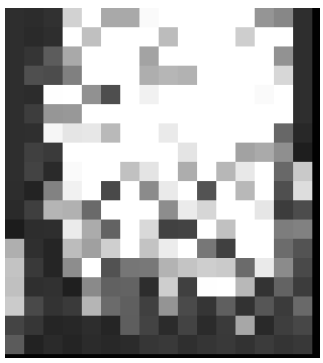

(j)

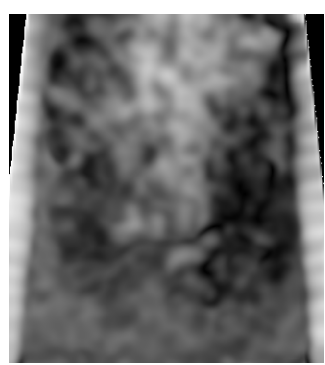

(n)

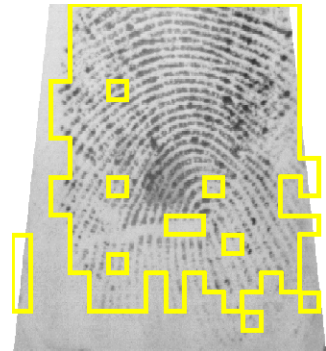

(d) $\operatorname{Err}=13.94$

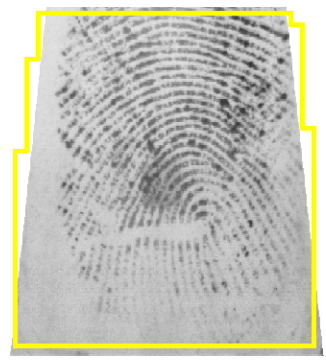

(g) $\operatorname{Err}=22.05$

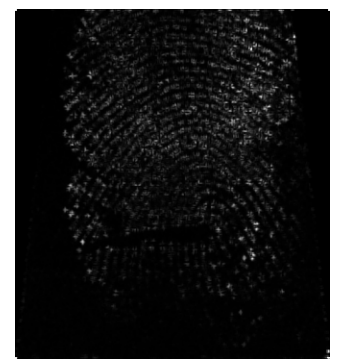

(k)

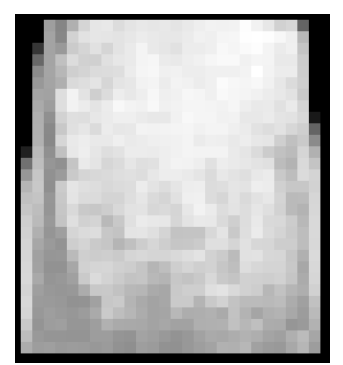

(o)

Figure 3.14: Segmented fingerprint images and their features of different methods for FVC2004_DB2_IM_46_7. (a) ground truth; (b, h) TV , (c, i) FDB, (d, j) Gabor, (e, k) Harris, (f, l, m, n) Mean-Variance-Coherence, (g, o) STFT. 


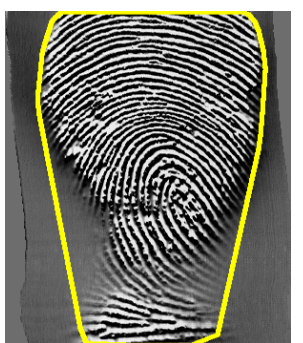

(a) $\operatorname{Err}=6.1$
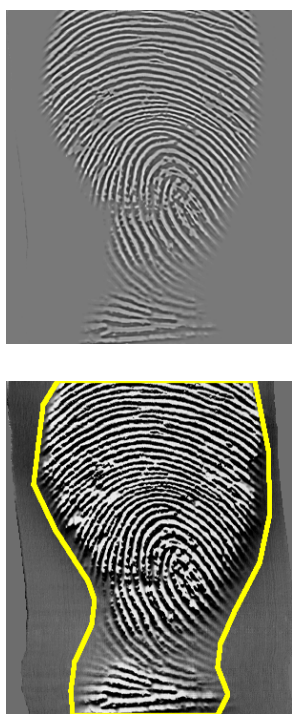

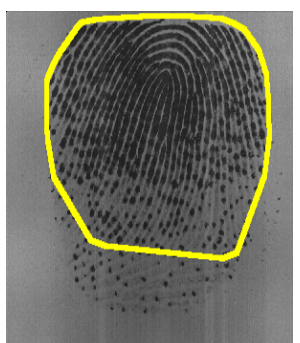

(b) $\operatorname{Err}=12.86$
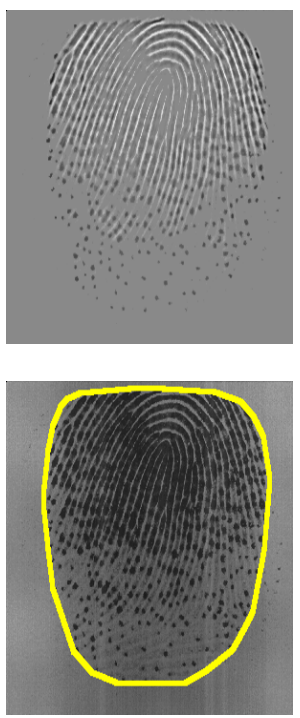

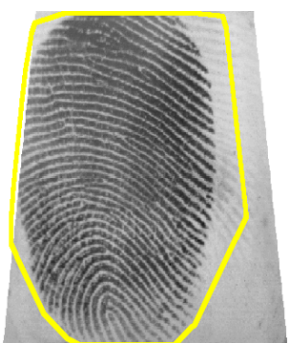

(c) $\operatorname{Err}=9.49$
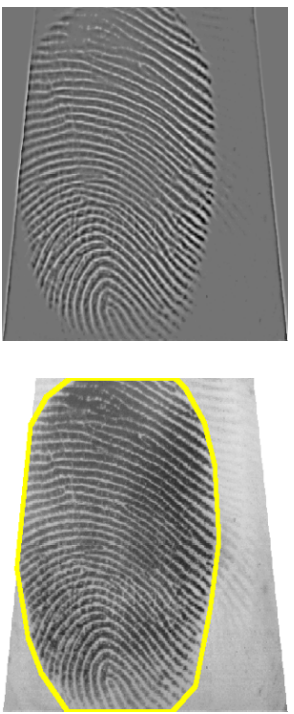

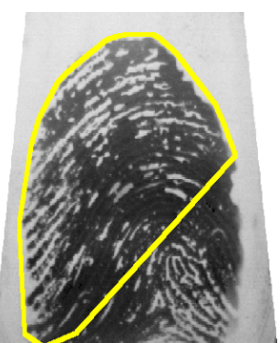

(d) $\operatorname{Err}=18.72$
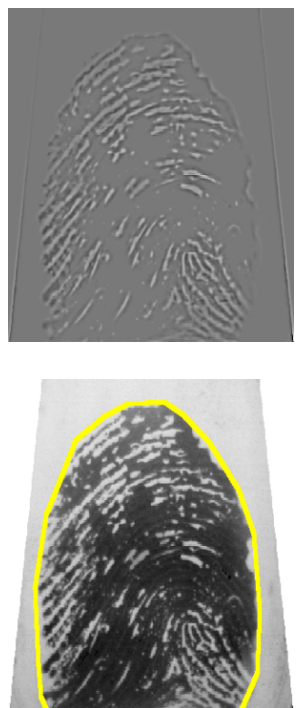

Figure 3.15: Examples of incorrectly segmented fingerprint images due to: (a) the convex hull of the morphological operator, (b) dryness of the finger, (c) a ghost fingerprint on the sensor surface, (d) wetness of the finger. The first row shows the segmentation obtained by the variational method, the second row displays the texture $v$ and the last row visualizes the manually marked ground truth segmentation. 


\section{Supplementary Appendix}

\subsection{Mathematical preliminaries}

\subsubsection{Notation}

For simplification, we use a bold symbol to denote the coordinates of a two-dimensional signal, such as $\boldsymbol{x}=\left(x_{1}, x_{2}\right), \boldsymbol{\omega}=\left(\omega_{1}, \omega_{2}\right), e^{j \boldsymbol{\omega}}=\left[e^{j \omega_{1}}, e^{j \omega_{2}}\right], \boldsymbol{k}=\left[k_{1}, k_{2}\right]$. A twodimensional continuous signal $f(\boldsymbol{x}): \Omega_{c} \rightarrow \mathbb{R}_{+}$is defined on the finite domain with the size $N_{1} \times N_{2}$ :

$$
\Omega_{c}=\left\{\left(x_{1}, x_{2}\right)=\left(N_{1}-1\right) \times\left(N_{2}-1\right) \subset \mathbb{R}_{+}^{2}\right\} .
$$

Whereas, a two-dimensional image $f[\boldsymbol{k}]: \Omega_{d} \rightarrow \mathbb{R}_{+}$, the discretization of $f(\boldsymbol{x})$ (i.e. $\left.f[\boldsymbol{k}]=\left.f(\boldsymbol{x})\right|_{\boldsymbol{x}=\boldsymbol{k} \in \Omega_{d}}\right)$, is specified on the lattice:

$$
\Omega_{d}=\left\{\left(k_{1}, k_{2}\right)=\left\{0, N_{1}-1\right\} \times\left\{0, N_{2}-1\right\} \subset \mathbb{N}^{2}\right\} .
$$

Denote $\boldsymbol{f}=(f[\boldsymbol{k}])_{\boldsymbol{k} \in \Omega_{d}}$ as the vector form of $f[\boldsymbol{k}]$ on the discrete domain $\Omega_{d}$. The discrete finite frequency domain with the coordinates $\boldsymbol{\omega} \in[-\pi, \pi]^{2}$ is determined as

$$
\Omega_{\omega}=\left\{\left(\omega_{1}, \omega_{2}\right)=\left(\frac{2 \pi n_{1}}{N_{1}}, \frac{2 \pi n_{2}}{N_{2}}\right) \mid\left(n_{1}, n_{2}\right)=\left[-\frac{N_{1}}{2}, \frac{N_{1}}{2}\right] \times\left[-\frac{N_{2}}{2}, \frac{N_{2}}{2}\right] \subset \mathbb{N}^{2}\right\} .
$$

Let $X$ be the Euclidean space whose dimension is given by the size of the lattice $\Omega_{d}$, i.e. $X=\mathbb{R}^{\left|\Omega_{d}\right|}$, and $Y=X \times X$ be the vector space. The 2D discrete Fourier transform $\mathcal{F}$ acting on a signal $f[\boldsymbol{k}]$ is expressed as

$$
f[\boldsymbol{k}] \stackrel{\mathcal{F}}{\longleftrightarrow} F\left(e^{j \boldsymbol{\omega}}\right)=\sum_{\boldsymbol{k} \in \Omega_{d}} f[\boldsymbol{k}] \cdot e^{-j\langle\boldsymbol{k}, \boldsymbol{\omega}\rangle_{\ell_{2}}}, \quad \boldsymbol{\omega} \in \Omega_{\omega} .
$$

Let $a, b \in X$, the discrete Hilbert space $\ell_{2}\left(\Omega_{d}\right)$ is equipped with the norm and the inner product:

$$
\begin{aligned}
\|\boldsymbol{a}\|_{\ell_{2}}^{2} & =\sum_{\boldsymbol{k} \in \Omega_{d}} a[\boldsymbol{k}]^{2}, \\
\langle a, b\rangle_{\ell_{2}} & =\sum_{\boldsymbol{k} \in \Omega_{d}} a[\boldsymbol{k}] \cdot b[\boldsymbol{k}] .
\end{aligned}
$$

\subsubsection{Functional Space}

\subsubsection{The discrete bounded variation space $B V\left(\Omega_{d}\right)$}

$$
B V\left(\Omega_{d}\right)=\left\{\boldsymbol{f} \in \ell_{1}\left(\Omega_{d}\right)\left|J(\boldsymbol{f})=\sum_{\boldsymbol{k} \in \Omega_{d}}\right| \nabla f[\boldsymbol{k}] \mid<+\infty\right\} .
$$




\subsubsection{The Besov space $B_{p, q}^{\alpha}$ and curvelet type decomposition space $G_{p, q}^{\alpha}$}

Based on [96], [29] and [97], we give a short brief about the orthogonal wavelet transform and the curvelet transform which correspond to the Besov space and the curvelet type decomposition space, respectively (cf. [94], [107], [62]).

Orthogonal Wavelet Transform The orthogonal basis $\varphi_{i, \boldsymbol{k}}(\boldsymbol{x}) \in L_{2}\left(\Omega_{c}\right)$ at the scale $i^{\text {th }}$ and the translation $\boldsymbol{k}^{\text {th }}$ in $\mathcal{I}=\left\{(i, \boldsymbol{k})=[0, I] \times \Omega_{d} \subset \mathbb{N} \times \mathbb{N}^{2}\right\}$ is

$$
\varphi_{i, \boldsymbol{k}}(\boldsymbol{x})=2^{i / 2} \varphi\left(2^{i} \boldsymbol{x}-\boldsymbol{k}\right), \quad(i, \boldsymbol{k}) \in \mathcal{I},
$$

where $\varphi(\boldsymbol{x})$ is the "mother" wavelet function (see [94]). Since $\varphi_{i, \boldsymbol{k}}(\boldsymbol{x})$ is orthogonal, the linear expansion of a function $f \in L_{2}\left(\Omega_{c}\right)$, i.e.

$$
\begin{aligned}
f(\boldsymbol{x}) & =\sum_{i, \boldsymbol{k}} \underbrace{\left\langle f, \varphi_{i, \boldsymbol{k}}\right\rangle_{L_{2}}}_{\mathcal{W}_{i}\{f\}[\boldsymbol{k}]} \varphi_{i, \boldsymbol{k}}(\boldsymbol{x}) \\
& =\sum_{\boldsymbol{k}}\left\langle f, \varphi_{0, \boldsymbol{k}}\right\rangle_{L_{2}} \varphi_{0, \boldsymbol{k}}(\boldsymbol{x})+\sum_{i \in[1, I], \boldsymbol{k}}\left\langle f, \varphi_{i, \boldsymbol{k}}\right\rangle_{L_{2}} \varphi_{i, \boldsymbol{k}}(\boldsymbol{x}),
\end{aligned}
$$

satisfies a Parseval relation, i.e.

$$
\begin{aligned}
\|f\|_{L_{2}\left(\Omega_{c}\right)}^{2} & =\sum_{i, \boldsymbol{k}}\left|\mathcal{W}_{i}\{f\}[\boldsymbol{k}]\right|^{2} \\
& :=\|\mathcal{W}\{f\}\|_{\ell_{2}\left(\Omega_{d}\right)}^{2}, \quad \text { with } \mathcal{W}\{f\}=\left.\left(\mathcal{W}_{i}\{f\}[\boldsymbol{k}]\right)\right|_{(i, \boldsymbol{k}) \in \mathcal{I}} .
\end{aligned}
$$

Curvelet Transform The frame $\varphi_{i, l, k}(\boldsymbol{x}) \in L_{2}\left(\Omega_{c}\right)$ of the curvelet transform $\mathcal{C}$ at the scale $i$, the orientation $l$ and the translation $\boldsymbol{k}^{\text {th }}$ in

$$
\mathcal{I}=\left\{(i, l, \boldsymbol{k})=[0, I] \times\left[-2^{\lceil i / 2\rceil}, 2^{\lceil i / 2\rceil}-1\right] \times \Omega_{d} \subset \mathbb{N} \times \mathbb{Z} \times \mathbb{N}^{2}\right\},
$$

is

$$
\varphi_{i, l, \boldsymbol{k}}(\boldsymbol{x})=\varphi_{i}\left(R_{\theta_{l}}\left(\boldsymbol{x}-\boldsymbol{x}_{\boldsymbol{k}}^{(i, l)}\right)\right), \quad i, l, \boldsymbol{k} \in \mathcal{I}
$$

where the position is

$$
\boldsymbol{x}_{\boldsymbol{k}}^{(i, l)}=R_{\theta_{l}}^{-1}\left(2^{-i} k_{1}, 2^{-i / 2} k_{2}\right)
$$

the rotation matrix is

$$
R_{\theta}=\left(\begin{array}{cc}
\cos \theta & \sin \theta \\
-\sin \theta & \cos \theta
\end{array}\right), \quad R_{\theta}^{-1}=R_{\theta}^{T}=R_{-\theta},
$$

and $\varphi_{i}(\boldsymbol{x})$ is the "mother" basis function (see [96]). 
The curvelet function $\varphi_{i, l, k}(\boldsymbol{x})$ is a tight frame because the expansion of a function $f \in L_{2}\left(\Omega_{c}\right)$ in the curvelet frame, i.e.

$$
\begin{aligned}
f(\boldsymbol{x}) & =\sum_{i, l, \boldsymbol{k}} \underbrace{\left\langle f, \varphi_{i, l, \boldsymbol{k}}\right\rangle_{L_{2}}}_{\mathcal{C}_{i, l}\{f\}[\boldsymbol{k}]} \varphi_{i, l, \boldsymbol{k}}(\boldsymbol{x}), \\
& =\sum_{\boldsymbol{k}}\left\langle f, \varphi_{0,0, \boldsymbol{k}}\right\rangle_{L_{2}} \varphi_{0,0, \boldsymbol{k}}(\boldsymbol{x})+\sum_{i \in[1, I], l, \boldsymbol{k}}\left\langle f, \varphi_{i, l, \boldsymbol{k}}\right\rangle_{L_{2}} \varphi_{i, l, \boldsymbol{k}}(\boldsymbol{x}),
\end{aligned}
$$

satisfies a Parseval relation, i.e.

$$
\begin{aligned}
\|f\|_{L_{2}\left(\Omega_{c}\right)}^{2} & =\sum_{i, l, \boldsymbol{k}}\left|\mathcal{C}_{i, l}\{f\}[\boldsymbol{k}]\right|^{2} \\
& :=\|\mathcal{C}\{f\}\|_{\ell_{2}\left(\Omega_{d}\right)}^{2}, \quad \text { with } \mathcal{C}\{f\}=\left.\left(\mathcal{C}_{i, l}\{f\}[\boldsymbol{k}]\right)\right|_{(i, l, \boldsymbol{k}) \in \mathcal{I}}
\end{aligned}
$$

In summary, denote $\mathcal{T}$ as the wavelet $\mathcal{W}$ or the curvelet $\mathcal{C}$ transform. The index set is

$$
\mathcal{I}=\left\{(i, l, \boldsymbol{k})=[0, I] \times\left[-2^{\lceil i / 2\rceil}, 2^{\lceil i / 2\rceil}-1\right] \times \Omega_{d} \subset \mathbb{N} \times \mathbb{Z} \times \mathbb{N}^{2}\right\}
$$

Given a function $f(x) \in L_{2}\left(\Omega_{c}\right)$, the $\mathcal{T}$ transform is

$$
f(\boldsymbol{x}) \stackrel{\mathcal{T}}{\longleftrightarrow} \mathcal{T}_{i, l}\{f\}[\boldsymbol{k}], \quad(i, l, \boldsymbol{k}) \in \mathcal{I},
$$

such that the Parseval condition holds, i.e.

$$
\begin{aligned}
\|f\|_{L_{2}\left(\Omega_{c}\right)}^{2} & =\sum_{i, l, \boldsymbol{k}}\left|\mathcal{T}_{i, l}\{f\}[\boldsymbol{k}]\right|^{2} \\
& :=\|\mathcal{T}\{f\}\|_{\ell_{2}\left(\Omega_{d}\right)}^{2}, \quad \text { with } \mathcal{T}\{f\}=\left.\left(\mathcal{T}_{i, l}\{f\}[\boldsymbol{k}]\right)\right|_{(i, l, \boldsymbol{k}) \in \mathcal{I}} .
\end{aligned}
$$

Note that there is no orientation $l$ if $\mathcal{T}$ is the wavelet transform $\mathcal{W}$.

The $B_{p, q}^{\alpha}$ and the $G_{p, q}^{\alpha}$ spaces The Besov space $B_{p, q}^{\alpha}$ relates to orthogonal wavelet coefficients. The curvelet type decomposition space $G_{p, q}^{\alpha}$ corresponds to curvelet coefficients which is more advantage to capture the geometry of the objects.

Denote $A_{p, q}^{\alpha}$ represent for $B_{p, q}^{\alpha}$ or $G_{p, q}^{\alpha}$ with $\alpha>0,0<p, q \leq \infty$. $\mathcal{T}$ is the wavelet $\mathcal{W}$ or the curvelet $\mathcal{C}$ transform of a function $f(x) \in L_{2}\left(\Omega_{c}\right)$ :

$$
f(\boldsymbol{x}) \stackrel{\mathcal{T}}{\longleftrightarrow} \mathcal{T}_{i, l}\{f\}[\boldsymbol{k}], \quad(i, l, \boldsymbol{k}) \in \mathcal{I} .
$$

Let $\mathcal{T}\{f\}=\left.\left(\mathcal{T}_{i, l}\{f\}[\boldsymbol{k}]\right)\right|_{(i, l, \boldsymbol{k}) \in \mathcal{I}}$ be the vector form. 
The space $A_{p, q}^{\alpha}$ is defined as

$$
A_{p, q}^{\alpha}\left(\Omega_{c}\right)=\left\{f \in L_{2}\left(\Omega_{c}\right) \mid\|f\|_{A_{p, q}^{\alpha}}<+\infty\right\}
$$

where the norm is

$$
\|f\|_{A_{p, q}^{\alpha}}=\underbrace{\left(\sum_{\boldsymbol{k}}\left|\mathcal{T}_{0,0}\{f\}[\boldsymbol{k}]\right|^{p}\right)^{\frac{1}{p}}}_{\left\|\mathcal{T}_{0,0}\{f\}\right\|_{\ell_{p}}}+\left(\sum_{l, i \in[1, I]}\left(\sum_{\boldsymbol{k}} w_{i}\left|\mathcal{T}_{i, l}\{f\}[\boldsymbol{k}]\right|^{p}\right)^{\frac{q}{p}}\right)^{\frac{1}{q}},
$$

with the weight

$$
w_{i}=\left\{\begin{array}{ll}
2^{i p\left(\alpha+2\left(\frac{1}{2}-\frac{1}{p}\right)\right)}, & \mathcal{T}=\mathcal{W} \text { (wavelet transform) } \\
2^{i p\left(\alpha+\frac{3}{2}\left(\frac{1}{2}-\frac{1}{p}\right)\right)}, & \mathcal{T}=\mathcal{C} \text { (curvelet transform) }
\end{array} .\right.
$$

If $p=q$, this norm is rewritten as

$$
\begin{aligned}
\|f\|_{A_{p, p}^{\alpha}}^{\alpha} & =\left(\sum_{\boldsymbol{k}}\left|\mathcal{T}_{0,0}\{f\}[\boldsymbol{k}]\right|^{p}\right)^{\frac{1}{p}}+\left(\sum_{l, i \in[1, I]} \sum_{\boldsymbol{k}} w_{i}\left|\mathcal{T}_{i, l}\{f\}[\boldsymbol{k}]\right|^{p}\right)^{\frac{1}{p}} \\
& \asymp\left[\sum_{\boldsymbol{k}}\left|\mathcal{T} f_{0,0}[\boldsymbol{k}]\right|^{p}+\sum_{l, i \in[1, I]} \sum_{\boldsymbol{k}} w_{i}\left|\mathcal{T}_{i, l}\{f\}[\boldsymbol{k}]\right|^{p}\right]^{\frac{1}{p}} \\
& =\left[\sum_{l, i \in[0, I], \boldsymbol{k}} w_{i}\left|\mathcal{T}_{i, l}\{f\}[\boldsymbol{k}]\right|^{p}\right]^{\frac{1}{p}} \\
& :=\|\mathcal{T}\{f\}\|_{w, \ell_{p}}
\end{aligned}
$$

It shows that the norm in the $B_{p, p}^{\alpha}$ and $G_{p, p}^{\alpha}$ spaces is the weighted $\ell_{p}$ norm of the wavelet and curvelet coefficients, respectively, i.e.

$$
\|f\|_{A_{p, p}^{\alpha}} \asymp \underbrace{\left[\sum_{i, l, \boldsymbol{k}} w_{i}\left|\mathcal{T}_{i, l}\{f\}[\boldsymbol{k}]\right|^{p}\right]^{\frac{1}{p}}}_{\|\mathcal{T}\{f\}\|_{w, \ell_{p}}}, \quad(i, l, \boldsymbol{k}) \in \mathcal{I},
$$

Notice that the dual space of $A_{p, q}^{\alpha}$ is $A_{p^{\prime}, q^{\prime}}^{\alpha^{\prime}}$ with satisfying the dual pair

$$
\alpha^{\prime}=-\alpha, p^{\prime}=\frac{p}{p-1} \text { and } q^{\prime}=\frac{q}{q-1} .
$$

Example 3.5.1. Some examples of the norm in the $B_{p, q}^{\alpha}$ or $G_{p, q}^{\alpha}$ spaces with a function $f \in L_{2}\left(\Omega_{c}\right):$ 
- The Besov space with $\alpha=p=q=1$ :

$$
\|f\|_{B_{1,1}^{1}}=\|\mathcal{W}\{f\}\|_{\ell_{1}} .
$$

- The curvelet type decomposition space with $\alpha=\frac{3}{4}, p=q=1$ :

$$
\|f\|_{G_{1,1}^{3 / 4}}=\|\mathcal{C}\{f\}\|_{\ell_{1}} .
$$

- The supremum of the coefficients of $\mathcal{T}$ transform with $q=\infty$

$$
\|f\|_{A_{p, \infty}^{\alpha}}=\left\|\mathcal{T}_{0}\{f\}\right\|_{\ell_{p}}+\sup _{i \in[1, I]}\left\|\mathcal{T}_{i}\{f\}\right\|_{w, \ell_{p}} .
$$

Note that $B_{\infty, \infty}^{-1}$ and $G_{\infty, \infty}^{-3 / 4}$ are the dual space of $B_{1,1}^{1}$ and $G_{1,1}^{3 / 4}$, respectively.

In summary, the space $A_{p, q}^{\alpha}=\left\{B_{p, q}^{\alpha}, G_{p, q}^{\alpha}\right\}$ of the transform $\mathcal{T}=\{\mathcal{W}, \mathcal{C}\}$ with

$$
\mathcal{I}=\left\{(i, l, \boldsymbol{k})=[0, I] \times\left[-2^{\lceil i / 2\rceil}, 2^{\lceil i / 2\rceil}-1\right] \times \Omega_{d} \subset \mathbb{N} \times \mathbb{Z} \times \mathbb{N}^{2}\right\}
$$

is $\quad A_{p, q}^{\alpha}\left(\Omega_{c}\right)=\left\{f \in L_{2}\left(\Omega_{c}\right) \mid\|f\|_{A_{p, q}^{\alpha}}<+\infty\right\}$, and its norm is

$\|f\|_{A_{p, q}^{\alpha}}^{\alpha}=\underbrace{\left(\sum_{\boldsymbol{k}}\left|\mathcal{T}_{0,0}\{f\}[\boldsymbol{k}]\right|^{p}\right)^{\frac{1}{p}}}_{\left\|\mathcal{T}_{0,0}\{f\}\right\|_{\ell_{p}}}+\left(\sum_{l, i \in[1, I]}\left(\sum_{\boldsymbol{k}} w_{i}\left|\mathcal{T}_{i, l}\{f\}[\boldsymbol{k}]\right|^{p}\right)^{\frac{q}{p}}\right)^{\frac{1}{q}}, \quad(i, l, \boldsymbol{k}) \in \mathcal{I}$,

where $w_{i}=\left\{\begin{array}{ll}2^{i p\left(\alpha+2\left(\frac{1}{2}-\frac{1}{p}\right)\right)}, & \mathcal{T}=\mathcal{W} \\ 2^{i p\left(\alpha+\frac{3}{2}\left(\frac{1}{2}-\frac{1}{p}\right)\right)}, & \mathcal{T}=\mathcal{C}\end{array}\right.$.

If $p=q: \quad\|f\|_{A_{p, p}^{\alpha}} \asymp \underbrace{\left[\sum_{i, l, \boldsymbol{k}} w_{i}\left|\mathcal{T}_{i, l}\{f\}[\boldsymbol{k}]\right|^{p}\right]^{\frac{1}{p}}}_{\|\mathcal{T}\{f\}\|_{w, \ell_{p}}}$.

The dual space of $A_{p, q}^{\alpha}$ is $A_{p^{\prime}, q^{\prime}}^{\alpha^{\prime}}$ with $\alpha^{\prime}=-\alpha, p^{\prime}=\frac{p}{p-1}, q^{\prime}=\frac{q}{q-1}$.

\subsubsection{The Legendre Fenchel transform and the Indicator function}

In this section, we give a short outline about how to obtain the indicator function of a closed convex set from the Legendre - Fenchel transform. For more details, we refer the reader to [108] and [109]. 
Definition 3.5.1. The Legendre - Fenchel transform of a convex or nonconvex function $f: U \rightarrow \mathbb{R} \cup\{ \pm \infty\}$ is a convex function $f^{*}: U^{*} \rightarrow \mathbb{R} \cup\{ \pm \infty\}:$

$$
f^{*}\left(\frac{u^{*}}{\mu}\right)=\sup _{u \in U}\left\{\left\langle u, \frac{1}{\mu} u^{*}\right\rangle_{U}-f(u)\right\}, \quad \mu>0 .
$$

Note that the Legendre - Fenchel transformation of $\|\cdot\|_{U}$ in a Banach space $U$ is called "indicator function".

Proposition 3.5.1. Let $\left(U,\|\cdot\|_{U}\right)$ be a real Banach space. Given $\mu>0$, the indicator function of a closed convex set $U(\mu)=\left\{u \in U \mid\|u\|_{U} \leq \mu\right\}$ is

$$
f\left(\frac{u}{\mu}\right)= \begin{cases}0, & u \in U(\mu) \\ +\infty, & u \in U \backslash U(\mu)\end{cases}
$$

Proof. Let $\left(U^{*},\|\cdot\|_{U^{*}}\right)$ be a dual space of a real Banach space $\left(U,\|\cdot\|_{U}\right)$. Given $u \in U$ and $u^{*} \in U^{*}$, the generalized Cauchy Schwarz inequality is

$$
\left\langle u, u^{*}\right\rangle_{U} \leq\|u\|_{U} \cdot\left\|u^{*}\right\|_{U^{*}}
$$

Let $\mu>0$ such that $\left\|u^{*}\right\|_{U^{*}} \leq \mu$. Thus, (3.26) is rewritten as follows

$$
\|u\|_{U} \geq\left\langle u, \frac{1}{\mu} u^{*}\right\rangle_{U}
$$

Let $U^{*}(\mu)=\left\{u^{*} \in U^{*} \mid\left\|u^{*}\right\|_{U^{*}} \leq \mu\right\}$ be a closed convex set. (3.27) is equivalent to

$$
\sup _{u \in U}\left\{\left\langle u, \frac{1}{\mu} u^{*}\right\rangle_{U}-\|u\|_{U}\right\}= \begin{cases}0, & u^{*} \in U^{*}(\mu) \\ +\infty, & u^{*} \in U^{*} \backslash U^{*}(\mu)\end{cases}
$$

Let $f(u)=\|u\|_{U}$. As Definition 3.5.1, the Legendre - Fenchel transform of $F(u)$ is the indicator function:

$$
f^{*}\left(\frac{u^{*}}{\mu}\right)=\left\{\begin{array}{ll}
0, & u^{*} \in U^{*}(\mu) \\
+\infty, & u^{*} \in U^{*} \backslash U^{*}(\mu)
\end{array} .\right.
$$

We rewrite as

$$
f\left(\frac{u}{\mu}\right)=\left\{\begin{array}{ll}
0, & u \in U(\mu) \\
+\infty, & u \in U \backslash U(\mu)
\end{array},\right.
$$

where a closed convex set is $U(\mu)=\left\{u \in U \quad \mid\|u\|_{U} \leq \mu\right\}$. 


\subsection{The propositions and their proofs}

Proposition 3.6.1. The solution of the $\ell_{1}$ minimization problem is the shrinkage operator:

$$
\begin{aligned}
\tilde{\boldsymbol{u}} & =\underset{\boldsymbol{u} \in X}{\operatorname{argmin}}\left\{\|\boldsymbol{u}\|_{\ell_{1}}+\frac{\alpha}{2}\|\boldsymbol{f}-\boldsymbol{u}\|_{\ell_{2}}^{2}\right\} \\
& =\frac{\boldsymbol{f}}{|\boldsymbol{f}|} \cdot \max \left(|\boldsymbol{f}|-\frac{1}{\alpha}, 0\right) \\
& :=\operatorname{Shrink}\left(\boldsymbol{f}, \frac{1}{\alpha}\right) .
\end{aligned}
$$

Proof. The minimization is rewritten as follows

$$
\tilde{\boldsymbol{u}}=\underset{\boldsymbol{u} \in X}{\operatorname{argmin}}\left\{\sum_{\boldsymbol{k} \in \Omega_{d}}|u[\boldsymbol{k}]|+\frac{\alpha}{2} \sum_{\boldsymbol{k} \in \Omega_{d}}(f[\boldsymbol{k}]-u[\boldsymbol{k}])^{2}\right\}
$$

The minimization reduces to $|\Omega|$ scalar minimizations, separately:

$$
\tilde{u}[\boldsymbol{k}]=\underset{u[\boldsymbol{k}] \in \mathbb{R}}{\operatorname{argmin}}\left\{|u[\boldsymbol{k}]|+\frac{\alpha}{2}(f[\boldsymbol{k}]-u[\boldsymbol{k}])^{2}\right\}
$$

The solution $\tilde{u}[\boldsymbol{k}]$ is the discrete version of the continuous minimization:

$$
\tilde{u}(\boldsymbol{x})=\underset{u(\boldsymbol{x}) \in \mathbb{R}}{\operatorname{argmin}}\{\underbrace{|u(\boldsymbol{x})|+\frac{\alpha}{2}(f(\boldsymbol{x})-u(\boldsymbol{x}))^{2}}_{:=F(\boldsymbol{x}, u(\boldsymbol{x}))}\}
$$

$F: \Omega_{d} \times \mathbb{R} \rightarrow \mathbb{R} \cup\{ \pm \infty\}$ is a non-differentiable convex function. According to Theorem 4 [108, p. 247], the minimizer is obtained by $0 \in \frac{\partial F(\boldsymbol{x}, u(\boldsymbol{x}))}{\partial u(\boldsymbol{x})}$ :

$$
\begin{aligned}
0 & = \begin{cases}1+\alpha(u(\boldsymbol{x})-f(\boldsymbol{x})), & u(\boldsymbol{x})>0 \\
-1+\alpha(u(\boldsymbol{x})-f(\boldsymbol{x})), & u(\boldsymbol{x})<0 \\
\nu+\alpha(u(\boldsymbol{x})-f(\boldsymbol{x})), & u(\boldsymbol{x})=0 \text { and } \nu \in[-1,1]\end{cases} \\
\Leftrightarrow \tilde{u}(\boldsymbol{x}) & = \begin{cases}f(\boldsymbol{x})-\frac{1}{\alpha}, & f(\boldsymbol{x})>\frac{1}{\alpha}>0 \\
f(\boldsymbol{x})+\frac{1}{\alpha}, & f(\boldsymbol{x})<-\frac{1}{\alpha}<0 \\
0, & f(\boldsymbol{x}) \in\left[-\frac{1}{\alpha}, \frac{1}{\alpha}\right]\end{cases} \\
& = \begin{cases}\frac{f(\boldsymbol{x})}{|f(\boldsymbol{x})|} \cdot\left(|f(\boldsymbol{x})|-\frac{1}{\alpha}\right), & f(\boldsymbol{x})<-\frac{1}{\alpha} \text { or } f(\boldsymbol{x})>\frac{1}{\alpha} \\
0, & f(\boldsymbol{x}) \in\left[-\frac{1}{\alpha}, \frac{1}{\alpha}\right]\end{cases} \\
= & \frac{f(\boldsymbol{x})}{|f(\boldsymbol{x})|} \cdot \max \left(|f(\boldsymbol{x})|-\frac{1}{\alpha}, 0\right) .
\end{aligned}
$$


The discrete version $\tilde{u}[\boldsymbol{k}]=\left.\tilde{u}(\boldsymbol{x})\right|_{\boldsymbol{x}=\boldsymbol{k} \in \Omega_{d}}$ is

$$
\tilde{u}[\boldsymbol{k}]=\frac{f[\boldsymbol{k}]}{|f[\boldsymbol{k}]|} \cdot \max \left(|f[\boldsymbol{k}]|-\frac{1}{\alpha}, 0\right)
$$

The vector form of $\left.\tilde{u}[\boldsymbol{k}]\right|_{\boldsymbol{k} \in \Omega_{d}}$ with the point-wise operators $(-, \times, /, \max ,|\cdot|)$ is

$$
\begin{aligned}
\tilde{\boldsymbol{u}} & =\frac{\boldsymbol{f}}{|\boldsymbol{f}|} \cdot \max \left(|\boldsymbol{f}|-\frac{1}{\alpha}, 0\right) \\
& :=\operatorname{Shrink}\left(\boldsymbol{f}, \frac{1}{\alpha}\right),
\end{aligned}
$$

Proposition 3.6.2. The solution of the regularization in the curvelet type decomposition space $G_{1,1}^{3 / 4}$ is the curvelet soft thresholding operator:

$$
\begin{aligned}
\tilde{\boldsymbol{u}} & =\underset{\boldsymbol{u} \in X}{\operatorname{argmin}}\left\{\|\boldsymbol{u}\|_{G_{1,1}^{3 / 4}}+\frac{1}{2 \delta}\|\boldsymbol{f}-\boldsymbol{u}\|_{\ell_{2}}^{2}\right\} \\
& =\mathcal{C}^{*}\{\operatorname{Shrink}(\mathcal{C}\{\boldsymbol{f}\}, \delta)\} \\
& :=\operatorname{CST}(\boldsymbol{f}, \delta) .
\end{aligned}
$$

Proof. Instead of the Besov space in [94], this proof is for the curvelet type decomposition space $G_{1,1}^{3 / 4}$. As mentioned before, the norm of a function belongs to $G_{p, q}^{\alpha}$ is the weighted $\ell_{p}$ norm of the curvelet coefficients; especially with $G_{1,1}^{3 / 4}$, it is equivalent to the $\ell_{1}$ norm of the curvelet coefficients, i.e.

$$
\|\boldsymbol{u}\|_{G_{1,1}^{3 / 4}}=\|\mathcal{C}\{\boldsymbol{u}\}\|_{\ell_{1}},
$$

and the curvelet transform is the unitary operator on $\ell_{2}\left(\Omega_{d}\right)$, i.e.

$$
\|\boldsymbol{f}-\boldsymbol{u}\|_{\ell_{2}\left(\Omega_{d}\right)}=\|\mathcal{C}\{\boldsymbol{f}\}-\mathcal{C}\{\boldsymbol{u}\}\|_{\ell_{2}\left(\Omega_{d}\right)}, \quad \forall f, u \in \ell_{2}\left(\Omega_{d}\right) .
$$

Thus, the minimization is reformulated as follows

$$
\tilde{\boldsymbol{u}}=\underset{\boldsymbol{u} \in X}{\operatorname{argmin}}\left\{\|\mathcal{C}\{\boldsymbol{u}\}\|_{\ell_{1}}+\frac{1}{2 \delta}\|\mathcal{C}\{\boldsymbol{f}\}-\mathcal{C}\{\boldsymbol{u}\}\|_{\ell_{2}}^{2}\right\}
$$

Let $\boldsymbol{t}=\mathcal{C}\{\boldsymbol{u}\}$, we have

$$
\tilde{\boldsymbol{t}}=\underset{\boldsymbol{t}}{\operatorname{argmin}}\left\{\|\boldsymbol{t}\|_{\ell_{1}}+\frac{1}{2 \delta}\|\mathcal{C}\{\boldsymbol{f}\}-\boldsymbol{t}\|_{\ell_{2}}^{2}\right\}
$$

As in Proposition 3.6.1, the solution of this $\ell_{1}$ minimization is

$$
\tilde{\boldsymbol{t}}=\operatorname{Shrink}(\mathcal{C}\{\boldsymbol{f}\}, \delta) .
$$


Thus, the solution in the vector form is obtained by the inverse curvelet transform as follows

$$
\begin{gathered}
\tilde{\boldsymbol{u}}=\mathcal{C}^{*}\{\tilde{\boldsymbol{t}}\}=\mathcal{C}^{*}\{\operatorname{Shrink}(\mathcal{C}\{\boldsymbol{f}\}, \delta)\} \\
:=\operatorname{CST}(\boldsymbol{f}, \delta), \quad \forall \boldsymbol{u}, \boldsymbol{f} \in \ell_{2}\left(\Omega_{d}\right) .
\end{gathered}
$$

Proposition 3.6.3. The solution of the "v-problem":

$$
\min _{\boldsymbol{v} \in X}\left\{\mu_{2}\|\boldsymbol{v}\|_{\ell_{1}}+\frac{\beta_{2}}{2}\left\|\boldsymbol{w}-\mathcal{C}\{\boldsymbol{v}\}+\frac{\boldsymbol{\lambda}_{\mathbf{2}}}{\beta_{2}}\right\|_{\ell_{2}}^{2}+\frac{\beta_{3}}{2}\left\|\boldsymbol{f}-\boldsymbol{u}-\boldsymbol{v}-\boldsymbol{\epsilon}+\frac{\boldsymbol{\lambda}_{\mathbf{3}}}{\beta_{3}}\right\|_{\ell_{2}}^{2}\right\}
$$

is

$$
\tilde{\boldsymbol{v}}=\operatorname{Shrink}\left(\boldsymbol{A}, \frac{\mu_{2}}{\beta_{2}+\beta_{3}}\right) \quad \text { with } \quad \boldsymbol{A}=\frac{\mathcal{C}^{*}\left\{\beta_{2} \boldsymbol{w}+\boldsymbol{\lambda}_{\mathbf{2}}\right\}+\beta_{3}\left(\boldsymbol{f}-\boldsymbol{u}-\boldsymbol{\epsilon}+\frac{\boldsymbol{\lambda}_{\mathbf{3}}}{\beta_{3}}\right)}{\beta_{2}+\beta_{3}} .
$$

Proof. Due to the curvelet transform $\mathcal{C}$ is unitary on $\ell_{2}\left(\Omega_{d}\right)$, i.e.

$$
\left\|\boldsymbol{w}-\mathcal{C}\{\boldsymbol{v}\}+\frac{\boldsymbol{\lambda}_{\mathbf{2}}}{\beta_{2}}\right\|_{\ell_{2}\left(\Omega_{d}\right)}=\left\|\mathcal{C}^{*}\{\boldsymbol{w}\}-\boldsymbol{v}+\frac{\mathcal{C}^{*}\left\{\boldsymbol{\lambda}_{\mathbf{2}}\right\}}{\beta_{2}}\right\|_{\ell_{2}\left(\Omega_{d}\right)},
$$

and the separability property of the pixels, the minimization reduces to performing separately $\Omega_{d}$ scalar minimizations with $\boldsymbol{k} \in \Omega_{d}$ :

$$
\begin{aligned}
\tilde{v}[\boldsymbol{k}]=\underset{v[\boldsymbol{k}] \in \mathbb{R}}{\operatorname{argmin}}\left\{\mu_{2}|v[\boldsymbol{k}]|\right. & +\frac{\beta_{2}}{2}\left(\mathcal{C}^{*}\{w\}[\boldsymbol{k}]-v[\boldsymbol{k}]+\frac{\mathcal{C}^{*}\left\{\lambda_{2}\right\}[\boldsymbol{k}]}{\beta_{2}}\right)^{2} \\
& \left.+\frac{\beta_{3}}{2}\left(f[\boldsymbol{k}]-u[\boldsymbol{k}]-v[\boldsymbol{k}]-\epsilon[\boldsymbol{k}]+\frac{\lambda_{3}[\boldsymbol{k}]}{\beta_{3}}\right)^{2}\right\}
\end{aligned}
$$

The solution of this minimization is the discrete version of the continuous minimization:

$$
\begin{aligned}
\tilde{v}(\boldsymbol{x})=\underset{v(\boldsymbol{x}) \in \mathbb{R}}{\operatorname{argmin}}\left\{F(\boldsymbol{x}, v(\boldsymbol{x})):=\mu_{2}|v(\boldsymbol{x})|\right. & +\frac{\beta_{2}}{2}\left(\mathcal{C}^{*}\{w\}(\boldsymbol{x})-v(\boldsymbol{x})+\frac{\mathcal{C}^{*}\left\{\lambda_{2}\right\}(\boldsymbol{x})}{\beta_{2}}\right)^{2} \\
& \left.+\frac{\beta_{3}}{2}\left(f(\boldsymbol{x})-u(\boldsymbol{x})-v(\boldsymbol{x})-\epsilon(\boldsymbol{x})+\frac{\lambda_{3}(\boldsymbol{x})}{\beta_{3}}\right)^{2}\right\}
\end{aligned}
$$

where $F: \Omega_{c} \times \mathbb{R} \rightarrow \mathbb{R} \cup\{ \pm \infty\}$ is the non-differentiable convex function. The subdif- 
ferential at the optimality, i.e. $0 \in \frac{\partial F(\boldsymbol{x}, v(\boldsymbol{x}))}{\partial v(\boldsymbol{x})}$, is

$$
\begin{aligned}
\Leftrightarrow 0 & =\mu_{2} \frac{v(\boldsymbol{x})}{|v(\boldsymbol{x})|}-\beta_{2} \mathcal{C}^{*}\left\{w+\frac{\lambda_{2}}{\beta_{2}}\right\}(\boldsymbol{x})+\beta_{2} v(\boldsymbol{x})-\beta_{3}\left(f(\boldsymbol{x})-u(\boldsymbol{x})-v(\boldsymbol{x})-\epsilon(\boldsymbol{x})+\frac{\lambda_{3}(\boldsymbol{x})}{\beta_{3}}\right) \\
\Leftrightarrow \tilde{v}(\boldsymbol{x}) & =\frac{\beta_{2}}{\beta_{2}+\beta_{3}} \mathcal{C}^{*}\left\{w+\frac{\lambda_{2}}{\beta_{2}}\right\}(\boldsymbol{x})+\frac{\beta_{3}}{\beta_{2}+\beta_{3}}\left(f(\boldsymbol{x})-u(\boldsymbol{x})-\epsilon(\boldsymbol{x})+\frac{\lambda_{3}(\boldsymbol{x})}{\beta_{3}}\right)-\frac{\mu_{2}}{\beta_{2}+\beta_{3}} \frac{v(\boldsymbol{x})}{|v(\boldsymbol{x})|} \\
& :=\operatorname{Shrink}(\underbrace{\frac{\beta_{2}}{\beta_{2}+\beta_{3}} \mathcal{C}^{*}\left\{w+\frac{\lambda_{2}}{\beta_{2}}\right\}(\boldsymbol{x})+\frac{\beta_{3}}{\beta_{2}+\beta_{3}}\left(f(\boldsymbol{x})-u(\boldsymbol{x})-\epsilon(\boldsymbol{x})+\frac{\lambda_{3}(\boldsymbol{x})}{\beta_{3}}\right)}_{:=A(x)}, \frac{\mu_{2}}{\beta_{2}+\beta_{3}}) .
\end{aligned}
$$

The discrete version $\tilde{v}[\boldsymbol{k}]=\left.\tilde{v}(\boldsymbol{x})\right|_{\boldsymbol{x}=\boldsymbol{k} \in \Omega_{d}}$ is

$$
\tilde{v}[\boldsymbol{k}]=\operatorname{Shrink}\left(A[\boldsymbol{k}], \frac{\mu_{2}}{\beta_{2}+\beta_{3}}\right) .
$$

Finally, we obtain the vector form of $\left.\tilde{v}[\boldsymbol{k}]\right|_{\boldsymbol{k} \in \Omega_{d}}$ with the component-wise operators $(+,-, \times, /)$ :

$$
\tilde{\boldsymbol{v}}=\operatorname{Shrink}\left(\boldsymbol{A}, \frac{\mu_{2}}{\beta_{2}+\beta_{3}}\right)
$$

with

$$
\boldsymbol{A}=\frac{\beta_{2}}{\beta_{2}+\beta_{3}} \mathcal{C}^{*}\left\{\boldsymbol{w}+\frac{\boldsymbol{\lambda}_{\mathbf{2}}}{\beta_{2}}\right\}+\frac{\beta_{3}}{\beta_{2}+\beta_{3}}\left(\boldsymbol{f}-\boldsymbol{u}-\boldsymbol{\epsilon}+\frac{\boldsymbol{\lambda}_{\mathbf{3}}}{\beta_{3}}\right)
$$

Proposition 3.6.4. The solution of the " $\epsilon$-problem" is as follows

$$
\begin{aligned}
\tilde{\boldsymbol{\epsilon}} & =\underset{\boldsymbol{\epsilon} \in X}{\operatorname{argmin}}\left\{G^{*}\left(\frac{\boldsymbol{\epsilon}}{\delta}\right)+\frac{\beta_{3}}{2}\left\|\boldsymbol{\epsilon}-\left(\boldsymbol{f}-\boldsymbol{u}-\boldsymbol{v}+\frac{\boldsymbol{\lambda}_{\mathbf{3}}}{\beta_{3}}\right)\right\|_{\ell_{2}}^{2}\right\} \\
& =\left(\boldsymbol{f}-\boldsymbol{u}-\boldsymbol{v}+\frac{\boldsymbol{\lambda}_{\mathbf{3}}}{\beta_{3}}\right)-\operatorname{CST}\left(\boldsymbol{f}-\boldsymbol{u}-\boldsymbol{v}+\frac{\boldsymbol{\lambda}_{\mathbf{3}}}{\beta_{3}}, \delta\right) .
\end{aligned}
$$

Proof. The proof for the " $\epsilon$-problem" is based on [101] with the extension to the curvelet type decomposition space instead of the Besov space. The minimization is reformulated as

$$
\begin{aligned}
\tilde{\boldsymbol{\epsilon}} & =\underset{\boldsymbol{\epsilon} \in S(\delta)}{\operatorname{argmin}}\left\{\left\|\boldsymbol{\epsilon}-\left(\boldsymbol{f}-\boldsymbol{u}-\boldsymbol{v}+\frac{\boldsymbol{\lambda}_{\mathbf{3}}}{\beta_{3}}\right)\right\|_{\ell_{2}}^{2}\right\} \\
& =P_{S(\delta)}\left(\boldsymbol{f}-\boldsymbol{u}-\boldsymbol{v}+\frac{\boldsymbol{\lambda}_{\mathbf{3}}}{\beta_{3}}\right)
\end{aligned}
$$


To compute this projection, the dual problem is considered instead

$$
\tilde{\boldsymbol{\epsilon}}_{\boldsymbol{d}}=\underset{\boldsymbol{\epsilon}_{\boldsymbol{d}} \in X}{\operatorname{argmin}}\left\{\delta\left\|\boldsymbol{\epsilon}_{\boldsymbol{d}}\right\|_{G_{1,1}^{3 / 4}}+\frac{1}{2}\left\|\boldsymbol{\epsilon}_{\boldsymbol{d}}-\left(\boldsymbol{f}-\boldsymbol{u}-\boldsymbol{v}+\frac{\boldsymbol{\lambda}_{\mathbf{3}}}{\beta_{3}}\right)\right\|_{\ell_{2}}^{2}\right\}
$$

According to the Proposition 3.6.2, the solution of the above minimization for the curvelet decomposition space is the curvelet soft thresholding as follows

$$
\begin{aligned}
\tilde{\boldsymbol{\epsilon}}_{\boldsymbol{d}} & =\mathcal{C}^{*}\left\{\operatorname{Shrink}\left(\mathcal{C}\left\{\boldsymbol{f}-\boldsymbol{u}-\boldsymbol{v}+\frac{\boldsymbol{\lambda}_{\mathbf{3}}}{\beta_{3}}\right\}, \delta\right)\right\} \\
& :=\operatorname{CST}\left(\boldsymbol{f}-\boldsymbol{u}-\boldsymbol{v}+\frac{\boldsymbol{\lambda}_{\mathbf{3}}}{\beta_{3}}, \delta\right) .
\end{aligned}
$$

From Proposition 4.7 (see [101]), the solution of the primal problem is

$$
\begin{aligned}
\tilde{\boldsymbol{\epsilon}} & =\left(\boldsymbol{f}-\boldsymbol{u}-\boldsymbol{v}+\frac{\boldsymbol{\lambda}_{\mathbf{3}}}{\beta_{3}}\right)-\tilde{\boldsymbol{\epsilon}}_{\boldsymbol{d}} \\
& =\left(\boldsymbol{f}-\boldsymbol{u}-\boldsymbol{v}+\frac{\boldsymbol{\lambda}_{\mathbf{3}}}{\beta_{3}}\right)-\operatorname{CST}\left(\boldsymbol{f}-\boldsymbol{u}-\boldsymbol{v}+\frac{\boldsymbol{\lambda}_{\mathbf{3}}}{\beta_{3}}, \delta\right) .
\end{aligned}
$$

Proposition 3.6.5. The solution of the "u-problem" is as follows

$$
\begin{aligned}
\tilde{\boldsymbol{u}} & =\underset{\boldsymbol{u} \in X}{\operatorname{argmin}}\left\{\frac{\beta_{1}}{2}\left\|\boldsymbol{p}-\nabla_{d} \boldsymbol{u}+\frac{\boldsymbol{\lambda}_{\mathbf{1}}}{\beta_{1}}\right\|_{\ell_{2}}^{2}+\frac{\beta_{3}}{2}\left\|\boldsymbol{f}-\boldsymbol{u}-\boldsymbol{v}-\boldsymbol{\epsilon}+\frac{\boldsymbol{\lambda}_{\mathbf{3}}}{\beta_{3}}\right\|_{\ell_{2}}^{2}\right\}, \\
& =\operatorname{Re}\left[\mathcal{F}^{-1}\left\{\frac{D\left(e^{j \boldsymbol{\omega}}\right)}{\beta_{3}+4 \beta_{1}\left[\sin ^{2}\left(\frac{\omega_{1}}{2}\right)+\sin ^{2}\left(\frac{\omega_{2}}{2}\right)\right]}\right\}\right][\boldsymbol{k}], \quad \forall \boldsymbol{k} \in \Omega_{d} \text { and } \boldsymbol{\omega} \in \Omega_{\boldsymbol{\omega}},
\end{aligned}
$$

Proof. Due to the separability property of the pixels, the minimization with $\boldsymbol{k} \in \Omega_{d}$ reduces to $\left|\Omega_{d}\right|$ scalar minimizations as

$$
\tilde{u}[\boldsymbol{k}]=\underset{u[\boldsymbol{k}] \in \mathbb{R}}{\operatorname{argmin}}\left\{\frac{\beta_{1}}{2}\left|p[\boldsymbol{k}]-\nabla_{\mathrm{d}} u[\boldsymbol{k}]+\frac{\lambda_{1}[\boldsymbol{k}]}{\beta_{1}}\right|^{2}+\frac{\beta_{3}}{2}\left(f[\boldsymbol{k}]-u[\boldsymbol{k}]-v[\boldsymbol{k}]-\epsilon[\boldsymbol{k}]+\frac{\lambda_{3}[\boldsymbol{k}]}{\beta_{3}}\right)^{2}\right\} .
$$

The solution $\tilde{u}[\boldsymbol{k}]$ is the discrete version of the continuous minimization with the continuous gradient operator $\nabla u(\boldsymbol{x})=\left(\frac{\partial u(\boldsymbol{x})}{\partial x_{1}}, \frac{\partial u(\boldsymbol{x})}{\partial x_{2}}\right)$ :

$$
\tilde{u}(\boldsymbol{x})=\underset{u(\boldsymbol{x}) \in \mathbb{R}}{\operatorname{argmin}}\{\underbrace{\frac{\beta_{1}}{2}\left|p(\boldsymbol{x})-\nabla u(\boldsymbol{x})+\frac{\lambda_{1}(\boldsymbol{x})}{\beta_{1}}\right|^{2}+\frac{\beta_{3}}{2}\left(f(\boldsymbol{x})-u(\boldsymbol{x})-v(\boldsymbol{x})-\epsilon(\boldsymbol{x})+\frac{\lambda_{3}(\boldsymbol{x})}{\beta_{3}}\right)^{2}}_{:=F(\boldsymbol{x}, u(\boldsymbol{x}), \nabla u(\boldsymbol{x}))}\},
$$


where the convex function $F: \Omega_{c} \times\left(\mathbb{R} \times \mathbb{R}^{2}\right) \rightarrow \mathbb{R} \cup\{ \pm \infty\}$ is addressed as

$$
\begin{aligned}
F(\boldsymbol{x}, u(\boldsymbol{x}), \nabla u(\boldsymbol{x})) & =\frac{\beta_{1}}{2}\left[\left(\partial_{x_{1}} u(\boldsymbol{x})-p_{1}(\boldsymbol{x})-\frac{\lambda_{1,1}(\boldsymbol{x})}{\beta_{1}}\right)^{2}+\left(\partial_{x_{2}} u(\boldsymbol{x})-p_{2}(\boldsymbol{x})-\frac{\lambda_{1,2}(\boldsymbol{x})}{\beta_{1}}\right)^{2}\right] \\
& +\frac{\beta_{3}}{2}\left(f(\boldsymbol{x})-u(\boldsymbol{x})-v(\boldsymbol{x})-\epsilon(\boldsymbol{x})+\frac{\lambda_{3}(\boldsymbol{x})}{\beta_{3}}\right)^{2} .
\end{aligned}
$$

In order to simplify the notation, we abbreviate $F(\boldsymbol{x}, u(\boldsymbol{x}), \nabla u(\boldsymbol{x}))$ as $F$. According to $[109$, p. 286], at the optimality condition, the Euler-Lagrange equation is expressed as

$$
-\frac{\partial F}{\partial u(\boldsymbol{x})}+\left[\frac{\partial}{\partial x_{1}} \cdot \frac{\partial F}{\partial\left(\partial_{x_{1}} u(\boldsymbol{x})\right)}+\frac{\partial}{\partial x_{2}} \cdot \frac{\partial F}{\partial\left(\partial_{x_{2}} u(\boldsymbol{x})\right)}\right]=0 .
$$

where:

$$
\begin{aligned}
\frac{\partial F}{\partial u(\boldsymbol{x})} & =-\beta_{3}\left(f(\boldsymbol{x})-u(\boldsymbol{x})-v(\boldsymbol{x})-\epsilon(\boldsymbol{x})+\frac{\lambda_{3}(\boldsymbol{x})}{\beta_{3}}\right) \\
\frac{\partial F}{\partial\left(\partial_{x_{1}} u(\boldsymbol{x})\right)} & =\beta_{1}\left(\partial_{x_{1}} u(\boldsymbol{x})-p_{1}(\boldsymbol{x})-\frac{\lambda_{1,1}(\boldsymbol{x})}{\beta_{1}}\right) \\
\Rightarrow \frac{\partial}{\partial x_{1}} \cdot \frac{\partial F}{\partial\left(\partial_{x_{1}} u(\boldsymbol{x})\right)} & =\beta_{1}\left(\partial_{x_{1}}^{2} u(\boldsymbol{x})-\partial_{x_{1}} p_{1}(\boldsymbol{x})-\frac{\partial_{x_{1}} \lambda_{1,1}(\boldsymbol{x})}{\beta_{1}}\right) \\
\frac{\partial F}{\partial\left(\partial_{x_{2}} u(\boldsymbol{x})\right)} & =\beta_{1}\left(\partial_{x_{2}} u(\boldsymbol{x})-p_{2}(\boldsymbol{x})-\frac{\lambda_{1,2}(\boldsymbol{x})}{\beta_{1}}\right) \\
\Rightarrow \frac{\partial}{\partial x_{2}} \cdot \frac{\partial F}{\partial\left(\partial_{x_{2}} u(\boldsymbol{x})\right)} & =\beta_{1}\left(\partial_{x_{2}}^{2} u(\boldsymbol{x})-\partial_{x_{2}} p_{2}(\boldsymbol{x})-\frac{\partial_{x_{2}} \lambda_{1,2}(\boldsymbol{x})}{\beta_{1}}\right)
\end{aligned}
$$

Therefore, the Euler Lagrange equation is

$$
\beta_{3} \tilde{u}(\boldsymbol{x})-\beta_{1} \Delta \tilde{u}(\boldsymbol{x})=\beta_{3}\left(f(\boldsymbol{x})-v(\boldsymbol{x})-\epsilon(\boldsymbol{x})+\frac{\lambda_{3}(\boldsymbol{x})}{\beta_{3}}\right)-\beta_{1} \operatorname{div}\left(p(\boldsymbol{x})+\frac{\lambda_{1}(\boldsymbol{x})}{\beta_{1}}\right) .
$$

The discrete version of the Euler Lagrange equation at $\boldsymbol{x}=\boldsymbol{k} \in \Omega_{d}$ is

$$
\beta_{3} \tilde{u}[\boldsymbol{k}]-\beta_{1} \Delta_{\mathrm{d}} \tilde{u}[\boldsymbol{k}]=\beta_{3}\left(f[\boldsymbol{k}]-v[\boldsymbol{k}]-\epsilon[\boldsymbol{k}]+\frac{\lambda_{3}[\boldsymbol{k}]}{\beta_{3}}\right)-\beta_{1} \operatorname{div}_{\mathrm{d}}\left(p[\boldsymbol{k}]+\frac{\lambda_{1}[\boldsymbol{k}]}{\beta_{1}}\right),
$$

where $\Delta_{\mathrm{d}}$ and $\operatorname{div}_{\mathrm{d}}$ are the discrete Laplacian and discrete divergence operators (cf. Appendix 3.7).

The vector form with the point-wise operators $(+,-, \times, /)$ is

$$
\beta_{3} \tilde{\boldsymbol{u}}-\beta_{1} \Delta_{\mathrm{d}} \tilde{\boldsymbol{u}}=\beta_{3}\left(\boldsymbol{f}-\boldsymbol{v}-\boldsymbol{\epsilon}+\frac{\boldsymbol{\lambda}_{\mathbf{3}}}{\beta_{3}}\right)-\beta_{1} \operatorname{div}_{\mathrm{d}}\left(\boldsymbol{p}+\frac{\boldsymbol{\lambda}_{\mathbf{1}}}{\beta_{1}}\right),
$$

where $\Delta_{\mathrm{d}} \boldsymbol{u}=\left[\Delta_{\mathrm{d}} u[\boldsymbol{k}]\right]_{\boldsymbol{k} \in \Omega_{d}}$ and $\operatorname{div}_{\mathrm{d}} \boldsymbol{u}=\left[\operatorname{div}_{\mathrm{d}} u[\boldsymbol{k}]\right]_{\boldsymbol{k} \in \Omega_{d}}$. 
By employing the discrete Fourier transform, the partial differential equation (3.30) is solved. Let $F\left(e^{j \boldsymbol{\omega}}\right), V\left(e^{j \boldsymbol{\omega}}\right), E\left(e^{j \boldsymbol{\omega}}\right), \Lambda_{3}\left(e^{j \boldsymbol{\omega}}\right), P_{1}\left(e^{j \boldsymbol{\omega}}\right), P_{2}\left(e^{j \boldsymbol{\omega}}\right)$ and $\Lambda_{1}\left(e^{j \boldsymbol{\omega}}\right)$ be the discrete Fourier transform of $f[\boldsymbol{k}], v[\boldsymbol{k}], \epsilon[\boldsymbol{k}], \lambda_{3}[\boldsymbol{k}], p_{1}[\boldsymbol{k}], p_{2}[\boldsymbol{k}]$ and $\lambda_{1}[\boldsymbol{k}]$, respectively. Due to the discreteness of the pixels, (3.30) is rewritten as

$$
\begin{array}{r}
\beta_{3} \tilde{u}[\boldsymbol{k}]-\beta_{1} \Delta_{\mathrm{d}} \tilde{u}[\boldsymbol{k}]=\beta_{3}\left(f[\boldsymbol{k}]-v[\boldsymbol{k}]-\epsilon[\boldsymbol{k}]+\frac{\lambda_{3}[\boldsymbol{k}]}{\beta_{3}}\right)-\beta_{1} \operatorname{div}_{\mathrm{d}}\left(p[\boldsymbol{k}]+\frac{\lambda_{1}[\boldsymbol{k}]}{\beta_{1}}\right) \\
\stackrel{\mathcal{F}}{\longleftrightarrow} \beta_{3} \tilde{U}\left(e^{j \boldsymbol{\omega}}\right)+\beta_{1} 4\left[\sin ^{2}\left(\frac{\omega_{1}}{2}\right)+\sin ^{2}\left(\frac{\omega_{2}}{2}\right)\right] \tilde{U}\left(e^{j \boldsymbol{\omega}}\right):=D\left(e^{j \boldsymbol{\omega}}\right), \quad \boldsymbol{\omega} \in \Omega_{\omega}, \quad
\end{array}
$$

where

$$
\begin{aligned}
D\left(e^{j \boldsymbol{\omega}}\right) & =\beta_{3}\left[F\left(e^{j \boldsymbol{\omega}}\right)-V\left(e^{j \boldsymbol{\omega}}\right)-E\left(e^{j \boldsymbol{\omega}}\right)+\frac{\Lambda_{3}\left(e^{j \boldsymbol{\omega}}\right)}{\beta_{3}}\right] \\
& -\beta_{1}\left[\left(1-e^{-j \omega_{1}}\right)\left(P_{1}\left(e^{j \boldsymbol{\omega}}\right)+\frac{\Lambda_{1,1}\left(e^{j \boldsymbol{\omega}}\right)}{\beta_{1}}\right)+\left(1-e^{-j \omega_{2}}\right)\left(P_{2}\left(e^{j \boldsymbol{\omega}}\right)+\frac{\Lambda_{1,2}\left(e^{j \boldsymbol{\omega}}\right)}{\beta_{1}}\right)\right] .
\end{aligned}
$$

Thus, the solution of the " $\boldsymbol{u}$-problem" is

$$
\tilde{u}[\boldsymbol{k}]=\operatorname{Re}\left[\mathcal{F}^{-1}\left\{\frac{D\left(e^{j \boldsymbol{\omega}}\right)}{\beta_{3}+4 \beta_{1}\left[\sin ^{2}\left(\frac{\omega_{1}}{2}\right)+\sin ^{2}\left(\frac{\omega_{2}}{2}\right)\right]}\right\}\right][\boldsymbol{k}], \quad \forall \boldsymbol{k} \in \Omega_{d} \text { and } \boldsymbol{\omega} \in \Omega_{\omega} .
$$

Although the solution of the " $\boldsymbol{u}$-problem" is obtained in the continuous setting and then is discretized as in the proof, it is not difficult to show that its solution can be directly solved in the discrete setting.

Denote $m$ and $n$ as the size of the domain $\Omega$ and given the matrix of difference operator as

$$
\mathbf{D}_{\mathbf{m}}=\left(\begin{array}{ccccc}
-1 & 1 & 0 & \ldots & 0 \\
0 & -1 & 1 & \ldots & 0 \\
\vdots & \vdots & \vdots & \ddots & \vdots \\
0 & 0 & 0 & \ldots & 1 \\
1 & 0 & 0 & \ldots & -1
\end{array}\right) \in \mathbb{R}^{m \times m}
$$

we have $\nabla_{d} \boldsymbol{u}=[\underbrace{\boldsymbol{u} \mathbf{D}_{\mathbf{n}}^{\mathrm{T}}}_{:=\partial_{x}^{+} \boldsymbol{u}}, \underbrace{\mathbf{D}_{\mathbf{m}} \boldsymbol{u}}_{:=\partial_{y}^{+} \boldsymbol{u}}]$ and $|\nabla u|=\sqrt{u_{x}^{2}+u_{y}^{2}}$,

$$
\tilde{\boldsymbol{u}}=\underset{\boldsymbol{u} \in X}{\operatorname{argmin}}\left\{F(\boldsymbol{u})=\frac{\beta_{1}}{2}\left\|\boldsymbol{p}-\nabla_{d} \boldsymbol{u}+\frac{\lambda_{\mathbf{1}}}{\beta_{1}}\right\|_{\ell_{2}}^{2}+\frac{\beta_{3}}{2}\left\|\boldsymbol{f}-\boldsymbol{u}-\boldsymbol{v}-\boldsymbol{\epsilon}+\frac{\lambda_{\mathbf{3}}}{\beta_{3}}\right\|_{\ell_{2}}^{2}\right\},
$$

where

$$
\left\|\boldsymbol{p}-\nabla_{d} \boldsymbol{u}+\frac{\boldsymbol{\lambda}_{\mathbf{1}}}{\beta_{1}}\right\|_{\ell_{2}}^{2}=\left\|\boldsymbol{p}_{2}-\boldsymbol{u} \mathbf{D}_{\mathbf{n}}^{\mathrm{T}}+\frac{\boldsymbol{\lambda}_{\mathbf{1} 2}}{\beta_{1}}\right\|_{\ell_{2}}^{2}+\left\|\boldsymbol{p}_{1}-\mathbf{D}_{\mathbf{m}} \boldsymbol{u}+\frac{\boldsymbol{\lambda}_{\mathbf{1 1}}}{\beta_{1}}\right\|_{\ell_{2}}^{2} .
$$

Thus,

$\tilde{\boldsymbol{u}}=\underset{\boldsymbol{u} \in X}{\operatorname{argmin}}\left\{F(\boldsymbol{u})=\frac{\beta_{1}}{2}\left\|\boldsymbol{p}_{2}-\boldsymbol{u} \mathbf{D}_{\mathbf{n}}^{\mathrm{T}}+\frac{\boldsymbol{\lambda}_{\mathbf{1} 2}}{\beta_{1}}\right\|_{\ell_{2}}^{2}+\frac{\beta_{1}}{2}\left\|\boldsymbol{p}_{1}-\mathbf{D}_{\mathbf{m}} \boldsymbol{u}+\frac{\boldsymbol{\lambda}_{\mathbf{1 1}}}{\beta_{1}}\right\|_{\ell_{2}}^{2}+\frac{\beta_{3}}{2}\left\|\boldsymbol{f}-\boldsymbol{u}-\boldsymbol{v}-\boldsymbol{\epsilon}+\frac{\boldsymbol{\lambda}_{\mathbf{3}}}{\beta_{3}}\right\|\right\}$ 
The Euler-Lagrange equation is

$$
\begin{gathered}
0=\frac{\partial F(\boldsymbol{u})}{\partial \boldsymbol{u}}=-\beta_{1}\left(\boldsymbol{p}_{2}-\boldsymbol{u} \mathbf{D}_{\mathbf{n}}^{\mathrm{T}}+\frac{\boldsymbol{\lambda}_{\mathbf{1} 2}}{\beta_{1}}\right) \mathbf{D}_{\mathbf{n}}-\beta_{1} \mathbf{D}_{\mathbf{m}}^{\mathrm{T}}\left(\boldsymbol{p}_{1}-\mathbf{D}_{\mathbf{m}} \boldsymbol{u}+\frac{\boldsymbol{\lambda}_{\mathbf{1 1}}}{\beta_{1}}\right)-\beta_{3}\left(\boldsymbol{f}-\boldsymbol{u}-\boldsymbol{v}-\boldsymbol{\epsilon}+\frac{\boldsymbol{\lambda}_{\mathbf{3}}}{\beta_{3}}\right) \\
\Leftrightarrow \beta_{1} \boldsymbol{u} \mathbf{D}_{\mathbf{n}}^{\mathrm{T}} \mathbf{D}_{\mathbf{n}}+\beta_{1} \mathbf{D}_{\mathbf{m}}^{\mathrm{T}} \mathbf{D}_{\mathbf{m}} \boldsymbol{u}+\beta_{3} \boldsymbol{u}=\beta_{1}\left(\boldsymbol{p}_{2}+\frac{\boldsymbol{\lambda}_{\mathbf{1}}}{\beta_{1}}\right) \mathbf{D}_{\mathbf{n}}+\beta_{1} \mathbf{D}_{\mathbf{m}}^{\mathrm{T}}\left(\boldsymbol{p}_{1}+\frac{\boldsymbol{\lambda}_{\mathbf{1 1}}}{\beta_{1}}\right)+\beta_{3}\left(\boldsymbol{f}-\boldsymbol{v}-\boldsymbol{\epsilon}+\frac{\boldsymbol{\lambda}_{\mathbf{3}}}{\beta_{3}}\right) .
\end{gathered}
$$

Thus, the Sylvester equation is

$\beta_{1} \boldsymbol{u} \mathbf{D}_{\mathbf{n}}^{\mathrm{T}} \mathbf{D}_{\mathbf{n}}+\left(\beta_{1} \mathbf{D}_{\mathbf{m}}^{\mathrm{T}} \mathbf{D}_{\mathbf{m}}+\beta_{3} \mathbf{1}_{\mathbf{m} \mathbf{n}}\right) \boldsymbol{u}=\beta_{1}\left(\boldsymbol{p}_{2}+\frac{\boldsymbol{\lambda}_{\mathbf{1 2}}}{\beta_{1}}\right) \mathbf{D}_{\mathbf{n}}+\beta_{1} \mathbf{D}_{\mathbf{m}}^{\mathrm{T}}\left(\boldsymbol{p}_{1}+\frac{\boldsymbol{\lambda}_{\mathbf{1 1}}}{\beta_{1}}\right)+\beta_{3}\left(\boldsymbol{f}-\boldsymbol{v}-\boldsymbol{\epsilon}+\frac{\boldsymbol{\lambda}_{\mathbf{3}}}{\beta_{3}}\right)$

The solution of Eq. (3.33) can be solved in the discrete Fourier domain as

$$
\begin{aligned}
& {\left[\beta_{1}\left[\left(e^{-j \omega_{1}}-1\right)\left(e^{j \omega_{1}}-1\right)+\left(e^{-j \omega_{2}}-1\right)\left(e^{j \omega_{2}}-1\right)\right]+\beta_{3}\right] U\left(e^{j \boldsymbol{\omega}}\right) } \\
= & \beta_{1}\left[\left(e^{-j \omega_{1}}-1\right)\left(P_{1}\left(e^{j \boldsymbol{\omega}}\right)+\frac{\Lambda_{11}\left(e^{j \boldsymbol{\omega}}\right)}{\beta_{1}}\right)+\left(e^{-j \omega_{2}}-1\right)\left(P_{2}\left(e^{j \boldsymbol{\omega}}\right)+\frac{\Lambda_{12}\left(e^{j \boldsymbol{\omega}}\right)}{\beta_{1}}\right)\right] \\
& +\beta_{3}\left[F\left(e^{j \boldsymbol{\omega}}\right)-V\left(e^{j \boldsymbol{\omega}}\right)-E\left(e^{j \boldsymbol{\omega}}\right)+\frac{\Lambda_{3}\left(e^{j \boldsymbol{\omega}}\right)}{\beta_{3}}\right] \\
= & D\left(e^{j \boldsymbol{\omega}}\right),
\end{aligned}
$$

where

$$
A\left(e^{j \boldsymbol{\omega}}\right)=\beta_{3}+\beta_{1}[\underbrace{\left(e^{-j \omega_{1}}-1\right)\left(e^{j \omega_{1}}-1\right)+\left(e^{-j \omega_{2}}-1\right)\left(e^{j \omega_{2}}-1\right)}_{=4\left[\sin ^{2} \frac{\omega_{1}}{2}+\sin ^{2} \frac{\omega_{2}}{2}\right]}]
$$

Thus, the solution of the "u"-problem in the Fourier domain is

$$
U\left(e^{j \omega}\right)=\frac{D\left(e^{j \omega}\right)}{\beta_{3}+4 \beta_{1}\left[\sin ^{2} \frac{\omega_{1}}{2}+\sin ^{2} \frac{\omega_{2}}{2}\right]} .
$$

\subsection{Discrete version of the gradient, the divergence and the Laplacian operators}

Based on [103], this section introduces the discrete version of the gradient, the divergence and the Laplacian operators, as well as their Fourier transform. 


\subsubsection{The forward and backward gradient operator}

Given $\boldsymbol{f} \in X$ and $\boldsymbol{k} \in \Omega_{d}$, the discrete gradient operator $\nabla_{\mathrm{d}} \boldsymbol{f}$ with periodic boundary condition is a vector in $Y$ given by

$$
\nabla_{\mathrm{d}} f[\boldsymbol{k}]:=\nabla^{ \pm} f[\boldsymbol{k}]=\left[\partial_{1}^{ \pm} f[\boldsymbol{k}], \partial_{2}^{ \pm} f[\boldsymbol{k}]\right]^{T},
$$

where the forward differential operators are

$$
\begin{aligned}
& \partial_{1}^{+} f\left[k_{1}, k_{2}\right]= \begin{cases}f\left[k_{1}+1, k_{2}\right]-f\left[k_{1}, k_{2}\right], & k_{1}<N_{1}-1 ; \\
f\left[0, k_{2}\right]-f\left[N_{1}-1, k_{2}\right], & k_{1}=N_{1}-1,\end{cases} \\
& \partial_{2}^{+} f\left[k_{1}, k_{2}\right]= \begin{cases}f\left[k_{1}, k_{2}+1\right]-f\left[k_{1}, k_{2}\right], & k_{2}<N_{2}-1 ; \\
f\left[k_{1}, 0\right]-f\left[k_{1}, N_{2}-1\right], & k_{2}=N_{2}-1 .\end{cases}
\end{aligned}
$$

and the backward differential operators are

$$
\begin{aligned}
& \partial_{1}^{-} f\left[k_{1}, k_{2}\right]= \begin{cases}f\left[k_{1}, k_{2}\right]-f\left[k_{1}-1, k_{2}\right], & k_{1} \leq N_{1}-1 ; \\
f\left[0, k_{2}\right]-f\left[N_{1}-1, k_{2}\right], & k_{1}=0,\end{cases} \\
& \partial_{2}^{-} f\left[k_{1}, k_{2}\right]= \begin{cases}f\left[k_{1}, k_{2}\right]-f\left[k_{1}, k_{2}-1\right], & k_{2} \leq N_{2}-1 ; \\
f\left[k_{1}, 0\right]-f\left[k_{1}, N_{2}-1\right], & k_{2}=0 .\end{cases}
\end{aligned}
$$

Their Fourier transforms are

$$
\partial_{m}^{ \pm} f[\boldsymbol{k}] \stackrel{\mathcal{F}}{\longleftrightarrow} \pm\left(e^{ \pm j \omega_{m}}-1\right) F\left(e^{j \boldsymbol{\omega}}\right), \quad \boldsymbol{\omega} \in \Omega_{\omega} \text { and } m \in\{1,2\} .
$$

By the $1^{\text {st }}$ order Maclaurin approximation, the approximated gradient operator is the continuous version:

$$
\begin{aligned}
\partial_{m}^{ \pm} f[\boldsymbol{k}] \stackrel{\mathcal{F}}{\longleftrightarrow} & \pm\left(e^{ \pm j \omega_{m}}-1\right) F\left(e^{j \boldsymbol{\omega}}\right) \\
& \left.\approx\left(j \omega_{m}\right) F\left(e^{j \boldsymbol{\omega}}\right) \stackrel{\mathcal{F}^{-1}}{\longleftrightarrow} \partial_{x_{m}} f(\boldsymbol{x})\right|_{\boldsymbol{x}=\boldsymbol{k} \in \Omega_{d}} .
\end{aligned}
$$

\subsubsection{The backward divergence operator}

Given $\boldsymbol{g}=\left[\boldsymbol{g}_{\mathbf{1}}, \boldsymbol{g}_{\mathbf{2}}\right]^{T} \in Y, \boldsymbol{k} \in \Omega_{d}$ and $\boldsymbol{\omega} \in \Omega_{\omega}$, the discrete backward divergence $\operatorname{div}_{\mathrm{d}}: Y \rightarrow X$ is

$$
\begin{aligned}
& \operatorname{div}_{\mathrm{d}}(g[\boldsymbol{k}]):=\operatorname{div}^{-}(g[\boldsymbol{k}])=\left\langle\nabla^{-}, g[\boldsymbol{k}]\right\rangle_{\ell_{2}}=\partial_{1}^{-} g_{1}[\boldsymbol{k}]+\partial_{2}^{-} g_{2}[\boldsymbol{k}] \\
& \stackrel{\mathcal{F}}{\longleftrightarrow}\left(1-e^{-j \omega_{1}}\right) G_{1}\left(e^{j \boldsymbol{\omega}}\right)+\left(1-e^{-j \omega_{2}}\right) G_{2}\left(e^{j \boldsymbol{\omega}}\right) .
\end{aligned}
$$

By the $1^{\text {st }}$ order Maclaurin approximation, the continuous divergence operator is

$$
\begin{aligned}
\operatorname{div}^{-}(g[\boldsymbol{k}]) \stackrel{\mathcal{F}}{\longleftrightarrow} & \left(1-e^{-j \omega_{1}}\right) G_{1}\left(e^{j \boldsymbol{\omega}}\right)+\left(1-e^{-j \omega_{2}}\right) G_{2}\left(e^{j \boldsymbol{\omega}}\right) . \\
& \approx\left(j \omega_{1}\right) G_{1}\left(e^{j \boldsymbol{\omega}}\right)+\left.\left(j \omega_{2}\right) G_{2}\left(e^{j \boldsymbol{\omega}}\right) \stackrel{\mathcal{F}^{-1}}{\longleftrightarrow} \operatorname{div}(g(\boldsymbol{x}))\right|_{\boldsymbol{x}=\boldsymbol{k} \in \Omega_{d}} .
\end{aligned}
$$




\subsubsection{The discrete Laplacian operator}

Given $\boldsymbol{f} \in X, \boldsymbol{k} \in \Omega_{d}$ and $\boldsymbol{\omega} \in \Omega_{\omega}$, the discrete Laplace transform $\Delta_{\mathrm{d}}(\cdot)=\operatorname{div}^{-} \nabla^{+}(\cdot)$ is

$$
\Delta_{\mathrm{d}} f[\boldsymbol{k}]=\operatorname{div}^{-} \nabla^{+} f[\boldsymbol{k}]=\left\langle\nabla^{-}, \nabla^{+} f[\boldsymbol{k}]\right\rangle_{\ell_{2}}=\left(\partial_{1}^{-} \partial_{1}^{+}+\partial_{2}^{-} \partial_{2}^{+}\right) f[\boldsymbol{k}] .
$$

The Fourier transform is

$$
\mathcal{F}\left\{\Delta_{\mathrm{d}} f[\boldsymbol{k}]\right\}=(\underbrace{\mathcal{F}\left\{\partial_{1}^{-}\right\} \cdot \mathcal{F}\left\{\partial_{1}^{+}\right\}+\mathcal{F}\left\{\partial_{2}^{-}\right\} \cdot \mathcal{F}\left\{\partial_{2}^{+}\right\}}_{L\left(e^{j \omega}\right)}) \cdot F\left(e^{j \omega}\right),
$$

where

$$
\begin{aligned}
L\left(e^{j \boldsymbol{\omega}}\right) & =-\left(e^{-j \omega_{1}}-1\right)\left(e^{j \omega_{1}}-1\right)-\left(e^{-j \omega_{2}}-1\right)\left(e^{j \omega_{2}}-1\right) \\
& =\left(e^{j \omega_{1}}+e^{-j \omega_{1}}\right)+\left(e^{j \omega_{2}}+e^{-j \omega_{2}}\right)-4 \\
& =2\left[\cos \left(\omega_{1}\right)+\cos \left(\omega_{2}\right)-2\right] \\
& =-4\left[\sin ^{2}\left(\frac{\omega_{1}}{2}\right)+\sin ^{2}\left(\frac{\omega_{2}}{2}\right)\right]:=-4\left\|\sin \left(\frac{\boldsymbol{\omega}}{2}\right)\right\|_{\ell_{2}}^{2} .
\end{aligned}
$$

By the first order Maclaurin expansion of the cosin function, the approximated Laplacian operator (or the continuous Laplacian operator) is

$$
L\left(e^{j \boldsymbol{\omega}}\right) \approx-\left(\omega_{1}^{2}+\omega_{2}^{2}\right)=-\|\boldsymbol{\omega}\|_{\ell_{2}}^{2}
$$

The generalized version of order $\frac{s}{2} \in \mathbb{R}_{+}$is

$$
\begin{aligned}
\Delta_{\mathrm{d}}^{\frac{s}{2}} f[\boldsymbol{k}] \stackrel{\mathcal{F}}{\longleftrightarrow} & (-1)^{\frac{s}{2}} 2^{s}\left\|\sin \left(\frac{\boldsymbol{\omega}}{2}\right)\right\|_{\ell_{2}}^{s} \cdot F\left(e^{j \boldsymbol{\omega}}\right) \\
& \left.\approx(-1)^{\frac{s}{2}}\|\boldsymbol{\omega}\|_{\ell_{2}}^{s} \cdot F\left(e^{j \boldsymbol{\omega}}\right) \stackrel{\mathcal{F}}{\longleftrightarrow} \Delta^{\frac{s}{2}} f(\boldsymbol{x})\right|_{\boldsymbol{x}=\boldsymbol{k} \in \Omega_{d}} .
\end{aligned}
$$


Given $\boldsymbol{f} \in X, \boldsymbol{g} \in Y, \boldsymbol{k} \in \Omega_{d}$ and $\boldsymbol{\omega} \in \Omega_{\omega}$ :

- The discrete gradient operator:

$$
\begin{aligned}
\partial_{m}^{ \pm} f[\boldsymbol{k}] \stackrel{\mathcal{F}}{\longleftrightarrow} & \pm\left(e^{ \pm j \omega_{m}}-1\right) F\left(e^{j \boldsymbol{\omega}}\right), \quad m \in\{1,2\}, \\
& \left.\approx\left(j \omega_{m}\right) F\left(e^{j \boldsymbol{\omega}}\right) \stackrel{\mathcal{F}^{-1}}{\longleftrightarrow} \partial_{x_{m}} f(\boldsymbol{x})\right|_{\boldsymbol{x}=\boldsymbol{k} \in \Omega_{d}} .
\end{aligned}
$$

- The discrete divergence operator:

$$
\begin{aligned}
& \operatorname{div}^{-}(g[\boldsymbol{k}])=\partial_{1}^{-} g_{1}[\boldsymbol{k}]+\partial_{2}^{-} g_{2}[\boldsymbol{k}] \\
& \stackrel{\mathcal{F}}{\longleftrightarrow}\left(1-e^{-j \omega_{1}}\right) G_{1}\left(e^{j \boldsymbol{\omega}}\right)+\left(1-e^{-j \omega_{2}}\right) G_{2}\left(e^{j \boldsymbol{\omega}}\right) . \\
& \approx\left(j \omega_{1}\right) G_{1}\left(e^{j \boldsymbol{\omega}}\right)+\left.\left(j \omega_{2}\right) G_{2}\left(e^{j \boldsymbol{\omega}}\right) \stackrel{\mathcal{F}^{-1}}{\longleftrightarrow} \operatorname{div}(g(\boldsymbol{x}))\right|_{\boldsymbol{x}=\boldsymbol{k} \in \Omega_{d}} .
\end{aligned}
$$

- The $s^{\text {th }}$ order discrete Laplacian operator $(s \in \mathbb{R})$ :

$$
\begin{aligned}
& \Delta_{\mathrm{d}}^{\frac{s}{2}} f[\boldsymbol{k}]=\left(\operatorname{div}^{-} \nabla^{+}\right)^{\frac{s}{2}} f[\boldsymbol{k}]=\left(\partial_{1}^{-} \partial_{1}^{+}+\partial_{2}^{-} \partial_{2}^{+}\right)^{\frac{s}{2}} f[\boldsymbol{k}] \\
& \stackrel{\mathcal{F}}{\longleftrightarrow}(-1)^{\frac{s}{2}} 2^{s}\left\|\sin \left(\frac{\boldsymbol{\omega}}{2}\right)\right\|_{\ell_{2}}^{s} \cdot F\left(e^{j \boldsymbol{\omega}}\right) \\
& \left.\approx(-1)^{\frac{s}{2}}\|\boldsymbol{\omega}\|_{\ell_{2}}^{s} \cdot F\left(e^{j \boldsymbol{\omega}}\right) \stackrel{\mathcal{F}^{-1}}{\longleftrightarrow} \Delta^{\frac{s}{2}} f(\boldsymbol{x})\right|_{\boldsymbol{x}=\boldsymbol{k} \in \Omega_{d}} .
\end{aligned}
$$

\subsection{Additional Figures}




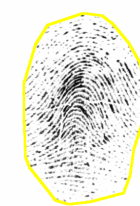

(a)

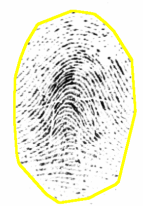

(b) $\operatorname{Err}=0.53$

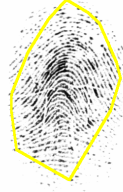

(e) $\operatorname{Err}=7.8$

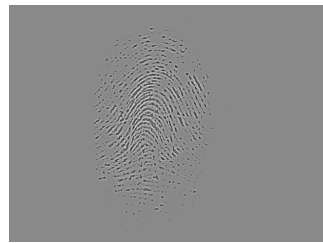

(h)

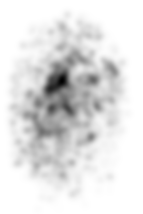

(1)

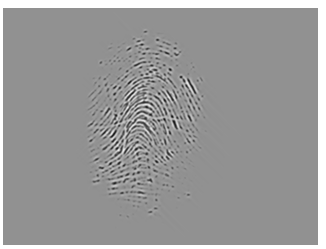

(i)

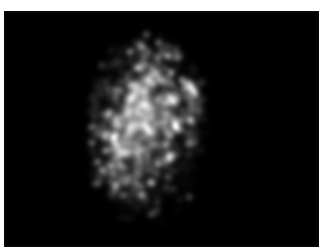

(m)

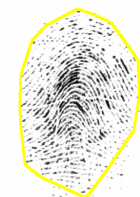

(c) $\operatorname{Err}=2.42$

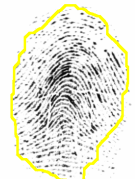

(f) $\operatorname{Err}=4.73$

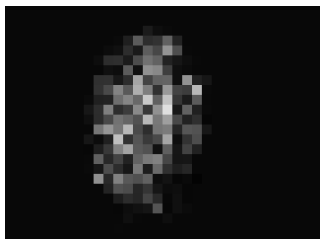

(j)

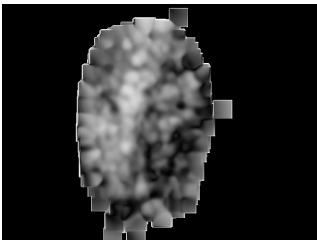

(n)

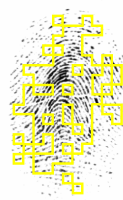

(d) $\operatorname{Err}=14.53$

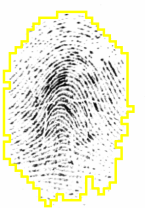

(g) $\operatorname{Err}=1.91$

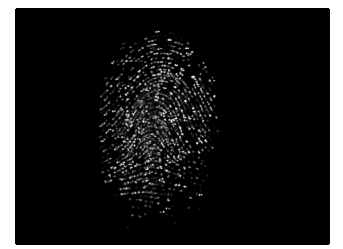

$(\mathrm{k})$

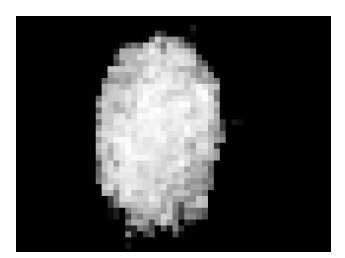

(o)

Figure 3.16: Segmented fingerprint images and their features of different methods for FVC2004_DB1_IM_4_7. (a) ground truth; (b, h) G3PD , (c, i) FDB, (d, j) Gabor, $(\mathrm{e}, \mathrm{k})$ Harris, (f, l, m, n) Mean-Variance-Coherence, (g, o) STFT. 


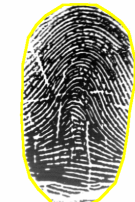

(a)

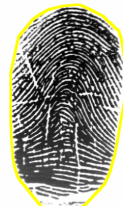

(b) $\operatorname{Err}=0.72$

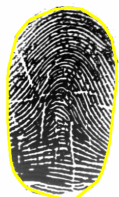

(e) $\operatorname{Err}=2.19$

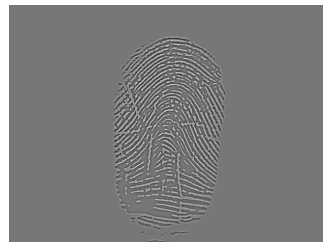

(h)

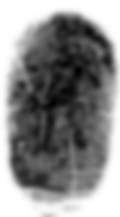

(1)

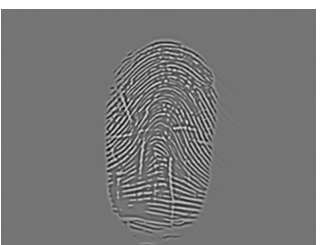

(i)

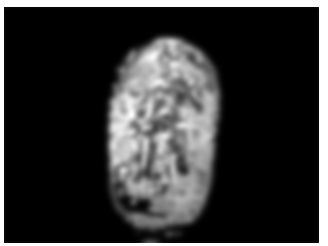

(m)

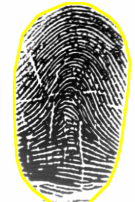

(c) $\operatorname{Err}=0.86$

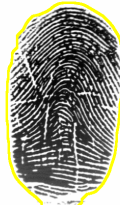

(f) $\operatorname{Err}=1.3$

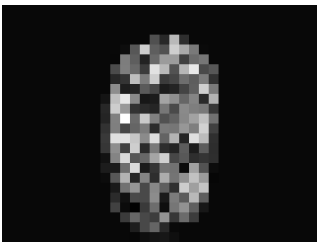

(j)

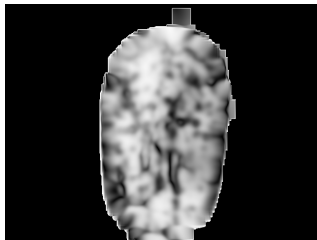

(n)

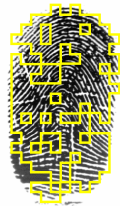

(d) $\operatorname{Err}=8.64$

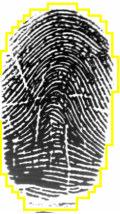

(g) $\operatorname{Err}=2.38$

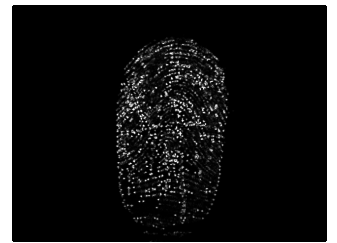

(k)

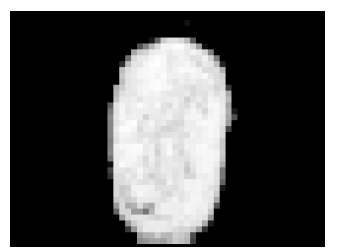

(o)

Figure 3.17: Segmented fingerprint images and their features of different methods for FVC2004_DB1_IM_4_8. (a) ground truth; (b, h) G3PD , (c, i) FDB, (d, j) Gabor, $(\mathrm{e}, \mathrm{k})$ Harris, (f, l, m, n) Mean-Variance-Coherence, (g, o) STFT. 


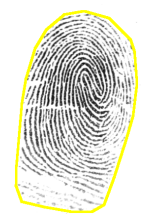

(a)

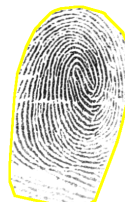

(b) $\operatorname{Err}=0.46$

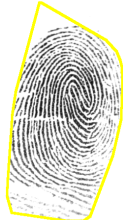

(e) $\operatorname{Err}=2.21$

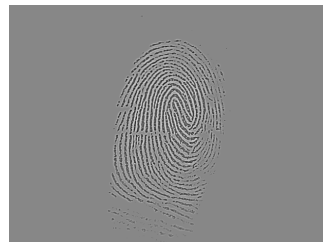

(h)

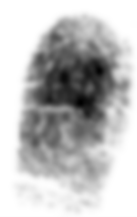

(1)

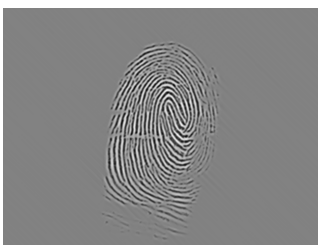

(i)

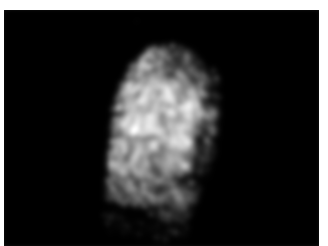

$(\mathrm{m})$

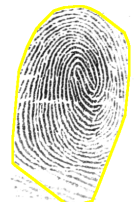

(c) $\operatorname{Err}=2.03$

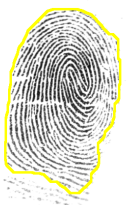

(f) $\operatorname{Err}=4.01$

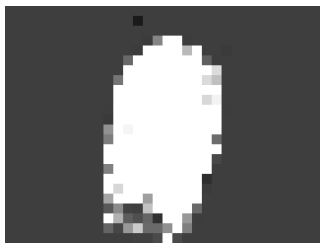

(j)

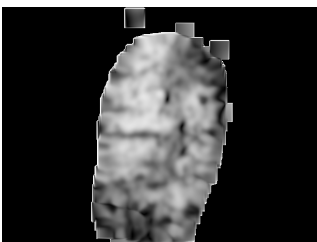

(n)

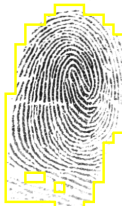

(d) $\operatorname{Err}=2.2$

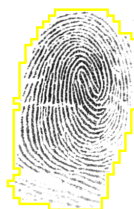

(g) $\operatorname{Err}=2.15$

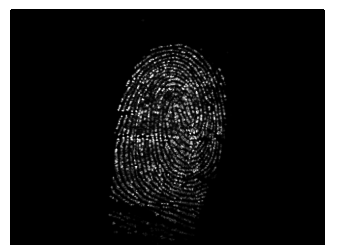

(k)

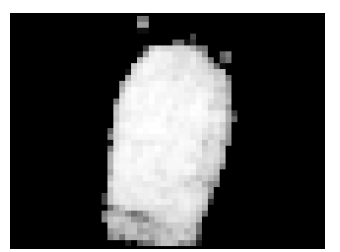

(o)

Figure 3.18: Segmented fingerprint images and their features of different methods for FVC2004_DB1_IM_49_5. (a) ground truth; (b, h) G3PD , (c, i) FDB, (d, j) Gabor, (e, k) Harris, (f, l, m, n) Mean-Variance-Coherence, (g, o) STFT. 


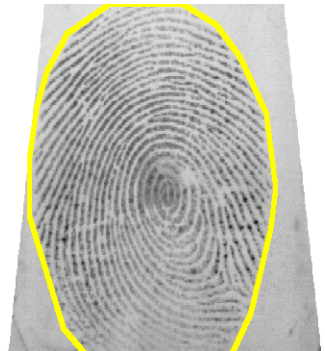

(a)

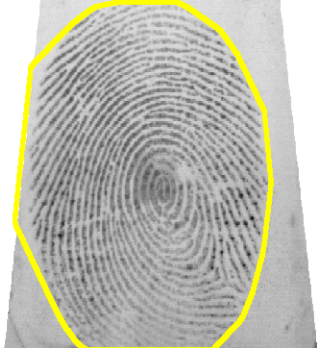

(b) $\operatorname{Err}=2.57$

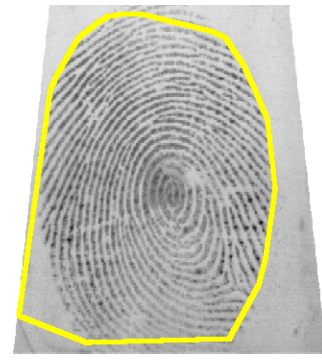

(e) $\operatorname{Err}=8.52$

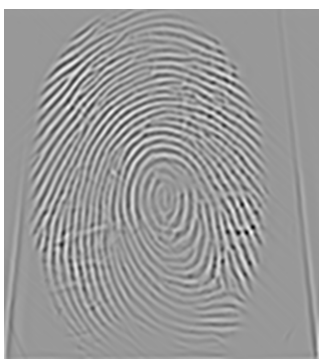

(i)

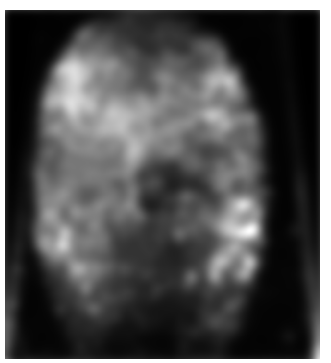

$(\mathrm{m})$

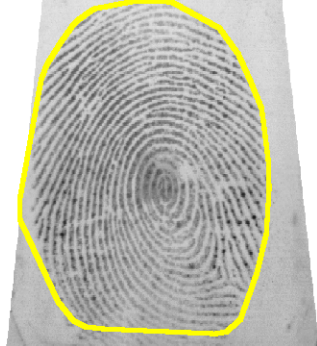

(c) $\operatorname{Err}=4.78$

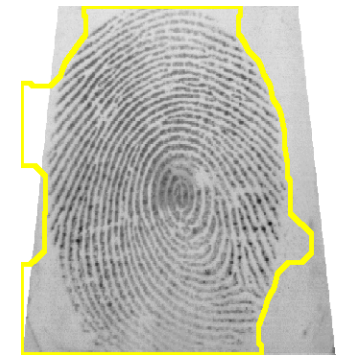

(f) $\operatorname{Err}=9.5$

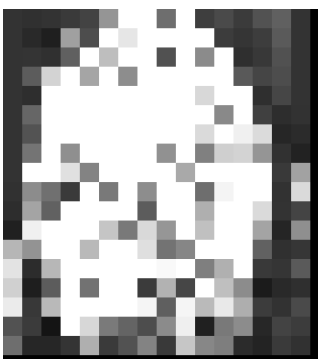

(j)

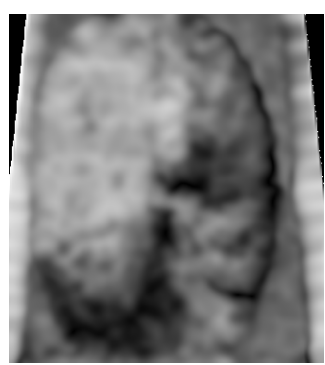

(n)

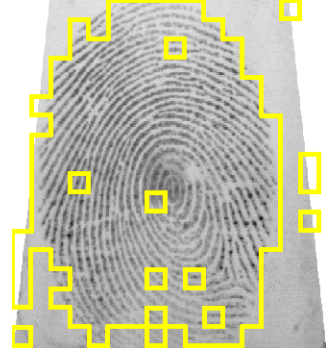

(d) $\operatorname{Err}=10.87$

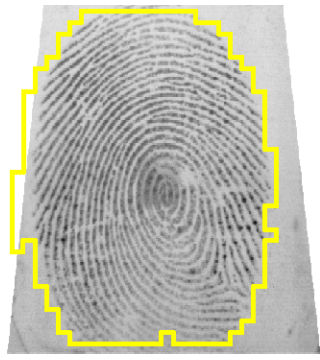

(g) $\operatorname{Err}=6$

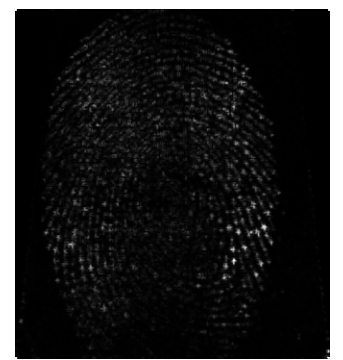

(k)

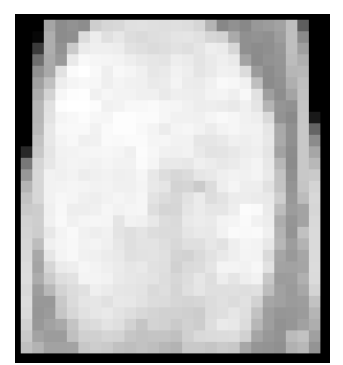

(o)

Figure 3.19: Segmented fingerprint images and their features of different methods for FVC2004_DB2_IM_43_6. (a) ground truth; (b, h) G3PD , (c, i) FDB, (d, j) Gabor, (e, k) Harris, (f, l, m, n) Mean-Variance-Coherence, (g, o) STFT. 


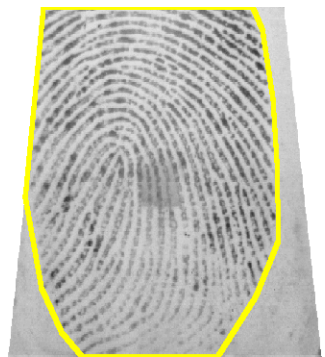

(a)

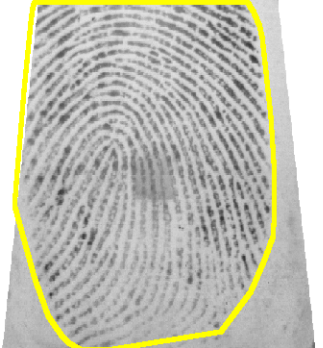

(b) $\operatorname{Err}=3.01$

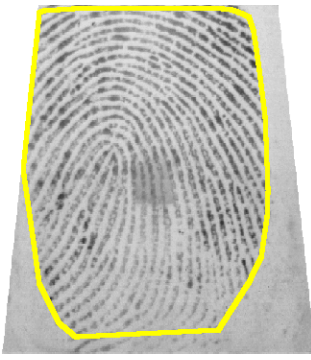

(e) $\operatorname{Err}=6$

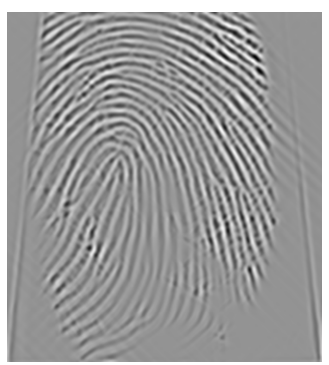

(i)

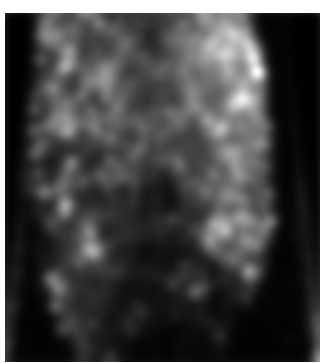

$(\mathrm{m})$

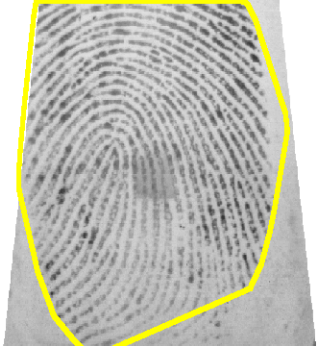

(c) $\operatorname{Err}=5.6$

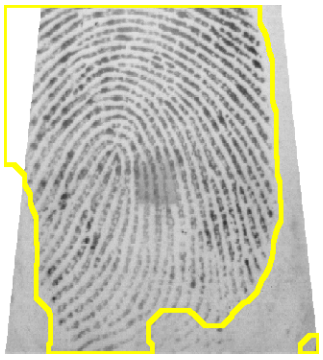

(f) $\operatorname{Err}=9.33$

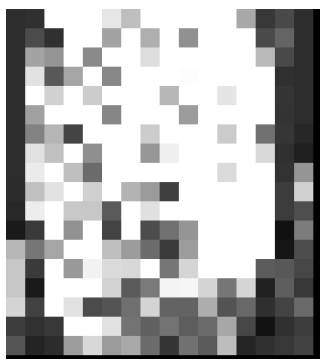

(j)

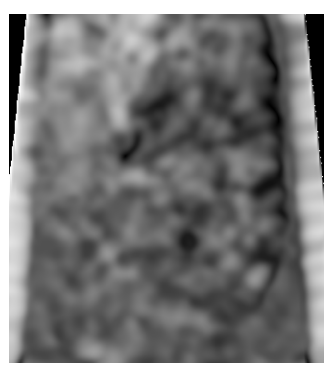

(n)

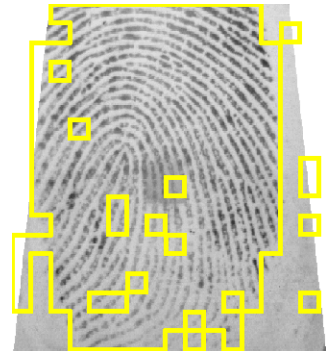

(d) $\operatorname{Err}=11.78$

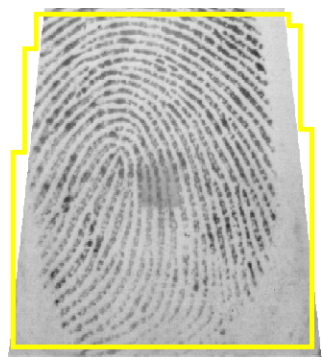

(g) $\operatorname{Err}=20.4$

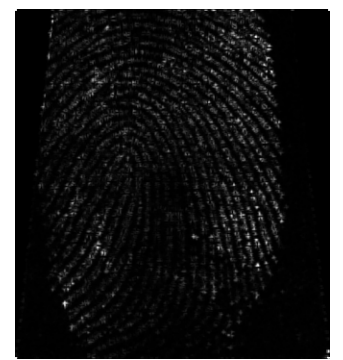

(k)

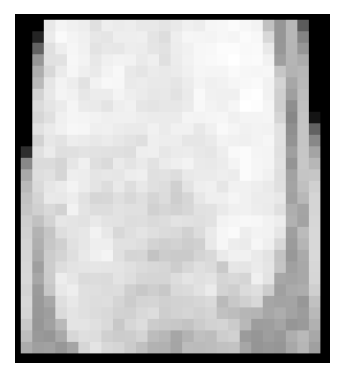

(o)

Figure 3.20: Segmented fingerprint images and their features of different methods for FVC2004_DB2_IM_47_6. (a) ground truth; (b, h) G3PD , (c, i) FDB, (d, j) Gabor, (e, k) Harris, (f, l, m, n) Mean-Variance-Coherence, (g, o) STFT. 


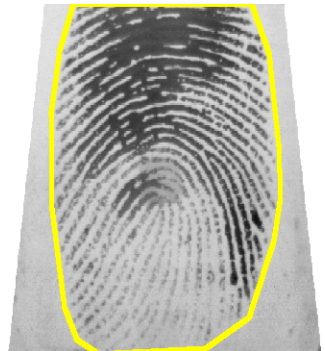

(a)

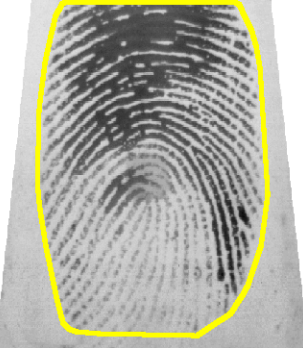

(b) $\operatorname{Err}=2.83$

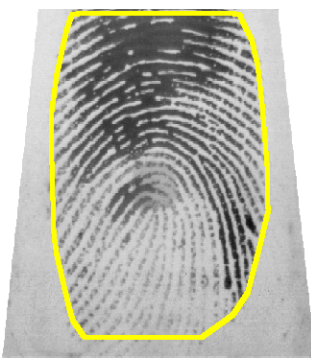

(e) $\operatorname{Err}=7.06$

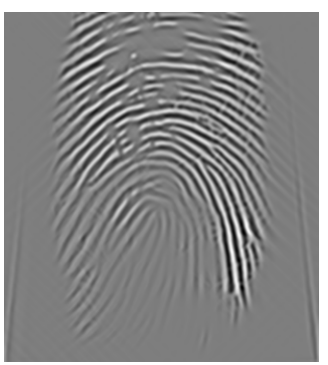

(i)

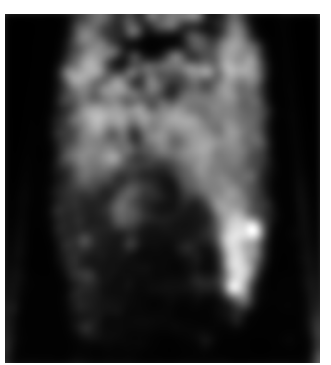

$(\mathrm{m})$

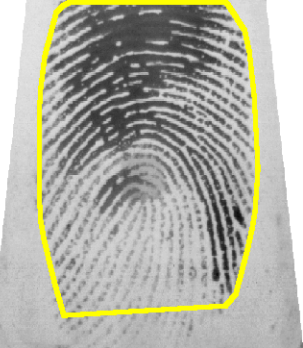

(c) $\operatorname{Err}=8.16$

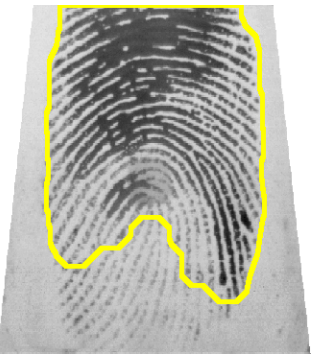

(f) $\operatorname{Err}=16.65$

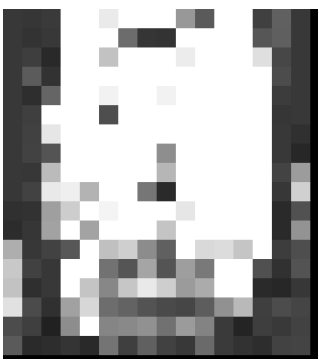

(j)

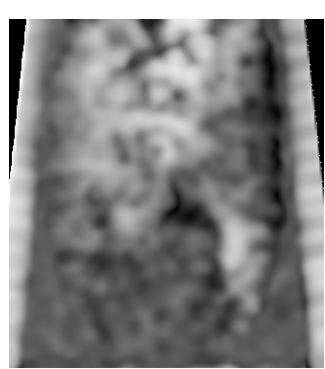

(n)

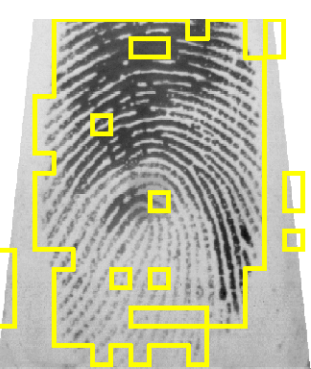

(d) $\operatorname{Err}=12.05$

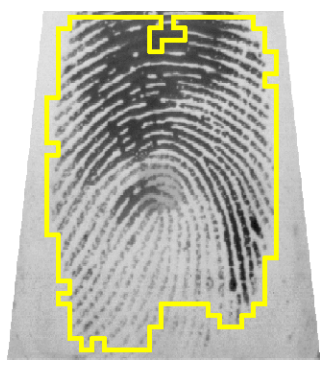

(g) $\operatorname{Err}=7.31$

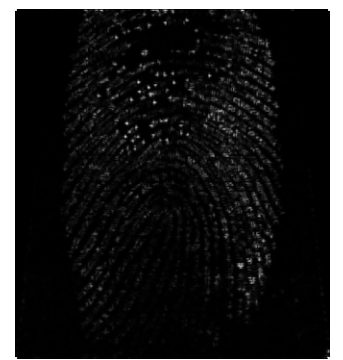

(k)

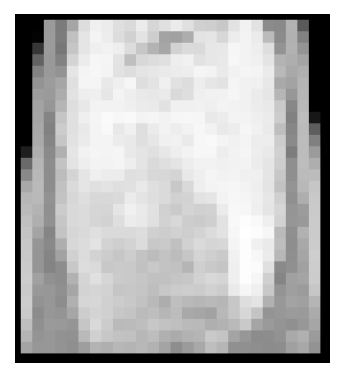

(o)

Figure 3.21: Segmented fingerprint images and their features of different methods for FVC2004_DB2_IM_47_7. (a) ground truth; (b, h) G3PD , (c, i) FDB, (d, j) Gabor, (e, k) Harris, (f, l, m, n) Mean-Variance-Coherence, (g, o) STFT. 


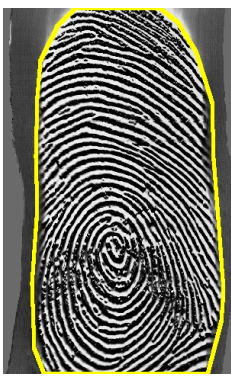

(a)

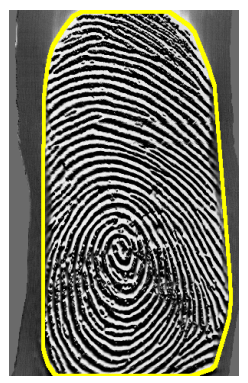

(b) $\operatorname{Err}=1.51$

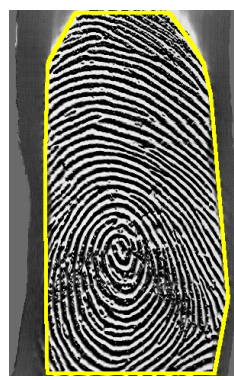

(e) $\operatorname{Err}=3.75$

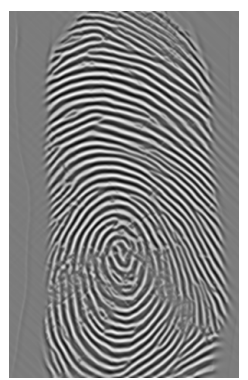

(i)

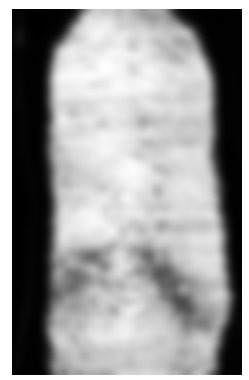

(m)

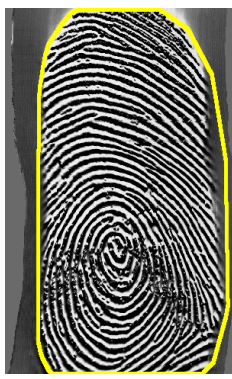

(c) $\operatorname{Err}=2.73$

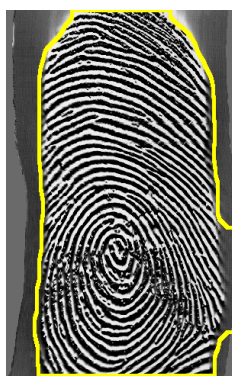

(f) $\operatorname{Err}=2.71$

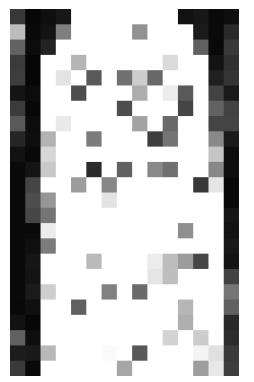

$(\mathrm{j})$

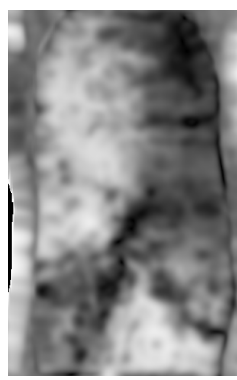

(n)

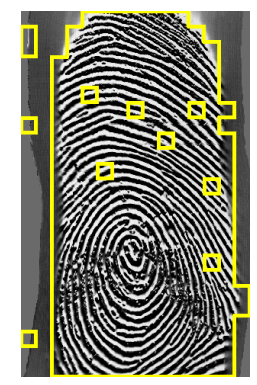

(d) $\operatorname{Err}=6.21$

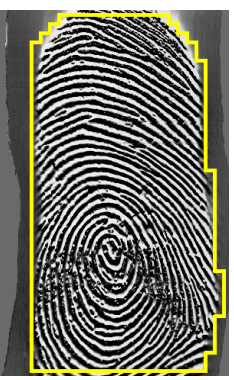

(g) Err $=3.92$

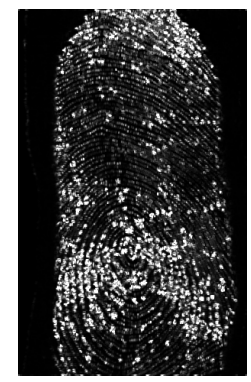

(k)

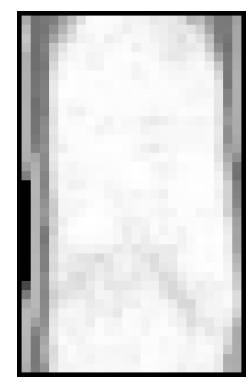

(o)

Figure 3.22: Segmented fingerprint images and their features of different methods for FVC2004_DB3_IM_1_7. (a) ground truth; (b, h) G3PD , (c, i) FDB, (d, j) Gabor, (e, k) Harris, (f, l, m, n) Mean-Variance-Coherence, (g, o) STFT. 


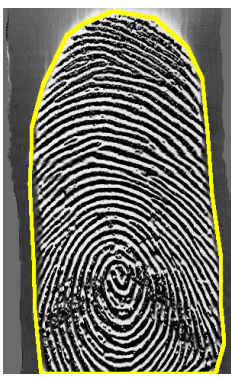

(a)

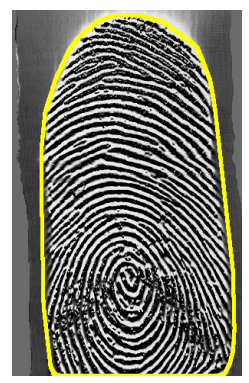

(b) $\operatorname{Err}=1.09$

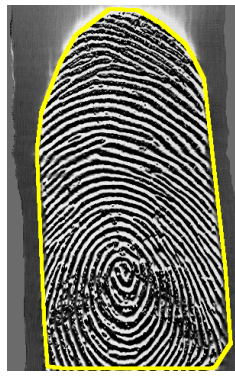

(e) $\operatorname{Err}=3.23$

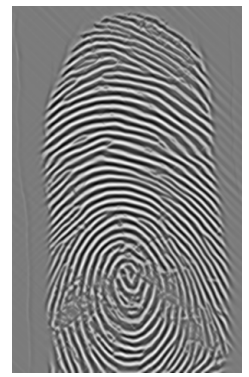

(i)

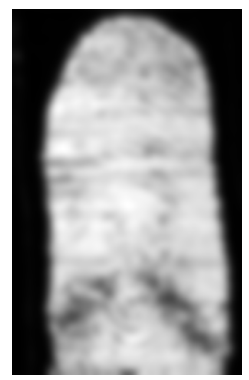

(m)

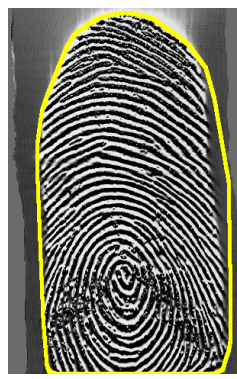

(c) $\operatorname{Err}=3.33$

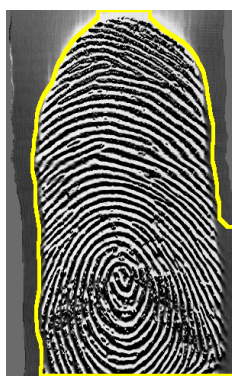

(f) $\operatorname{Err}=3.7$

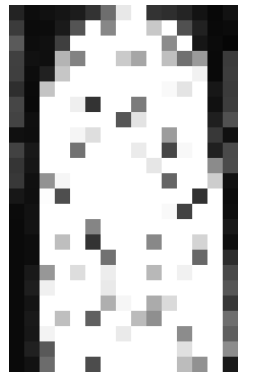

$(\mathrm{j})$

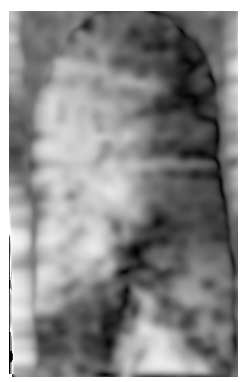

(n)

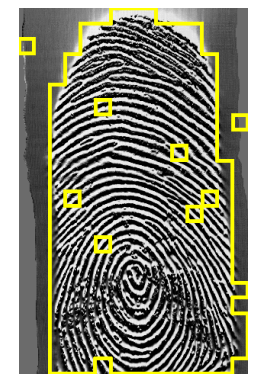

(d) $\operatorname{Err}=6.6$

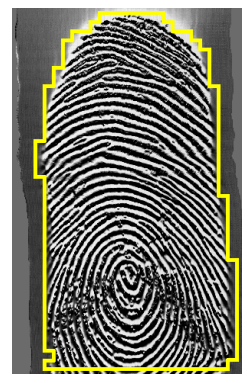

(g) $\operatorname{Err}=4.03$

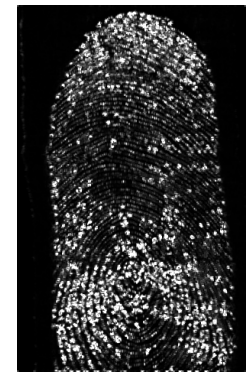

(k)

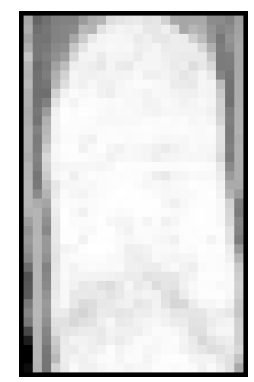

(o)

Figure 3.23: Segmented fingerprint images and their features of different methods for FVC2004_DB3_IM_1_8. (a) ground truth; (b, h) G3PD , (c, i) FDB, (d, j) Gabor, (e, k) Harris, (f, l, m, n) Mean-Variance-Coherence, (g, o) STFT. 


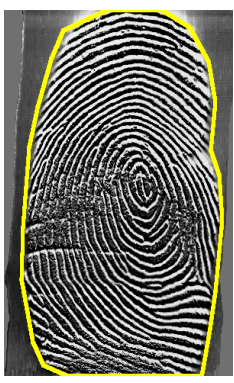

(a)

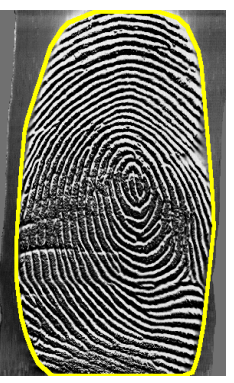

(b) $\operatorname{Err}=1.75$

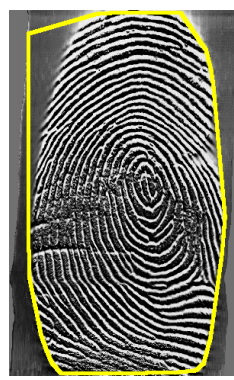

(e) $\operatorname{Err}=5.99$

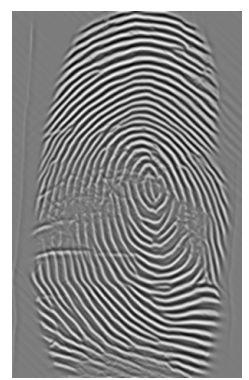

(i)

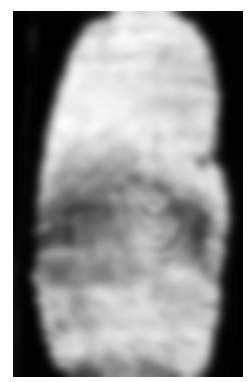

(m)

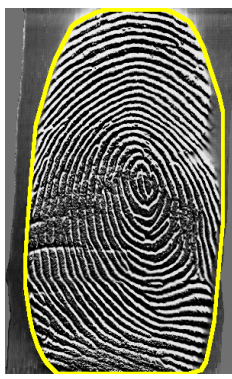

(c) $\operatorname{Err}=2.37$

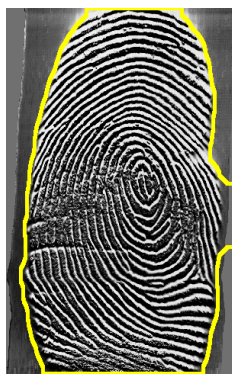

(f) $\operatorname{Err}=3.06$

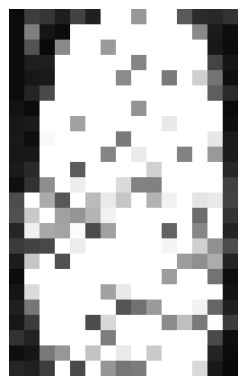

(j)

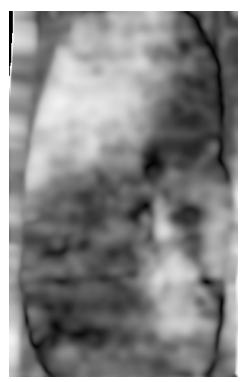

(n)

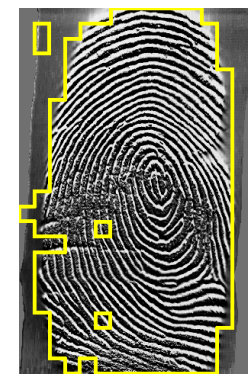

(d) $\operatorname{Err}=6$

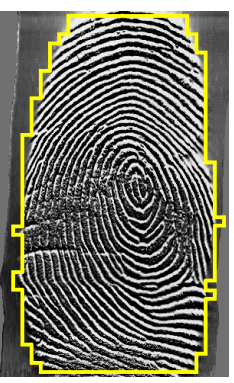

(g) $\operatorname{Err}=3.74$

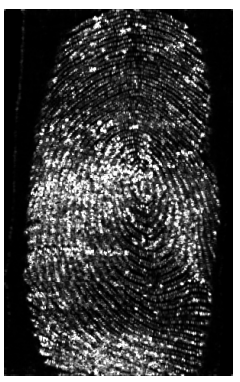

(k)

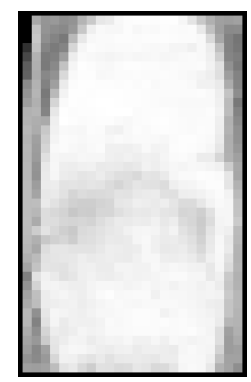

(o)

Figure 3.24: Segmented fingerprint images and their features of different methods for FVC2004_DB3_IM_19_4. (a) ground truth; (b, h) G3PD , (c, i) FDB, (d, j) Gabor, (e, k) Harris, (f, l, m, n) Mean-Variance-Coherence, (g, o) STFT. 


\section{Conclusion}

We have proposed the two models for fingerprint feature extraction, including: the Fourier based and variational based methods. We have demonstrated their potential application to fingerprint segmentation. The main contributions of this thesis are summarized in the following section and we also discuss the future work to conclude the study.

\subsection{Main Contributions}

There are threefolds of our contribution in this thesis:

- Firstly, we consider the methods for fingerprint feature extraction in the Fourier and the spatial domains. Based on the properties of fingerprint patterns, e.g. sparsity, smoothness and FOTIs, we design the FDB method [1] and the G3PD method [2] to compromise these properties. In particular, we design the FDB segmentation method for the feature extraction based on the DHBB filter interwoven with soft thresholding. In the different view with the FDB, the G3PD method is proposed to consider fingerprint pattern as a texture for the decomposition under the convex minimization, $\mathrm{TV}-G_{1,1}^{3 / 4}-\ell_{1}-G_{\infty, \infty}^{-3 / 4}$. After feature extraction by both approaches, the morphological operator is applied on these textures to obtain the ROI.

- Secondly, in order to clarify the performance of our proposed methods, we provide a manually marked ground truth segmentation for all 12 databases of FVC2000 [4], FVC2002 [5] and FVC2004 [6]. Each databases consists of 80 images for training and 800 images for testing. Overall this benchmark consists of 10560 marked segmentation images.

- Finally, we perform a systematic comparison of our proposed approaches with the four well-known fingerprint segmentation methods on this benchmark: a method based on mean and variance of gray level intensities and the coherence of gradients as features and a neural network as a classifier [7], a method using Gabor filter bank responses [8], a Harris corner response based method [9] and an approach using local Fourier analysis [10].

From the performance result in Table 3.3, we see that our proposed approaches give better results than the others in the literature. Especially, the performance of the variational based method for fingerprint segmentation gives the best result in general. The reason is that the convex minimization function simultaneously takes into account the balance between the sparsity and the smoothness of texture staying in different spaces, e.g. $\ell_{1}$ space and $G_{1,1}^{3 / 4}$ space, together with noise and homogeneous region in different kinds of norm. 


\section{Data accessibility:}

- Benchmark for Fingerprint Segmentation Performance Evaluation: http://dx.doi.org/10.6084/m9.figshare.1294209

- Matlab Implementation of the FDB Method for Fingerprint Segmentation http://dx.doi.org/10.6084/m9.figshare.1294210

- Matlab Implementation of the G3PD Method for Fingerprint Segmentation http://dx.doi.org/10.6084/m9.figshare.1418020

- FVC databases

http://bias.csr.unibo.it/fvc2000/

http://bias.csr.unibo.it/fvc2002/

http://bias.csr.unibo.it/fvc2004/

\subsection{Future Work}

Based on the properties of fingerprint pattern, we have considered the problem from the theoretical point of views to the practical application for fingerprint segmentation. However, there are many challenging issues in practice for further investigations:

- dryness or wetness of the finger

- a ghost fingerprint on the sensor surface

- small scale noise

- large scale structure noise

- image artifacts e.g. caused by reconstructing a swipe sensor image

- scars or creases interrupting the fingerprint pattern

- latent fingerprint image segmentation

- overlapped fingerprint segmentation

A relevant future direction is to build the mathematical model to overcome these difficulties in practice under different views, e.g. statistical methods, stochastic processes, partial differential equation, non-convex minimization and multiscale approach, etc. 


\section{Bibliography}

[1] D.H. Thai, S. Huckemann, and C. Gottschlich. Filter design and performance evaluation for fingerprint image segmentation. arXiv:1501.02113 [cs.CV], January 2015.

[2] D.H. Thai and C. Gottschlich. Global variational method for fingerprint segmentation by three-part decomposition. arXiv:1505.04585 [cs.CV], May 2015.

[3] D. Maltoni, D. Maio, A. K. Jain, and S. Prabhakar. Handbook of Fingerprint Recognition. Springer, London, U.K., 2009.

[4] D. Maio, D. Maltoni, R. Capelli, J. L. Wayman, and A. K. Jain. FVC2000: Fingerprint verification competition. IEEE Transactions on Pattern Analysis and Machine Intelligence, 24(3):402-412, March 2002.

[5] D. Maio, D. Maltoni, R. Capelli, J. L. Wayman, and A. K. Jain. FVC2002: Second fingerprint verification competition. In Proc. ICPR, pages 811-814, 2002.

[6] D. Maio, D. Maltoni, R. Capelli, J. L. Wayman, and A. K. Jain. FVC2004: Third fingerprint verification competition. In Proc. ICBA, pages 1-7, Hong Kong, 2004.

[7] A.M. Bazen and S.H. Gerez. Segmentation of fingerprint images. In Proc. ProRISC, pages 276-280, Veldhoven, The Netherlands, November 2001.

[8] L.L. Shen, A. Kot, and W.M. Koo. Quality measures of fingerprint images. In Proc. AVBPA, pages 266-271, Halmstad, Schweden, June 2001.

[9] C. Wu, S. Tulyakov, and V. Govindaraju. Robust point-based feature fingerprint segmentation algorithm. In Proc. ICB 200\%, pages 1095-1103, Seoul, Korea, August 2007.

[10] S. Chikkerur, A. Cartwright, and V. Govindaraju. Fingerprint image enhancement using STFT analysis. Pattern Recognition, 40(1):198-211, 2007.

[11] C. Gottschlich. Curved-region-based ridge frequency estimation and curved Gabor filters for fingerprint image enhancement. IEEE Transactions on Image Processing, 21(4):2220-2227, April 2012.

[12] C. Gottschlich, T. Hotz, R. Lorenz, S. Bernhardt, M. Hantschel, and A. Munk. Modeling the growth of fingerprints improves matching for adolescents. IEEE Transactions on Information Forensics and Security, 6(3):1165-1169, September 2011.

[13] B.M. Mehtre, N.N. Murthy, S. Kapoor, and B. Chatterjee. Segmentation of fingerprint images using the directional image. Pattern Recognition, 20(4):429435, August 1987.

[14] B.M. Mehtre and B. Chatterjee. Segmentation of fingerprint images - a composite 
method. Pattern Recognition, 22(4):381-385, August 1989.

[15] X. Chen, J. Tian, J. Cheng, and X. Yang. Segmentation of fingerprint images using linear classifier. EURASIP Journal on Applied Signal Processing, 2004(4):480-494, 2004.

[16] L. Wang, H. Suo, and M. Dai. Fingerprint image segmentation based on gaussianhermite moments. In Proc. ADMA, pages 446-454, Wuhan, China, July 2005.

[17] E. Zhu, J. Yin, C. Hu, and G. Zhang. A systematic method for fingerprint ridge orientation estimation and image segmentation. Pattern Recognition, 39(8):14521472, August 2006.

[18] B.G. Sherlock, D.M. Monro, and K. Millard. Fingerprint enhancement by directional Fourier filtering. IEE Proc. Vision, Image and Signal Processing, 141(2):87-94, April 1994.

[19] P. Sutthiwichaiporn and V. Areekul. Adaptive boosted spectral filtering for progressive fingerprint enhancement. Pattern Recognition, 46(9):2465-2486, September 2013.

[20] J.S. Bartůněk, M. Nilsson, J. Nordberg, and I. Claesson. Adaptive fingerprint binarization by frequency domain analysis. In Proc. ACSSC, pages 598-602, Pacific Grove, CA, USA, October 2006.

[21] J.S. Bartůněk, M. Nilsson, J. Nordberg, and I. Claesson. Improved adaptive fingerprint binarization. In Proc. CISP, pages 756-760, Sanya, China, May 2008.

[22] J.S. Bartůněk, M. Nilsson, B. Sällberg, and I. Claesson. Adaptive fingerprint image enhancement with emphasis on preprocessing of data. IEEE Transactions on Image Processing, 22(2):644-656, February 2013.

[23] J. Zhang, R. Lai, and C.-C.J. Kuo. Adaptive directional total-variation model for latent fingerprint segmentation. IEEE Transactions on Information Forensics and Security, 8(8):1261-1273, August 2013.

[24] K. Cao, E. Liu, and A.K. Jain. Segmentation and enhancement of latent fingerprints: A coarse to fine ridge structure dictionary. IEEE Transactions on Pattern Analysis and Machine Intelligence, 36(9):1847-1859, September 2014.

[25] M. Unser and D. Van De Ville. Wavelet steerability and the higher-order Riesz transform. IEEE Transactions on Image Processing, 19(3):636-652, March 2010.

[26] S. Held, M. Storath, P. Massopust, and B. Forster. Steerable wavelet frames based on the Riesz transform. IEEE Transactions on Image Processing, 19(3):653-667, March 2010.

[27] M. Unser, N. Chenouard, and D. Van De Ville. Steerable pyramids and tight wavelet frames in $L_{2}\left(\mathbb{R}^{d}\right)$. IEEE Transactions on Image Processing, 20(10):27052721, October 2011.

[28] J. Ma and G. Plonka. The curvelet transform. IEEE Signal Processing Magazin, 27(2):118-133, March 2010.

[29] E. Candès, L. Demanet, D. Donoho, and L. Ying. Fast discrete curvelet transforms. Multiscale Model. Simul., 5(3):861-899, September 2006. 
[30] M. Unser. Sampling - 50 years after Shannon. Proceedings of the IEEE, 88(4):569587, April 2000.

[31] M. Haltmeier and A. Munk. Extreme value analysis of empirical frame coefficients and implications for denoising by soft-thresholding. Applied and Computational Harmonic Analysis, 36(3):434-460, May 2014.

[32] J.G. Proakis and D.K. Manolakis. Digital Signal Processing: Principles, Algorithms, and Applications. Prentice Hall, Upper Saddle River, NJ, USA, 2007.

[33] J.H. McClellan. The design of two-dimensional digital filters by transformations. In Proc. APCIS, pages 247-251, Princeton, NJ, USA, 1973.

[34] R.M. Mersereau, W.F.G. Mecklenbräuker, and Jr. T.F. Quatieri. McClellan transformations for two-dimensional digital filtering: I - design. IEEE Transactions on Circuits and Systems, 23(7):405-414, July 1976.

[35] W.F.G. Mecklenbräuker and R.M. Mersereau. McClellan transformations for twodimensional digital filtering: II - implementation. IEEE Transactions on Circuits and Systems, 23(7):414-422, July 1976.

[36] C.-C. Tseng. Design of two-dimensional FIR digital filters by McClellan transform and quadratic programming. IEE Proceedings - Vision, Image and Signal Processing, 148(5):325-331, October 2001.

[37] M. Kočevar, B. Kotnik, A. Chowdhury, and Z. Kačič. Real-time fingerprint image enhancement with a two-stage algorithm and block-local normalization. Journal of Real-Time Image Processing, pages 1-10, July 2014.

[38] M. Ghafoor, I.A. Taj, W. Ahmad, and N.M. Jafri. Efficient 2-fold contextual filtering approach for fingerprint enhancement. IET Image Processing, 8(7):417425, July 2014.

[39] J. Yang, N. Xiong, and A.V. Vasilakos. Two-stage enhancement scheme for lowquality fingerprint images by learning from the images. IEEE Transactions on Human-Machine Systems, 43(2):235-248, March 2013.

[40] S. Yi, D. Labate, G.R. Easley, and H. Krim. A shearlet approach to edge analysis and detection. IEEE Transactions on Image Processing, 18(5):929-941, May 2009 .

[41] M.N. Do and M. Vetterli. The contourlet transform: An efficient directional multiresolution image representation. IEEE Transactions on Image Processing, 14(12):2091-2106, December 2005.

[42] K.N. Chaudhury and M. Unser. Construction of Hilbert transform pairs of wavelet bases and Gabor-like transforms. IEEE Transactions on Signal Processing, 57(9):3411-3425, September 2009.

[43] M. Felsberg and G. Sommer. The monogenic signal. IEEE Transactions on Signal Processing, 49(12):3136-3144, December 2001.

[44] S.L. Hahn. Hilbert transforms in signal processing. Artech House, Boston, MA, USA, 1996.

[45] K. G. Larkin, D. J. Bone, and M. A. Oldfield. Natural demodulation of two- 
dimensional fringe patterns. I. General background of the spiral phase quadrature transform. J. Opt. Soc. Am. A, 18(8):1862-1870, August 2001.

[46] K. G. Larkin. Natural demodulation of two-dimensional fringe patterns. II. Stationary phase analysis of the spiral phase quadrature transform. J. Opt. Soc. Am. A, 18(8):1871-1881, August 2001.

[47] K. G. Larkin and P. A. Fletcher. A coherent framework for fingerprint analysis: Are fingerprints holograms? Optics Express, 15(14):8667-8677, 2007.

[48] M. Unser, D. Sage, and D. Van De Ville. Multiresolution monogenic signal analysis using the Riesz-Laplace wavelet transform. IEEE Transactions on Image Processing, 18(11):2402-2418, November 2009.

[49] S. Häuser, B. Heise, and G. Steidl. Linearized Riesz transform and quasimonogenic shearlets. International Journal of Wavelets, Multiresolution and Information Processing, 12(3), May 2014.

[50] D.L. Donoho and J.M. Johnstone. Ideal spatial adaptation by wavelet shrinkage. Biometrika, 81(3):425-455, August 1994.

[51] T.F. Sanam and C. Shahnaz. A semisoft thresholding method based on Teager energy operation on wavelet packet coefficients for enhancing noisy speech. EURASIP Journal on Audio, Speech, and Music Processing, 2013(1):1-15, November 2013.

[52] M. Nasri and H. Nezamabadi-pour. Image denoising in the wavelet domain using a new adaptive thresholding function. Neurocomputing, 72(4-6):1012-1025, January 2009 .

[53] M. Sonka, V. Hlavac, and R. Boyle. Image Processing, Analysis, and Machine Vision. Thomson, Toronto, Canada, 2008.

[54] C. Gottschlich and S. Huckemann. Separating the real from the synthetic: Minutiae histograms as fingerprints of fingerprints. IET Biometrics, to appear, DOI: 10.1049/iet-bmt.2013.0065 .

[55] C. Gottschlich and D. Schuhmacher. The shortlist method for fast computation of the earth mover's distance and finding optimal solutions to transportation problems. PLOS ONE, 9(10):e110214, October 2014.

[56] C. Gottschlich, P. Mihăilescu, and A. Munk. Robust orientation field estimation and extrapolation using semilocal line sensors. IEEE Transactions on Information Forensics and Security, 4(4):802-811, December 2009.

[57] S. Huckemann, T. Hotz, and A. Munk. Global models for the orientation field of fingerprints: an approach based on quadratic differentials. IEEE Transactions on Pattern Analysis and Machine Intelligence, 30(9):1507-1517, September 2008.

[58] C. Gottschlich, E. Marasco, A.Y. Yang, and B. Cukic. Fingerprint liveness detection based on histograms of invariant gradients. In Proc. IJCB, Clearwater, FL, USA, September 2014.

[59] C. Gottschlich and C.-B. Schönlieb. Oriented diffusion filtering for enhancing low-quality fingerprint images. IET Biometrics, 1(2):105-113, June 2012. 
[60] H. Fronthaler, K. Kollreider, and J. Bigun. Local features for enhancement and minutiae extraction in fingerprints. IEEE Transactions on Image Processing, 17(3):354-363, March 2008.

[61] D. Van De Ville, T. Blu, and M. Unser. Isotropic polyharmonic B-splines: scaling functions and wavelets. IEEE Transactions on Image Processing, 14(11):17981813, November 2005.

[62] L. Borup and M. Nielsen. Frame decomposition of decomposition spaces. Journal of Fourier Analysis and Applications, 13(1):39-70, February 2007.

[63] N. Otsu. A threshold selection method from gray-level histograms. IEEE Transactions on Systems, Man and Cybernetics, 9(1):62-66, January 1979.

[64] P. Sahoo, C. Wilkins, and J. Yeager. Threshold selection using Renyi's entropy. Pattern Recognition, 30(1):71-84, January 1997.

[65] M.P.d. Albuquerque, I.A. Esquef, A.R.G. Mello, and M.P.d. Albuquerque. Image thresholding using Tsallis entropy. Pattern Recognition Letters, 25(9):1059-1065, July 2004.

[66] T.F. Chan and L.A. Vese. Active contours without edges. IEEE Transactions on Image Processing, 10(2):266-277, February 2001.

[67] X. Bresson, S. Esedoglu, P. Vandergheynst, J.P. Thiran, and S. Osher. Fast global minimization of the active contour/snake model. Journal of Mathematical Imaging and Vision, 28(2):151-167, June 2007.

[68] T.F. Chan, S. Esedoglu, and M. Nikolova. Algorithms for finding global minimizers of image segmentation and denoising models. SIAM J. Appl. Math., 66(5):1632-1648, February 2012.

[69] J. Lie, M. Lysaker, and X.C. Tai. A binary level set model and some applications to Mumford-Shah image segmentation. IEEE Transactions on Image Processing, 15(5):1171-1181, May 2006.

[70] C. Sagiv, N.A. Sochen, and Y.Y. Zeevi. Integrated active contours for texture segmentation. IEEE Transactions on Image Processing, 15(6):1633-1646, June 2006 .

[71] N. Houhou, J.P. Thiran, and X. Bresson. Fast texture segmentation based on semi-local region descriptor and active contour. Numer. Math. Theor. Meth. Appl., 2(4):445-468, November 2009.

[72] L. Rudin, S. Osher, and E. Fatemi. Nonlinear total variation based noise removal algorithms. Physica D, 60(1-4):259-268, November 1992.

[73] J.F. Aujol, G. Gilboa, T. Chan, and S. Osher. Structure-texture image decomposition - modeling, algorithms, and parameter selection. International Journal of Computer Vision, 67(1):111-136, April 2006.

[74] J.F. Aujol, G. Aubert, L.B. Feraud, and A. Chambolle. Image decomposition into a bounded variation component and an oscillating component. Journal of Mathematical Imaging and Vision, 22(1):71-88, January 2005.

[75] J.F. Aujol and G. Gilboa. Constrained and SNR-based solutions for TV-Hilbert 
space image denoising. Journal of Mathematical Imaging and Vision, 26(1-2):217237, November 2006.

[76] A. Buades, T.M. Le, J. M. Morel, and L.A. Vese. Fast cartoon + texture image filters. IEEE Transactions on Image Processing, 19(8):1978-1986, August 2010.

[77] L.A. Vese and S. Osher. Modeling textures with total variation minimization and oscillatory patterns in image processing. Journal of Scientific Computing, 19(1-3):553-572, December 2003.

[78] G. Aubert and L. Vese. A variational method in image recovery. SIAM J. Numer. Anal, 34(5):1948-1979, October 1997.

[79] T. Chan, A. Marquina, and P. Mulet. High-order total variation-based image restoration. SIAM Journal on Scientific Computing, 22(2):503-516, July 2000.

[80] M. Lysaker, A. Lundervold, and X.C. Tai. Noise removal using fourth-order partial differential equation with applications to medical magnetic resonance images in space and time. IEEE Transactions on Image Processing, 12(12):1579-1590, December 2003.

[81] W.Zhu and T. Chan. Image denoising using mean curvature of image surface. SIAM Journal on Imaging Sciences, 5(1):1-32, January 2012.

[82] X.C. Tai, J. Hahn, and G.J. Chung. A fast algorithm for Euler's elastica model using augmented Lagrangian method. SIAM Journal on Imaging Sciences, 4(1):313-344, February 2011.

[83] G. Song, H. Zhang, and F.J. Hickernell. Reproducing kernel Banach spaces with the $\mathrm{L}_{1}$ norm. Applied and Computational Harmonic Analysis, 34(1):96-116, January 2013.

[84] W. Yin, S. Osher, D. Goldfarb, and J. Darbon. Bregman iterative algorithms for $\mathrm{L}_{1}$ minimization with applications to compressed sensing. SIAM Journal on Imaging Sciences, 1(1):143-168, March 2008.

[85] W. Yin, D. Goldfarb, and S. Osher. Image cartoon-texture decomposition and feature selection using the total variation regularization $\mathrm{L}_{1}$ functional. In Proc. $V L S M$, pages 73-84, Beijing, China, October 2005.

[86] J. Yang and Y. Zhang. Alternating direction algorithms for 11-problems in compressive sensing. SIAM Journal on Scientific Computing, 33(1):250-278, February 2011.

[87] E. Candès, M. Wakin, and S. Boyd. Enhancing sparsity by reweighted 11 minimization. J. Fourier Anal. Appl., 14(5):877-905, 2008.

[88] A. Chambolle. An algorithm for total variation minimization and applications. Journal of Mathematical Imaging and Vision, 20(1-2):89-97, January 2004.

[89] T. Goldstein and S. Osher. The split Bregman method for L1-regularized problems. SIAM Journal on Imaging Sciences, 2(2):323-343, April 2009.

[90] A. Beck and M. Teboulle. A fast iterative shrinkage-thresholding algorithm for linear inverse problems. SIAM Journal on Imaging Sciences, 2(1):183-202, January 2009 . 
[91] I. Daubechies, M. Defrise, and C. D. Mol. An iterative thresholding algorithm for linear inverse problems with a sparsity constraint. Communications on Pure and Applied Mathematics, 57(11):1413-1457, August 2004.

[92] J.B. Dias and M. Figueiredo. A new twist: Two-step iterative shrinkage/thresholding algorithms for image restoration. IEEE Transactions on Image Processing, 16(12):2992-3004, December 2007.

[93] C. Wu and X. C. Tai. Augmented Lagrangian method, dual methods, and split Bregman iteration for ROF, vectorial TV, and higher order methods. SIAM Journal on Imaging Sciences, 3(3):300-339, July 2010.

[94] A. Chambolle, R.A. DeVore, N.Y. Lee, and B.J. Lucier. Nonlinear wavelet image processing: variational problems, compression, and noise removal through wavelet shrinkage. IEEE Transactions on Image Processing, 7(3):319-335, March 1998.

[95] H. Choi and R.G. Baraniuk. Multiple wavelet basis image denoising using Besov ball projections. IEEE Signal Processing Letters, 11(9):717-720, September 2004.

[96] E. Candès and D. Donoho. New tight frames of curvelets and optimal representations of objects with piecewise singularities. Communications on Pure and Applied Mathematics, 57(2):219-266, February 2004.

[97] Starck, D.L. Donoho, and E.J. Candès. Astronomical image representation by the curvelet transform. Astron. Astrophys., 398(2):785-800, February 2003.

[98] M. Unser and D. Van De Ville. Wavelet steerability and the higher-order Riesz transform. IEEE Transactions on Image Processing, 19(3):636-652, March 2010.

[99] K. Frick, P. Marnitz, and A. Munk. Statistical multiresolution dantzig estimation in imaging: Fundamental concepts and algorithmic framework. Electronic Journal of Statistics, 6:231-268, 2012.

[100] K. Frick, P. Marnitz, and A. Munk. Statistical multiresolution estimation for variational imaging: With an application in poisson-biophotonics. Journal of Mathematical Imaging and Vision, 46(3):370-387, July 2013.

[101] J.F. Aujol and A. Chambolle. Dual norms and image decomposition models. International Journal of Computer Vision, 63(1):85-104, June 2005.

[102] J.B. Garnett, T.M. Le, Y. Meyer, and L.A. Vese. Image decompositions using bounded variation and generalized homogeneous Besov spaces. Applied and Computational Harmonic Analysis, 23(1):25-56, July 2007.

[103] W. Zhu, X.C. Tai, and T. Chan. Augmented Lagrangian method for a mean curvature based image denoising model. Inverse Problems and Imaging, 7(4):1409 - 1432, November 2013.

[104] A. Cohen, I. Daubechies, and J.C. Feauveau. Biorthogonal bases of compactly supported wavelets. Communications on Pure and Applied Mathematics, 45(5):485-560, June 1992.

[105] M.R. Leadbetter, G. Lindgren, and H. Rootzén. Extremes and Related Properties of Random Sequences and Processes. Springer, New York, 1983.

[106] T.F. Chan and S. Esedoglu. Aspects of total variation regularized $\mathrm{L}_{1}$ function 
approximation. SIAM Journal on Applied Mathematics, 65(5):1817-1837, May 2005 .

[107] J. De Boer, A. M. Bazen, and S. H. Gerez. Indexing fingerprint databases based on multiple features. In Proc. ProRisc Workshop on Circuits, Systems and Signal Processing, pages 300-306, 2001.

[108] O. Scherzer, editor. Handbook of Mathematical Methods in Imaging. Springer, New York, NY, USA, 2011.

[109] O. Scherzer, M. Grasmair, H. Grossauer, M. Haltmeier, and F. Lenzen. Variational Methods in Imaging. Springer, 2009. 


\section{Curriculum Vitae}

Duy Hoang Thai

born 16th February, 1985 in Ho Chi Minh city, Vietnam

Sep. 2003-Feb. 2008

- Degree: BSc. degree in the honor program of Electrical-Electronics Engineering from Ho Chi Minh city University of Technology, Vietnam.

- Thesis: Computer vision in controlling the movement of robot

- Supervisor: A.Prof. Dr. Nguyen Phuong Ha

Feb. 2008-Sep. 2009

Research Assistant in Automation lab, HCM University of Transport, Vietnam

Sep. 2009-August. 2011

- Degree: MSc in Bio-Mechatronics Engineering, Sungkyunkwan University, Korea.

Research Assistant in Robotics and Vision lab

- Thesis: Human Iris Recognition

- Supervisor: Prof. Dr. Heon Hwang

Sep. 2011-Jan.2015

- Degree: Phd in Computer Science, University of Goettingen, Germany member of the DFG Graduate Program 1023

"Identification in Mathematical Models"

- Thesis: Fourier and Variational Based Approaches for Fingerprint Segmentation

- Supervisor: Prof. Dr. Axel Munk and Prof. Dr. Preda Mihăilescu 